\title{
CATÁLOGO DOS EROTYLIDAE (COLEOPTERA) NEOTROPICAIS
}

\author{
Moacyr Alvarenga ${ }^{1}$
}

\begin{abstract}
Catalogue of Neotropical Erotylidae (Coleoptera). The present catalog is an inventory of names proposed in Erotylidae (from family through subspecies) ocurring in the Neotropical Region, up to the present date (1988). It updates the nomenclature of included taxa, adds taxonomic units proposed after the Kuhnt's Genera Insectorum (KUHNT, 1909) and includes abbreviated references to the literature, which are fully cited at the end of this catalogue. The systematic order follows KUHNT, 1909, and afterwards, the position given to each taxa by the original author.
\end{abstract}

\section{CONTEÚDO}

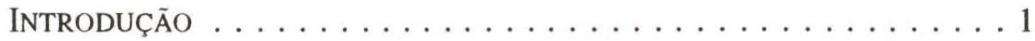

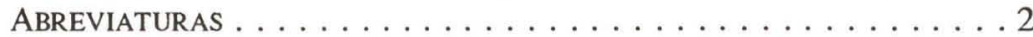

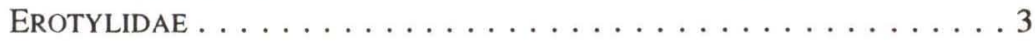

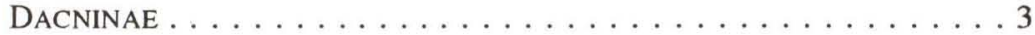

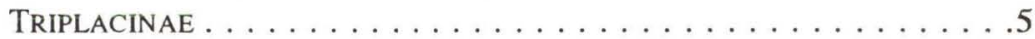

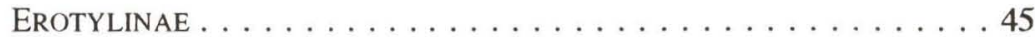

REFERÊNCIAS BIBLIOGRÁFICAS . . . . . . . . . . . . . . 125

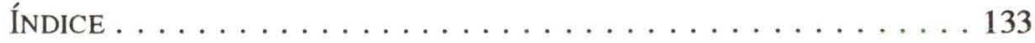

O presente trabalho é um inventário dos nomes propostos em Erotylidae (de família a subespécie) de ocorrência neotropical, até a presente data (1988).

Atualiza a nomenclatura, adiciona unidades taxonômicas após a publicação do Genera Insectorum (KUHNT 1909) e fornece indicações bibliográficas resumidas, cuja citação integral aparece no fim do catálogo.

A ordem sistemática utilizada foi a do Genera Insectorum (KUHNT 1909) e posteriormente a esta data, adotou-se a posição dada a cada táxon pelo próprio autor.

A cada espécie arrolada consta a continuação do nome específico, do autor, do ano e página de publicação; entre parênteses, o gênero em que foi originalmente descrita, a instituição onde o material-tipo encontra-se depositado (quando o mesmo foi localizado, caso contrário, aparece um ponto de interrogação); a localidade-tipo, entre parênteses, as referências e, ao final da listagem, a distribuição geográfica por país - no caso do Brasil, por regiões.

1) Pesquisador Associado, Departamento de Entomologia, Museu Nacional do Rio de Janeiro, Quinta da Boa Vista, 20940-040 Rio de Janeiro, Rio de Janeiro, Brasil. 


\section{ABREVIATURAS}

ab. - aberração

AMNH - American Museum of Natural History, New York, USA

ANSP - Academy of Natural Sciences of Philadelphia, USA

CARJ - Coleção Alvarenga, Rio de Janeiro

CAS - California Academy of Sciences, San Francisco, USA

CDSP - Coleção Diringshofen (atualmente incorporada ao Museu de ZoologiaUSP), São Paulo

DEIB - Deutsches Entomologisches Institut, Berlin, Alemanha

DEME - Deutsches Entomologisches Museum, Eberswalde, Alemanha

desig. orig. - designação original

Distr. - Distribuição

DTNY - Department of Tropical Research, New York Zoological Park, USA exam. aut. - examinado pelo autor

FIOC - Fundação Instituto Oswaldo Cruz, Rio de Janeiro

FMNH - Field Museum of Natural History, Chicago, USA

HCGU - Hunterian Collection, Glasgow University, Inglaterra

IMLT - Facultad de Ciencias Naturales e Instituto Miguel Lillo, Tucumán, Argentina

MCZC - Museum of Comparative Zoology, Cambridge, Massachusetts, USA

MGFM - Museums Georg Frey,Tutzing bei München, Alemanha

MHMW - Naturhistorisches Museum, Wien, Áustria

MLPA - Museu de La Plata, La Plata, Argentina

MNHN - Muséum National d'Histoire Naturelle, Paris, França

MNRJ - Museu Nacional, Rio de Janeiro

comb.n. - nova combinação

nom.praeoc. - nome ocupado

NHML - The Natural History Museum, London, Inglaterra

NKMS - Naturkundemuseum, Stettin, Alemanha

NMNH - National Museum of Natural History, Washington, D.C., USA

PULI - Purdue University, Lafayette, Indiana, USA

RNHL - Rijmuseum van Natuurlijke Historie, Leiden, Alemanha

seg. - segundo

syn. - sinônimo

SMTD - Staatliches Museum für Tierkunde, Dresden, Alemanha

Tgen. - espécie-tipo do gênero

Tp. - tipo 
Tsubgen. - espécie-tipo do subgênero

UFPR - Universidade Federal do Paraná, Curitiba

UK - Kansas State University, Manhattan, USA

ZMCD - Zoological Museum of Copenhague, Dinamarca

ZMHU - Zoologisches Museum, Humboldt Universität, Berlin, Alemanha

ZMUH - Zoologisches Museum, Universität Hamburg, Alemanha

ZSBS - Zoologische Staatssammlung, München, Alemanha

\section{EROTYLIDAE Duponchel, 1825}

Erotylidae Duponchel, 1825:30-61, 156-176, pls. 1, 2 e 7, 86 figs (Erotylites). Lacordaire, 1842:1-543 (Erotyliens). - Chapuis, 1876:1-72. - Crotch, 1876: 396-572 (Erotylidae). - Gemminger \& Harold, 1876:3681-3721. - Fleutiaux, 1886: 253-257. - Gorham, 1887-1889:33-114, pls. 2-6, 100 figs; 1898-1899: 253-257. - Kuhnt, 1909:1-139, pls. 1-4, 65 figs; 1911:1-103. - Bruch, 1914:346-400. - Leng, 1920:200-201. - Deelder, 1942:49-115, 35 figs Blackwelder, 1945:456-468. - Boyle, 1956:61-172, 141 figs - Alvarenga, 1965:75-92. - Delkeskamp, 1981:9. - Chûjô \& Chûjô, 1988:139.

\section{DACNINAE Crotch, 1876}

Lacordaire, 1842:33-73 (Erotyliens engidiformes). - Chapuis, 1876:19-30 (Engidites). - Crotch, 1876:396-418 (Dacnini). - Kuhnt, 1909:93-116. - Gorham, 1887:33-34 (Dacnides). - Boyle, 1956:78-93 (Dacninae). - Delkeskamp, 1981:10. - Chûjô \& Chûjô, 1988:140.

\section{Dacne Latreille, 1796}

Dacne Latreille, 1796:12 (Tgen.: Dermestes bipustulatus Thunberg, 1781:6, seg. Latreille, 1810:427). - Lacordaire, 1842:63. - Crotch, 1873a:352; 1876:396.

- Chapuis, 1876:21. - Kuhnt, 1909:94, 96. - Casey, 1916:152. - Deelder, 1942:54. - Boyle, 1956:78,81. - Delkeskamp, 1981:10. - Chûjô \& Chûjô, 1988: 141 .

Engis Paykull, 1800:349 (Tgen.: Ips humeralis Fabricius, 1787). - Lacordaire, 1842:63. - Chûjô \& Chûjô, 1988:141. (syn.).

californica Horn, 1870:97 (Engis). Tp.: ANSP - (California). - Crotch, 1873a:352 (Dacne); 1876:397. - Kuhnt, 1909:98, pl. 4, fig. 1; 1911:69. - Casey, 1916:154 - Boyle, 1956:82-83, figs 5, 19, 20 e 54. Distr.: Canadá e USA, região oeste. México, Baja California. 


\section{Megalodacne Crotch, 1873}

Megalodacne Crotch, 1873a:352 (Tgen.: Ips fasciata Fabricius, 1776:213, desig. orig.). - Gorham, 1887:33. - Kuhnt, 1909:114. - Heller, 1918a:141, 156. Deelder, 1942:56. - Delkeskamp, 1952:62. - Boyle, 1956:78, 88. - Alvarenga, 1965:86. - Delkeskamp, 1981:11. - Chûjô \& Chûjô, 1988:177.

\section{Megalodacne (Psephodacne) Heller, 1918}

Psephodacne Heller, 1918a:141 (Tsubgen.: Silpha indica Linnaeus, 1758:360, desig. orig.); 1920:57. - Delkeskamp, 1952:65, 71, 73. - Alvarenga, 1965:88. - Delkeskamp, 1981:21.

Romodacne Delkeskamp, 1937:49 (Tsubgen.:Ips grandis Fabricius, 1792:511, desig. orig.); 1952:65. - Delkeskamp, 1981:21 (Romodacne) (syn.).

audouini Lacordaire, 1842:66 (Dacne). Tp. ? - (México).- Crotch, 1876:416 (Megalodacne). Gemminger \& Harold, 1876, :3682. - Gorham, 1887:34, pl. 2, fig. 2; 1898:253-Kuhnt, 1909:115; 1911:80. - Deelder, 1942:112. -Blackwelder, 1945:468. - Delkeskamp, 1952:70, 72, fig. 5b. Distr.: México.

ab. unifasciata Schenkling, 1919:93. Tp.: DEIB exam. aut. - (México). Deelder, 1942:112. - Blackwelder, 1945:468. - Delkeskamp, 1952:72. Distr.: México.

batesi Crotch, 1876:416. Tp. : MCZC exam. aut. - (Brasil, Amazonas). Gemminger \& Harold, 1876:3682. - Kuhnt, 1909:115; 1911:80. - Blackwelder, 1945:468. - Delkeskamp, 1952:70, 72, 73, figs 4, 5a, 6, 7. Distr.: Brasil, região amazônica.

indica indica Linnaeus, 1758:360 (Silpha). Tp. ? - (América meridional). Fabricius, 1781:85. - Bedel, 1919:114 (Megalodacne). - Mader, 1942d: 176; 1951:225. - Delkeskamp, 1952:67, 72. Distr.: Da América Central até a Argentina.

signata Laporte, 1840:15 (Engis). - Lacordaire, 1842:70 (syn.).

heros; Guérin-Méneville, 1841, nec Say 1823. - Guérin-Méneville, 1841:159 (Episcapha). - Lacordaire, 1842:70 (nom.praeoc.).

quadriguttata Olivier, 1792:434 (Erotylus); 1807:489, pl. 1, fig.2(Triplax). Lacordaire, 1842:70 (Dacne). - Crotch, 1873b:141 (Megalodacne); 1876:416. - Gemminger \& Harold, 1876:3683. - Gorham, 1887:34, pl. 2, fig. 1. - Kuhnt, 1909:114, pl.4, fig. 8; 1911:80. - Bruch, 1914:385.

- Deelder, 1942:112. - Guérin, 1948:21. - Bedel, 1919:114 (syn.).

indica latifasciata Delkeskamp, 1952:69, 72, 73, fig. 3. Tp.: ZMHU - (Peru, Cumbare, Tarapoto). Distr.: Peru, Equador.

indica multifida Lacordaire, 1842:72 (Dacne). Tp. ? - (Brasil). - Crotch, 1876:416 (Megalodacne). - Kuhnt, 1909:115; 1911:80. - Guérin, 1948:21. - Delkes- 
kamp, 1952:68, 72, 73, figs 8-11 (comb.n.). Distr.: Brasil, região sudeste. brasiliensis Lacordaire, 1842:72 (Dacne). - Crotch, 1876:416 (Megalodacne). - Kuhnt, 1909:115; 1911:80. - Guérin, 1948:21. - Delkeskamp, 1952: 68, 73 (syn.).

tortuosa Lacordaire, 1842:69 (Dacne). Tp. ? - (México). - Crotch, 1876:416 (Megalodacne). - Gemminger \& Harold, 1876:3683. - Gorham, 1887:34. - Kuhnt, 1909:115; 1911:81. - Deelder, 1942:112. - Blackwelder, 1945: 468. - Delkeskamp, 1952:70, 72, 73. Distr.: México. Colômbia.

\section{TRIPLACINAE Crotch, 1876}

Chapuis, 1876:30-48 (Triplacites). - Crotch, 1876:418-481 (Triplacini). - Kuhnt, 1909:54-93. - Gorham, 1888:35-82 (Triplacides). - Boyle, 1956:78, 96-141 (Triplacinae).

\section{Pselaphacus Percheron, 1835}

Pselaphacus Percheron, 1835, 4e. livre, $\mathrm{n}^{\circ}$ 6. (Tgen.: Pselaphacus nigropunctatus Percheron, 1835. seg. Crotch, 1873b:141). - Lacordaire, 1842:30, 73. Crotch, 1873b:141; 1876:418. - Chapuis, 1876:31. - Gorham, 1887:35. Kuhnt, 1909:58. - Deelder, 1942:52. - Alvarenga, 1965:88.

amazonicus Crotch, 1876:421. Tp.: MCZC exam. aut. - (Brasil, Amazonas). Gemminger \& Harold, 1876:3688. - Kuhnt, 1909:59; 1911:39. - Blackwelder, 1945:464. Distr.: Brasil, região amazônica.

approximatus Crotch, 1876:422. Tp.: MCZC exam. aut. - (Brasil, Amazonas). Gemminger \& Harold, 1876:3688. - Kuhnt, 1909:59; 1911:39. - Blackwelder, 1945:464. Distr.: Brasil, região amazônica.

atricollis Kuhnt, 1910:226. Tp.: MLPA - (Argentina, Missões); 1911:39. - Bruch, 1914:382. - Blackwelder, 1945:464. Distr.: Argentina.

concinnus Kuhnt, 1910:227. Tp. ? - (Guatemala); 1911:39. - Blackwelder, 1945:464. Distr.: Guatemala.

confusus Crotch, 1876:420. Tp.: MCZC exam. aut. - (Brasil, Pará). - Gemminger \& Harold, 1876:3688. - Kuhnt, 1909:59; 1911:39. - Blackwelder, 1945: 464. Distr.: Brasil, região amazônica.

conspersus Kirsch, 1883:211. Tp.: SMTD exam. aut. - (Colômbia, Bogotá).

Fleutiaux, 1886:222. - Kuhnt, 1909:58; 1911:39. - Blackwelder, 1945:464.

Distr.: Colômbia.

contaminatus Erichson, 1847:180. Tp.: ZMHU - (Peru). - Crotch, 1876:419. Gemminger \& Harold, 1876:3688. - Kuhnt, 1909:58; 1911:39. - Mader, 1942d:169; 1951:206. - Blackwelder, 1945:464. Distr.: Peru.

curvipes Guérin-Méneville, 1841b:157. Tp.: MCZC ex.aut. - (Bolívia). - Lacor- 
daire, 1842:81. - Crotch, 1876:420. - Gemminger \& Harold, 1876:3688. - Gorham, 1887:35, pl. 2, fig. 5, 6. - Kuhnt, 1909:59; 1911:39. - Deelder, 1942:81. - Mader, 1942d:170; 1951:206. - Blackwelder, 1945:464. Guérin, 1948:19. Distr.: Do México até o sul do Brasil.

gracilipes Lacordaire, 1842:82. - Crotch, 1876:420 (syn.).

distortus Crotch, 1873b:142. - Gorham, 1887:35 (syn.).

dentatus Germar, 1824:615 (Triplax). Tp.: ZMHU exam. aut. - (Brasil).

Lacordaire, 1842:87 (Pselaphacus). - Crotch, 1876:421. - Gemminger \& Harold, 1876:3688. - Kuhnt, 1909:59; 1911:39. - Blackwelder, 1945:464.

- Guérin, 1948:19. Distr.: Brasil, regiões sudeste e sul.

ducalis Crotch, 1876:419. Tp.: MCZC exam. aut. - (Colômbia). Gemminger \& Harold, 1876:3688. - Kuhnt, 1909:59; 1911:39. - Blackwelder, 1945:464. Distr.: Colômbia.

giganteus Germar, 1824:615 (Triplax). Tp.: ZMHU exam. aut. - (América meridional). - Lacordaire, 1842:76 (Pselaphacus). - Crotch, 1876:419. Gemminger \& Harold, 1876:3688. - Kuhnt, 1909:59; 1911:39. - Curran, 1941:287. - Deelder, 1942:81. - Blackwelder, 1945:464. Distr.: Guiana. Suriname. Guiana Francesa.

cayennensis Laporte, 1840:15 (Engis). - Lacordaire, 1842:76 (syn.).

gorhami Kuhnt, 1909:59; 1911:39. - Deelder, 1942:81. - Blackwelder, 1945:464.

Distr.: Panamá.

conspersus Gorham, 1887 nec Kirsch, 1883. - Gorham, 1887:35, pl. 2, fig. 10. Tp.: NHML - (Panamá, Volcán de Chiriquí). - Kuhnt, 1909:59 (nom.praeoc.).

maculatus Guérin-Méneville, 1841b:158, Tp.: MNHN exam. aut. - (Bolívia). Lacordaire, 1842:79. - Crotch, 1876:419. - Gemminger \& Harold, 1876: 3688. - Kuhnt, 1909:59; 1911:39. - Blackwelder, 1945:464. - Delkeskamp, 1957:98. Distr.: Bolívia.

meandrinus Lacordaire, 1842:86. Tp. ? - (Colômbia). - Crotch, 1876:421.

Gemminger \& Harold, 1876:3688. - Kuhnt, 1909:59; 1911:39. - Blackwelder, 1945:464. Distr.: Colômbia. Equador.

mysticus Gorham, 1883:84. Tp.: NHML exam. aut. - (Peru, Chanchamayo).

Fleutiaux, 1886:222. - Kuhnt, 1909:59; 1911:39. - Mader, 1942d:169;

1951:206. - Blackwelder, 1945:464. Distr.: Peru.

nicaraguae Crotch, 1873b: 142. Tp.: NHML exam. aut. - (Nicarágua, Chontales, Santo Domingo); 1876:422. - Gemminger \& Harold, 1876:3688. Fleutiaux, 1886:222. - Gorham, 1887:36, pl. 2, fig. 3. - Kuhnt, 1909:59; 1911:39. - Blackwelder, 1945:464. Distr.: Nicarágua. Panamá.

nigropictus Crotch, 1876:420. Tp.: MCZC exam. aut. - (Brasil, Amazonas).

Gemminger \& Harold, 1876:3688. - Kuhnt, 1909:59; 1911:39. - Blackwelder, 1945:464. Disir.: Brasil, região amazônica.

nigropunctatus Percheron, 1835, 4e. livre, $\mathrm{n}^{\circ}$ 6, pl. 17. Tp: MNHN exam. aut. 
[Localidade não citada] - Lacordaire, 1842:75. - Crotch, 1876:418. Gemminger \& Harolt, 1876:3688. - Kuhnt, 1909:58; 1911:39. - Bruch, 1914:382. - Deelder, 1942:81.- Mader, 1942d:169; 1951:205. - Blackwelder, 1945:464. - Delkeskamp, 1957:98. Distr.: Equador. Peru. Bolívia. Brasil, região amazônica. Argentina.

poecilosomus Lacordaire, 1842:77. Tp.: MCZC exam. aut. - (Colômbia). Crotch, 1876:419. - Gemminger \& Harold, 1876:3688. - Gorham, 1887: 35, pl. 2, fig. 4. - Kuhnt, 1909:59; 1911:39. - Deelder, 1942:81. Blackwelder, 1945:464. Distr.: Panamá. Colômbia. Equador.

hopei Guérin-Méneville, 1844:309. - Crotch, 1876:419 (syn.).

procerus Kuhnt, 1910:226. Tp.: ZMHU exam. aut. - (Argentina, Missões); 1911:40. - Bruch, 1914:382. - Blackwelder, 1945:464. Distr.: Argentina.

punctatostriatus Crotch, 1876:420. Tp.: MCZC exam. aut. - (Brasil, Pará). Gemminger \& Harold, 1876:3688. - Kuhnt, 1909:59; 1911:40. - Blackwelder, 1945:464. Distr.:Brasil, região amazônica.

puncticollis Guérin-Méneville, 1841 b:158. Tp.: MCZC exam. aut. - (Colômbia); 1855:610. - Lacordaire, 1842:87; 1876, pl. 131, fig. 5. - Crotch, 1876:421. - Gemminger \& Harold, 1876:3688. - Gorham, 1887:36, pl. 2, fig. 8. Kuhnt, 1909:59; 1911:40. - Bruch, 1914:382. - Deelder, 1942:81 - Mader, 1942d:170; 1951:206. - Blackwelder, 1945:464. - Guérin, 1948:19. Delkeskamp, 1957:98. Distr.: Do México até Argentina.

quinquenotatus Lacordaire, 1842:78. Tp.: MHNP exam. aut. -(Caiena). - Crotch, 1876:419. - Gemminger \& Harold, 1876:3688. - Kuhnt, 1909:59; 1911:40.

- Deelder, 1942:81. - Blackwelder, 1945:464. Distr.: Guiana Francesa. Brasil, região amazônica.

rubricatus Herbst, 1799:363, pl. 137, fig. 2 (Erotylus). Tp.: ZMHU exam. aut. - [Localidade não citada]. - Schönher, 1808:328. - Lacordaire, 1842:85 (Pselaphacus). - Crotch, 1876:422. - Gemminger \& Harold, 1876:3688. Kuhnt, 1909:59; 1910:227; 1911:40. - Deelder, 1942:81. - Mader, 1942d: 170; 1951:206. - Blackwelder, 1945:464. Distr.: Suriname. Guiana Francesa. Equador. Peru. Brasil, região amazônica.

catenulatus Olivier, 1807:489, pl. 1, fig. 1a, 1b. (Triplax). - Lacordaire, 1842:85 (syn.).

semiclathratus Lacordaire, 1842:88. Tp.: MCZC exam. aut. - (México, Yucatán). - Crotch, 1876:421. - Gemminger \& Harold, 1876:3688. - Gorham, 1887:36, pl. 2, fig. 9. - Kuhnt, 1909:59; 1911:40. - Blackwelder, 1945: 464. Distr.: América Central.

signatus Guérin-Méneville, 1841b;158. Tp.: MNHN exam. aut. - (Bolívia). Crotch, 1873b:142; 1876:421. - Gemminger \& Harold, 1876:3688. Gorham, 1887:37. - Kuhnt, 1909:59; 1911:40. - Bruch, 1914:382. Curran, 1941:287. - Deelder, 1942:82. - Mader, 1942d:170; 1951:206. Blackwelder, 1945:464. Distr.: Da América Central até a Argentina. 
signatipennis Lacordaire, 1842:84. - Crotch, 1873b: 142 (syn.).

sparsus Lacordaire, 1842:80. Tp.: MCZC exam. aut. - (Brasil, Pará). - Crotch,

1876:419. - Gemminger \& Harold, 1876:3688. - Kuhnt, 1909:58; 1911:40.

- Blackwelder, 1945:464. Distr.: Brasil, região amazônica.

var. paraensis Guérin, 1956:61. Tp.: UFPR exam. aut. - (Brasil, Pará, Mocajuba, Mangabeira). Distr.: Brasil, região amazônica.

transversalis Lacordaire, 1842:77. Tp. ? - (Caiena). - Guérin-Méneville, 1855:

610. - Crotch, 1876:419. - Gemminger \& Harold, 1876:3689. - Kuhnt, 1909:58; 1911:40. - Blackwelder, 1945:464. Distr.: Guiana Francesa.

trifasciatus Lacordaire, 1842:83. Tp. ? - (Caiena). - Crotch, 1876:420. - Gemminger \& Harold, 1876:3689. - Kuhnt, 1909:59, pl. 3, fig. 3, 3a; 1911:40.

- Deelder, 1942:82. - Blackwelder, 1945:464. Distr.: Guiana Francesa.

Bolívia. Brasil, região amazônica.

vitticollis Crotch, 1873b:142. Tp.: NHML exam. aut. - (Colômbia); 1876:421. Gemminger \& Harold, 1876:3689. - Gorham, 1887:36, pl. 2, fig. 7. Kuhnt, 1909:59; 1911:40. - Blackwelder, 1945:464. Distr.: Nicarágua. Panamá. Colômbia.

\section{Ischyrus Chevrolat, 1837}

Ischyrus Chevrolat, 1837:428 (Tgen.: Erotylus undatus Olivier, 1792:434, seg. Alvarenga, 1965:85). - Lacordaire, 1842:31, 89. - Chapuis, 1876:31, 35. Alvarenga, 1965:85.

Megischyrus Crotch, 1873b:143 (Tgen.: Erotylus undatus Olivier, 1792:434, desig. orig.). - Gorham, 1887:37. - Kuhnt, 1909:57, 60. - Deelder, 1942:53. - Guérin, 1952:180. - Alvarenga, 1965:86 (syn.).

angustatus Lacordaire, 1842:96. Tp. ? - (Colômbia). - Crotch, 1876:423 (Megischyrus). - Gemminger \& Harold, 1876:3689. - Kuhnt, 1909:61; 1911:40. - Blackwelder, 1945:464. Distr.: Colômbia.

bartleti Gorham, 1883:85, pl. 18, fig. 9 (Megischyrus). Tp.: NHML exam. aut. - (Peru, Chamicuros). - Fleutiaux, 1886:222. - Kuhnt, 1909:61; 1911:40. - Mader, 1942d:170; 1951:207. - Blackwelder, 1945:464. Distr.: Peru.

bellicosus Lacordaire, 1842:98. Tp. ? - (Brasil). - Crotch, 1876:424 (Megischyrus). - Gemminger \& Harold, 1876:3689. - Kuhnt, 1909:61; 1911:40. - Schenkling, 1919:85. - Deelder, 1942:82. - Blackwelder, 1945:464. Guérin, 1948:19. Distr.: Brasil, região sudeste.

bifasciatus Guérin, 1952:180, fig. 2 (Megischyrus). Tp. ? - (Chile, Valdivia, Pemehue). Distr.: Chile.

bogotae Crotch, 1876:424 (Megischyrus). Tp.: MCZC exam. aut. - (Colômbia, Bogotá). - Gemminger \& Harold, 1876:3689. - Kuhnt, 1909:61; 1911:40. - Blackwelder, 1945:464. Distr.: Colômbia. 
brasiliensis Lacordaire, 1842:91. Tp. ? - (Brasil). - Crotch, 1876:422 (Megischyrus). - Gemminger \& Harold, 1876:3690. - Kuhnt, 1909:60; pl. 3, fig. 5, 1911:40. - Bruch, 1914:382. - Deelder, 1942:82. - Blackwelder, 1945:464. - Guérin, 1948:19. Distr.: Colômbia. Suriname. Brasil. Argentina.

catenatus Crotch, 1876:424 (Megischyrus). Tp.: MCZC exam. aut. - (Brasil, Amazonas). - Gemminger \& Harold, 1876:3689. - Kuhnt, 1909:61; 1911: 40. - Curran, 1941:287. - Mader, 1942d:170; 1951:208. - Blackwelder, 1945:464. Distr.: Colômbia. Guiana. Equador. Peru. Brasil, região amazônica.

catenulatus Lacordaire, 1842:97. Tp. ? - (Bolívia, Santa Cruz de la Sierra). Crotch, 1876:424 (Megischyrus). - Gemminger \& Harold, 1876:3689. Kuhnt, 1909:61; 1911:40. - Blackwelder, 1945:464. Distr.: Bolívia.

chevrolati Crotch, 1876:423 (Megischyrus). Tp.: MCZC exam. aut. - (Brasil, Minas Gerais). - Gemminger \& Harold, 1876:3689. - Kuhnt, 1909:60; 1911:41. - Blackwelder, 1945:464. - Guérin, 1948:19. Distr.: Brasil, região sudeste.

circumscriptus Duponchel, 1825:165, pl. 7, fig. 83 (Erotylus). Tp. ? - (Brasil). Lacordaire, 1842:102 (Ischyrus). - Crotch, 1876:425 (Megischyrus). Gemminger \& Harold, 1876:3689. - Kuhnt, 1909:60; 1911:41. - Blackwelder, 1945:464. Distr.: Brasil, região sudeste.

columbianus Lacordaire, 1842:95. Tp.: MCZC exam. aut. - (Colômbia). - Crotch, 1876:423 (Megischyrus). - Gemminger \& Harold, 1876:3689. - Kuhnt, 1909:61; 1911:41. - Deelder, 1942:82. - Mader, 1942d:170; 1951:207. Blackwelder, 1945:464. Distr.: Colômbia. Venezuela. Peru.

connexus Crotch, 1876:423 (Megischyrus). Tp.: MCZC exam. aut. - (Colômbia). - Gemminger \& Harold, 1876:3689. - Kuhnt, 1909:61; 1911:41. - Blackwelder, 1945:464. Distr.: Colômbia. Equador.

decempunctatus Guérin-Méneville, 1841b:154 Tp.: MNHN exam. aut. - (Bolívia). - Lacordaire, 1842:99. - Crotch, 1876:425 (Megischyrus). - Gemminger \& Harold, 1876:3689. - Kuhnt, 1909:61; 1911:41. - Deelder, 1942:82. - Blackwelder, 1945:464. - Delkeskamp, 1957:98. Distr.: Peru. Bolívia. Brasil, região sul.

discipennis Lacordaire, 1842:101. Tp. ? - (México). - Crotch, 1873b:143 (Megischyrus); 1876:425. - Gemminger \& Harold, 1876:3689. - Gorham, 1887: 39, pl. 2, fig. 15. - Kuhnt, 1909:60; 1911:41. - Deelder, 1942:82. Blackwelder, 1945:464. Distr.: México. Nicarágua. Panamá.

elongatus Gorham, 1883:85 (Megischyrus). Tp.: NHML exam. aut. - (Peru, Chanchamayo). - Fleutiaux, 1886:222. - Kuhnt, 1909:61; 1911:41. Mader, 1942d:170; 1951:208. - Blackwelder, 1945:464. Distr.: Peru.

var. gorhami Mader, 1942d:171 (Megischyrus). Tp. ? - (Peru); 1951:208. Distr.: Peru. 
guatemalae Crotch, 1876:424 (Megischyrus). Tp.: MCZC exam. aut. - (Guatemala). - Gemminger \& Harold, 1876:3689. - Gorham, 1887:38, pl. 2, fig. 12. - Kuhnt, 1909:61; 1911:41. - Blackwelder, 1945:464. Distr.: Belize. Guatemala.

jurinei Lacordaire, 1842:94. Tp. ? - (Bolívia, Santa Cruz de la Sierra). - Crotch, 1876:422 (Megischyrus). - Gemminger \& Harold, 1876:3689. - Kuhnt, 1909:60; 1910:228; 1911:41. - Mader, 1942d:170; 1951:208. - Blackwelder, 1945:464. - Delkeskamp, 1957:98. Distr.: Colômbia. Peru. Bolívia. Brasil, região amazônica.

knochi Lacordaire, 1842:100. Tp.: MNHN exam. aut. - (Bolívia, Santa Cruz de la Sierra). - Crotch, 1876:425 (Megischyrus). - Gemminger \& Harold, 1876:3689. - Kuhnt, 1909:60; 1911:41. - Blackwelder, 1945:464. Distr.: Bolívia.

laetus Schenkling, 1919:85 (Megischyrus). Tp.: DEIB exam. aut. - (Peru). Mader, 1942d:171; 1951:209. - Blackwelder, 1945:464. Distr.: Peru.

lineatus Lacordaire, 1842:104. Tp. ? - (Brasil, Rio de Janeiro). - Crotch, 1876:425 (Megischyrus). - Gemminger \& Harold, 1876:3689. - Kuhnt, 1909:60; 1911:41. - Blackwelder, 1945:464. Distr.: Brasil, região sudeste.

grammistes Lacordaire, 1842:105. - Crotch, 1876:425 (syn.).

mesomelas Crotch, 1876:423 (Megischyrus). Tp.: MCZC exam. aut. - (Equador). - Gemminger \& Harold, 1876:3689. - Kuhnt, 1909:61; 1911:41. - Blackwelder, 1945:464. Distr.: Equador.

mexicanus Lacordaire, 1842:93. Tp.: MCZC exam. aut. - (México, Orizaba). Crotch, 1876:423 (Megischyrus). - Gemminger \& Harold, 1876:3689. Gorham, 1887:37, pl. 2, fig. 11. - Kuhnt, 1909:61; 1911:41. - Deelder, 1942:82. - Blackwelder, 1945:464. Distr.: México.

var. consanguineus Lacordaire, 1842:94. Tp. ? - (México Yucatán). - Gemminger \& Harold, 1876:3689 (Megischyrus). - Kuhnt, 1909:61; 1911: 41. - Blackwelder, 1945:464. Distr.: México.

nicaraguae Crotch, 1873b:143 (Megischyrus). Tp.: NHML exam. aut. - (Nicarágua, Chontales, Santo Domingo); 1876:423. - Gemminger \& Harold, 1876:3689. - Gorham, 1887:37, pl. 2, fig. 13; 1898:253. - Kuhnt, 1909:61; 1911:41. - Deelder, 1942:82. - Blackwelder, 1945:464. Distr.: América Central.

var. panamae Schenkling, 1919:85 (Megischyrus). Tp.: DEIB exam. aut. (Panamá). - Blackwelder, 1945:464. Distr.: Panamá.

octostriatus Kuhnt, 1910:227, fig. 4 (Megischyrus). Tp.: ZMHU exam. aut. (Brasil); 1911:41. - Blackwelder, 1945:464. Distr.: Brasil, região sudeste. ohausi Mader, 1943:115 (Megischyrus). Tp.: MGFM exam. aut. - (Equador, Sabanilla). Distr.: Equador.

panamanus Mader, 1942c:571 (Megischyrus). Tp.: MGFM exam. aut. - (Panamá, Chiriquí). Distr.: Panamá. 
pictipennis Schenkling, 1919:84 (Megischyrus). Tp.: DEIB exam. aut. - (Venezuela). - Blackwelder, 1945:464. Distr.: Venezuela.

planior Kirsch, 1876:99. Tp.: SMTD exam. aut. - (Peru, Sarayacu). - Gemminger \& Harold, 1876:3689 (Megischyrus). - Kuhnt, 1909:60; 1911:41. - Schenkling, 1919:84. - Mader, 1942d:170; 1951:208. - Blackwelder, 1945:464. Distr.: Peru.

sanguinolentus Lacordaire, 1842:97. Tp. ? - (México, Yucatán). - Crotch, 1876:424 (Megischyrus). - Gemminger \& Harold, 1876:3689. - Gorham, 1887:38, pl. 2, fig. 14. - Kuhnt, 1909:61; 1911:41. - Deelder, 1942:82. Blackwelder, 1945:464. Distr.: México. América Central.

scaphinotus Lacordaire, 1842:96. Tp. ? - (Colômbia). - Crotch, 1876:423 (Megischyrus). - Gemminger \& Harold, 1876:3689. - Kuhnt, 1909:61; 1911:41. - Blackwelder, 1945:464. Distr.: Colômbia.

semipunctatus Germar, 1824:612 (Erotylus). Tp. ? - (Brasil). - Lacordaire, 1842:99 (Ischyrus). - Crotch, 1876:424 (Megischyrus). - Gemminger \& Harold, 1876:3689. - Kuhnt, 1909:61; 1911:41. - Deelder, 1942:82. Blackwelder, 1945:464. - Guérin, 1948:19. Distr.: Brasil, região sudeste. balteatus Duponchel, 1825:164, pl. 7, fig. 79 (Erotylus). - Lacordaire, 1842:99 (syn.).

semitinctus Erichson, 1847:180. Tp.: ZMHU exam. aut. - (Peru). - Crotch, 1876:425 (Megischyrus). - Gemminger \& Harold, 1876:3689. - Kuhnt, 1909:61; 1911:41. - Schenkling, 1919:85. - Deelder, 1942:82. - Mader, 1942d:170; 1951:208. - Blackwelder, 1945:464. Distr.: Peru.

sicarius Lacordaire, 1842:101. Tp. ? - (Brasil). - Crotch, 1876:425 (Megischyrus). - Gemminger \& Harold, 1876:3689. - Kuhnt, 1909:60; 1911:41. - Deelder, 1942:82. - Mader, 1942d:170; 1951:207. - Blackwelder, 1945:464. Distr.: Colômbia. Peru. Brasil, região amazônica.

var. perizonatus Lacordaire, 1842:103. Tp. ? -(Colômbia). - Crotch, 1876:425 (Megischyrus). - Gemminger \& Harold, 1876:3689. - Kuhnt, 1909:60; 1911:41. - Mader, 1942d:170; 1951:207. - Blackwelder, 1945:464. Distr.: Colômbia. Peru.

undatus Olivier, 1792:434 (Erotylus). Tp. ? - (Caiena); 1807:490, pl. 1, fig. 3 (Triplax). - Crotch, 1876:422 (Megischyrus). - Gemminger \& Harold, 1876:3689. - Kuhnt, 1909:60; 1911:41. - Blackwelder, 1945:465. Distr.: Guiana Francesa. Brasil, região amazônica.

oblongus Duponchel, 1825:164, pl. 7, fig. 80, 81 (Erotylus). - Lacordaire, 1842:92 (Ischyrus). - Crotch, 1876:422 (syn.).

zonalis Lacordaire, 1842:102. Tp.: MCZC exam. aut. - (Bolívia, Guarayos). Crotch, 1876:425 (Megischyrus). - Gemminger \& Harold, 1876:3689. Gorham, 1887:38, pl.2, fig. 16. - Kuhnt, 1909:60; 1911:41. - Bruch, 1914:382. - Mader, 1942d:170; 1951:207. - Blackwelder, 1945:465. Distr.: Da América Central até a Argentina. 
var. lineolatus Crotch, 1876:425. Tp.: MCZC [Localidade não citada]. Gemminger \& Harold, 1876:3689. - Kuhnt, 1909:60; 1911:41. Deelder, 1942:82. - Blackwelder, 1945:465. - Mader, 1951:207. Distr.: Bolívia. Brasil, região amazônica.

ab. imarginata Schenkling, 1919:85. Tp.: DEIB (Colômbia). - Blackwelder, 1845:465. - Mader, 1951:207. Distr.: Colômbia.

\section{Micrischyrus Alvarenga, 1965}

Micrischyrus Alvarenga, 1965:86 (Tgen.: Erotylus quadripunctatus Olivier, 1792: 437, desig. orig.).

Ischyrus; Crotch, 1873, nec Lacordaire, 1842. - Crotch, 1873b:144 (Tgen.: Erotylus quadripunctatus Olivier, 1792:437, seg. Crotch, 1873b:144). Gorham, 1887:39. - Kuhnt, 1909:57, 61. - Deelder, 1942:53. - Boyle, 1956:96, 132. - Alvarenga, 1965:86 (n. nom.).

aleator Boyle, 1954b:46 (Ischyrus). Tp.: CAS (U.S.A., Florida, Cochise County, Chiricahua Mountain); 1956:136, fig. 131, 132. Distr.: U.S.A.

angularis Lacordaire, 1842:126 (Ischyrus). Tp. ? - (Colômbia). - Crotch, 1876: 430. - Gemminger \& Harold, 1876:3690. - Kuhnt, 1909:62; 1911:42. Blackwelder, 1945:465. Distr.: Colômbia.

auriculatus Lacordaire, 1842:123 (Ischyrus). - Tp.: MCZC exam. aut. - (Caiena). - Crotch, 1876:433. - Gemminger \& Harold, 1876:3690. - Kuhnt, 1909:63; 1911:42. - Blackwelder, 1945:465. Distr.: Guiana Francesa. Brasil, região amazônica.

bahiae Crotch, 1876:428 (Ischyrus). Tp.: MCZC exam. aut. - (Brasil, Bahia). Gemminger \& Harold, 1876:3690. - Kuhnt, 1909:63; 1911:42. - Blackwelder, 1945:465. Distr.: Brasil, região sudeste.

bellopictus Kuhnt, 1910:222 (Episcaphula). Tp.: ZMHU exam. aut. (México, Vera Cruz, Motzorongo); 1911:77. - Delkeskamp, 1957:109 (Ischyrus). Distr.: México.

bellus Guérin, 1949a:233, fig. 6 (Ischyrus). Tp.: CDSP (Brasil, Est. São Paulo, São Paulo, Jabaquara). Distr.: Brasil, região sudeste.

bogotae Crotch, 1876:430 (Ischyrus). Tp.: MCZC exam. aut. - (Colômbia, Bogotá). - Gemminger \& Harold, 1876:3690. - Gorham, 1887:40. - Kuhnt, 1909:63; 1911:42. - Blackwelder, 1945:465. Distr.: Guatemala. Colômbia. boucardi Crotch, 1876:429 (Ischyrus). Tp.: MCZC exam. aut. - (Santa Catarina). - Gemminger \& Harold, 1876:3690. - Kuhnt, 1909:64; 1911:42. - Blackwelder, 1945:465. - Guérin, 1948:19. Distr.: Brasil, regiões sudeste e sul. chacojae Gorham, 1887:43, pl. 3, fig. 5 (Ischyrus). Tp.: NHML exam. aut. (Guatemala, Vera Paz, Chacoj). - Kuhnt, 1909:63; 1911:42. - Blackwelder, 1945:465. Distr.: Guatemala. 
chiasticus Boyle, 1954b:43 (Ischyrus). Tp.: CAS (U.S.A., Arizona, Santa Cruz County, Patagonia); 1956:135. Distr.: U.S.A. México.

circumcinctus Delkeskamp, 1957:98, 108 (Ischyrus). Tp.: ZMHU exam. aut. (Peru, Chanchamayo). Distr.: Peru. Bolívia.

collatinus Crotch, 1876:433 (Ischyrus). Tp.: MCZC exam. aut. - (Colômbia). Gemminger \& Harold, 1876:3690. - Gorham, 1887:45. - Kuhnt, 1909:63; 1911:42. - Blackwelder, 1945:465. Distr.: Nicarágua, Colômbia.

conductus Kuhnt, 1910:229, 231 (Ischyrus). Tp.: ZMHU exam. aut. - (Brasil); 1911:42. - Blackwelder, 1945:465. Distr.: Brasil, região sul.

consimilis Crotch, 1876:429 (Ischyrus). Tp.: MCZC exam. aut. - (Brasil, Amazonas). - Gemminger \& Harold, 1876:3690. - Kuhnt, 1909:63:; 1911:42.

- Blackwelder, 1945:465. Distr.: Brasil, região amazônica.

decorus Guérin, 1949a:234, fig. 7 (Ischyrus). Tp.: CDSP exam. aut. - (Brasil, Est. São Paulo, São Paulo, Cantareira). Distr.: Brasil, regiões sudeste e sul.

disconigrum Mader, 1942d:171, 195 (Ischyrus). Tp.: DEIB (Peru, Chanchamayo); 1951:209. Distr.: Peru.

distinguendus Lacordaire, 1842:111 (Ischyrus). Tp. ? - (México). - Crotch, 1876:433. - Gemminger \& Harold, 1876:3690. - Gorham, 1887:45, pl. 2, fig. 23. - Kuhnt, 1909:63; 1911:42. - Blackwelder, 1945:465. Distr.: México.

dunedinensis Leng, 1920:201 (Ischyrus). - Boyle, 1956:136. Distr.: U.S.A.

tripunctatus Blatchley, 1917 nec Crotch, 1873. - Blatchley, 1917:238 (Ischyrus). Tp.: PULI - (U.S.A., Florida, Pinellas County, Dunedin). - Leng, 1920:201 (nom.praeoc.).

duponti Lacordaire, 1842:110 (Ischyrus). Tp. ? - (Colômbia). - Crotch, 1876:426. - Gemminger \& Harold, 1876:3690. - Kuhnt, 1909:63; 1911:42. - Deelder, 1942:82. - Blackwelder, 1945:465. Distr.: Colômbia.

elegantulus Lacordaire, 1842:121 (Ischyrus). Tp. ? - (Colômbia). - Crotch, 1873b:144; 1876:426. - Gemminger \& Harold, 1876:3690. - Gorham, 1887:40. - Kuhnt, 1909:63; 1911:42. - Blackwelder, 1945:465. Distr.: Nicarágua. Colômbia.

ephippiatus Gorham, 1887:43 (Ischyrus). Tp.: NHML exam. aut. - (Panamá, Chiriquí, Bugaba). - Kuhnt, 1909:63; 1911:42. - Blackwelder, 1945:465. Distr.: Panamá.

episcaphulinus Gorham, 1887:44, pl. 3, fig. 7 (Ischyrus). Tp.: NHML exam. aut. - (Guatemala, Zapote). - Kuhnt, 1909:63; 1911:42. - Blackwelder, 1945: 465. Distr.: Guatemala.

femoralis Chevrolat, 1841:63, pl. 18, fig. 10 (Dacne). Tp.: MCZC exam. aut. (México). - Lacordaire, 1842:114 (Ischyrus). - Crotch, 1876:430. - Gemminger \& Harold, 1876:3690. - Gorham, 1887:40. - Kuhnt, 1909:64; 
1911:42. - Blackwelder, 1945:465. Distr.: México.

fraternus Lacordaire, 1842:124 (Ischyrus). Tp.: MCZC exam. aut. - (Colômbia). - Crotch, 1876:428. - Gemminger \& Harold, 1876:3690. - Kuhnt, 1909:62; 1911:42. - Blackwelder, 1945:465. Distr.: Colômbia.

frontalis Lacordaire, 1842:127 (Ischyrus). Tp.: MCZC exam. aut. - (México). Crotch, 1876:426. - Gemminger \& Harold, 1876:3690. - Gorham, 1887: 39. - Kuhnt, 1909:63; 1911:42. - Blackwelder, 1945:465. Distr.: México. Panamá. Colômbia .

agnatus Crotch, 1876:426 (Ischyrus). - Gorham, 1887:39 (syn.).

fulmineus Delkeskamp, 1957:98, 107 (Ischyrus). Tp.: ZSBS (Bolívia, Sarampiuni, San Carlos). Distr.: Bolívia.

grammicus Gorham, 1883:86 (Ischyrus). Tp.: NHML exam. aut. - (Peru, Chanchamayo). - Fleutiaux, 1886:222. - Kuhnt, 1909:63; 1911:42. - Mader, 1942d:171; 1951:210. - Blackwelder, 1945:465. Distr.: Peru.

gratiosus Guérin-Méneville, 1844:310 (Ischyrus). Tp.: MNHN exam. aut.

(Colômbia). - Crotch, 1876:431. - Gemminger \& Harold, 1876:3690. Kuhnt, 1909:62; 1911:42. - Blackwelder, 1945:465. Distr.: Colômbia.

impressopunctatus Crotch, 1876:426 (Ischyrus). Tp.: MCZC exam. aut. - [Localidade não citada]. - Gemminger \& Harold, 1876:3690 Kuhnt, 1909:63; 1911:42. - Blackwelder, 1945:465. - Guérin, 1948:19. Distr.: Brasil, regiões sudeste e sul.

incertus Lacordaire, 1842:118 (Ischyrus). Tp. ? - (Caiena). - Crotch, 1876:431. - Gemminger \& Harold, 1876:3690. - Kuhnt, 1909:62; 1911:42. - Blackwelder, 1945:465. Distr.: Colômbia. Guiana Francesa.

insolens Crotch, 1876:429 (Ischyrus). Tp. ? - (México). - Gemminger \& Harold, 1876:3690. - Gorham, 1887:43, pl. 2, fig. 20. - Kuhnt, 1909:64, pl. 3, fig. 6; 1911:43. - Blackwelder, 1945:465. Distr.: México.

interruptus Duponchel, 1825:48, pl. 1, fig. 27 (Erotylus). Tp. ? - (Brasil). Lacordaire, 1842:116 (Ischyrus). - Crotch, 1876:427. - Gemminger \& Harold, 1876:3690. - Kuhnt, 1909:62; 1911:43. - Blackwelder, 1945:465. Distr.: Brasil, região sudeste.

kempferi Alvarenga, 1977:105 (n. nom.). Distr.: Peru.

nigripes; Mader, 1942, nec Motchulsky, 1858. - Mader, 1942d:171, 195 (Ischyrus). Tp. ? - (Peru, Chanchamayo); 1951:209. - Alvarenga, 1977: 105 (nom.praeoc.).

laetus Lacordaire, 1842:122 (Ischyrus). Tp. ? - (Brasil, Rio de Janeiro). - Crotch, 1876:431. - Gemminger \& Harold, 1876:3691. - Kuhnt, 1909:62; 1911:43.

- Blackwelder, 1945:465. Distr.: Brasil, região sudeste.

lineatus Kuhnt, 1910:228, 231 (Ischyrus). Tp.: ZMHU exam. aut. - (Brasil, Goiás, Jataí); 1911:43. - Blackwelder, 1945:465. Distr.: Brasil, região central. macularis Lacordaire, 1842:128 (Ischyrus). Tp.: MCZC exam. aut. - (Colômbia). 
- Crotch, 1876:430. - Gemminger \& Harold, 1876:3691. - Kuhnt, 1909:62; 1911:43. - Blackwelder, 1945:465. Distr.: Colômbia.

natalensis Guérin, 1956:61, fig. 24 (Ischyrus). Tp.: CDSP exam. aut. - (Brasil, Rio Grande do Norte, Natal, Parnamirim). Distr.: Brasil, região nordeste. nigrolineatus Crotch, 1876:432 (Ischyrus). Tp.: MCZC exam. aut. - [Localidade não citada]. - Gemminger \& Harold, 1876:3691. - Kuhnt, 1909:63; 1911:43. - Blackwelder, 1945:465. - Guérin, 1946b:370. Distr.: Brasil, região sul.

nitidior Crotch, 1876:430 (Ischyrus). Tp.: MCZC exam. aut. - (Brasil, Amazonas). - Gemminger \& Harold, 1876:3691. - Kuhnt, 1909:63; 1911:43. Blackwelder, 1945:465. Distr.: Brasil, região amazônica.

nobilis Crotch, 1876:429 (Ischyrus). Tp.: MCZC exam. aut. - (Brasil, Amazonas). - Fleutiaux, 1886:222. - Kuhnt, 1909:63; 1911:43. - Blackwelder, 1945: 465. Distr.: Brasil, região amazônica.

palliatus Lacordaire, 1842:113 (Ischyrus). Tp. ? - (Colômbia). - Crotch, 1876: 431. - Gemminger \& Harold, 1876:3691. - Kuhnt, 1909:63; 1911:43. Blackwelder, 1945:465. Distr.: Colômbia. Brasil, região amazônica.

pardalinus Guérin, 1949a:235, fig. 8 (Ischyrus). Tp.: CDSP exam. aut. - (Brasil, Paraná, Porto Cabral). Distr.: Brasil, região sul. Argentina.

patruelis Lacordaire, 1842:124 (Ischyrus). Tp. ? - (Brasil, Rio de Janeiro). Crotch, 1876:431. - Gemminger \& Harold, 1876:3691. - Kuhnt, 1909:62; 1911:43. - Blackwelder, 1945:465. Distr.: Brasil, região sudeste.

peruae Mader, 1942d:171, 196 (Ischyrus). Tp.: MGFM exam. aut. - (Peru, Callanga); 1951:210. Distr.: Equador. Peru.

peruvianus Gorham, 1883:85 (Ischyrus). Tp.: NHML exam. aut. - (Peru). Fleutiaux, 1886:222. - Kuhnt, 1909:63; 1911:43. - Mader, 1942d:171; 1951:209. - Blackwelder, 1945:465. Distr.: Peru.

pictus Gorham, 1887:42, pl. 3, fig. 4 (Ischyrus). Tp.: NHML exam. aut. (Guatemala, Vera Paz, San Juan). - Kuhnt, 1909:63; 1911:43. - Blackwelder, 1945:465. - Pallister, 1955b:7. Distr.: México, Guatemala.

proximus Lacordaire, 1842:113 (Ischyrus). Tp.: MCZC exam. aut. - (México). Crotch, 1876:430. - Gemminger \& Harold, 1876:3691. - Gorham, 1887: 40, pl. 2, fig. 21, 22. - Kuhnt, 1909:62; 1911:43. - Blackwelder, 1945:465. Distr.: México. América Central.

puncticollis Gorham, 1887:44 (Ischyrus). Tp.: NHML exam. aut. - (México, Chihuahua, Paso del Norte). - Kuhnt, 1909:63; 1911:43. - Blackwelder, 1945:465. Distr.: México.

quadripunctatus graphicus Lacordaire, 1842:125 (Ischyrus). Tp.: MCZC exam. aut. - (México). - Crotch, 1873b:144; 1876:427. - Gemminger \& Harold, 1876:3690. - Gorham, 1887:39, pl. 2, fig. 17. - Kuhnt, 1909:63; 1911:42. - Blackwelder, 1945:465. - Boyle, 1954b:41; 1956:133, 135. - Pallister, 
1955a:4. Distr.: México. América Central.

quadripunctatus quadripunctatus Olivier, 1792:437 (Erotylus). Tp. ? - (U.S.A., Georgia); 1807:484, pl. 3, fig. 37. - Duponchel, 1825:175. - Sturm, 1826:139; 1843:305 (Mycotretus). - Lacordaire, 1842:127 (Ischyrus). LeConte, 1856:158. - Crotch, 1873a:353; 1876:426. - Gemminger \& Harold, 1876:3691. - Gorham, 1887:39. - Wickham, 1894:341, fig. 61. Kuhnt, 1909:62; 1911:43. - Leng, 1920:201. - Chagnon, 1935:156. Deelder, 1942:82. - Blackwelder, 1945:465. - Froeschner and Meiners, 1953:21, 23. - Boyle, 1954b:39. 1956:133, 152, fig. 126-130. Distr.: Canadá. U.S.A. Guatemala.

quinquepunctatus Gorham, 1887:43, pl. 3, fig. 6 (Ischyrus). Tp.: NHML exam. aut. - (Panamá, Bugaba). - Kuhnt, 1909:63; 1911:43. - Blackwelder, 1945:465. Distr.: Panamá.

rubens Gistel, 1848:124 (Ischyrus). Tp. ? - [Localidade não citada].

scriptus Olivier, 1807:484, pl. 3, fig. 38 (Erotylus). Tp. ? - (Guiana Francesa). Duponchel, 1825:175. - Sturm, 1826:139; 1843:305 (Mycotretus). - Lacordaire, 1842:119 (Ischyrus). - Crotch, 1876:431. - Gemminger \& Harold, 1876:3691. - Kuhnt, 1909:64; 1911:43. - Blackwelder, 1945:465. - Guérin, 1948: 19. Distr.: Guiana Francesa. Brasil, região sudeste.

affinis Duponchel, 1825:47, pl. 1, fig. 26 (Erotylus). Lacordaire, 1842:119 (syn.).

var. maculiventris Lacordaire, 1842:120 (Ischyrus). Tp. ? - (Brasil). - Crotch, 1876:431. - Gemminger \& Harold, 1876:3691. - Kuhnt, 1909:64; 1911:43. - Blackwelder, 1945:465. Distr.: Brasil.

scutellaris Gorham, 1887:41, pl. 3, fig. 2 (Ischyrus). Tp.: NHML exam. aut. (Panamá, Bugaba). - Kuhnt, 1909:63; 1911:43. - Blackwelder, 1945:465. Distr,: Panamá.

septemsignatus Gorham, 1887:41, pl. 2, fig. 19 (Ischyrus). Tp.: NHML exam. aut. - (México, Toxpam). - Kuhnt, 1909:63; 1911:43. - Blackwelder, 1945:465. Distr.: México. Guatemala.

sheppardi Crotch, 1876:428 (Ischyrus). Tp.: MCZC exam. aut. - (Brasil). Gemminger \& Harold, 1876:3691. - Kuhnt, 1909:63; 1911:43. - Blackwelder, 1945:465. Distr.: Brasil, região sudeste.

similior Crotch, 1876:432 (Ischyrus). Tp.: MCZC exam. aut. - [Localidade não citada]. - Gemminger \& Harold, 1876:3691. - Kuhnt, 1909:63; 1911:43. - Blackwelder, 1945:465. - Guérin, 1946b:370; 1948:19. Distr.: Brasil, regiões sudeste e sul.

subcylindricus Lacordaire, $1842: 117$ (Ischyrus). Tp. ? - (Caiena). - Crotch, 1876:428. - Gemminger \& Harold, 1876:3691. - Kuhnt, 1909:64; 1911:43. - Blackwelder, 1945:465. - Delkeskamp, 1957:98. - Deelder, 1942:82. Distr.: Colômbia. Suriname. Guiana Francesa. Bolívia.

tetragrammus Kuhnt, 1910:230, fig. 5 (Ischyrus).Tp.: ZMHU exam. aut. - 
(Argentina, Tucumán); 1911:43. - Bruch, 1914:382. - Blackwelder, 1945: 465. Distr.: Brasil, regiões sudeste e sul. Argentina.

tetraspilotus Guérin-Méneville, 1844:310 (Ischyrus). Tp. ? -(Colômbia). - Crotch,

1876:427. - Gemminger \& Harold, 1876:3691. - Kuhnt, 1909:62; 1911:43.

- Blackwelder, 1945:465. Distr.: Colômbia.

tetrasticus Gorham, 1887:41, pl. 3, fig. 1 (Ischyrus). Tp.: NHML exam. aut. (Panamá, David). - Kuhnt, 1909:64; 1911:43. - Blackwelder, 1945:465.

Distr.: Panamá.

tripunctatus Crotch, 1873b: 144 (Ischyrus). Tp.: MCZC exam. aut. - (Nicarágua, Chontales, Santo Domingo); 1876:427. - Gemminger \& Harold, 1876: 3691. - Gorham, 1887:39. - Kuhnt, 1909:63; 1911:44. - Blackwelder, 1945:465. Distr.: Nicarágua.

undulatus Gorham, 1887:42, pl. 3, fig. 3 (Ischyrus). Tp.: NHML exam. aut. (Panamá, Bugaba). - Kuhnt, 1909:63; 1911:44. - Blackwelder, 1945:465. Distr.: Panamá.

variabilis Duponchel, 1825:46, pl. 1, fig. 25 (Erotylus). Tp. ? - (Brasil). Lacordaire, 1842:121 (Ischyrus). - Crotch, 1876:432. - Gemminger \& Harold, 1876:3691. - Kuhnt, 1909:62; 1911:44. - Bruch, 1914:382. Blackwelder, 1945:465. - Guérin, 1948:19. Distr.: Brasil, regiões sudeste e sul. Argentina.

velatus Lacordaire, 1842:115 (Ischyrus). Tp. ? - (Colômbia). - Crotch, 1876:431.

- Gemminger \& Harold, 1876:3691. - Kuhnt, 1909:63; 1911:44. - Mader, 1942d:171; 1951:209. - Blackwelder, 1945:465. Distr.: Colômbia. Venezuela. Peru.

vespertilio Lacordaire, 1842:112 (Ischyrus). Tp. ? - (Colômbia). - Crotch, 1876:426. - Gemminger \& Harold, 1876:3691. - Gorham, 1887:40. Kuhnt, 1909:63; 1911:44. - Blackwelder, 1945:465. Distr.: Colômbia. México.

vittatus Crotch, 1876:427 (Ischyrus). Tp.: MCZC exam. aut. - (Brasil). - Gemminger \& Harold, 1876:3691. - Kuhnt, 1909:63; 1911:44. - Blackwelder, 1945:465. Distr.: Brasil, região sudeste.

\section{Epytus Dejean, 1837}

Epytus Dejean, 1837:428 (Tgen.: Erotylus cyaneus Duponchel, 1825:60, seg. Crotch, 1876:433). - Crotch, 1876:433. - Alvarenga, 1965:84.

Oocyanus Hope, 1841:113 (Tgen.: Erotylus cyaneus Duponchel, 1825, desig. orig.). - Lacordaire, 1842:31, 194. - Chapuis, 1876:31, 34. - Kuhnt, 1909:56, 64. - Deelder, 1942:52. - Curran, 1944:1. - Alvarenga, 1965:88 (syn.).

cyaneus Duponchel, 1825:60, pl. 2, fig. 56 (Erotylus). Tp. ? - [Localidade não citada]. - Curran, 1944:2, 5, fig. 7 (Oocyanus). - Distr.: Cuba. 
violaceus Sturm, 1826:81, pl. 4, fig. 38 (Erotylus). - Lacordaire, 1842:196 (Oocyamus). - Curran, 1944:5 (syn.).

flavitarsis Lacordaire, 1842:130 (Ischyrus). Tp. ? - (Cuba). - Jacquelin du Val, 1857:321, pl. 10, fig. 11. - Crotch, 1876:432. - Gemminger \& Harold, 1876:3690. - Kuhnt, 1909:62; 1911:42. - Deelder, 1942:82. - Blackwelder, 1945:465. - Sturm, 1843:305 (Mycotretus). - Curran, 1944:2, 3, fig. 2 (Oocyanus). - Candèze, 1861:394, pl. 6, fig. 5. Distr.: Cuba. Haiti. República Dominicana. Bahamas.

fulvitarsis Lacordaire, 1842:129 (Ischyrus). Tp.: MCZC exam. aut. - (Haiti). Crotch, 1876:432. - Gemminger \& Harold, 1876:3690. - Kuhnt, 1909:62; 1911:42. - Blackwelder, 1945:465. - Curran, 1944:2, 3, figs 4, 5 (Oocyanus). Distr.: Cuba. Haiti. República Dominicana.

brunnipes Kuhnt, 1910:231 (Oocyanus). - Curran, 1944:3 (syn.).

haitensis Curran, 1944:2, fig. 1. (Oocyanus). Tp.: AMNH exam. aut. - (Haiti, La Hotte). Distr.: Haiti.

modestus Olivier, 1807:483, pl. 3, fig. 35 (Erotylus). Tp. ? - (S. Domingo). Duponchel, 1825:165, pl. 7, fig. 82. - Lacordaire, 1842:130 (Ischyrus). Crotch, 1876:432. - Gemminger \& Harold, 1876:3691. - Kuhnt, 1909:62; 1911:43. - Blackwelder, 1945:465. - Curran, 1944:2, 3, fig. 3 (Oocyanus). Distr.: Haiti. República Dominicana.

tarsalis Lacordaire, 1842:106 (Ischyrus). Tp. ? - (Haiti). - Gemminger \& Harold, 1876:3696 (Oocyanus). - Kuhnt, 1909:64; 1911:44. - Blackwelder, 1945:465. - Curran, 1944:2, 4, fig. 6. Distr.: Cuba. Haiti.

tarsatus Lacordaire, 1842:196 (Oocyanus). Crotch, 1876:433. (Epytus). Curran, 1944:4 (syn.).

\section{Callischyrus Crotch, 1876}

Callischyrus Crotch, 1876:434 (Tgen.: Erotylus insignis Laporte, 1840:520, seg. Crotch, 1876:434). - Gorham, 1887:45. - Kuhnt, 1909:57, 64. - Deelder, 1942:53. - Alvarenga, 1965:82.

amoenus Guérin-Méneville, 1841b: 155 (Lybas). Tp.: MCZC exam. aut. - (México). - Lacordaire, 1842:107 (Ischyrus). - Gemminger \& Harold, 1876: 3690. - Crotch, 1876:435 (Callischyrus). - Gorham, 1887:46, pl. 2, fig. 25. - Kuhnt, 1909:65, pl. 3, fig. 7; 1911:44. - Deelder, 1942:83. Blackwelder, 1945:465. Distr.: México. Costa Rica.

apicatus Kuhnt, 1910:231. Tp. ? - (Equador); 1911:44. - Blackwelder, 1945:465.

Distr.: Equador.

brasiliensis Deelder, 1942:83, fig. 2c. Tp.: RNHL (Brasil, Est. São Paulo). Distr.: Brasil, regiões sudeste e sul.

candezei Crotch, 1876:434. Tp.: MCZC exam. aut. - (Guatemala). - Gemminger 
\& Harold, 1876:3690 (Ischyrus). - Gorham, 1887:46, pl. 2, fig. 24 (Callischyrus). - Kuhnt, 1909:65; 1911:44. - Deelder, 1942:83. - Blackwelder, 1945:465. Distr.: Guatemala.

cyanopterus boliviensis Delkeskamp, 1957:98, 109. Tp.: ZSBS - (Bolívia, Sihuencas, Yungas de Arepucho). Distr.: Bolívia.

cyanopterus cyanopterus Erichson, 1847:180 (Ischyrus). Tp.: ZMHU exam. aut. - (Peru). - Gemminger \& Harold, 1876:3690. - Crotch, 1876:434 (Callischyrus). - Kuhnt, 1909:65; 1911:44. - Mader, 1942d:171; 1951:210. Blackwelder, 1945:465. Distr.: México. Colômbia. Equador. Peru.

hieroglyphicus Duponchel, 1825:46, pl. 1, fig. 24 (Erotylus). Tp. ? - (Brasil). Lacordaire, 1842:106 (Ischyrus). - Gemminger \& Harold, 1876:3690. Crotch, 1876:435 (Callischyrus). - Kuhnt, 1909:65; 1911:44. - Bruch, 1914:384. - Deelder, 1942:83. - Blackwelder, 1945:465. Distr.: Brasil, regiões sudeste e sul. Argentina.

insignis Laporte, 1840,520 (Erotylus). Tp.: MCZC exam. aut. - (Colômbia). Lacordaire, 1842:108 (Ischyrus). - Gemminger \& Harold, 1876:3690. Crotch, 1876:434 (Callischyrus). - Kuhnt, 1909:65; 1911:44. - Deelder, 1942:83. - Mader, 1942d:171; 1951:210. - Blackwelder, 1945:465. Distr.: Venezuela. Colômbia. Equador. Peru.

elegans Guérin-Méneville, 1841:118 (Sternolobus). - Lacordaire, 1842:108 (syn.).

blandus Erichson, 1847:180 (Ischyrus). - Crotch, 1876:434 (syn.).

melanogaster Guérin-Ménevile, 1844:310 (Ischyrus). Tp. MCZC exam. aut. (Colômbia). - Gemminger \& Harold, 1876:3691. - Crotch, 1876:434 (Callischyrus). - Kuhnt, 1909:65; 1911:45. - Blackwelder, 1945:465. Distr.: Colômbia.

melanopus Guérin-Méneville, 1841a:118 (Morphoides). Tp. ? - (Colômbia). Lacordaire, 1842:109 (Ischyrus). - Gemminger \& Harold, 1876:3691. Crotch, 1876:434 (Callischyrus). - Kuhnt, 1909:65; 1911:45. - Blackwelder, 1945:465. Distr.: Colômbia.

praenobilis Delkeskamp, 1957:110, fig. 7. Tp.: ZMHU exam. aut. - (Colômbia, Volcancito, Paso des Quindiu). Distr.: Colômbia.

venustus Lacordaire, 1842:109 (Ischyrus). Tp. ? - (Colômbia). Guérin-Méneville, 1855:610. - Gemminger \& Harold, 1876:3691. - Crotch, 1876:434 (Callischyrus). - Gorham, 1887:46. - Kuhnt, 1909:65; 1911:45. - Deelder, 1942:83. - Blackwelder, 1945:465. Distr.: México. Colômbia. Equador. 


\section{Cyrtomorphus Lacordaire, 1842}

Cyrtomorphus Lacordaire, 1842:240 (Tgen.: Cyrtomorphus pantherinus Lacordaire, 1842:241, seg. Crotch, 1876: 436). - Crotch, 1876:436. - Kuhnt, 1909:56, 68. - Delkeskamp, 1981:38.

pusillus Kirsch, 1876:102. Tp. ? - (Peru, Pozuzo). - Kuhnt, 1909:69; 1911:47. Distr.: Peru.

\section{Mycotretus Chevrolat, 1837}

Mycotretus Chevrolat, 1837:428 (Tgen.: Erotylus ornatus Duponchel, 1825:49, seg. Gorham, 1887:46). - Lacordaire, 1842:31, 132. - Crotch, 1873a:354; 1873b:144; 1876:437. - Chapuis, 1876:31, 32. - Gorham, 1887:46. - Kuhnt, 1909:57, 70. - Casey, 1916:158. - Deelder, 1942:54. - Mader, 1951:210. Guérin, 1952:180. - Boyle, 1956:97, 137. - Alvarenga, 1965:87.

Lybas Chevrolat, 1837:429 (Tgen.: Erotylus lesueri Chevrolat, 1835:175, seg. Alvarenga, 1965:85). - Alvarenga, 1965:85 (syn.).

adalioides Crotch, 1876:448. Tp.: MCZC exam. aut. - (Peru). - Gemminger \& Harold, 1876:3691. - Kuhnt, 1909:74; 1911:47. - Mader, 1942d:173; 1951:214. - Blackwelder, 1945:465. Distr.: Peru.

aegrotus Gorham, 1888:60. Tp.: NHML - (Costa Rica, Volcán de Irazú). - Kuhnt, 1909:73; 1911:47. - Blackwelder, 1945:465. Distr.: Costa Rica.

aestuans Lacordaire, 1842:170. Tp. ? - (Brasil). - Crotch, 1876:441. - Gemminger \& Harold, 1876:3691. - Kuhnt, 1909:76; 1911:48. - Blackwelder, 1945: 465. Distr.: Brasil.

alternans Gorham, 1888:57. Tp.: NHML exam. aut. - (Panamá, Bugaba). - Kuhnt, 1909:75; 1911;48. - Blackwelder, 1945:465. Distr.: Panamá.

ambulator Lacordaire, 1842:175. Tp. ? - (Caiena). - Crotch, 1876:457. - Gemminger \& Harold, 1876:3691. - Kuhnt, 1909:72; 1911:48. - Blackwelder, 1945:465. Distr.: Guiana Francesa.

anchoralis Guérin, 1956:62, fig. 25. Tp.: CDSP exam. aut. - (Argentina, Jujuy, Reyes). Distr.: Bolívia, Argentina.

antesignatus Mader, 1942d:174, 198. Tp.: NKMS - (Peru); 1951:220. Distr.: Peru.

apicalis Lacordaire, 1842:181. Tp. ? - (Colômbia). - Crotch, 1876:448. Gemminger \& Harold, 1876:3691. - Kuhnt, 1909:74; 1911:48. - Mader, 1942d:173; 1951:214. - Blackwelder, 1945:465. Distr.: Colômbia. Peru.

arcuatus Lacordaire, 1842:158. Tp. ? - (Caiena). - Crotch, 1876:437. - Gemminger \& Harold, 1876:3692. - Kuhnt, 1909:71; 1911:48. - Blackwelder, 1945:465. Distr.: Guiana Francesa. 
argus Lacordaire, 1842:161. Tp. ? - (Caiena). - Crotch, 1876:439. - Gemminger \& Harold, 1876:3692. - Kuhnt, 1909:71; 1911:48. - Blackwelder, 1945: 465. Distr.: Guiana Francesa.

atricaudatus Gorham, 1888:66, pl. 4, fig. 4. Tp.: NHML exam. aut. - (Panamá, Volcán de Chiriquí). - Kuhnt, 1909:71; 1911:48. - Blackwelder, 1945:465. Distr.: Panamá.

badius Gorham, 1888:70. Tp.: NHML exam. aut. - (Guatemala, Vera Paz, San Juan). - Kuhnt, 1909:71; 1911:48. - Blackwelder, 1945:465. Distr.: Guatemala.

balteatus Crotch, 1876:445. Tp.: MCZC exam. aut. - (Ega)[atualmente, Tefé]. Gemminger \& Harold, 1876:3692. - Kuhnt, 1909:74; 1911:48. - Blackwelder, 1945:465. Distr.: Brasil, região amazônica.

basalis Crotch, 1876:438. Tp. MCZC exam. aut. - (Ega)[atualmente, Tefé] Gemminger \& Harold, 1876:465. - Kuhnt, 1909:76; 1911:52. Distr.: Brasil, região amazônica.

bicinctus Guérin, 1949a:236, fig. 9. Tp.: CDSP - (Brasil, Est. S. Paulo, São Paulo, Cantareira). Distr.: Brasil, regiões sudeste e sul.

bicolor Taschenberg, 1870:198. Tp. ? - (Colômbia). - Gemminger \& Harold, 1876:3692. - Kuhnt, 1909:73; 1911:48. - Mader, 1942d:172; 1951:213. Balckwelder, 1945:465. Distr.: Colômbia. Ecuador. Peru.

bicoloratus Kuhnt, 1911:48. - Mader, 1942d:172; 1951:211. - Blackwelder, 1945:465. Distr.: Peru.

bicolor; Kirsch, 1876, nec Taschenberg, 1870. - Kirsch, 1876:101. Tp. ? (Peru, Pozuzo). - Fleutiaux, 1886:223. - Kuhnt, 1909:72; 1911:48 (nom.praeoc.).

bipunctatus Gorham, 1888:58. Tp.: NHML exam. aut. - (Panamá, Bugaba). Kuhnt, 1909:73; 1911:48. - Blackwelder, 1945:466. Distr.: Panamá.

bisseptemguttatus Crotch, 1876:441. Tp.: MCZC exam. aut. - (Caiena). - Gemminger \& Harold, 1876:3692. - Kuhnt, 1909:71; 1911:48. - Blackwelder, 1945:466. Distr.: Guiana Francesa.

bistrigatus Lacordaire, 1842:188. Tp. ? - (México). - Crotch, 1876:456. Gemminger \& Harold, 1876:3692. - Gorham, 1888:52; 1898:253. - Kuhnt, 1909:75; 1911:48. - Blackwelder, 1945:466. Distr.: México.

bistrioculatus Alvarenga, 1983:583. Tp.: CARJ - (Brasil, Amazonas, Rio Javari, Estirão do Equador). Distr.: Brasil, região amazônica.

brasilianus Crotch, 1876:442. Tp.: MCZC-(Brasil).-Fleutiaux, 1886:223.-Kuhnt, 1909:74; 1911:48. - Blackwelder, 1945:466. Distr.: Brasil, região sudeste.

brevis Gorham, 1888:67. Tp.: NHML - (Panamá, Bugaba). - Kuhnt, 1909:71; 1911:48. - Blackwelder, 1945:466. Distr.: Panamá.

centralis Arrow, 1909:196. - Kuhnt, 1911:54. - Mader, 1942d:174; 1951:216. Blackwelder, 1945:468. Distr.: México. Guatemala. 
tigrinus; Gorham, 1887, nec Olivier, 1792. Tp.: NHML exam. aut. (México, Toxpam). - Arrow, 1909:196 (n. nom.).

cercyonoides Gorham, 1888:67. Tp.: NHML exam. aut. - (Panamá, David). Kuhnt, 1909:74; 1911:48. - Blackwelder, 1945:466. Distr.: Panamá.

chilensis Crotch, 1876:454. Tp.: MCZC exam. aut. - (Chile). - Gemminger \& Harold, 1876:3692. - Kuhnt, 1909:75; 1911:48. - Blackwelder, 1945:466.

- Guérin, 1952:182. Distr.: Chile.

chontalesi Crotch, 1873b:145. Tp.: MCZC exam. aut. - (Nicarágua, Chontales); 1876:446. - Gemminger \& Harold, 1876:3692. - Gorham, 1888:70. Kuhnt, 1909:72; 1911:48. - Blackwelder, 1945:466. Distr.: Nicarágua.

cinctellus Guérin-Méneville, 184 lb:153 (Brachymerus). Tp.: MNHN exam. aut. - (Bolívia). - Lacordaire, 1842:178 (Mycotretus). - Crotch, 1876:447. Gemminger \& Harold, 1876:3692. - Kuhnt, 1909:71; 1911:48. - Bruch, 1914:384. - Deelder, 1942:88. - Mader, 1942d:172; 1951:212. - Blackwelder, 1945:466. Distr.: Da América Central até a Argentina.

cinctiger Crotch, 1876:438. Tp.: MCZC exam. aut. - (Santarém). - Gemminger \& Harold, 1876:3692. - Kuhnt, 1909:75; 1911:48. - Mader, 1942d:174; 1951:218. - Balckwelder, 1945:466. Distr.: Brasil, região amazônica.

clitelliger Lacordaire, 1842:149. Tp. ? - (Colômbia). - Crotch, 1876:452. Gemminger \& Harold, 1876:3692. - Kuhnt, 1909:74; 1911:48. - Blackwelder, 1945:466. - Mader, 1951:217. Distr.: Colômbia.

coccidulinus Gorham, 1888:63, pl. 3, fig. 24. Tp.: NHML - (British Honduras, Belize, R. Hondo). - Kuhnt, 1909:72; 1911:48. - Blackwelder, 1945:466.

- Guérin, 1946:370. - Distr.: Belize. Guatemala.

coccinelloides Taschenberg, 1870:198. Tp. ? - (Colômbia). - Gemminger \& Harold, 1876:3692. - Kuhnt, 1909:74; 1911:48. - Blackwelder, 1945:466. Distr.: Colômbia.

coccineus Lacordaire, 1842:239 (Lybas). Tp. ? - (Rio de Janeiro). - Crotch, 1876:458 (Mycotretus). - Gemminger \& Harold, 1876:3692. - Kuhnt, 1909:72; 1911:48. - Curran, 1941:286. - Deelder, 1942:88. - Blackwelder, 1945:466. Distr.: Guiana. Suriname. Brasil, regiões amazônica e sudeste. coelestinus Lacordaire, 1842:170. Tp. ? - (Colômbia). - Crotch, 1876:439. Gemminger \& Harold, 1876:3692. - Kuhnt, 1909:74; 1911:48. - Blackwelder, 1945:466. Distr.: Colômbia.

cognatus Lacordaire, 1842:145. Tp. ? - (Rio de Janeiro). - Crotch, 1876:450. Gemminger \& Harold, 1876:3692. - Kuhnt, 1909:75; 1911:48. - Blackwelder, 1945:466. Distr.: Brasil, região sudeste.

consanguineus Gorham, 1888:61, pl. 3, fig. 23. Tp.: NHML - (Guatemala, Cubilguitz). - Kuhnt, 1909:72; 1911:48. - Blackwelder, 1945:466. Distr.: Guatemala. Panamá.

corallipennis Crotch, 1876:448. Tp. ? - (Venezuela). - Gemminger \& Harold, 
1876:3692. - Kuhnt, 1909:71; 1911:48. - Blackwelder, 1945:466: Distr.: Colômbia. Venezuela.

cordiger Crotch, 1876:455. Tp.: MCZC exam. aut. - (Ega)[atualmente, Tefé]. Gemminger \& Harold, 1876:3692. - Kuhnt, 1909:75; 1911:48. - Blackwelder, 1945:466. Distr.: Brasil, região amazônica.

coronatus Duponchel, 1825:50, pl. 2, fig. 33 (Erotylus). Tp. ? - (Brasil). Lacordaire, 1842:141 (Mycotretus). - Crotch, 1876:450. - Gemminger \& Harold, 1876:3692. - Kuhnt, 1909:75; 1911:48. - Blackwelder, 1945:466.

Distr.: Brasil, Minas Gerais.

cribratus Gorham, 1888:64. Tp.: NHML exam. aut. - (Guatemala, Senahú). Kuhnt, 1909:73; 1911:48. - Blackwelder, 1945:466. Distr.: Guatemala. Panamá.

cruciger Crotch, 1876:452. Tp.: MCZC exam. aut. - [Localidade não citada]. Gemminger \& Harold, 1876:3692. - Kuhnt, 1909:74; 1911:49. - Blackwelder, 1945:466. Distr.: Patria?

crudus Gorham, 1888:63. Tp.: NHML - (México, Puebla, Atlisco). - Kuhnt, 1909:73; 1911:49. - Blackwelder, 1945:466. Distr.: México.

cruentus Gorham, 1888:59, pl. 3, fig. 22. Tp.: NHML - (Guatemala, Zapote). Kuhnt, 1909:73; 1911:49. - Blackwelder, 1945:466. Distr.: Guatemala.

cunctans Mader, 1942d: 174, 198. Tp.: SMTD exam. aut. -(Peru, Chanchamayo); 1951:217. Distr.: Peru.

cyanopterus Lacordaire, 1842:179. Tp.: MCZC exam. aut. - (Colômbia).

Crotch, 1876:447. - Gemminger \& Harold, 1876:3692. - Kuhnt, 1909:73; 1911:49. - Blackwelder, 1945:466. Distr.: Colômbia.

decoratus Duponchel, 1825:50, pl. 2, fig. 32 (Erotylus). Tp. ? - (Brasil). Lacordaire, 1842:172 (Mycotretus). - Crotch, 1876:442. - Gemminger \& Harold, 1876:3692. - Kuhnt, 1909:76; 1911:49. - Blackwelder, 1945:466. Distr.: Brasil, região amazônica.

decorus Crotch, 1876:442. Tp.: MCZC exam. aut. - (Ega)[atualmente Tefé]. Gemminger \& Harold, 1876:3692. - Kuhnt, 1909:76; 1911:49. - Blackwelder, 1945:466. Distr.: Brasil, região amazônica.

derasofasciatus Kuhnt, 1910:237. fig. 7. Tp. ? - (Peru, Chanchamayo); 1911:49. - Mader, 1942d:175, 199; 1951:219, 222. - Blackwelder, 1945:466. Guérin, 1949:236. Distr.: Venezuela. Bolívia. Peru. Brasil, região amazônica.

deyrollei Crotch, 1876:451. Tp. ? - (Brasil, Santa Catarina). - Gemminger \& Harold, 1876:3692. - Kuhnt, 1909:75; 1911:49. - Blackweler, 1945:466.

Distr.: Brasil, região sul.

dichrous Kirsch, 1876:100. Tp.: SMTD exam. aut. -(Peru, Pozuzu). - Gemminger \& Harold, 1876:3692. - Kuhnt, 1909:71; 1911:49. - Mader, 1942d:172; 1951: 212.-Blackwelder, 1945:466. Distr.: Peru. Brasil, região amazônica. 
difficilis Lacordaire, 1842:136. Tp. ? - (Brasil). - Crotch, 1876:449. - Gemminger \& Harold, 1876:3692. - Kuhnt, 1909:76; 1911:49. - Blackwelder, 1945: 466. Distr.: Brasil.

dimidiatus Taschenberg, 1870:198. Tp. ? - (Colômbia) - Gemminger \& Harold, 1876:3692. - Kuhnt, 1909:71; 1911:49. - Blackwelder, 1945:466. Distr.: Colômbia.

discipennis Kuhnt, 1910:240, fig. 9. Tp. ? - (Brasil, Santa Catarina); 1911:49. Blackwelder, 1945:466. Distr.: Brasil, região sul.

var. conductus Kuhnt, 1910:240. Tp. ? - (Brasil, Santa Catarina); 1911:49. Blackwelder, 1945:466. Distr.: Brasil, região sul.

discoidalis Taschenberg, 1870: 199. Tp. ? - (Colômbia). - Gemminger \& Harold, 1876:3692. - Kuhnt, 1909:73; 1911:49. - Mader, 1942d:173; 1951:214. Blackwelder, 1945:466. Distr.: Colômbia. Peru.

dispar Taschenberg, 1870:197. Tp. ? - (Colômbia). - Gemminger \& Harold, 1876:3692. - Kuhnt, 1909:74; 1911:49. - Deelder, 1942:88. - Mader, 1942d:173; 1951:215, 223. - Blackwelder, 1945:466. Distr.: Colômbia. Ecuador. Peru.

distigma Lacordaire, 1842:190. Tp. ? - (Colômbia). - Crotch, 1876:457. Gemminger \& Harold, 1876:3692. - Kuhnt, 1909:74; 1911:49. - Blackwelder, 1945:466. Distr.: Colômbia.

distinguendus Arrow, 1909:196. Tp.: NHML exam. aut. - (México). - Kuhnt, 1911:54. - Blackwelder, 1945:466. Distr.: México.

dorsofasciatus Lacordaire, 1842:173. Tp. ? - (Brasil). - Crotch, 1876:444.

Gemminger \& Harold, 1876:3692. - Kuhtn, 1909:75; 1911:49. - Blackwelder, 1945:466. Distr.: Brasil, região sudeste.

dorsonotatus Lacordaire, 1842:151. Tp. ? - (Caiena). - Crotch, 1876:453. Gemminger \& Harold, 1876:3692. - Kuhnt, 1909:71,75; 1911:49. Curran, 1941:286. - Mader, 1942d:172; 1951:212. - Blackwelder, 1945: 466. Distr.: Guiana. Guiana Francesa. Brasil, região amazônica. Peru.

dubius Lacordaire, 1842:141. Tp. ? - (Brasil). - Crotch, 1876:449. - Gemminger \& Harold, 1876:3692. - Kuhnt, 1909:75; 1911:49. - Blackwelder, 1945: 466. Distr.: Brasil.

duodecimguttatus Duponchel, 1825:53, pl. 2, fig. 38 (Erotylus). Tp. ? - (Brasil). - Lacordaire, 1842:164 (Mycotretus). Distr.: Colômbia. Peru. Brasil, regiões amazônica e sul.

ocellatus; Germar, 1824, nec Olivier, 1792. - Germar, 1824:613 (Erotylus). Tp.: ZMHU exam. aut. - (Brasil). - Crotch, 1876:441 (Mycotretus). Gemminger \& Harold, 1876:3694. - Kuhnt, 1909:74; 1911:51. Mader, 1942d:173; 1951:215. - Deelder, 1942:88. - Blackwelder, 1945:467. - Lacordaire, 1842:164 (nom.praeoc.).

var. consociatus Kuhnt, 1910:238. Tp.? - (Colômbia, Bogotá); 1911:51. -Mader, 1942d:173; 1951:215. -Blackwelder, 1945:467. Distr.: Colômbia. 
durius Lacordaire, 1842:161. Tp. ? - (Caiena). - Crotch, 1876:453. - Gemminger \& Harold, 1876:3692. - Kuhnt, 1909:72; 1911:49. - Curran, 1941:286, 287. - Blackwelder, 1945:466. Distr.: Guiana Francesa. Brasil, região amazônica.

dytiscoides Lacordaire, 1842:184. Tp. ? - (México). - Crotch, 1876:449. Gemminger \& Harold, 1876:3692. - Gorham, 1888:65. - Kuhnt, 1909:73; 1911:49. - Blackwelder, 1945:466. Distr.: México.

egae Crotch, 1876:442. Tp.: MCZC exam. aut. - (Ega)[atualmente, Tefé]. Gemminger \& Harold, 1876:3692. - Kuhnt, 1909:74; 1911:49. - Blackwelder, 1945:466. Distr.: Brasil, região amazônica.

elegans Groham, 1888:55, pl. 4, fig. 3. Tp.: NHML exam. aut. - (Guatemala, San Gerónimo). - Kuhnt, 1909:71; 1911:49. - Blackwelder, 1945:466. Distr.: Guatemala.

episcaphoides Crotch, 1876:456. Tp.: MCZC exam. aut. - (Ega) [atualmente Tefé]. - Gemminger \& Harold, 1876:3692. - Kuhnt, 1909:71; 1911:49. Blackwelder, 1945:466. Distr.: Brasil, região amazônica.

episcopalis Lacordaire, 1842:152. Tp. ? - (Brasil). - Crotch, 1876:453. - Gemminger \& Harold, 1876:3692. - Kuhnt, 1909:75; 1911:49. - Blackwelder, 1945:466. Distr.: Guiana Francesa. Brasil, região amazônica.

epopterus Gorham, 1888:69, pl. 4, fig. 7. Tp.: NHML exam. aut. - (México, Toxpam). - Kuhnt, 1909:76; 1911:49. - Blackwelder, 1945:466. Distr.: México.

erraticus Gorham, 1898:254. Tp.: NHML exam. aut. - (México, Guerrero, Chilpancingo). - Kuhnt, 1909:73; 1911:49. - Blackwelder, 1945:466. Distr.: México.

expressus Kuhnt, 1910:237. Tp.: ZMHU exam. aut. - (Brasil, Goiás, Jataí); 1911:49. - Blackwelder, 1945:466. Distr.: Brasil, região central.

fallax Guérin-Méneville, 1841b: 155. Tp.: MNHN exam. aut. - (Brasil). - Lacordaire, 1842:153. - Crotch, 1876:453. - Gemminger \& Harold, 1876:3692. - Kuhnt, 1909:76; 1911:49. - Blackwelder, 1945:466. - Guérin, 1948:19. Distr.: Brasil, região sudeste e sul.

fasciatus Fabricius, 1801:570 (Tritoma). Tp. ? - (América meridional). - Chevrolat, 1843:79 (Mycotretus). Distr.: Patria?

fasciolatus Lacordaire, 1842:150. Tp.: MCZC exam. aut. - (México). - Crotch, 1876:452. - Gemminger \& Harold, 1876:3692. - Gorham, 1888:49. Kuhnt, 1909:75; 1911:49. - Blackwelder, 1945:466. Distr.: México. Guatemala. Panamá.

fascipennis Kuhnt, 1910:244. Tp.: ZMHU exam. aut. - (Suriname); 1911:49. Blackwelder, 1945:466. Distr.: Suriname.

fidelis Delkeskamp, 1939:27. - Mader, 1951:211. Distr.: Peru. 
parallelus; Kuhnt, 1909, nec Crotch, 1876. - Kuhnt, 1909:30 (Brachysphaenus). Tp.: ZMHU - (Peru); - 1911:18. - Mader, 1938:17; 1942d: 171. - Blackwelder, 1945:459. - Delkeskamp, 1939:27 (nom.praeoc. e comb.n.).

flavomarginatus Lacordaire, 1842:157. Tp. ? - (Rio de Janeiro). - Crotch, 1876:444. - Gemminger \& Harold, 1876:3692. - Kuhnt, 1909:7i ; 1911:49.

- Blackwelder, 1945:466. Distr.: Brasil, região sudeste.

floriger Lacordaire, 1842:185. Tp. ? - (Caiena). - Crotch, 1876:456. -Gemminger \& Harold, 1876:3692. - Kuhnt, 1909:71; 1911:49. - Blackwelder, 1945: 466. Distr.: Guiana Francesa. Brasil, região amazônica.

fragosoi Alvarenga, 1983:587. Tp.: CARJ - (Brasil, Amazonas, rio Javari, Estirão do Equador). Distr.: Brasil, região amazônica.

fulviceps Crotch, 1876:447. Tp. ? - (Pará, Santarém). - Gemminger \& Harold, 1876:3693. - Kuhnt, 1909:73; 1911:49. - Blackwelder, 1945:466. Distr.: Brasil, região amazônica.

fulvilabris Crotch, 1876:447. Tp.: MCZC exam. aut. - (Brasil, Amazonas, São Paulo)[atualmente São Paulo de Olivença]. - Gemminger \& Harold, 1876: 3693. - Kuhnt, 1909:71; 1911:49. - Blackwelder, 1945:466. Distr.: Brasil, região amazônica.

fuscitarsis Lacordaire, 1842:180. Tp. ? - (México). - Crotch, 1876:448. Gemminger \& Harold, 1876:3693. - Gorham, 1888:68, pl. 3, fig. 25. Kuhnt, 1909:73; 1911:49. - Blackwelder, 1945:466. Distr.: México e América Central.

geminus Gorham, 1888:50, pl. 3, fig. 10. Tp.: NHML exam. aut. - (Panamá, Bugaba). - Kuhnt, 1909:75; 1911:50. - Blackwelder, 1945:466. Distr.: Panamá.

gemmula Lacordaire, 1842:181. Tp. ? - (Colômbia). - Crotch, 1876:448. Gemminger \& Harold, 1876:3693. - Kuhnt, 1909:73; 1911:50. - Deelder, 1942:88. - Mader, 1942d:173; 1951:214. - Blackwelder, 1945:466. Distr.: Colômbia. Peru.

var. pulicarius Lacordaire, 1842:182. Tp. ? - (Colômbia). - Crotch, 1876:448. - Gemminger \& Harold, 1876:3693. - Mader, 1942d:173. - Blackwelder, 1945:466. Distr.: Colômbia.

gentilis Lacordaire, 1842:182. - Crotch, 1876:448 (syn.).

graniformis Lacordaire, 1842:152. Tp. ? - (Caiena). - Crotch, 1876:453. Gemminger \& Harold, 1876:3693. - Kuhnt, 1909:72; 1911:50. - Blackwelder, 1945:466. Distr.: Guiana Francesa.

graphoderus Lacordaire, 1842:144. Tp.: MCZC exam. aut. - (Brasil). - Crotch, 1876:450. - Gemminger \& Harold, 1876:3693. - Kuhnt, 1909:76; 1911:50.

- Bruch, 1914:384. - Deelder, 1942:88. - Blackwelder, 1945:466. - Mader, 1951:220-221. Distr.: Brasil, regiões sudeste e sul. Argentina.

var. strigipennis Kuhnt, 1910:244. Tp.: ZMHU exam. aut. - [Localidade não 
citada]; 1911:50. - Bruch, 1914:384. - Blackwelder, 1945:466.

Guérin, 1948:20. - Mader, 1951:221. Distr.: Brasil, sudeste e sul. Argentina.

var. thoracicus Kuhnt, 1910:244. Tp.: ZMHU exam. aut. - [Localidade não citada]; 1911:50. - Bruch, 1914:384. - Blackwelder, 1945:466. Guérin, 1948:19. - Mader, 1951:221. Distr.: Brasil, regiões sudeste e sul. Argentina.

guatemalae Crotch, 1876:441. Tp.: MCZC exam. aut. - (Guatemala). - Gorham, 1888:55. - Kuhnt, 1909:72; 1911:50. - Blackwelder, 1945:466. Distr.: Guatemala.

haemapterus Gorham, 1888:68. Tp.: NHML - (Panamá, Bugaba). - Kuhnt, 1909:71; 1911:50. - Blackwelder, 1945:466. Distr.: Panamá. Brasil, região amazônica.

haematicus Gorham, 1888:61. Tp.: NHML - (Costa Rica). - Kuhnt, 1909:72; 1911:50. - Deelder, 1942:88. - Blackwelder, 1945:466. Distr.: Costa Rica. hepaticus Lacordaire, 1842:190. Tp. ? - (Colômbia). - Crotch, 1876:457. Gemminger \& Harold, 1876:3693. - Kuhnt, 1909:72; 1911:50. - Blackwelder, 1945:466. - Distr.: Colômbia.

hilaris Lacordaire, 1842:171. Tp. ? - (Brasil). - Crotch, 1876:441. - Gemminger \& Harold, 1876:3693. - Kuhnt, 1909:76; 1911:50. - Blackwelder, 1945:466. Distr.: Brasil, região sudeste.

hirudo Gorham, 1888:59. Tp.: NHML - (Guatemala, San Gerónimo). - Kuhnt, 1909:73; 1911:50. - Blackwelder, 1945:466. Distr.: Guatemala.

humeralis Germar, 1824:614 (Erotylus). Tp. ? - (Brasil). - Lacordaire, 1842:189 (Mycotretus). - Crotch, 1876:457. - Gemminger \& Harold, 1876:3693. Kuhnt, 1909:74; 1911:50. - Blackwelder, 1945:466. Distr.: Brasil.

humilis Lacordaire, 1842:189. Tp. ? - (Caiena). - Crotch, 1876:457. - Gemminger \& Harold, 1876:3693. - Kuhnt, 1909:73; 1911:50. - Blackwelder, 1945: 466. Distr.: Guiana Francesa.

illustris Crotch, 1876:440. Tp.: MCZC exam. aut. - (México). - Gemminger \& Harold, 1876:3693. - Gorham, 1888:54, pl. 3, fig. 15. - Kuhnt, 1909:72; 1911:50. - Blackwelder, 1945:466. Distr.: México.

incarnatus Gorham, 1888:62. Tp.: NHML - (México, Jalapa). - Kuhnt, 1909:73; 1911:50. - Blackwelder, 1945:466. Distr.: México e América Central.

intermedius Lacordaire, 1842:135. Tp. ? - (Rio de Janeiro). - Crotch, 1876:449. - Gemminger \& Harold, 1876:3693. - Kuhnt, 1909:76; 1911:50. - Blackwelder, 1945:466. Distr.: Brasil, região sudeste.

interstictus Gorham, 1888:50. Tp.: NHML exam. aut. - (Nicarágua, Chontales). - Kuhnt, 1909:76; 1911:50. - Blackwelder. 1945:466. Distr.: Nicarágua. interstitialis Kuhnt, 1910:239, fig. 8. Tp.: ZMHU exam. aut. - (Panamá, istmo Mataschin); 1911:50. - Blackwelder, 1945:466. Distr.: Panamá. 
jocosus Lacordaire, 1842:173. Tp.: MNHN exam. aut. - (Brasil). - Crotch, 1876:443. - Gemminger \& Harold, 1876:3693. - Kuhnt, 1909:75; 1911:50.

- Blackwelder, 1945:466. Distr.: Brasil, região sudeste.

laccophilinus Gorham, 1888:57, pl. 4, fig. 5. Tp.: NHML exam. aut. - (Panamá, Bugaba). - Kuhnt, 1909:71; 1911:50. - Blackwelder, 1945:466. Distr.: Panamá.

lacertosus Lacordaire, 1842:176. Tp. ? - (Caiena). - Crotch, 1876:446. - Gemminger \& Harold, 1876:3693. - Kuhnt, 1909:74; 1911:50. - Blackwelder, 1945:466. Distr.: Guiana Francesa.

laeviventris Crotch, 1876:454. Tp.: MCZC exam. aut. - (México). - Gemminger \& Harold, 1876:3693. - Gorham, 1888:51. - Kuhnt, 1909:76; 1911:50. Blackwelder, 1945:466. Distr.: México.

lepidus Lacordaire, 1842:178. Tp. ? - (Caiena). - Crotch, 1876:446. - Gemminger \& Harold, 1876:3693. - Kuhnt, 1909:72; 1911:50. - Deelder, 1942:88. Blackwelder, 1945:466. Distr.: Suriname. Guiana Francesa. Equador.

leprosus Lacordaire, 1842:160. Tp.: MCZC exam. aut. - (Caiena). - Crotch, 1876:439. - Gemminger \& Harold, 1876:3693. - Kuhnt, 1909:72; 1911:50. - Blackwelder, 1945:466. Distr.: Guiana Francesa.

lesueri Chevrolat, 1835, fasc. 8 (Erotylus). Tp.: MCZC exam. aut. - (México). Lacordaire, 1842:155 (Mycotretus). - Crotch, 1876:455. - Gemminger \& Harold, 1876:3693. - Gorham, 1888:59, pl. 3, fig. 20. - Kuhnt, 1909:73; 1911:50. - Deelder, 1942:88. - Blackwelder, 1945:466. Distr.: México. Belize. Guatemala.

limbatus Lacordaire, 1842:223 (Tritoma). Tp. ? - (Brasil). - Crotch, 1876:458 (Mycotretus). - Gemminger \& Harold, 1876:3693. - Kuhnt, 1909:74; 1911:50. - Blackwelder, 1945:466. Distr.: Brasil.

lissomoides Crotch, 1876:455. Tp.: MCZC exam. aut. - (Ega)[atualmente, Tefé]. - Gemminger \& Harold, 1876:3693. - Kuhnt, 1909:75; 1911:50. - Blackwelder, 1945:466. Distr.: Brasil, região amazônica.

lopesi Alvarenga, 1989:35-36, Tp.: MNRJ - (Brasil, Pará, Jacareacanga). Distr.: Brasil, região amazônica.

luizi Alvarenga, 1983:588. Tp.: CARJ - (Brasil, Amazonas, rio Javari, Estirão do Equador). Distr.: Brasil, região amazônica.

luteipes Lacordaire, 1842:189. Tp.: MCZC exam. aut. - (México). - Crotch, 1876:457. - Gemminger \& Harold, 1876:3693. - Gorham, 1888:61. Kuhnt, 1909:73; 1911:50. - Blackwelder, 1945:466. Distr.: México. Guatemala.

luteolus Gorham, 1888:58. Tp.: NHML - (Panamá, Volcán de Chiriquí). - Kuhnt, 1909:73; 1911:50. - Blackwelder, 1945:466. Distr.: Panamá.

maculatus Olivier, 1792:436 (Erotylus). Tp. ? - (Suriname); 1807:483, pl. 3, fig. 36. - Duponchel, 1825:176. - Lacordaire, 1842:192. - Crotch, 1876:438 
(Mycotretus). - Gemminger \& Harold, 1876:3693. - Gorham, 1888:49, pl. 4, fig. 1. - Kuhnt, 1909:76; 1911:50. - Curran, 1941:286-287. - Blackwelder, 1945:466. - Guérin, 1948:20. Distr.: Da América Central até o sul do Brasil, pelo litoral.

figuratus Lacordaire, 1842:159. - Chevrolat, 1843:79 (syn.).

magus Lacordaire, 1842:184. Tp. ? - (Brasil). - Crotch, 1876:456. - Gemminger \& Harold, 1876:3693. - Kuhnt, 1909:76; 1911:50. - Blackwelder, 1945: 466. Distr.: Brasil, região sudeste.

major Mader, 1955:477. Tp.: MGFM exam. aut. - (Paraguai, Villarica). Distr.: Paraguai.

marginicollis Lacordaire, 1842:159. Tp.: MCZC exam. aut. - (Brasil). - GuérinMéneville, 1855:610. - Crotch, 1876:438. - Gemminger \& Harold, 1876: 3693. - Kuhnt, 1909:76; 1911:51. - Deelder, 1942:88. - Blackwelder, 1945:466. - Guérin, 1948:20. Distr.: Brasil, regiões sudeste e sul.

melanophthalmus Duponchel, 1825:160, pl. 7, fig. 70 (Erotylus) Tp. ? - (Brasil). - Lacordaire, 1842:179 (Mycotretus). - Guérin-Méneville, 1855:610. Crotch, 1876:448. - Gemminger \& Harold, 1876:3693. - Kuhnt, 1909:72; 1911:51. - Blackwelder, 1945:466. - Guérin, 1948:20. Distr.: Brasil, região sudeste.

melanopterus Lacordaire, 1842:162. Tp.: MCZC exam. aut. - (Colômbia). Gemminger \& Harold, 1876:3693. - Kuhnt, 1909:71; 1911:51. - Blackwelder, 1945:466. Distr.: Colômbia.

xanthosomus Lacordaire, 1842:162. - Crotch, 1876:439 (syn.).

melanotus Gorham, 1888:66. Tp.: NHML - (Panamá, Chiriquí, La Caldera). Kuhnt, 1909:71; 1911:51. - Blackwelder, 1945:466. Distr.: Panamá.

miniatus Lacordaire, 1842:183. Tp. ? - (México). - Crotch, 1876:449. - Gemminger \& Harold, 1876:3693. - Gorham, 1888:65. - Kuhnt, 1909:73; 1911:51. - Blackwelder, 1945:466. Distr.: México. Guatemala.

minutus Duponchel, 1825:54, pl. 2, fig. 41 (Erotylus). Tp. ? - (Brasil). Lacordaire, 1842:154 (Mycotretus). - Crotch, 1876:455. - Gemminger \& Harold, 1876:3693. - Kuhnt, 1909:73; 1911:51. - Blackwelder, 1945:466.

- Guérin, 1948:20. Distr.: Brasil, região sudeste.

quadrinus Lacordaire, 1842:155. - Crotch, 1876:455 (syn.).

misellus Lacordaire, 1842:183. Tp. ? - (Caiena). - Crotch, 1876:447. - Gemminger \& Harold, 1876:3693. - Kuhnt, 1909:72; 1911:51. - Blackwelder, 1945: 466. - Guérin, 1948:20. Distr.: Colômbia. Guiana Francesa. Brasil, região sudeste.

monrosi Guérin, 1949b:589, fig. 1. Tp.: IMLT exam. aut. - (Argentina, Salta, Piletas). Distr.: Argentina.

multimaculatus Taschenberg, 1870:197. Tp. ? - (Colômbia). - Gemminger \& Harold, 1876:3693. - Kuhnt, 1909:74; 1911:51. - Blackwelder, 1945:467. - Mader, 1942d:174; 1951:216. Distr.: Colômbia. Equador. Peru. 
mutabilis Crotch, 1876:438. Tp.: MCZC exam. aut. - (Ega)[atualmente Tefé]. Gemminger \& Harold, 1876:3694. - Kuhnt, 1909:76; 1911:51. - Blackwelder, 1945:467. Distr.: Brasil, região amazônica.

mycetophagoides Crotch, 1876:457. Tp.: MCZC exam. aut. - (Pará). - Gemminger \& Harold, 1876:3694. - Kuhnt, 1909:71; 1911:51. - Blackwelder, 1945:467. Distr.: Brasil, região amazônica.

var. erythrocerus Crotch, 1876:457. Tp. ? - (Tapajós). - Gemminger \& Harold, 1876:3694. - Kuhnt, 1909:71; 1911:51. - Blackwelder, 1945:467. Distr.: Brasil, região amazônica.

mycetophiloides Crotch, 1876:458. Tp.: MCZC exam. aut. - (Ega)[atualmente Tefé]. - Gemminger \& Harold, 1876:3694. - Kuhnt, 1909:74; 1911:51. Blackwelder, 1945:467. Distr.: Brasil, região amazônica.

var. careus Crotch, 1876:458. Tp.: MCZC exam. aut. - [Localidade não citada]. - Kuhnt, 1909:74; 1911:51. - Blackwelder, 1945:467. Distr.: Brasil, região amazônica.

nigricollis Gorham, 1888:70. Tp.: NHML - (México, Toxpam). - Kuhnt, 1909:71; 1911:51. - Blackwelder, 1945:467. Distr.: México.

nigripes Gorham, 1888:64. Tp.: NHML - (Guatemala, Cerro Zunil). - Kuhnt, 1909:73; 1911:51. - Blackwelder, 1945:467. Distr.: Guatemala.

nigrivittis Lacordaire, 1842:157. Tp. ? - (Caiena). - Crotch, 1876:437. Gemminger \& Harold, 1876:3694. - Kuhnt, 1909:75; 1911:51. - Blackwelder, 1945:467. Distr.: Guiana Francesa.

nigromaculatus Kuhnt, 1909:27 (Brachysphaenus). Tp.: ZMHU exam. aut. (México); 1911:17. - Blackwelder, 1945:459. - Delkeskamp, 1939:27 (Mycotretus). Distr.: México.

nigromanicatus Boyle, 1954b:48. Tp.: ANSP - (U.S.A., Arizona, Cochise, Chiricahua mts.); 1956:138, figs 139, 140. Distr.: U.S.A. e México.

nigropunctatus Duponchel, 1825:51, pl. 2, fig. 34 (Erotylus). Tp. ? (Brasil). Lacordaire, 1842:142 (Mycotretus). - Crotch, 1876:450. - Gemminger \& Harold, 1876:3694. - Gorham, 1887:48. - Kuhnt, 1909:75; 1911:51. Bruch, 1914:384. - Deelder, 1942:88. - Blackwelder, 1945:467. - Guérin, 1948:20. Distr.: Costa Rica. Brasil, regiões sudeste e sul. Argentina.

puncticollis Duponchel, 1825:54, pl. 2, fig. 43 (Erotylus). - Lacordaire, 1842:142 (syn.).

nigroterminatus Lacordaire, 1842:180. Tp. ? - (Colômbia). - Crotch, 1876:448. - Gemminger \& Harold, 1876:3694. - Kuhnt, 1909:74; 1911:51. - Blackwelder, 1945:467. - Guérin, 1948:20. Distr.: Colômbia. Brasil, região sudeste.

nitescens Crotch, 1876:445. Tp.: MCZC exam. aut. - (Ega)[atualmente Tefé]. Gemminger \& Harold, 1876:3694. - Gorham, 1888:52. - Kuhnt, 1909:72; 1911:51. - Blackwelder, 1945:467. Distr.: México. Guatemala. Panamá. Brasil, região amazônica. 
normalis Gorham, 1888:51. Tp.: NHML - (México, Córdova). - Kuhnt, 1909:72; 1911:51. - Blackwelder, 1945:467. Distr.: México.

noterinus Gorham, 1888:65. Tp.: NHML exam. aut. - (Panamá, Volcán de Chiriquí). - Kuhnt, 1909:72; 1911:51. - Deelder, 1942:88. - Blackwelder, 1945:467. Distr.: Panamá.

nubifer Casey, 1916:158. Tp.: NMNH - (Guatemala). - Blackwelder, 1945:467. Distr.: Guatemala.

nugator Lacordaire, 1842:174. Tp. ? - (Caiena). - Crotch, 1876:445. - Gemminger \& Harold, 1876:3694. - Kuhnt, 1909:75; 1911:51. - Blackwelder, 1945: 467. Distr.: Guiana Francesa. Brasil, região amazônica.

oculatus Alvarenga, 1983:585. Tp.: CARJ - (Brasil, Pará, Tucuruí). Distr.: Brasil, região Amazônica.

opalescens Crotch, 1876:444. Tp.: MCZC exam. aut. - (Santarém). - Gemminger \& Harold, 1876:3694. - Kuhnt, 1909:76; 1911:51. - Blackwelder, 1945: 467. - Delkeskamp, 1957:99. Distr.: Bolívia. Brasil, região amazônica.

opalizans Mader, 1942d:175, 200. Tp.: DEIB - (Peru, Chanchamayo); 1951:223.

Distr.: Peru.

ab. posticenigrum Mader, 1942d:175, 200. Tp. ? - (Peru); 1951:223. Distr.: Peru.

oppositipunctum Gorham, 1888:69, pl. 4, fig. 6. Tp.: NHML exam. aut. (Guatemala, San Gerónimo). - Kuhnt, 1909:73; 1911:51. - Blackwelder. 1945:467. Distr.: Guatemala.

ornatus Duponchel, 1825:49, pl. 2, fig. 31 (Erotylus). Tp. ? - (Brasil). Lacordaire, 1842:137 (Mycotretus). - Crotch, 1876:449. - Gemminger \& Harold, 1876:3694. - Gorham, 1888:47, pl. 3, FIG. 8; 1898:253. - Kuhnt, 1909:76; 1911:51. - Deelder, 1942:88, figs 4, a e b. - Mader, 1942d:175; 1951:220-221. - Blackwelder, 1945:467. Guérin, 1948:20. - Delkeskamp, 1957:99. Distr.: Da América Central até o sul do Brasil.

var. godarti Lacordaire, 1842:146. Tp ? - (Colômbia). - Crotch, 1876:450. Gemminger \& Harold, 1876:3693. - Kuhnt, 1909:76; 1911:52. Deelder, 1942:88, fig. 4, d. - Mader, 1942d:175; 1951:218, 221. Blackwelder, 1945:467. Distr.: Colômbia.

var. maculosus Duponchel, 1825:52, pl. 2, fig. 36 (Erotylus). Tp. ? - (Brasil). - Lacordaire, 1842:140 (Mycotretus). - Crotch, 1876:449. - Gemminger \& Harold, 1876:3693. - Kuhnt, 1909:76; 1910:238; 1911:52. - Mader, 1942d:175; 1951:221. - Blackwelder, 1945:467. Distr.: Venezuela. Brasil, região sudeste.

var. melanostictus Lacordaire, 1842:139. Tp. ? - (Colômbia). - Crotch, 1876:450. - Gemminger \& Harold, 1876:3693. - Kuhnt, 1909:76; 1911:52. - Mader, 1942d:175; 1951:221. - Blackwelder, 1945:467.

Guérin, 1948:20. Distr.: Colômbia. Brasil, região central.

var. posticus Lacordaire, 1842:147. Tp. ? - (Colômbia). - Crotch, 1876:450. 
- Gemminger \& Harold, 1876:3693. - Kuhnt, 1909:76; 1911:52. Mader, 1942d:175; 1951:218, 221. - Blackwelder, 1945:467. Distr.: Colômbia.

var. terminalis Lacordaire, 1842:134. Tp. ? - (Rio de Janeiro). - Crotch, 1876:449. Gemminger \& Harold, 1876:3695. - Kuhnt, 1909:76; 1911: 52. - Deelder, 1942:88, fig. 4, c. - Mader, 1942d:175; 1951:221, 223.

- Blackwelder, 1945:467. Distr.: Brasil, região sudeste.

var. partitialis Deelder, 1942:88. fig. 4, e. Tp.: RNHL - (Colômbia, Cauca). - Delkeskamp, 1957:113 (= M. partitialis Mader, 1940).

pallidior Crotch, 1876:428 (Ischyrus). Tp.: MCZC exam. aut. - (México). Gemminger \& Harold, 1876:3691. - Gorham, 1888:52, pl. 2, fig. 18 (Mycotretus). - Kuhnt, 1909:75; 1911:52. - Blackwelder, 1945:467. Distr.: México e América Central.

nigrotinctus Crotch, 1876:454. - Gorham, 1888:52 (syn.).

palmiphilus Lacordaire, 1842:165. Tp. ? - (Caiena). - Crotch, 1876:439. - Gemminger \& Harold, 1876:3694. - Kuhnt, 1909:71; 1911:52. - Blackwelder, 1945:467. Distr.: Guiana Francesa. Brasil, região amazônica.

panamanus Gorham, 1888:54, pl. 3, fig. 14. Tp.: NHML exam. aut. - (Panamá, Bugaba). - Kuhnt, 1909:75; 1911:52. - Blackwelder, 1945:467. Distr.: Panamá.

parallelus Crotch, 1876:453. Tp.: MCZC exam. aut. - (Ega) [atualmente Tefé]. - Gemminger \& Harold, 1876:3694. - Kuhnt, 1909:73; 1911:52. - Blackwelder, 1945:467. Distr.: Brasil, região amazônica.

partitialis Mader, 1940:12. Tp. ? - (Colômbia, Cauca). - Blackwelder, 1945:467.

- Delkeskamp, 1957:113. Distr.: Colômbia.

partitus Lacordaire, 1842:176. Tp. ? - Colômbia). - Crotch, 1876:446. - Gemminger \& Harold, 1876:3694. - Kuhnt, 1909:75; 1911:52. - Blackwelder, 1945:467. Distr.: Colômbia.

pebasensis Crotch, 1876:439. Tp.: MCZC exam. aut. - (Pebas). - Gemminger \& Harold, 1876:3692. - Kuhnt, 1909:76; 1911:52. - Mader, 1942d:175; 1951:223. - Blackwelder, 1945:467. Distr.: Equador.

pecari Lacordaire, 1842:167. Tp. ? - (Colômbia, Valencia, Maracaibo). - Crotch, 1876:440. - Gemminger \& Harold, 1876:3694. - Gorham, 1888:55, pl. 3, figs 18, 19. - Kuhnt, 1909:71; 1911:52. - Deelder, 1942:89. - Blackwelder, 1945:467. - Guérin, 1948:20. Distr.: Nicarágua. Panamá. Colômbia. Brasil, região amazônica.

pelliciens Kirsch, 1876:100. Tp.: SMTD exam. aut. - (Peru). - Gemminger \& Harold, 1876:3694. - Kuhnt, 1909:76; 1911:52. - Mader, 1942d:174; 1951:219. - Blackwelder, 1945:467. Distr.: Peru.

peruae Crotch, 1876:446. Tp.: MCZC exam. aut. - (Peru). - Gemminger \& Harold, 1876:3694. - Kuhnt, 1909:73; 1911:52. - Mader, 1942d:172; 1951:213. - Blackwelder, 1945:467. Distr.: Peru. 
pictopiceus Gorham, 1888:56. Tp.: NHML exam. aut. - (Panamá, Bugaba). Kuhnt, 1909:71; 1911:52. - Blackwelder, 1945:467. Distr.: Panamá.

planus Gorham, 1888:66. Tp.: NHML - (Panamá, Bugaba). - Kuhnt, 1909:72; 1911:52. - Blackwelder, 1945:467. Distr.: Panamá.

polyophthalmus Lacordaire, 1842:163. Tp. ? - (Caiena). - Crotch, 1876:441. Gemminger \& Harold, 1876:3694. - Kuhnt, 1909:74; 1911:52. - Blackwelder, 1945:467. Distr.: Guiana Francesa. Venezuela.

prioteloides Mader, 1942d:174, 196. Tp.: MGFM exam. aut. -(Peru, Callanga); 1951:217. Distr.: Peru. Bolívia.

psittacus Lacordaire, 1842:167. Tp. ? - (México). [Erro na localidade]. - Crotch, 1876:440. - Gemminger \& Harold, 1876:3694. - Gorham, 1888:54. Kuhnt, 1909:72; 1911:52. - Blackwelder, 1945:467. Distr.: Brasil, região sudeste.

psylloboroides Crotch, 1876:443. Tp.: MCZC exam. aut. - (Ega) [atualmente Tefél. - Gemminger \& Harold, 1876:3694. - Kuhnt, 1909:72; 1911:52. Blackwelder, 1945:467. Distr.: Brasil, região amazônica.

pulchellus Lacordaire, 1842:177. Tp. ? - (Brasil). - Crotch, 1876:446. - Gemminger \& Harold, 1876:3694. - Kuhnt, 1909:71; 1911:52. - Blackwelder, 1945:467. Distr.: Brasil, região sudeste.

puncticeps Kirsch, 1865:97. Tp.: SMTD exam. aut. - (Colômbia, Bogotá). Crotch, 1876:454. Gemminger \& Harold, 1876:3694. - Kuhnt, 1909:76; 1911:52. - Deelder, 1942:89. - Blackwelder, 1945:467. Distr.: Colômbia. pusillus Lacordaire, 1842:188. Tp. ? -(Caiena). - Crotch, 1876:456. - Gemminger \& Harold, 1876:3694. - Kuhnt, 1909:72; 1911:52. - Blackwelder, 1945: 467. Distr.: Guiana Francesa.

pygmaeus Lacordaire, 1842:156. Tp. ? - (Caiena). - Crotch, 1876:455. - Gemminger \& Harold, 1876:3694. - Gorham, 1888:62. - Kuhnt, 1909:72; 1911:52. - Bruch, 1914:384. - Curran, 1941:286. - Mader, 1942d:172; 1951:211. - Blackwelder, 1945:467.- Guérin, 1948:20. Distr.: Do México até a Argentina.

quadrioculatus Alvarenga, 1983:585. Tp.: CARJ - (Brasil, Pará, Tucuruí). Distr.: Brasil, região amazônica.

quadripunctatus Crotch, 1876:450. Tp.: MCZC exam. aut. - (Norte do Peru). Gemminger \& Harold, 1876:3694. - Kuhnt, 1909:76; 1911:52. - Mader. 1942d:174; 1951:219, 223. - Blackwelder, 1945:467. Distr.: Colômbia. Equador. Peru.

quadristriolatus Kuhnt, 1910:242. Tp.: ZMHU exam. aut. - (Peru, Chanchamayo); 1911:52. - Mader, 1942d:174: 1951:217. - Blackwelder, 1945:467. Distr.: Peru.

quattuordecimguttatus Lacordaire, 1842:163. Tp.: MCZC exam. aut. - (Colômbia). - Crotch, 1876:439. - Gemminger \& Harold, 1876:3694. - Kuhnt, 
1909:71; 1911:53. - Deelder, 1942:89. - Blackwelder, 1945:467. Distr.:

Colômbia. Brasil, região amazônica.

var. conjunctus Crotch, 1876:440. Tp.: MCZC exam. aut. - [Localidade não citada]. - Gemminger \& Harold, 1876:3694. - Kuhnt, 1909:71; 1911: 53. - Blackwelder, 1945:467. Distr.: Colômbia.

rastratus Crotch, 1876:445. Tp.: MCZC exam. aut. - (São Paulo)[atualmente São

Paulo de Olivença]. - Gemminger \& Harold, 1876:3694. - Kuhnt, 1909:72;

1911:53. - Blackwelder, 1945:467. Distr.: Brasil, região amazônica.

reticulatus Crotch, 1876:443. Tp.: MCZC exam. aut. - (Ega) [atualmente Tefé].

- Gemminger \& Harold, 1876:3694. - Kuhnt, 1909:71; 1911:53. - Black-

welder, 1945:467. Distr.: Brasil, região amazônica.

rhodosomus Lacordaire, 1842:166. Tp.: MCZC exam. aut. - (Brasil). - Crotch, 1876:444. - Gemminger \& Harold, 1876:3694. - Kuhnt, 1909:72; 1911:53.

- Blackwelder, 1945:467. - Guérin, 1948:20. Distr.: Brasil, região sudeste.

rubidus Gorham, 1888:62. Tp.: NHML - (Guatemala, Chacoj, Panzos). - Kuhnt, 1909:73; 1911:53. - Blackwelder, 1945:467. Distr.: Guatemala.

rufilabris Lacordaire, 1842:222 (Tritoma). Tp. ? - (Brasil). - Crotch, 1876:458 (Mycotretus). - Gemminger \& Harold, 1876:3694. - Kuhnt, 1909:70; 1911:53. - Blackwelder, 1945:467. Distr.: Brasil.

rufipennis Gorham, 1888:69. Tp.: NHML - (México, Cerro de Pluma). - Kuhnt, 1909:73; 1911:53. - Blackwelder, 1945:467. Distr.: México.

sallei Crotch, 1876:452. Tp.: MCZC exam. aut. - (México). - Gemminger \& Harold, 1876:3695. - Gorham, 1888:53, pl. 4, fig. 2. - Kuhnt, 1909:75; 1911:53. - Blackwelder, 1945:467. Distr.: México.

sandicatus Gorham, 1888:54. Tp.: NHML exam. aut. - (Guatemala, Puruba, San Gerónimo). - Kuhnt, 1909:72; 1911:53. - Blackwelder, 1945:467. Distr.: Guatemala.

sanguineus Duponchel, 1825:54, pl. 2, fig. 42 (Erotylus). Tp. ? - (Brasil). Lacordaire, 1842:187 (Mycotretus). - Crotch, 1876:456. - Gemminger \& Harold, 1876:3695. - Kuhnt, 1909:72; 1911:53. - Blackwelder, 1945:467. - Guérin, 1948:20. Distr.: Colômbia. Brasil, região sudeste.

sanguinosus Crotch, 1876:458. Tp.: MCZC exam. aut. - (Colômbia). - Gemminger \& Harold, 1876:3695. - Gorham, 1888:68. - Kuhnt, 1909:72; 1911:53. - Blackwelder, 1945:467. - Distr.: Costa Rica. Panamá. Colômbia.

sannio Lacordaire, 1842:169. Tp. ? - (Brasil). - Crotch, 1876:441. - Gemminger \& Harold, 1876:3695. - Kuhnt, 1909:75; 1911:53. - Blackwelder, 1945: 467. Distr.: Brasil.

savignyi Lacordaire, 1842:156. Tp. ? - (Colômbia). - Crotch, 1873b:145; 1876: 455. - Gemminger \& Harold, 1876:3695. - Gorham, 1888:60. - Kuhnt, 1909:72; 1911:53. - Deelder, 1942:89. - Mader, 1942d:172; 1951:212. Blackwelder, 1945:467. - Guérin, 1948:20. Distr.: Do México até o Brasil. 
scalaris Lacordaire, 1842:168. Tp. ? - (Colômbia). - Crotch, 1876:440. Gemminger \& Harold, 1876:3695. - Kuhnt, 1909:71; 1911:53. - Blackwelder, 1945:467. Distr.: Colômbia.

scitulus Lacordaire, 1842:154. Tp.: MCZC exam. aut. - (Rio de Janeiro). - Crotch, 1876:454. - Gemminger \& Harold, 1876:3695. - Gorham, 1888:49; 1898:253. - Kuhnt, 1909:75; 1911:53. - Mader, 1942d:174; 1951:219, 222. - Blackwelder, 1945:467. - Delkeskamp, 1957:99. Distr.: Do México até o sul do Brasil.

sedecimguttatus Guérin-Méneville, 1844:310 (Ischyrus). Tp. ? - (Colômbia). Crotch, 1876:440 (Mycotretus). - Gemminger \& Harold, 1876:3695. Kuhnt, 1909:71; 1911:53. - Blackwelder, 1945:467. Distr.: Colômbia.

seminiger Harold, 1876:174. - Gemminger \& Harold, 1876:3695. - Kuhnt, 1909:71; 1911:53. - Blackwelder, 1945:467. Distr.: Brasil, região amazônica.

dimidiatus; Crotch, 1876, nec Taschenberg, 1870. - Crotch, 1876:444. Tp. ? - (Ega). - Harold, 1876:174 (nom.praeoc.).

separandus Crotch, 1876:442. Tp.: MCZC exam. aut. - (Ega)[atualmente Tefé]. - Gemminger \& Harold, 1876:3695. - Kuhnt, 1909:76; 1911:53. - Blackwelder, 1945:467. Distr.: Brasil, região amazônica.

sericeonitens Crotch, 1876:445. Tp.: MCZC exam. aut. - (Ega)[atualmente Tefé]. - Gemminger \& Harold, 1876:3695. - Kuhnt, 1909:76; 1911:53. - Mader, 1942d:175; 1951:223. - Blackwelder, 1945:467. - Delkeskamp, 1957:99.

Distr.: Equador. Peru. Bolívia. Brasil, região amazônica.

var. monticola Crotch, 1876:445. Tp. ? - (Equador). - Kuhnt, 1909:76; 1911:53. - Blackwelder, 1945:467. - Mader, 1951:223. Distr.: Equador.

sexlineatus Kuhnt, 1910:241, fig. 10. Tp.: ZMHU exam. aut. - (Brasil); 1911:53.

- Blackwelder, 1945:467. - Guérin, 1948:20. Distr.: Brasil, Paraná.

sexoculatus chaparensis Delkeskamp, 1957:98, 111, fig. 9. Tp.: ZSBS - (Bolívia, Chapare, alto rio Chipiriri). Distr.: Bolívia.

sexoculatus sexoculatus Lacordaire, 1842:165. Tp.: MCZC exam. aut. - (Colômbia). - Crotch, 1876:439. - Gemminger \& Harold, 1876:3695. - Kuhnt, 1909:74; 1911:53. - Blackwelder, 1945:467. Distr.: Colômbia.

sexpunctatus Gorham, 1888:50, pl. 3, fig. 12. Tp.: NHML - (Panamá, Bugaba).

- Kuhnt, 1909:76; 1911:53. - Blackwelder, 1945:467. Distr.: Panamá.

signatellus Crotch, 1876:443. Tp.: MCZC exam. aut. - (Ega) [atualmente Tefé]. - Gemminger \& Harold, 1876:3695. - Kuhnt, 1909:76; 1911:53. - Blackwelder, 1945:467. Distr.: Brasil, região amazônica.

var. imperfectus Crotch, 1876:443. Tp.: MCZC exam. aut. - (Pará). - Kuhnt, 1909:76; 1911:53. - Blackwelder, 1945:467. Distr.; Brasil, região amazônica.

singularis Lacordaire, 1842: 148. Tp.: MCZC exam. aut. - (Colômbia). - Crotch, 
1876:451. - Gemminger \& Harold, 1876:3695. - Kuhnt, 1909:74; 1911:53. - Blackwelder, 1945:467. Distr.: Colômbia.

sobrinus Guérin-Méneville, 1841 b: 154 (Brachymerus). Tp.: MCZC exam. aut. (Brasil). - Lacordaire, 1842:186 (Mycotretus). - Crotch, 1876:456. Gemminger \& Harold, 1876:3695. - Gorham, 1888:60. - Kuhnt, 1909:72; 1911:53. - Deelder, 1942:90. - Blackwelder, 1945:467. - Guérin, 1948:20. Distr.: Brasil.

silaceus Lacordaire, 1842:187. - Gorham, 1888:60 (syn.).

spadiceus Gorham, 1888:53, pl. 3, figs 16, 17. Tp.: NHML exam. aut. (Nicarágua, Chontales). Kuhnt, 1909:74, pl. 3, fíg. 8; 1910:239; 191 1:54. - Blackwelder, 1945:467. Distr.: América Central. Colômbia.

sticticollis Lacordaire, 1842:174. Tp. ? - (Caiena). - Crotch, 1876:446. - Gemminger \& Harold, 1876:3695. - Kuhnt, 1909:73; 1911:54. - Blackwelder, 1945:467. Distr.: Guiana Francesa.

stillatus Kuhnt, 1910:239. Tp.: ZMHU exam. aut. - (Venezuela, Caracas); 1911:54. - Blackwelder, 1945:467. Distr.: Venezuela.

stramineus Gorham, 1888:58. Tp.: NHML - (Panamá, Bugaba). - Kuhnt, 1909:71; 1911:54. - Blackwelder, 1945:467. Distr.: Panamá.

succinctus Crotch, 1876:452. Tp.: MCZC exam. aut. - (Colômbia). - Gemminger \& Harold, 1876:3695. - Kuhnt, 1909:74; 1911:54. - Deelder, 1942:90. Blackwelder, 1945:467. Distr.: Colômbia.

suturalis Kirsch, 1876:99. Tp.: SMTD exam. aut. - (Peru). - Gemminger \& Harold, 1876:3695. - Kuhnt, 1909:72; 1911:54. - Mader, 1942d:172; 1951:213. - Blackwelder, 1945:467. Distr.: Peru. Bolívia.

ternotatus Gorham, 1888:51, pl. 3, fig. 13. Tp.: NHML exam. aut. - (México, Jalapa). - Kuhnt, 1909:76; 1911:54. - Blackwelder, 1945:467. Distr.: México.

tesserarius Lacordaire, 1842:191. Tp. ? - (Colômbia). - Crotch, 1876:458. Gemminger \& Harold, 1876:3695. - Kuhnt, 1909:75; 1911:54. - Blackwelder, 1945:467. Distr.: Colômbia.

thoracicus Kuhnt, 1910:242, fig. 11. Tp.: ZMHU exam. aut. - (Peru, Chanchamayo); 1911:54. - Mader, 1942d:175; 1951:222. - Blackwelder, 1945:467. Distr.: Peru.

tibialis Gorham, 1888:56. Tp.: NHML exam. aut. - (Nicarágua, Chontales). Kuhnt, 1909:72; 1911:54. - Blackwelder, 1945:467. Distr.: Nicarágua.

tigratus Lacordaire, 1842:150. Tp. ? - (Colômbia). - Crotch, 1876:451. - Gemminger \& Harold, 1876:3695. - Kuhnt, 1909:74; 1911:54. - Blackwelder, 1945:467. - Mader, 1951:217. Distr.: Colômbia.

ab. nigrocinctus Lacordaire, 1842:151. Tp. ? - (Colômbia). - Crotch, 1876: 451. - Kuhnt, 1909:74; 1911:54. - Blackwelder, 1945:467. - Mader, 1951:217. Distr.: Colômbia. 
tigrinoides Mader, 1942d:174, 196. Tp.: DEIB (Peru, Ocobamba); 1951:216. Distr.: Peru.

tigrinus Olivier, 1792:437 (Erotylus). Tp. ? - (Suriname); 1807:485, pl. 3, fig. 40. - Duponchel, 1825:51, pl. 2, fig. 35. - Lacordaire, 1842:145 (Mycotretus). - Erichson, 1847:180. - Guérin-Méneville, 1855:610. - Fauvel, 1860:326. - Crotch, 1876:451. - Gemminger \& Harold, 1876:3695. Kirsch, 1876:101. - Kuhnt, 1909:74; 1911:54. - Deelder, 1942:90. Mader, 1942d:173; 1951:216. - Blackwelder, 1945:468. - Guérin, 1948: 20. - Delkeskamp, 1957:99. Distr.: Equador. Peru. Suriname. Guiana Francesa. Bolívia. Brasil.

conspersus Germar, 1824:614 (Erotylus). - Lacordaire, 1842:145 (syn.).

leopardus Gorham, 1888:49. - Kuhnt, 1911:54 (syn.).

var. pardalis Crotch, 1876:451. Tp. ? - (Equador). - Gemminger \& Harold, 1876:3695. - Kuhnt, 1909:74; 1911:54. - Mader, 1942d:173; 1951:216.

- Blackwelder, 1945:468. Distr.: Peru. Equador. Brasil, região amazônica.

tigripennis Mader, 1942d:174, 197. Tp.: DEIB (Equador, Santa Inez); 1951:217. Distr.: Equador.

trabeatus Lacordaire, 1842:148. Tp. ? - (Colômbia). - Crotch, 1876:451. Gemminger \& Harold, 1876:3695. - Kuhnt, 1909:74; 1911:54. - Deelder, 1942:90. - Mader, 1951:216. - Blackwelder, 1945:468. Distr.: Colômbia.

tricolor Crotch, 1876:444. Tp.: MCZC exam. aut. - (Ega) [atualmente Tefé]. Gemminger \& Harold, 1876:3695. - Kuhnt, 1909:71; 1911:54. - Mader, 1942d:172; 1951:212. - Blackwelder, 1945:468. Distr.: Peru. Brasil, região amazônica.

trifasciatus Guérin, 1956:63, fig. 26. Tp.: CDSP exam. aut. - (Brasil, Santa Catarina, Nova Teutônia). Distr.: Brasil, região sudeste e sul.

tucuruiensis Alvarenga, 1983:586. Tp.: CARJ - (Brasil, Pará, Tucuruí). Distr.: Brasil, região amazônica.

ab. luteobifasciatus Mader, 1942d:174; 1951:223. Distr.: Peru.

unicolor Fauvel, 1860:326. Tp. ? - (Caiena). - Gemminger \& Harold, 1876:3695. - Kuhnt, 1911:54. - Blackwelder, 1945:468. Distr.: Guiana Francesa.

vilis Lacordaire, 1842:175. Tp. ? - (Caiena). - Crotch, 1876:446. - Gemminger \& Harold, 1876:3695. - Kuhnt, 1909:73; 1911:54. - Blackwelder, 1945: 468. Distr.: Guiana Francesa.

virgatus Kuhnt, 1910:243. Tp.: ZMHU exam. aut. - (Brasil); 1911:54. - Blackwelder, 1945:468. - Guérin, 1948:20. Distr.: Brasil, regiões sudeste e sul.

vittatus Gorham, 1888:57, pl. 3, fig. 21. Tp.: NHML exam. aut. - (Guatemala, Pantaleon, Zapote); 1898:253. - Kuhnt, 1909:76; 1911:54. - Blackwelder, 1945:468. Distr.: México. Guatemala.

xanthomelas Crotch, 1876:458. Tp.: MCZC exam. aut. - (Pará). - Gemminger \& 
Harold, 1876:3695. - Kuhnt, 1909:74; 1911:54. - Blackwelder, 1945:468.

Distr.: Brasil, região amazônica.

ziczac serenus Delkeskamp, 1957:99, 110, fig. 8. Tp.: ZSBS - (Bolívia, rio Yacuma, Santa Roza). Distr.: Bolívia.

ziczac ziczac Kuhnt, 1910:241. Tp.: ZMHU exam. aut. - (Colômbia, Muzo); 1911:54. - Blackwelder, 1945:468. Distr.: Colômbia.

zischkai Delkeskamp, 1957:98, 112, fig. 10. Tp.: ZSBS exam. aut. - (Bolívia, Chapare, alto rio Chipiriri). Distr.: Bolívia.

\section{Mycomystes Gorham, 1888}

Mycomystes Gorham, 1888:71 (Tgen. Mycomystes ferrugineus Gorham, 1888:71, monotipia). - Kuhnt, 1909:58, 77. Deelder, 1942:54. Alvarenga, 1965:86.

ferrugineus Gorham, 1888:71, pl. 4, fig. 8. Tp.: NHML exam. aut. - (México, Cerro de Plumas). - Kuhnt, 1909:77; 1911:54. - Blackwelder, 1945:468. Distr.: México.

\section{Tritomapara Alvarenga, 1970}

Tritomapara Alvarenga, 1970:45 (Tgen.: Paratritoma dimidiata Gorham, 1888: 72, desig. orig.).

atricaudata Kuhnt, 1910:246 (Paratritoma). Tp.: ZMHU - (Venezuela, Aragua); 1911:55. - Blackwelder, 1945:468. Distr.: Venezuela.

brasiliensis Guérin, 1946a:275, fig. 2 (Paratritoma). Tp.: CDSP exam. aut. (Brasil, Est. S. Paulo, Cantareira). Distr.: Brasil, região sudeste.

bruchi Kuhnt, 1910:245-246, fig. 12 (Paratritoma). Tp. ? - (Argentina, Santa Fé, Chaco); 1911:55. - Bruch, 1914:384. - Blackwelder, 1945:468. Distr.: Argentina.

caduca Gorham, 1888:73, pl. 4, fig. 12 (Paratritoma). Tp.: NHML - (Guatemala, Cerro Zunil). - Kuhnt, 1909:77; 1910:247; 1911:55. - Blackwelder, 1945:468. Distr.: Guatemala.

dimidiata Gorham, 1888:72 (Paratritoma). Tp.: NHML - (México, Cerro de Plumas). - Kuhnt, 1909:77; 1910:246; 1911:55. - Blackwelder, 1945:468.

Distr.: México.

melanoderes Kuhnt, 1910:245 (Paratritoma). Tp ? - (Colômbia); 1911:55. Blackwelder, 1945:468. Distr.: Colômbia.

triplacoides Crotch, 1876:447 (Mycotretus). Tp.: MCZC exam. aut. - (Santarém).

- Gorham, 1888:72 (Paratritoma). -Kuhnt, 1909:77; 1910:246; 1911:55.

- Blackwelder, 1945:468. Distr.: Brasil, região amazônica.

vivida Gorham, 1888:73 (Paratritoma). Tp.: NHML - (Guatemala, Cerro Zunil). 
- Kuhnt, 1909:77; 1910:246; 1911:55. - Blackwelder, 1945:468. Distr.: Guatemala.

\section{Tritoma Fabricius, 1775}

Tritoma Fabricius, 1775:68 (Tgen. Tritoma bipustulata Fabricius, 1775:68, seg. Latreille, 1810:239). - Lacordaire, 1842:31, 218. - Chapuis, 1876:31, 43. Gorham, 1888:79. - Kuhnt, 1909:56, 77. - Deelder, 1942:53. - Boyle, 1956:96, 117. - Alvarenga, 1965:90. - Delkeskamp, 1981:34.

Cyrtotriplax Crotch, 1873:189. - Gorham, 1896:58. - Delkeskamp, 1981:34 (syn.).

dorsalis Gorham, 1888:80, pl. 4, fig. 20. Tp.: NHML - (Panamá, Bugaba). Kuhnt, 1909:79; 1911:56. - Blackwelder, 1945:468. Distr.: Panamá.

\section{Haematochiton Gorham, 1888}

Haematochiton Gorham, 1888:82 (Tgen. Haematochiton elateroides Gorham, 1888:81, monotipia). - Kuhnt, 1909:56, 81. - Deelder, 1942:52. - Boyle, 1956:97, 138. - Alvarenga, 1965:85. - Johnson, 1967:15.

Scaeother Gorham, 1888:81. - Kuhnt, 1909:56, 81. - Deelder, 1942:52. - Boyle, 1956: 138 (syn.).

carbonarius Gorham, 1888:82, pl. 5, fig. 5 (Scaeother). Tp.: NHML exam. aut. - (México, Toluca). - Kuhnt, 1909:81; 1911:58. - Blackwelder, 1945:468. - Boyle, 1956:139, fig. 137-138 (Haematochiton). - Johson, 1967:17, 21.

Distr.: U.S.A. e México.

opacus Schaeffer, 1915:236 (Scaeother). - Boyle, 1956:140 (syn.).

cruentipennis Lacordaire, 1842:205 (Triplax). Tp.: MNHN - (Brasil). - Crotch, 1876:467. - Gemminger \& Harold, 1876:3697. - Kuhnt, 1909:86; 1911:60.

- Blackwelder, 1945:468. - Johnson, 1967:18-19, fig. 8 (Haematochiton). Distr.: Brasil.

elateroides Gorham, 1888:81, pl. 5, fig. 4. Tp.: NHML exam. aut. - (México, Durango). - Kuhnt, 1909:81; 1911:58. - Leng, 1920:201. - Blackwelder, 1945:468. - Boyle, 1956:139, fig. 133-136. - Johnson, 1967:17. Distr.: U.S.A. e México.

bisculptum Casey, 1916:168. - Boyle, 1956:139 (syn.).

maderi Delkeskamp, 1957:114, fig. 11 (Triplax). Tp.: ZMHU exam. aut. - (Brasil,

Santa Catarina, Nova Teutônia). - Johson, 1967:17, 20, fig. 10 (Haematochiton). Distr.: Brasil, regiões sudeste e sul. 


\section{Triplax Herbst, 1793}

Triplax Herbst, 1793: 146 (Tgen.: Silpha russica Linnaeus, 1758:360, seg. Curtis, 1838:706). - Lacordaire, 1842:31, 202. - Crotch, 1876:463. - Chapuis, 1876:31, 40. - Gorham, 1888:78. - Kuhnt, 1909:57, 81. - Guérin, 1952:180. - Boyle, 1956:96. - Alvarenga, 1965:90. - Johnson, 1965:1.

Paratritoma Gorham, 1888:71 (Tgen.: Paratritoma divisa Gorham, 1888:72, seg. Alvarenga 1965:88). - Kuhnt, 1909:58, 77. - Deelder, 1942:54. - Alvarenga, 1965:88). - Johnson, 1967:8 (syn.).

alvarengai Johnson, 1967:5-6, fig. 1. Tp.: FMNH - (Costa Rica, Ciruclas). Distr.: México e América Central.

azureipennis Guérin, 1952:182, fig. 4. Tp. ? - (Chile, Paulsen). Distr.: Chile. bicolor Guérin, 1952:182, fig. 5. Tp. ? - (Chile, Paulsen). Distr.: Chile. divisa Gorham, 1888:72, pl. 4, fig. 9 (Paratritoma). Tp.: NHML - (México, Esperanza, Jalapa, Cerro de Plumas). - Kuhnt, 1909:77; 1910:247; 1911: 55. - Blackwelder, 1945:468. - Johnson, 1967:6, 8, fig. 2 (Triplax). Distr.: México e América Central.

errans Boyle, 1956:101, 110, fig. 99. Tp.: UK - (U.S.A., Texas, Cameron, Brownsville). - Johnson, 1967:5, 9, fig. 3. Distr.: U.S.A. e México.

flavicollis Lacordaire, 1842:218. Tp. ? - (U.S.A.). - Crotch, 1876:466. - Gemminger \& Harold, 1876:3698. - Kuhnt, 1909:86; 1911:61. - Boyle, 1956:101, 107, fig. 76, 93. - Johnson, 1967:5, 10. Distr.; Canadá. U.S.A. México, América Central.

hogei Gorham, 1888:78. - Johnson, 1967:10 (syn.).

championi Gorham, 1888:78, pl. 4, fig. 18. - Johnson, 1967:10 (syn.).

mesomelas Gorham, 1888:79. - Johnson, 1967:10 (syn.).

var. confinis LeConte, 1854:162. - Johnson, 1967:10 (syn.).

latipalpus Johnson, 1967:6, 11, fig. 4. Tp.: NMNH - (Brasil, Santa Catarina, Nova Teutônia). Distr.: Brasil, região sul.

mesosternalis Schaeffer, 1905:145. Tp.: NMNH - (U.S.A., Arizona, Cochise, Palmelee). - Kuhnt, 1909:86; 1911:62. - Boyle, 1956:101, 106, fig. 78, 94. - Johnson, 1967:5, 12. Distr.: U.S.A. e México.

monostigma Casey, 1916:165. - Boyle, 1956:106 (syn.).

coloradana Casey, 1924:179. - Boyle, 1956:106 (syn.).

rediviva Gorham, 1888:79, pl. 4, fig. 19. Tp.: NHML - (Guatemala, Quiché mts, 8000 fts.). - Kuhnt, 1909:86; 1911:63. - Blackwelder, 1945:468. - Johnson, 1967:6, 12, fig. 5-6. Distr.: Guatemala.

sola Johnson, 1967:6, 14, fig. 7. Tp. ? - (México, Querétaso, San Juan del Rio). Distr.: México.

valdiviana Philippi, 1864:401. Tp. ? -(Chile). - Gemminger \& Harold, 1876:3699. - Kuhnt, 1911:65. - Blackwelder, 1945:468. - Guérin, 1952:182, fig. 3. 
Distr.: Chile.

wehrlei Boyle, 1954a:260. Tp.: CAS - (U.S.A. Arizona, Santa Cruz, Patagonia); 1956:101, 108, fig. 84, 96. - Johnson, 1967:6, 15. Distr.: U.S.A. e México.

\section{Mycophtorus Lacordaire, 1842}

Mycophtorus Lacordaire, 1842:31, 193 (Tgen.: Mycophtorus melanocerus Lacordaire, 1842:193, seg. Crotch, 1876:470). - Chapuis, 1876:31, 34. - Crotch, 1876:470. - Gorham, 1888:73. - Kuhnt, 1909:57, 88. - Deelder, 1942:54. Alvarenga, 1965:86.

melanocerus Lacordaire, 1842:193. Tp. ? - (Caiena). - Crotch, 1876:471.

Gemminger \& Harold, 1876:3695. - Kuhnt, 1909:89; 1911:66. - Blackwelder, 1945:468. Distr.: Guiana Francesa.

pauperculus Lacordaire, 1842:194. Tp. ? - (Colômbia). - Crotch, 1876:471. -

Gemminger \& Harold, 1876:3695. - Gorham, 1888:74, pl. 4, fig. 13. Kuhnt, 1909:89; 1911:66. - Blackwelder, 1945:468. Distr.: América Central. Colômbia. Brasil, região amazônica.

peruvianus Kirsch, 1876:101. Tp.: SMTD exam. aut. - (Peru, Pozuzo). - Gemminger \& Harold, 1876:3695. - Kuhnt, 1909:89; 1911:66. - Mader, 1942d:176; 1951:224. - Blackwelder, 1945:468. Distr.: Peru.

vernix Casey, 1916:159. Tp.: NMNH - (Panamá). - Blackwelder, 1945:468. Distr.: Panamá.

\section{Pseudolybas Gorham, 1888}

Gorham, 1888:74 (Tgen.: Pseudolybas glaber Gorham, 1888:74, seg. Alvarenga, 1965:89). - Kuhnt, 1909:56, 89. - Deelder, 1942:53. - Alvarenga, 1965:89.

glaber Gorham, 1888:74, pl. 4, fïg. 14. Tp.: NHML - (Panamá, Bugaba). - Kuhnt, 1909:89; 1911:66. - Blackwelder, 1945:468. Distr.: Panamá.

vernicatus Gorham, 1888:75. Tp.: NHML - (Panamá, Bugaba, volcán de Chiriquí). - Kuhnt, 1909:89; 1911:66. - Blackwelder, 1945:468. Distr.: Panamá.

\section{Apolybas Alvarenga, 1965}

Apolybas Alvarenga, 1965:81 (Tgen.: Lybas normalis Lacordaire, 1842:235, desig. orig.).

anisotomoides Gorham, 1888:76 (Lybas). Tp.: NHML - (Panamá, volcán de Chi- 
riquí). - Kuhnt, 1909:90; 1911:66. - Blackwelder, 1945:468. Distr.: Panamá.

atripennis Erichson, 1847:180 (Lybas). Tp.: ZMHU exam. aut. - (Peru). Gemminger \& Harold, 1876:3701. - Kuhnt, 1909:90; 1910:248; 1911:66.

- Mader, 1942d:176; 1951:224. - Blackwelder, 1945:468. Distr.: Peru.

axillaris Lacordaire, 1842:236 (Lybas). Tp. ? - (Guiana Francesa). - Crotch, 1876:473. Gemminger \& Harold, 1876:3701. - Kuhnt, 1909:91; 1911:66.

- Curran, 1941:285. - Blackwelder, 1945:468. Distr.: Guiana Francesa.

bicolor Guérin-Méneville, 184 lb:153 (Brachymerus). Tp.: MCZC exam. aut. (Brasil). - Lacordaire, 1842:230 (Lybas). - Crotch, 1876:471. - Gemminger \& Harold, 1876:3701. - Kuhnt, 1909:90, pl. 3, fig. 11; 1911:66. Schenkling, 1919:86. - Mader, 1942d:176; 1951:224. - Blackwelder, 1945:468. - Guérin, 1948:21. Distr.: Venezuela. Colômbia. Equador. Peru. Bolívia. Brasil, região amazônica.

calidus Lacordaire, 1842:233 (Lybas). Tp. ? - (Colômbia). - Crotch, 1876:472. Gemminger \& Harold, 1876:3701. - Kuhnt, 1909:91; 1911:67. - Curran, 1941:285. - Blackwelder, 1945:468. Distr.: Colômbia. Brasil, região amazônica.

carbunculus Lacordaire, 1842:238 (Lybas). Tp.: MCZC exam. aut. - (México, Yucatán). - Crotch, 1876:473. - Gemminger \& Harold, 1876:3701. Gorham, 1888:76, pl. 4, fig. 16. - Kuhnt, 1909:90; 1911:67. - Blackwelder, 1945:468. Distr.: México, Guatemala. Panamá.

chlamydophorus Lacordaire, 1842:234 (Lybas). Tp. ? - (Colômbia). - Crotch, 1876:472. - Gemminger \& Harold, 1876:3701. - Kuhnt, 1909:91; 1911:67.

- Curran, 1941:285. - Blackwelder, 1945:468. Distr.: Colômbia.

corallinus Lacordaire, 1842:239 (Lybas). Tp.: MCZC exam. aut. - (Caiena). Crotch, 1876:473. - Gemminger \& Harold, 1876:3701. - Kuhnt, 1909:90; 1911:67. - Blackwelder, 1945:468. Distr.: Guiana Francesa.

cruentissimus Casey, 1916:170 (Lybas). Tp.: NMNH - (Guatemala, Quiriguá). Blackwelder, 1945:468. Distr.: Guatemala.

dorsalis Gorham, 1883:86 (Lybas). Tp.: NHML exam. aut. - (Peru, Chanchamayo). - Fleutiaux, 1886:223. - Kuhnt, 1909:91; 1911:67. Curran, 1941:285. - Mader, 1942d:176; 1951:224. - Blackwelder, 1945:468. Distr.: Peru.

faba Lacordaire, 1842:234 (Lybas). Tp.: MCZC exam. aut. - (Colômbia). Crotch, 1876:472. - Gemminger \& Harold, 1876:3701. - Kuhnt, 1909:90; 1911:67. - Blackwelder, 1945:468. Distr.: Colômbia.

ferrugineus Olivier, 1807:482, pl. 3, fig. 34 (Erotylus). Tp. ? - (Suriname). Lacordaire, 1842:230 (Lybas). - Crotch, 1876:471. - Gemminger \& Harold, 1876:3701. - Kuhnt, 1909:90; 1911:67. - Blackwelder, 1945:468. Distr.: Suriname. Guiana Francesa.

granatus Lacordaire, 1842:231 (Lybas). Tp. ? - (México, Yucatán). - Crotch, 
1876:472. - Gemminger \& Harold, 1876:3701. - Gorham, 1888:75, pl. 4, fig. 15; 1898:254. - Kuhnt, 1909:90; 1911:67. - Blackwelder, 1945:468. Distr.: México.

guianacus Curran, 1941:285, pl. 1, fig. 7 (Lybas). Tp.: DTNY - (Guiana, Kartabo). Distr.: Guiana.

humeralis Kuhnt, 1910:248 (Lybas). Tp.: ZMHU exam. aut. - (Guiana Francesa, St. Laurent); 1911:67. - Blackwelder, 1945:468. Distr.: Guiana Francesa. interpunctatus Gorham, 1888:76 (Lybas). Tp.: NHML - (México, Cerro de Plumas). - Kuhnt, 1909:90; 1911:67. - Blackwelder, 1945:468. Distr.: México.

kleinei Mader, 1943:115 (Lybas). Tp.: MGFM exam. aut. - (Peru, Pebas); 1951:224. Distr.: Peru.

mycetophilus Lacordaire, 1842:237 (Lybas). Tp. ? - (Caiena). - Crotch, 1876:473. - Gemminger \& Harold, 1876:3701. - Kuhnt, 1909:91; 1911:67. - Curran, 1941:285. Blackwelder, 1945:468. Distr.: Guiana Francesa.

normalis Lacordaire, 1842:235 (Lybas). Tp. ? - (Caiena). - Crotch, 1876:472. Gemminger \& Harold, 1876:3701. - Kuhnt, 1909:91; 1911:67. - Curran, 1941:285. - Blackwelder, 1945:468. Distr.: Guiana Francesa. Brasil, região amazônica.

pulicarius Lacordaire, 1842:238 (Lybas). Tp. ? - (Caiena). - Crotch, 1876:473. Gemminger \& Harold, 1876:3701. Kuhnt, 1909:91; 1911:67. - Curran, 1941:285. - Blackwelder, 1945:468. Distr.: Guiana Francesa.

puncticollis Mader, 1942d:176, 200 (Lybas). Tp.: DEIB - (Peru, Cushi); 1951: 224. Distr.: Peru.

rufinus Lacordaire, 1842:232 (Lybas). Tp. ? - (Brasil). - Crotch, 1876:472. Gemminger \& Harold, 1876:3701. - Kuhnt, 1909:90; 1911:67. - Blackwelder, 1945:468. Distr.: Guiana Francesa. Brasil.

seminiger Delkeskamp, 1957:99, 114 (Lybas). Tp.: ZSBS - (Bolívia, Sarampiuni, San Carlos, $1000 \mathrm{~m})$. Distr.: Bolívia.

seminulus Lacordaire, 1842:237 (Lybas). Tp. ? - (Caiena). - Crotch, 1876:473. Gemminger \& Harold, 1876:3701. - Kuhnt, 1909:91; 1911:67. - Curran, 1941:285. - Blackwelder, 1945:468. Distr.: Guiana Francesa.

thoracicus Olivier, 1807:486, pl. 3, fig. 41 (Erotylus). Tp.: MCZC exam. aut. (Guiana Francesa). - Duponchel, 1825:174. - Lacordaire, 1842:236 (Lybas). - Crotch, 1876:472. - Gemminger \& Harold, 1876:3701. - Kuhnt, 1909:90; 1911:67. - Balckwelder, 1945:468. Distr.: Guiana Francesa.

triangularis Curran, 1941:285, pl. 1, fig. 6 (Lybas). Tp.: DTNY - (Guiana, Kartabo). Distr.: Guiana. 


\section{Lybanoides Gorham, 1888}

Lybanoides Gorham, 1888:77 (Tgen.: Lybanoides castaneus Gorham, 1888:77, monotipia). - Kuhnt, 1909:56, 91. - Deelder, 1942:53. - Alvarenga, 1965:85.

castaneus Gorham, 1888:77, pl. 4, fig. 17. Tp.: NHML exam. aut. - (Nicarágua, Chontales). - Kuhnt, 1909:91; 1911:67. - Blackwelder, 1945:468. Distr.: Nicarágua.

\section{Mycolybas Crotch, 1876}

Mycolybas Crotch, 1876:473 (Tgen.: Lybas lucidus Lacordaire, 1842:232, desig. orig.). - Kuhnt, 1909:56, 91. - Deelder, 1942:53. - Alvarenga, 1965:86.

coccineipennis Motschulsky, 1858:116 (Ischyrus). Tp. ? - (América Central). Crotch, 1876:474 (Mycolybas ?). - Gemminger \& Harold, 1876:3690. Kuhnt, 1909:92; 1911:67. - Blackwelder, 1945:468. Distr.: América Central.

cruentatus Kirsch, 1865:97 (Lybas). Tp.: SMTD exam. aut. - (Colômbia, Bogotá). - Crotch, 1876:474 (Mycolybas). - Gemminger \& Harold, 1876:3701. Kuhnt, 1909:92; 1911:67. - Blackwelder, 1945:468. Distr.: Colômbia. Brasil.

egae Crotch, 1876:474. Tp.: MCZC exam. aut. - (Ega)[atualmente Tefé].

Gemminger \& Harold, 1876:3701. Kuhnt, 1909:92; 1911:67. - Blackwelder, 1945:468. Distr.: Brasil, região amazônica.

lucidus Lacordaire, 1842:232 (Lybas). Tp.: MCZC exam. aut. - (Brasil). - Crotch, 1876:474 (Mycolybas). - Gemminger \& Harold, 1876:3701. - Kuhnt, 1909:92; 1911:67. - Bruch, 1914:384. - Deelder, 1942:96. - Blackwelder, 1945:468. - Guérin, 1948:21. Distr.: Brasil, regiões sudeste e sul. Argentina.

sachi Mader, 1938:19 (Mycomystes). - Delkeskamp, 1939:29. (syn.).

melanocorynus Lacordaire, 1842:233 (Lybas). Tp. ? - (Brasil). - Crotch, 1876:474 (Mycolybas). - Gemminger \& Harold, 1876:3701. - Kuhnt, 1909:92; 1911:67. - Blackwelder, 1945:468. Distr.: Brasil.

rufipennis Guérin, 1956:63, fig. 27. Tp.: CDSP exam. aut. - (Brasil, Amazonas, Manaus). Distr.: Brasil, região amazônica.

sanguinosus Motschulsky, 1858:116 (Ischyrus). Tp. ? - (Colômbia). - Crotch, 1876:474 (Mycolybas ?). - Gemminger \& Harold, 1876:3691. - Kuhnt, 1909:91; 1911:67. - Blackwelder, 1945:468. Distr.: Colômbia. 


\section{Neoxestus Crotch, 1876}

Neoxestus Crotch, 1876:476 (Tgen.: Neoxestus chilensis Crotch, 1876:476, monotipia). - Kuhnt, 1909:57, 93. - Deelder, 1942:54. - Guérin, 1952:180. Alvarenga, 1965:87.

chilensis Crotch, 1876:476. Tp.: MCZC exam. aut. - (Chile). - Gemminger \& Harold, 1876:3696. - Kuhnt, 1909:93; 1911:68. - Blackwelder, 1945:468. - Guérin, 1952:183, fig. 6. Distr.: Chile.

\section{ERotylinae Crotch, 1876}

Lacordaire, 1842:243-518 (Erotyliens vrais). - Chapuis, 1876:48-72 (Erotylites). - Crotch, 1876:481-562 (Erotylini). - Gorham, 1888:82-114 (Erotylides). Kuhtn, 1909:7-51 (Erotylini). - Boyle, 1956:78, 93-96 (Erotylinae).

\section{Thonius Lacordaire, 1842}

Thonius Lacordaire, 1842:31, 252 (Tgen.: Thonius pavoninus Lacordaire, 1842: 253, seg. Crotch, 1876:481). - Chapuis, 1876:49, 51. - Crotch, 1876:481. Kuhnt, 1909:9, 12. - Deelder, 1942:51. - Alvarenga, 1965:90.

buckleyi Crotch, 1876:482. Tp.: MCZC exam. aut. - (Equador). - Gemminger \& Harold, 1876:3703. - Kuhnt, 1909:13; 1911:5. - Blackwelder, 1945:456. Distr.: Equador.

flavipennis Guérin-Méneville, 1844:309. Tp. ? - (Colômbia). - Crotch, 1876:482. - Gemminger \& Harold, 1876:3703. - Kuhnt, 1909:13; 1911:5. - Blackwelder, 1945:456. Distr.: Colômbia.

flexuososignatus Crotch, 1876:482. Tp.: MCZC exam. aut. - (Colômbia). Gemminger \& Harold, 1876:3703. - Kuhnt, 1909:13; 1911:6. - Blackwelder, 1945:456. Distr.: Colômbia.

maculatus Guérin-Méneville, 1844:308. Tp.: MNHN exam. aut. - (Colômbia). Lacordaire, 1876, pl. 132, fig. 3. - Crotch, 1876:481. - Gemminger \& Harold, 1876:3703. - Kuhnt, 1909:13; 1911:6. - Blackwelder, 1945:456. Distr.: Colômbia.

nebrioides Crotch, 1876:482. Tp.: MCZC exam. aut. - (Colômbia). - Gemminger \& Harold, 1876:3703. - Kuhnt, 1909:13; 1911:6. - Blackwelder, 1945:456. Distr.: Colômbia.

pavoninus Lacordaire, 1842:253. Tp.: MCZC exam. aut. - (Colômbia). - Crotch, 1876:481. - Gemminger \& Harold, 1876:3703. - Kuhnt, 1909:13; 1911:6. - Deelder, 1942:56. - Blackwelder, 1945:456. Distr.: Colômbia.

substriatus Crotch, 1876:482. Tp.: MCZC exam. aut. - (Colômbia). - Gemminger \& Harold, 1876:3703. - Kuhnt, 1909:13; 1911:6. - Blackwelder, 1945:456. 
Distr.: Colômbia.

unicolor Guérin-Méneville, 1844:309. Tp.: MNHN exam. aut. - (Colômbia); 1855:610. - Crotch, 1876:482. - Gemminger \& Harold, 1876:3703. Kuhnt, 1909:13; 1911:6. - Mader, 1943:109. - Blackwelder, 1945:456. Distr.: Colômbia. Equador.

\section{Euphanistes Lacordaire, 1842}

Euphanistes Lacordaire, 1842:31, 255 (Tgen.: Euphanistes hydrophiloides Lacordaire, 1842:256, seg. Crotch, 1876:483). - Chapuis, 1876:49, 52. - Crotch, 1876:483. - Kuhnt, 1909:9, 13. - Deelder, 1942:51. - Alvarenga, 1965:84.

hydrophiloides Lacordaire, 1842:256. Tp. ? - (Colômbia). - Crotch, 1876:483. Gemminger \& Harold, 1876:3703. - Kuhnt, 1909:14; 1911:6. - Blackwelder, 1945:456. Distr.: Colômbia.

\section{Cyclomorphus Hope, 1841}

Cyclomorphus Hope, 1841:114 (Tgen.: Cyclomorphus globosus Guérin-Méneville, 1841:120, desig. orig.). - Lacordaire, 1842:31, 258. - Chapuis, 1876: 49, 53. - Crotch, 1876:483. - Gorham, 1898:254. - Kuhnt, 1909:8, 14. Deelder, 1942:51. - Alvarenga, 1965:83.

aeneomaculatus Crotch, 1876:486. Tp.: MCZC exam. aut. - (Colômbia). Gemminger \& Harold, 1876:3703. - Kuhnt, 1909:15; 1911:6. - Blackwelder, 1945:456. Distr.: Colômbia.

alienus Crotch, 1876:484. Tp.: MCZC exam. aut. - (Colômbia). - Gemminger \& Harold, 1876:3703. - Kuhnt, 1909:15; 1911:6. - Blackwelder, 1945:456. Distr.: Colômbia.

apicicornis Crotch, 1876:486. Tp.: MCZC exam. aut. - (Colômbia). - Gemminger \& Harold, 1876:3703. - Kuhnt, 1909:15; 1911:6. - Blackwelder, 1945:456. Distr.: Colômbia.

beauvoisi Lacordaire, 1842:259. Tp. ? - (Colômbia). - Crotch, 1876:483. Gemminger \& Harold, 1876:3703. - Kuhnt, 1909:15; 1911:6. - Blackwelder, 1945:456. Distr.: Colômbia.

bihamatus Lacordaire, 1842:414 (Brachysphoenus). Tp. ? (Colômbia). - Crotch, 1876:487 (Cyclomorphus). - Gemminger \& Harold, 1876:3703. - Kuhnt, 1909:15; 1911:6. - Blackwelder, 1945:456. Distr.: Colômbia.

bisbimaculatus Lacordaire, 1842:265. Tp. ? - (Colômbia). - Crotch, 1876:485. Gemminger \& Harold, 1876:3703. - Kuhnt, 1909:14; 1911:6. - Deelder, 1942:56. - Blackwelder, 1945:456. Distr.: Colômbia.

bonplandi Lacordaire, 1842:263. Tp. ? - (Colômbia). - Crotch, 1876:484. 
Gemminger \& Harold, 1876:3703. - Kuhnt, 1909:14; 1911:6. - Blackwelder, 1945:456. Distr.: Colômbia.

chrysomelinus Lacordaire, 1842:191 (Mycotretus). Tp. ? - (Colômbia). - Crotch, 1876:487 (Cyclomorphus). - Gemminger \& Harold, 1876:3703. - Kuhnt, 1909:15; 1911:6. - Blackwelder, 1945:456. Distr.: Colômbia.

coccinellinus Crotch, 1876:486. Tp.: MCZC exam. aut. - (Colômbia). - Gemminger \& Harold, 1876:3703. - Kuhnt, 1909:15; 1911:6. - Blackwelder, 1945:456. Distr.: Colômbia.

extricatus Crotch, 1876:485. Tp.: MCZC exam. aut. - (Bogotá). - Gemminger \& Harold, 1876:3703. - Kuhnt, 1909:14; 1911:6. - Blackwelder, 1945:456. Distr.: Colômbia.

gibbosus Crotch, 1876:486. Tp.: MCZC exam. aut. - (Colômbia). - Gemminger \& Harold, 1876:3703. - Kuhnt, 1909:15; 1911:6. - Blackwelder, 1945:456. Distr.: Colômbia.

glabratus Waterhouse, 1879:428, pl. 9, fig. 5. Tp.: NHML - (Colômbia, Medellín). - Fleutiaux, 1886:224. - Kuhnt, 1909:14; 1911:6. - Blackwelder, 1945:456. Distr.: Colômbia.

globosus Guérin-Méneville, 1841a:120. Tp.: MCZC exam. aut. - (Colômbia). Lacordaire, 1842:261. - Crotch, 1876:484. - Gemminger \& Harold, 1876:3704. - Kuhnt, 1909:14; 1911:6. - Deelder, 1942:56. - Blackwelder, 1945:456. Distr.: Colômbia.

humboldti Lacordaire, 1842:263. Tp. ? - (Colômbia). - Crotch, 1876:484. Gemminger \& Harold, 1876:3704. - Kuhnt, 1909:14; 1911:6. - Blackwelder, 1945:456. Distr.: Colômbia.

infaustus Lacordaire, 1842:265. - Crotch, 1876:484 (syn.).

inflatus Lacordaire, 1842:262. Tp. ? - (Colômbia). - Crotch, 1876:484. - Gemminger \& Harold, 1876:3704. - Kuhnt, 1909:14; 1911:6. Blackwelder, 1945:456. Distr.: Colômbia.

iphicloides Crotch, 1876:485. Tp.: MCZC exam. aut. - (Equador). - Gemminger \& Harold, 1876:3704. - Kuhnt, 1909:14; 1911:6. - Blackwelder, 1945:456.

Distr.: Equador.

laeviusculus Crotch, 1876:483. Tp.: MCZC exam. aut. - (Venezuela). - Gemminger \& Harold, 1876:3704. - Kuhnt, 1909:15; 1911:6. - Blackwelder, 1945:456. Distr.: Venezuela.

maeander Lacordaire, 1842:260. Tp. ? - (Colômbia). - Crotch, 1876:483. Gemminger \& Harold, 1876:3704. - Kuhnt, 1909:15; 1911:6. Deelder, 1942:56. Distr.: Colômbia.

octopunctatus Erichson, 1847:179. Tp.: exam. aut. - (Peru). - Crotch, 1876:487. - Gemminger \& Harold, 1876:3704. - Kuhnt, 1909:15; 1911:6. - Mader, 1942d:150; 1944:95. - Blackwelder, 1945:456. Distr. Peru. Bolívia.

pertinax Lacordaire, 1842:336 (Brachysphoenus). Tp.: MCZC exam. aut. - 
(Colômbia). - Crotch, 1876:485 (Cyclomorphus). - Gemminger \& Harold, 1876:3704. - Kuhnt, 1909:14; 1911:6. - Blackwelder, 1945:456. Distr.: Colômbia.

quadriplagiatus Lacordaire, 1842:264. Tp. ? - (Colômbia). - Crotch, 1876:485.

- Gemminger \& Harold, 1876:3704. - Kuhnt, 1909:15; 1911:6. - Blackwelder, 1945:456. Distr.: Colômbia.

sicardi Kuhnt, 1910:248. Tp.: ZMHU exam. aut. - (Peru, Cushi); 1911:6. Mader, 1942d:150; 1944:95. - Blackwelder, 1945:456. Distr.: Peru.

sordidus Gorham, 1898:254. Tp.: NHML exam. aut. - (México, Guerrero, Omilteme). - Kuhnt, 1909:14; 1911:7. - Blackwelder, 1945:456. Distr.: México.

subocellatus Crotch, 1876:485. Tp.: MCZC exam. aut. - (Colômbia). - Gemminger \& Harold, 1876:3704. - Kuhnt, 1909:14; 1911:7. - Blackwelder, 1945:456. Distr.: Colômbia.

tumidus Lacordaire, 1842:261. Tp. ? - (Colômbia). - Crotch, 1876:483. Gemminger \& Harold, 1876:3704. - Kuhnt, 1909:15; 1911:7. - Blackwelder, 1945:456. Distr.: Colômbia.

variegatus Kirsch, 1865:98. Tp.: SMTD exam. aut. - (Bogotá). - Crotch, 1876: 487. - Gemminger \& Harold, 1876:3704. - Kuhnt, 1909:14; 1911:7. Blackwelder, 1945:456. Distr.: Colômbia.

\section{Scaphengis Gorham, 1888}

Scaphengis Gorham, 1888:82 (Tgen.: Scaphengis picipes Gorham, 1888:83, monotipia). - Kuhnt, 1909:8, 15. - Deelder, 1942:51. - Alvarenga, 1965:89.

picipes Gorham, 1888:83, pl. 5, fig. 6. Tp.: NHML - (México, Toxpam). - Kuhnt, 1909:15; 1911:7. - Blackwelder, 1945:456. Distr.: México.

\section{Strongylosomus Chevrolat, 1837}

Strongylosomus Chevrolat, 1837:427 (Tgen.: Erotylus unicolor Olivier, 1807:481, seg. Crotch, 1876:487). - Crotch, 1876:487. - Alvarenga, 1965:90.

Coccimorphus Hope, 1841:114 (Tgen.: Erotylus unicolor Olivier, 1807:481, desig. orig.). - Lacordaire, 1842:32, 266. - Chapuis, 1876:49, 54. - Gorham, 1888:83. - Kuhnt, 1909:9, 15. - Deelder, 1942:51. - Alvarenga, 1965:90 (syn.).

alutaceus Erichson, 1847:179 (Coccimorphus). Tp. ? - (Peru). - Gemminger \& Harold, 1876:3704. - Kuhnt, 1909:16; 1911:7. - Mader, 1942d:150; 1944:95. - Blackwelder, 1945:456. - Crotch, 1876:489 (Strongylosomus). Distr.: Peru. 
bolivianus Schenkling, 1919:80 (Aegithus). Tp.: DEME exam. aut. - (Bolívia). Blackwelder, 1945:456. - Mader, 1942c:550 (Coccimorphus). Distr.: Bolívia.

capitatus Lacordaire, 1842:270 (Coccimorphus. Tp. ? - (Brasil). - Gemminger \& Harold, 1876:3704. - Kuhnt, 1909:16; 1911:7. - Blackwelder, 1945:456. - Crotch, 1876:488 (Strongylosomus). Distr.: Brasil, região amazônica.

carmineus Lacordaire, 1842:269 (Coccimorphus). Tp. ? - (Brasil). - Gemminger \& Harold, 1876:3704. - Kuhnt, 1909:16; 1911:7. - Blackwelder, 1945:456. - Crotch, 1876:487 (Strongylosomus). Distr.: Brasil.

coccinelloides Duponchel, 1825:157, pl. 7, fig. 63 (Erotylus). Tp. ? (Brasil). Lacordaire, 1842:272 (Coccimorphus). - Guérin-Méneville, 1855:610. Gemminger \& Harold, 1876:3704. - Kuhnt, 1909:16; 1911:7. - Blackwelder, 1945:456. - Crotch, 1876:488 (Strongylosomus). Distr.: Brasil.

cribripennis Achard, 1926:143 (Coccimorphus). Tp. ? - (Argentina, Missiones). - Blackwelder, 1945:456. Distr.: Argentina.

dichrous Lacordaire, 1842:274 (Coccimorphus). Tp. ? - (Colômbia, rio Madalena). - Gemminger \& Harold, 1876:3704. - Gorham, 1888:84. - Kuhnt, 1909:16, 1911:7. - Declder, 1942:57. - Mader, 1942d:150; 1944:95. Blackwelder, 1945:456. - Crotch, 1876:489 (Strongylosomus). Distr.: Da América Central até o Brasil, região amazônica.

emys Lacordaire, 1842:518 (Coccimorphus). Tp. ? - (México, Oaxaca). - Gemminger \& Harold, 1876:3704. - Gorham, 1888:84, pl. 4, fig. 21. - Kuhnt, 1909:16; 1911:7. - Deelder, 1942:57. - Blackwelder, 1945:456. - Crotch, 1876:488 (Strongylosomus). Distr.: México.

floripesae Alvarenga, 1962:11, fig. 1 (Coccimorphus). Tp. perdido - (Brasil, Mato Grosso, P.N. Xingu). Distr.: Brasil, região central.

foveicollis Lacordaire, 1842:275 (Coccimorphus). Tp. ? - (Brasil). - Gemminger \& Harold, 1876:3704. - Kuhnt, 1909:16; 1911:7. - Blackwelder, 1945:456.

- Crotch, 1876:489 (Strongylosomus). Distr.: Brasil.

frenatus Guérin-Méneville, $1841 \mathrm{a} 120$ (Coccimorphus). Tp. ? - (Colômbia). Lacordaire, 1842:271. - Gemminger \& Harold, 1876:3704. - Gorham, 1888:84. - Kuhnt, 1909:16; 1911:7. - Blackwelder, 1945:456. - Crotch, 1876:488 (Strongylosomus). Distr.: Nicarágua. Colômbia.

melanopus Lacordaire, 1842:269 (Coccimorphus). Tp.: MCZC exam. aut. (Caiena). - Gemminger \& Harold, 1876:3704. - Kuhnt, 1909:16; 1911:7.

- Deelder, 1942:57. - Blackwelder, 1945:456. Guérin, 1948:5. - Crotch, 1876:487 (Strogylosomus). Distr.: Guiana Francesa. Brasil, região sudeste. nigripes Duponchel, 1825:158, pl. 7, fig. 65 (Erotylus). Tp.: MNHN exam. aut. - (Brasil). - Lacordaire, 1842:268 (Coccimorphus). - Gemminger \& Harold, 1876:3704. - Kuhnt, 1909:16; 1911:7. - Blackwelder, 1945:456. Crotch, 1876:487 (Strongylosomus). Distr.: Brasil.

nigrotibialis Mader, 1942c:549 (Coccimorphus). Tp.: DEIB - (Paraguai). - Distr.: 
Paraguai.

peruvianus Crotch, 1876:488 (Strongylosomus). Tp.: MCZC exam. aut. - (Peru). - Gemminger \& Harold, 1876:3704 (Coccimorphus). - Kuhnt, 1909:16; 1911:7. - Mader, 1942c:150; 1944:95. - Blackwelder, 1945:456. Distr.: Peru.

pictus Schenkling, 1919:80 (Aegithus). Tp.: DEME exam. aut. - (Panamá, Chiquirí). - Blackwelder, 1945:457. - Mader, 1942c:550 (Coccimorphus). Distr.: Panamá.

rotundatus Lacordaire, 1842:270 (Coccimorphus). Tp. ? - (Caiena). - Gemminger \& Harold, 1876:3704. - Kuhnt, 1909:16; 1911:7. - Blackwelder, 1945:456. Crotch, 1876:488 (Strongylosomus). Distr.: Guiana Francesa.

rugosus Lacordaire, 1842:276 (Coccimorphus). Tp.: MCZC exam. aut. - (Caiena). - Gemminger \& Harold, 1876:3704. - Kuhnt, 1909:16, pl. 3, fig. 1; 1911:7. - Bruch, 1914:379. - Schenkling, 1919:79. - Deelder, 1942:57. Blackwelder, 1945:456. - Guérin, 1948:5. - Delkeskamp, 1957:96. Crotch, $1876: 489$ (Strongylosomus). Distr.: Do Panamá até a Argentina.

simplex Guérin, 1956:45, fig. 1 (Coccimorphus). Tp.: CDSP exam. aut. - (Brasil, Amazonas, São Paulo de Olivença). Distr.: Brasil, região amazônica.

unicolor Olivier, 1807:481, pl. 3, fig. 32 (Erotylus). Tp. ? - (Guiana Francesa). - Lacordaire, 1842:272 (Coccimorphus). - Guérin-Méneville, 1855:610. Gemminger \& Harold, 1876:3704. - Kuhnt, 1909:16; 1911:7. - Bruch, 1914:379. - Deelder, 1942:57. - Blackwelder, 1945:456. - Guérin, 1948:5. - Crotch, 1876:489 (Strongylosomus). Distr.: Guiana Francesa. Brasil. Argentina.

brevicornis Duponchel, 1825:160, pl. 7, fig. 68. - Lacordaire, 1842:272 (syn.).

\section{Plastococcus Gorham, 1898}

Plastococcus Gorham, 1898:255 (Tgen.: Plastococcus atricinctus Gorham, 1898: 255, seg. Alvarenga, 1965:88). - Kuhnt, 1909:9, 17. - Deelder, 1942:51. Alvarenga, 1965:88.

apicalis Gorham, 1898:256. Tp.: NHML exam. aut. - (Panamá, Chiriquí, San Felix). - Kuhnt, 1909:17; 1911:8. - Blackwelder, 1945:456. Distr.: Panamá.

atricinctus Gorham, 1898:255. Tp.: NHML exam. aut. - (Panamá, Bugaba, David, volcán de Chiriquí). - Kuhnt, 1909:17; 1911:8. - Blackwelder, 1945:456. Distr.: Panamá. 


\section{Aegithus Fabricius, 1801}

Aegithus Fabricius, 1801:9 (Tgen.: Chrysomela clavicornis Linnaeus, 1758:370, seg. Hope, 1841:114). - Hope, 1841:114. - Lacordaire, 1842:32, 276. Crotch, 1873b:145; 1876:489. - Chapuis, 1876:49, 55. - Gorham, 1888:85.

- Kuhnt, 1909:9, 17. - Deelder, 1942:51. - Alvarenga, 1965:81. Aegithomimus Mader, 1942d:177. - Alvarenga, 1977:105 (syn.).

abruptus Casey, 1916:170. Tp.: NMNH - (México, Guerrero, rio Balsas). Blackwelder, 1945:456. Distr.: México.

andreae Lacordaire, 1842:282. Tp. ? - (Colômbia). - Crotch, 1876:492. Gemminger \& Harold, 1876:3704. - Kuhnt, 1909:19, pl. 2, figs 3, 3a, 3b; 1911:8 - Deelder, 1942:57. - Blackwelder, 1945:456. Distr.: Colômbia. Suriname. Brasil.

var. denticulatus Deelder, 1945:57, fig. 1a. Tp.: RNHL - (Suriname). Distr.: Suriname.

var. quadrimaculatus Guérin, 1956:46. Tp.: CDSP exam. aut. - (Brasil, Estado São Paulo, Batatais). Distr.: Brasil, região sudeste.

sexmaculatus Kirsch, 1865:99. - Crotch, 1876:492 (syn.).

armitagei Gorham, 1889:619. - Tp.: NHML exam. aut. - (Brasil, Amazonas). Kuhnt, 1909:19; 1911:8. - Deelder, 1942:57. - Mader, 1942d:153; 1944: 98. - Blackwelder, 1945:456. Distr.: Colômbia. Peru. Brasil, região amazônica.

assimilis Crotch, 1876:495. Tp. ? - (Amazonas). - Gemminger \& Harold, 1876:3705. - Kuhnt, 1909:19; 1911:8. - Blackwelder, 1945:456. Distr.: Brasil, região amazônica.

bartletti Gorham, 1889:619, pl. 61, figs 9, 9a. Tp.: NHML exam. aut. - (Peru, região sudeste), - Kuhnt, 1909:19; 1911:8. - Mader, 1942d:153; 1944:98.

- Blackwelder, 1945:456. Distr.: Equador. Peru.

bicolor Kirsch, 1865:99. Tp.: SMTD exam. aut. - (Colômbia, Bogotá). - Crotch, 1876:496. - Gemminger \& Harold, 1876:3705. - Kuhnt, 1909:19; 1911:8. - Blackwelder, 1945:456. Distr.: Colômbia.

binarius Casey, 1916:169. Tp.: NMNH - (México, Guerrero). Blackwelder, 1945:456. Distr.: México.

bourcieri Guérin-Méneville, 1855:610. Tp.: MNHN exam. aut. - (Equador, rio Napo). - Crotch, 1876:496. - Gemminger \& Harold, 1876:3705. - Kuhnt, 1911:8. - Blackwelder, 1945:456. Distr.: Equador.

brunnipennis Lacordaire, 1842:285. Tp. ? - (Brasil). - Crotch, 1876:491. Gemminger \& Harold, 1876:3705. - Kuhnt, 1909:19; 1911:8. - Bruch, 1914:379. - Deelder, 1942:57. - Mader, 1942d:153; 1944:98. - Blackwelder, 1945:456. Distr.: Peru. Bolívia. Paraguai. Brasil. Argentina.

bulla Lacordaire, 1842:289. - Tp. ? - (Caiena). - Crotch, 1876:494. - Gemminger 
\& Harold, 1876:3705. - Kuhnt, 1909:19; 1911:8. - Blackwelder, 1945:456. Distr.: Guiana Francesa. Brasil, região amazônica.

burmeisteri Lacordaire, 1842:281. Tp. ? - (Bolívia, Santa Cruz de la Sierra); 1876, pl. 132, fig. 5. - Crotch, 1876:491. - Gemminger \& Harold, 1876:3705. Kuhnt, 1909:18; 1911:8. - Deelder, 1942:57. - Mader, 1942d: 153; 1944: 98. - Blackwelder, 1945:456. - Guérin, 1948:5. Distr.: América do Sul, região amazônica.

cardinalis Chevrolat, 1834, fasc. 4, Tp.: MCZC exam. aut. - (México, volcán Orizaba). - Lacordaire, 1842:284. - Crotch, 1873b:146; 1876:492. Gemminger \& Harold, 1876:3705. - Gorham, 1888:86, pl. 4, fig. 23. Kuhnt, 1909:18; 1911:8. - Deelder, 1942:57. - Blackwelder, 1945:457. Distr.: México. América Central.

var. minor Gorham, 1888:86. Tp.: BMBH - (México, Jalapa). - Kuhnt, 1909:18; 1911:8. - Blackwelder, 1945:457. Distr.: México.

chalybeus Duponchel, 1825:157, pl. 7, fig. 62 (Erotylus). Tp. ? - [Localidade não citada]. - Lacordaire, 1842:279 (Aegithus). - Crotch, 1876:489. - Gemminger \& Harold, 1876:3705. - Kuhnt, 1909:17; 1911:8. - Bruch, 1914: 179. - Deelder, 1942:57. - Mader, 1942d:151; 1944:96. - Blackwelder, 1945:457. Distr.: Brasil, regiões sudeste e sul. Paraguai. Argentina.

cinctipennis Duponchel, 1825:157, pl. 7, fig. 60 (Erotylus). Tp. ? - [Localidade não citada]. - Lacordaire, 1842:280 (Aegithus). - Crotch, 1876:490. Gemminger \& Harold, 1876:3705. - Kuhnt, 1909:18. - 1911:8. - Deelder, 1942:57. - Blackwelder, 1945:457. Distr.: Brasil, região sudeste.

circumfusus Kuhnt, 1908b:627. Tp.: ZMHU exam. aut. - (Peru, Chanchamayo); 1909:19; 1911:8. - Mader, 1942d:153; 1944:98. - Blackwelder, 1945:457. Distr.: Peru. Bolívia.

clathraticollis Mader, 1942c:551, 553. Tp.: MGFM exam. aut. - (Paraguai, Assunción). Distr.: Paraguai.

clavicornis Linnaeus, 1758:370 (Chrysomela). Tp. ? - (América). - Degeer, 1778:418, pl. 16, fig. 11. - Chevrolat, 1834 (Aegithus). - Crotch, 1873b: 147; 1876:493. - Gemminger \& Harold, 1876:3705. - Gorham, 1888:87. - Kuhnt, 1909:18; 1911:8. - Bruch, 1914:379. - Schenkling, 1919:79. Deelder, 1942:57. - Mader, 1942d:152; 1944:97. - Blackwelder, 1945:457. - Guérin, 1948:5. - Delkeskamp, 1957:96. Distr.: Do México até a Argentina.

surinamensis Linnaeus, 1763:10 (Coccinella). - Fabricius, 1775:79; 1781:93; 1787: 53; 1792:266. - Gmelin, 1890: 1645. - Fabricius, 1792: 39 (Erotylus). - Olivier, 1792:435; 1807:480, pl. 1, fig. 9. - Herbst, 1799:373, pl. 137, fig. 12. - Latreille, 1804:37. - Schönherr, 1808:328. - Fabricius, 1801:9 (Aegithus). - Illiger, 1804:160. - Sturm, 1826:139. - Lacordaire, 1842:285. - Guérin-Méneville, 1844, pl. 50, fig. 2; 1855:611. - Kirsch, 1876:102 (syn.).

consularis Guérin-Méneville, 1855:610. Tp.: MNHN ex aut. - (Brasil, Ama- 
zonas). - Crotch, 1876:490. - Kirsch, 1876:102. - Gemminger \& Harold, 1876:3705. - Kuhnt, 1909:18; 1911;9. - Mader, 1942d:151; 1944:96. Blackwelder, 1945;457. - Guérin, 1948:5. Distr.: Peru. Equador. Brasil, região amazônica.

cordatus Crotch, 1876:493. Tp.: MCZC exam. aut. - (Amazonas). - Gemminger \& Harold, 1876:3705. - Kuhnt, 1909:19; 1911:9. - Blackwelder, 1945:457. Distr.: Brasil, região amazônica.

cretaceus Guérin, 1956:46, fig. 2. Tp.: CDSP exam. aut. - (Brasil, Amazonas, Benjamin Constant). Distr.: Brasil, região amazônica.

cribrosus Lacordaire, 1842:293. Tp. ? - (Brasil). - Crotch, 1876:491. - Gemminger \& Harold, 1876:3705. - Kuhnt, 1909:18; 1911:9. - Mader, 1942d:152; 1944:97. - Blackwelder, 1945:457. Distr.: Equador. Peru. Bolívia. Brasil, região amazônica.

cyanipennis Guérin-Méneville, 1841a:120. Tp.: MCZC exam. aut. - (Bolívia); 1855:610. - Lacordaire, 1842:279. - Crotch, 1876:490. - Gemminger \& Harold, 1876:3705. - Dohrn, 1880:152. - Kuhnt, 1909:18; 1911:9. Brèthes, 1920:48. - Deelder, 1942:59. - Mader, 1942d:151; 1944:96. Blackwelder, 1945:457. - Guérin, 1948:5. - Delkeskamp, 1957:96. Distr.: Equador. Peru. Bolívia. Brasil, região amazônica.

var. viridis Brèthes, 1920:48. Tp. ? - (Peru, Chanchamayo). - Deelder, 1942:59. - Mader, 1942d:151. - Blackwelder, 1945:457. Distr.: Peru. decoloratus Kuhnt, 1910:249, fig. 13. Tp.: ZMHU exam. aut. - (Colômbia, El Tambo, Bogotá); 1911:9. - Blackwelder, 1945:457. Distr.: Colômbia.

dichrous Crotch, 1876:495. Tp.: MCZC exam. aut. - (Amazonas). - Gemminger \& Harold, 1876:3705. - Kuhnt, 1909:19; 1911:9. - Blackwelder, 1945:457. Distr.: Brasil, região amazônica.

dohrni Mader, 1942d:152, 176. Tp. ? - (Peru, Marcapata); 1944:97. Distr.: Peru. dubius Gorham, 1888:90. Tp.: NHML exam. aut. - (Panamá, Bugaba, Caldera). - Kuhnt, 1909:19; 1911:9. - Blackwelder, 1945:457. Distr.: Panamá.

duplicatus Gorham, 1888:89. Tp.: NHML exam. aut.- (Guatemala, Capetilo). Kuhnt, 1909:19; 1911:9. - Blackwelder, 1945:457. Distr.: Guatemala. Nicarágua.

ecuadoricus Mader, 1942c:550. Tp.: MGFM exam. aut. - (Equador, Loreto); 1944:96. Distr.: Equador.

flavolineatus Mader, 1943:109. Tp.: SMTD exam, aut. -(Bolivia). Distr.: Bolívia. geminatus Lacordaire, 1842:287. Tp. ? - (Rio de Janeiro). - Crotch, 1876:494. Gemminger \& Harold, 1876:3705. - Kuhnt, 1909:19; 1011:9. - Deelder, 1942:59. - Blackwelder, 1945:457. - Guérin, 1948:5. Distr.: Brasil, região sudeste.

globulus Achard, 1926:144. Tp. ? - (Equador). - Blackwelder, 1945:457. Distr.: Equador. 
gorhami Alvarenga, 1977:105. Distr.: Costa Rica.

discoideus; Gorham, 1888, nec Fabricius, 1801. - Gorham, 1888:88, pl. 5, fig. 2. Tp.: NHML exam. aut. - (Costa Rica, Cachí). - Kuhnt, 1909:19; 1911:9. - Blackwelder, 1945: 457. - Alvarenga, 1977:105 (nom. praeoc.).

grammicus Gorham, 1888:91, pl. 5, fig. 3. Tp.: NHML exam. aut. - (Guatemala, Vera Paz, San Juan). - Kuhnt, 1909:18; 1911:9. - Blackwelder, 1945:457.

Distr.: Guatemala.

hemisphaericus Lacordaire, 1842:289. Tp. ? - (Brasil). - Crotch, 1876:493. Gemminger \& Harold, 1876:3705. - Kuhnt, 1909:19; 1911:9. - Deelder, 1942:59. - Mader, 1942d:153; 1944:98. - Blackwelder, 1945:457. Guérin, 1948:5. Distr.: Peru. Suriname. Brasil, região amazônica.

hogei Gorham, 1888:89. Tp.: NHML exam. aut. - (México, Guerrero, Chilpancingo). - Kuhnt, 1909:18; 1911:9. - Blackwelder, 1945:457. Distr.: México.

inflatus Crotch, 1876:493. Tp.: MCZC exam. aut. - (Peru, Pebas). - Gemminger \& Harold, 1876:3705. - Kuhnt, 1909:19; 1911:9. - Mader, 1942d:153; 1944:98. - Blackwelder, 1945:457. - Guérin, 1948:5. Distr.: Peru. Brasil, região amazônica.

jansoni Crotch, 1873b:146. Tp.: NHML exam. aut. - (Nicarágua, Chontales, Santo Domingo). - Gemminger \& Harold, 1876:3705. - Gorham, 1888:88, pl. 5, fig. 1. - Kuhnt, 1909:19; 1911:9. - Blackwelder, 1945:457. Distr.: Nicarágua.

lateritius Lacordaire, 1842:293. Tp. ? - (Caiena). - Crotch, 1876:494. - Gemminger \& Harold, 1876:3705. - Kuhnt, 1909:19; 1911:9. - Blackwelder, 1945:457. Distr.: Guiana Francesa. Brasil, região amazônica.

leachi Lacordaire, 1842:287. Tp. ? - (Colômbia). - Crotch, 1876:491. - Gemminger \& Harold, 1876:3705. - Kuhnt, 1909:19; 1911:9. - Blackwelder, 1945:457. Distr.: Colômbia.

lebasi Lacordaire, 1842:285. Tp. ? - (Colômbia, Cartagena). - Crotch, 1876:494.

- Gemminger \& Harold, 1876:3705. - Gorham, 1888:89. - Kuhnt, 1909:18; 1911:9. - Deelder, 1942:59. - Blackwelder, 1945:457. Distr.: Costa Rica, Panamá. Colômbia.

cassideus Lacordaire, 1842:295. - Crotch, 1876:494 (syn.).

lineatus Guérin-Méneville, 1844:308. Tp.: MNHN ex aut. - (Colômbia). - Crotch, 1876:492. - Gemminger \& Harold, 1876:3705. - Kuhnt, 1909:18; 1911:9.

- Blackwelder, 1945:457. Distr.: Colômbia. Brasil, região amazônica.

var. thoracicus Kuhnt, 1908b:628. Tp.: ZMHU exam. aut. - (Colômbia, Antioquia); 1909:18; 1911:9. - Blackwelder, 1945:457. Distr.: Colômbia.

lineola Lacordaire, 1842:294. Tp. ? - (Colômbia). - Crotch, 1876:494. - Gemminger \& Harold, 1876:3705. - Kuhnt, 1909:19; 1911:9. - Blackwelder, 
1945:457. Distr.: Colômbia.

luridus Kuhnt, 1908b:627. Tp.: ZMHU exam. aut. - (Colômbia); 1909:19; 1911:9. - Deelder, 1942:59. - Blackwelder, 1945:457. Distr.: Colômbia. Suriname.

luteus Erichson, 1847:179. Tp.: ZMHU exam. aut. - (Peru). - Crotch, 1876:496. - Gemminger \& Harold, 1876:3705. - Kuhnt, 1909:19; 1911:9. - Deelder, 1942:59. - Mader, 1942d:153; 1944:98. - Blackwelder, 1945:457. Distr.: Peru.

maculicollis Duponchel, 1825:158, pl. 7, fig. 64 (Erotylus). Tp. ? - (Brasil). Lacordaire, 1842:288 (Aegithus). - Crotch, 1876:491. - Gemminger \& Harold, 1876:3705. - Kuhnt, 1909:19; 1911:9. - Blackwelder, 1945:457. - Guérin, 1948:5. Distr.: Brasil.

melaspis Gorham, 1888:85, pl. 4, fig. 22. Tp.: NHML exam. aut. - (México, Presidio). - Kuhnt, 1909:18; 1911:9. - Deelder, 1942:59. - Blackwelder, 1945:457. Distr.: México. Guatemala. Nicarágua.

meridionalis Crotch, 1873:146. Tp. ? - (Nicarágua, Chontales); 1876:493. Gorham, 1888:87, pl. 4, fig. 25. - Kuhnt, 1909:18; 1911:10. - Deelder, 1942:59. - Blackwelder, 1945:457. Distr.: México. América Central.

mesosternalis Kuhnt, 1908b:626. Tp.: ZMHU exam. aut. - (Colômbia); 1909:19; 1911:10. - Blackwelder, 1945:457. Distr.: Colômbia.

monochrous Lacordaire, 1842:294. Tp. ? - (Caiena). - Crotch, 1876:494. Gemminger \& Harold, 1876:3705. - Kuhnt, 1909:19; 1911:10. - Blackwelder, 1945:457. Distr.: Guiana Francesa.

multistriatus Mader, 1942d:153, 177. Tp.: MGFM exam. aut. - (Peru, Chanchamayo); 1944:99. Distr.: Peru. Brasil, região amazônica.

nigrocinctus Erichson, 1847:179. Tp.: ZMHU exam. aut. - (Peru). - Crotch, 1876:495. - Gemminger \& Harold, 1876:3705. - Kuhnt, 1909:19; 1911:10. - Deelder, 1942:59. - Mader, 1942d:153; 1944:98. - Blackwelder, 1945: 457. Distr.: Peru.

olivaceipennis Mader, 1942c:552, 553. Tp., MGFM exam. aut. - (Bolívia, Coroico). Distr.: Bolívia.

ornaticollis Lacordaire, 1842:292. Tp. ? - (Colômbia). - Crotch, 1876:492. Gemminger \& Harold, 1876:3705. - Kuhnt, 1909:19; 1911:10. - Blackwelder, 1945:457. Distr.: Colômbia.

pallidus Kuhnt, 1908b:626. Tp.: ZMHU exam. aut. - (Brasil, Pará, Cametá); 1909;19; 1911:10. - Deelder, 1942:59. - Blackwelder, 1945:457. Distr.: Brasil, região amazônica.

politissimus Crotch, 1876:493. Tp.: MCZC exam. aut. - (Equador). - Gemminger \& Harold, 1876:3705. - Kuhnt, 1909:19; 1911:10. - Blackwelder, 1945: 457. Distr.: Equador.

politus Gorham, 1888:86. Tp.: NHML exam. aut. - (Nicarágua, Chontales). 
Kuhnt, 1909:18; 1911:10. - Blackwelder, 1945:457. Distr.: Nicarágua. Costa Rica. Panamá.

punctatissimus Fabricius, 1775:123 (Erotylus). Tp.: HCGU - (América); 1781: 157; 1787:91; 1792:37; 1801:5. - Olivier, 1792:433; 1807:469, pl. 2, fig. 13. - Illiger, 1806:231. - Gmelin, 1790:1727. - Herbst, 1799:367, pl. 137, fig. 8. - Schönherr, 1808:326. - Duponchel, 1825:157, pl. 7, fig. 61. Zimsen, 1964:114. - Lacordaire, 1842:281 (Aegithus). - Erichson, 1848: 579. - Crotch, 1876:490. - Gemminger \& Harold, 1876:3706. - Kuhnt, 1909:18; 1911:10. - Staig, 1931:102. - Curran, 1941:282. - Deelder, 1942:59. - Mader, 1942d:153; 1944:97. - Blackwelder, 1945:457. Distr.: América do Sul, região amazônica.

centumpunctatus Herbst, 1783:45, pl. 22, fig. 13 (Coccinella). - Lacordaire, 1842:281 (syn.).

separandus Crotch, 1876:490. - Curran, 1941:282 (syn.).

punctata Voet, 1778:44. pl. 33, fig. 3 (Pseudochrysomela). - Alvarenga, 1977:105 (syn.).

quadrimaculatus Gistel, 1848:124. Tp. ? - [Localidade não citada]. Distr.: ?

quadrinotatus Chevrolat, 1834, fasc. 4, Tp. ? - (México, Toulepeck). - Lacordaire, 1842:283. - Guérin-Méneville, 1844:308. - Crotch, 1873b:146; 1876:495. - Gemminger \& Harold, 1876:3706. - Gorham, 1888:89. - Kuhnt, 1909:19; 1911:10. - Deelder, 1942:59. - Blackwelder, 1945:457. - Pallister, 1955b: 5. - Candèze, 1861:398, pl. 6, fig. 3 Distr.: México. Belize. Guatemala. Nicarágua.

clathratus Lacordaire, 1842:282. - Crotch, 1873b:146 ( $\sin )$.

rhombifer Casey, 1916:169. Tp.: NMNH - (Honduras, San Pedro Sula). Blackwelder, 1945:457. Distr.: Honduras.

rubriventris Kuhnt, 1908b:626. Tp.: ZMHU exam. aut. - (Brasil); 1909:19; 1911:10. - Blackwelder, 1945:457. Distr.: Brasil, região sudeste.

rufipennis Chevrolat, 1834, fasc. 4. Tp.: MCZC exam. aut. - (México, Orizaba). - Lacordaire, 1842:284. - Crotch, 1876:492. - Gorham, 1888:87, pl. 4, fig. 24. - Kuhnt, 1909:18; 1911:10. - Deelder, 1942:59. - Blackwelder, 1945:457. - Pallister, 1955b:4. Distr.: México. Belize. Guatemala.

sanguinans Dohrn, 1880:152, 293. Tp. ? - [Localidade não citada]. Fleutiaux, 1886:224. - Kuhnt, 1909:18, pl. 2, fig. 2; 1911:10. - Deelder, 1942:59. Mader, 1942d:151; 1944:96. - Blackwelder, 1945:457. - Guérin, 1948:5. Distr. : Peru. Brasil, região amazônica.

satellitius Lacordaire, 1842:295. Tp. ? - (Colômbia). - Crotch, 1876:495. Gemminger \& Harold, 1876:3706. - Kuhnt, 1909:19; 1911:10. - Mader, 1942d:153; 1944:98. - Blackwelder, 1945:457. Distr.: Colômbia. Peru. Brasil, região amazônica.

schenklingi Mader, 1942c:551. Distr.: Brasil, região amazônica.

clathratus; Schenkling, 1919, nec Lacordaire, 1842. - Schenkling, 1919:79. 
Tp.: DEIB - (Brasil). - Mader, 1942c:551 (nom.praeoc.)

scurra Lacordaire, 1842:288. Tp. ? - (Brasil). - Crotch, 1876:494. - Gemminger \& Harold, 1876:3706. - Kuhnt, 1909:19; 1911:10. - Blackwelder, 1945: 457. Distr.: Brasil, região sudeste.

stillatus Gorham, 1888:91. Tp.: NHML exam. aut. - (México). - Kuhnt, 1909:18; 1911:10. - Blackwelder, 1945:457. Distr.: México. Belize.

striatellus Crotch, 1876:495. Tp. ? - (Equador). - Gemminger \& Harold, 1876: 3706. - Kuhnt, 1909:19; 1911:10. - Schenkling, 1919:79. - Mader, 1942d:153; 1944:98. - Blackwelder, 1945:457. Distr.: Equador. Peru.

strigicollis Gorham, 1888:90. Tp.: NHML exam. aut. -(Panamá, David). - Kuhnt, 1909:19; 1911:10. - Blackwelder, 1945:457. Distr.: Panamá.

suturella Lacordaire, 1842:290. Tp.: ? - (Colômbia). - Crotch, 1876:491. Gemminger \& Harold, 1876:3706. - Kuhnt, 1909:19; 1911:10. - Blackwelder, 1945:457. Distr.: Colômbia.

torquatus Lacordaire, 1842:291. Tp.: MCZC exam. aut. - (Caiena). - Crotch, 1876:494. - Gemminger \& Harold, 1876:3706. - Kuhnt, 1909:19; 1911:10. - Mader, 1942c:552. - Blackwelder, 1945:457. Distr.: Guiana Francesa.

trilineatus Kuhnt, 1908b:626. Tp.: ZMHU exam. aut. - (México); 1909:19; 1911:10. - Blackwelder, 1945:457. Distr.: México.

truncatus Crotch, 1876:492. Tp.: MCZC exam. aut. - (Equador). - Gemminger \& Harold, 1876:3706. - Gorham, 1891:56. - Kuhnt, 1909:18; 1911:11. Blackwelder, 1945:457. Distr.: Equador.

uva Lacordaire, 1842:290. Tp. ? - (Colômbia). - Crotch, 1876:492. - Gemminger \& Harold, 1876:3706. - Gorham, 1888:88; 1898:255. - Kuhnt, 1909:18; 1911:11. - Deelder, 1942:59. - Blackwelder, 1945:457. Distr.: México. América Central. Colômbia. Equador.

var. abdominalis Kuhnt, 1908b:627. Tp. ? - (Costa Rica); 1909:18; 1911:11. - Deelder, 1942:59. - Blackwelder, 1945:457. Distr.: Costa Rica. Colômbia.

var.brunneus Kuhnt, 1908b:628. Tp. ? - (Costa Rica); 1909:18; 1911:11. Blackwelder, 1945:457. Distr.: Costa Rica.

varicollis Lacordaire, 1842:291. Tp.: MCZC exam. aut. - (Colômbia). - Crotch, 1876:491. - Gemminger \& Harold, 1876:3706. - Gorham, 1888:88. Kuhnt, 1909:19; 1911:11. - Schenkling, 1919:79. - Deelder, 1942:59.

Blackwelder, 1945:457. Distr.: Guatemala. Panamá. Colômbia.

var. collaris Kuhnt, 1908b:627. Tp.: ZMHU exam. aut. - (Colômbia); 1909:19; 1911:9. - Mader, 1942c:551. - Blackwelder, 1945:457. Distr.: Colômbia.

walkenaeri Lacordaire, 1842:280. Tp. ? - [Localidade não citada]. - Crotch, 1876: 490. - Fleutiaux, 1886:224. - Kuhnt, 1909:18; 1911:11. - Blackwelder, 1945:457 - Guérin, 1948:5. Distr.: Brasil, região sudeste. 


\section{Iphiclus Chevrolat, 1837}

Iphiclus Chevrolat, 1837:426 (Tgen.: Erotylus flavovittatus Duponchel, 1825:59, seg. Alvarenga, 1965:82). - Alvarenga, 1965:82.

Brachysphoenus Lacordaire, 1842:32, 296. - Crotch, 1873b:147; 1876:496. -

Chapuis, 1876:49, 56. - Mader, 1944:99.

Brachysphaenus Kuhnt, 1909:9, 20. - Deelder, 1942:51.

Brachysphenus Gorham, 1888:92.

Morphoides Gemminger \& Harold, 1876:3706.

\section{Iphiclus (Megaprotus) Lacordaire, 1842}

Megaprotus Lacordaire, 1842:297 (Tsubgen.: Erotylus signatus Duponchel, 1825: 159, seg. Alvarenga, 1965:86). - Chapuis, 1876:58. - Crotch, 1876:496. Kuhnt, 1909:22-23. - Mader, 1944:102. - Alvarenga, 1965:86.

ardens Curran, 1941:284, fig. 5 (Brachysphaenus). Tp.: DTNY - (Guiana, Kartabo). Distr.: Guiana.

argus Kuhnt, 1910:257, fig. 17 (Brachysphaenus). Tp.: ZMHU exam. aut. (Brasil, Bahia); 1911:12. - Blackwelder, 1945:458. Distr.: Brasil, regiões nordeste e sudeste.

catillifer Gorham, 1888:93, pl. 5, figs 10, 11 (Brachysphenus). Tp.: NHML exam. aut. - (Nicaragua, Chontales, Santo Domingo). - Kuhnt, 1909:24; 1911:13.

- Blackwelder, 1945:458. Distr.: Panamá. Costa Rica.

cinctellus Lacordaire, 1842:300 (Brachysphoenus). Tp. ? - (Caiena). - Crotch, 1876:496. - Gemminger \& Harold, 1876:3707. - Kuhnt, 1909:24; 1911:13.

- Blackwelder, 1945:458. Distr.: Guiana Francesa. Brasil, região amazônica.

circulus Lacordaire, 1842:307 (Brachysphoenus). Tp. ? - (Caiena). - Crotch, 1876:498. - Kirsch, 1876:102. - Gemminger \& Harold, 1876:3707. Kuhnt, 1909:24; 1911:13. - Mader, 1942d:157; 1944:103. - Blackwelder, 1945:458. Distr.: Guiana Francesa. Peru.

clarosignatus Kuhnt, 1910:258 (Brachysphaenus). Tp.: ZMHU exam. aut. (Guiana Francesa, Caiena); 1911:13. - Deelder, 1942:60. - Blackwelder, 1945:458. Distr.: Suriname. Guiana Francesa.

coadunatus Lacordaire, 1842:303 (Brachysphoenus). Tp. ? - (Caiena). - Crotch, 1876:499. - Gemminger \& Harold, 1876:3707. - Kuhnt, 1909:23; 1911:13.

- Blackwelder, 1945:458. Distr.: Guiana Francesa. Brasil, região amazônica.

cunctabundus Mader, 1942c:553 (Brachysphoenus). Tp.: MGFM exam. aut. (Bolívia, Coroico). Distr.: Bolívia.

decussatus Lacordaire, 1842:298 (Brachysphoenus). Tp. ? -(Colômbia). - Crotch, 
1876:496. - Gemminger \& Harold, 1876:3708. - Kuhnt, 1909:24; 1911:14.

- Blackwelder, 1945:458. Distr.: Colômbia.

delineatus Lacordaire, 1842:306 (Brachysphoenus). Tp. ? - (Caiena). - Crotch, 1876:497. - Gemminger \& Harold, 1876:3708. - Kuhnt, 1909:24; 1911:14.

- Deelder, 1942:61. - Blackwelder, 1945:458. Distr.: Panamá. Colômbia. Guiana Francesa. Brasil, região amazônica.

dilectus Gorham, 1888:93 (Brachysphenus). Tp.: NHML exam. aut. - (Panamá, volcán de Chiriquí), - Kuhnt, 1909:23; 1911:14. - Blackwelder, 1945:458.

Distr.: Panamá. Peru.

duodecimpustulatus Lacordaire, 1842:301 (Brachysphoenus). Tp. ? - (Caiena). Crotch, 1876:496. - Gemminger \& Harold, 1876:3708. - Kuhnt, 1909:24; 1910:260; 1911:14. - Blackwelder, 1945:458. Distr.: Guiana Francesa. Brasil, região amazônica.

duplicatus Lacordaire, 1842:299 (Brachysphoenus). Tp.: MCZC exam. aut. (Colômbia). - Guérin-Méneville, 1855:611. - Crotch, 1876:498. - Gemminger \& Harold, 1876:3708. - Kuhnt, 1909:23; 1911:14. - Deelder, 1942:61. - Mader, 1942d:157; 1944:103. - Blackwelder, 1945:458. Guérin, 1948:13. Distr.: Colômbia. Peru. Brasil, região central. var. mediatus Lacordaire, 1842:308. Tp.: MCZC exam. aut. - (Colômbia). Crotch, 1876:498. - Gemminger \& Harold, 1876:3708. - Kuhnt, 1909:23; 1911:14. - Mader, 1942d:157. - Blackwelder, 1945:458. Distr.: Colômbia.

ephippium Duponchel, 1825:163, pl. 7, fig. 77 (Erotylus). Tp. ? (Guiana Francesa). - Lacordaire, 1842:302 (Brachysphoenus). - Crotch, 1876:497. Gemminger \& Harold, 1876:3708. - Kuhnt, 1909:24, pl. 2, fig. 5; 1911:14. - Bruch, 1914:380. - Deelder, 1942:61. - Blackwelder, 1945:458. - Guérin, 1948:13. Distr.: Guiana Francesa?. Brasil, regiões sudeste e sul. Argentina.

epigraphus Crotch, 1876:500 (Brachysphoenus). Tp.: MCZC exam. aut. - (Amazonas). - Gemminger \& Harold, 1876:3708. - Kuhnt, 1909:23; 1911:15. Blackwelder, 1945:458. Distr.: Brasil, região amazônica.

eximius Lacordaire, 1842:393 (Brachysphoenus). Tp. ? - (Brasil). - Crotch, 1876:499. - Gemminger \& Harold, 1876:3708. - Kuhnt, 1909:24; 1911:15. - Blackwelder, 1945:458. Distr.: Brasil.

fasciellus Crotch, 1876:497 (Brachysphoenus). Tp.: MCZC exam. aut. - (Amazonas). - Gemminger \& Harold, 1876:3708. - Kuhnt, 1909:24; 1911:15. Blackwelder, 1945:458. Distr.: Brasil, região amazônica.

fasciunculus Crotch, 1876:497 (Brachysphoenus)_. Tp.: MCZC exam. aut. (Amazonas). - Gemminger \& Harold, 1876:3708. - Kuhnt, 1909:24; 1911:15. - Blackwelder, 1945:458. Distr.; Brasil, região amazônica.

laetus Crotch, 1876:499 (Brachysphoenus). Tp.: MCZC exam. aut. - (Amazonas). - Gemminger \& Harold, 1876:3709. - Kuhnt, 1909:23; 1911:16. - Mader, 
1942d:157; 1944:102. - Blackwelder, 1945:459. Distr.: Peru. Brasil, região amazônica.

ab. mediolineatus Mader, 1942d:157. Tp.: DEIB - (Peru); 1944:102. Distr.:

Peru. Brasil, região amazônica.

laevipennis Kuhnt, 1910:256 (Brachysphaenus). Tp. ? - (Guiana Francesa, Caiena); 1911:16. - Blackwelder, 1945:459. Distr.: Guiana Francesa.

luctuosus Kuhnt, 1910:260 (Brachysphaenus). Tp.: ZMHU exam. aut. - (Brasil, Mato Grosso); 1911:16. - Blackwelder, 1945:459. Distr.: Brasil, região central.

luteoniger Crotch, 1876:498 (Brachysphoenus). Tp. ? - (Equador). - Gemminger \& Harold, 1876:3709. - Kuhnt, 1909:23; 1911:16. - Blackwelder, 1945: 459. Distr.: Equador.

luteopictus Crotch, 1876:501 (Brachysphoenus). TP.: MCZC exam. aut. - (Amazonas). - Gemminger \& Harold, 1876:3709. - Kuhnt, 1909:23; 1911:16. Blackwelder, 1945:459. - Guérin, 1948:6. Distr.: Brasil, região amazônica.

masculinus Crotch, 1876:499 (Brachysphoenus). Tp.: MCZC exam. aut. - (Amazonas). - Gemminger \& Harold, 1876:3710. - Kuhnt, 1909:23; 1911:17. Blackwelder, 1945:459. Distr.: Brasil, região amazônica.

moniliferus Guérin-Méneville, 1841 b: 155 (Mycotretus). Tp.: MCZC exam. aut. - (Guiana Francesa, Caiena); 1855:611 (Brachysphaenus). - Lacordaire, 1842:302. - Crotch, 1876:496. - Gemminger \& Harold, 1876:3710. Kuhnt, 1909:24; 1911:17. - Curran, 1941:284. - Blackwelder, 1945:459.

Distr.: Guiana. Guiana Francesa.

nubilus Lacordaire, 1842:310 (Brachysphoenus). Tp. ? - (Caiena). - Crotch, 1876:499. - Gemminger \& Harold, 1876:3710. - Kuhnt, 1909:23; 1911:17.

- Blackwelder, 1945:459. Distr.: Guiana Francesa.

nuculus Gorham, 1888:94 (Brachysphenus). Tp.: NHML exam. aut. - (México,

Toxpam). - Kuhnt, 1909:23; 1911:17. - Blackwelder, 1945:459. Distr.:

México.

obliteratus Guérin, 1956:57, fïg. 19 (Brachysphaenus). Tp.: CDSP exam. aut. -

(Brasil, Est. Rio de Janeiro, Itatiaia). Distr.: Brasil, região sudeste.

octolinearis Kuhnt, 1910:257, fig. 16 (Brachysphaenus). Tp.: ZMHU exam. aut. - (Peru, Chanchamayo); 1911:18. - Mader, 1942d:157; 1944:104. - Blackwelder, 1945:459. Distr.: Peru.

octopunctatus Kirsch, 1876:103 (Megaprotus). Tp.: SMTD exam. aut. - (Peru, Pozuzo). - Gemminger \& Harold, 1876:3710. - Kuhnt, 1909:24 (Brachysphaenus); 1911:18. - Mader, 1942d:158; 1944:104. - Blackwelder, 1945:459. - Delkeskamp, 1957:96. Distr.: Peru. Equador. Bolívia.

orphanulus Lacordaire, 1842:307 (Brachysphoenus). Tp. ? - (Caiena). - Crotch, 1876:500. - Gemminger \& Harold, 1876:3710. - Kuhnt, 1909:23; 1911:18.

- Blackwelder, 1945:459. Distr.: Guiana Francesa. 
patruelis Kuhnt, 1910:259, fig.18 (Brachysphaenus). Tp.: ZMHU exam. aut. (Brasil); 1911:18. - Blackelder, 1945:459. Distr.: Brasil.

perlepidus Lacordaire, 1842:299 (Brachysphoenus). Tp.: MNHN exam. aut. (Caiena). - Blanchard, 1843, pl. 25, fig. 8. - Crotch, 1876:497. - Gemminger \& Harold, 1876:3710. - Kuhnt, 1909:24; 1911:18. - Mader, 1942d:157; 1944:103. - Blackwelder, 1945:459. Distr.: Guiana Francesa. Peru. Bolívia.

pithecius Lacordaire, 1842:309 (Brachysphoenus). Tp. ? - (Caiena). Crotch, 1876:498. - Gemminger \& Harold, 1876:3710. - Kuhnt, 1909:23; 1911:18.

- Blackwelder, 1945:459. Distr.: Guiana Francesa.

porcellana Lacordaire, 1842:300 (Brachysphoenus). Tp. ? - (Caiena). - Crotch, 1876:500. - Gemminger \& Harold, 1876:3711. - Kuhnt, 1909:23; 1911:18.

- Blackwelder, 1945:459. Distr.: Guiana Francesa.

pulcher Gorham, 1888:93, pl. 5, fig. 9 (Brachysphenus). Tp.: NHML - (Panamá, Bugaba, volcán Chiriquí). - Kuhnt, 1909:24; 1911:18. - Blackwelder, 1945:459. Distr.: Panamá.

sedecimlinearis Mader, 1942d:157, 183 (Brachysphoenus). Tp.: SMTD (Peru); 1944:104. Distr.: Peru.

sedecimpunctatus Lacordaire, 1842:309 (Brachysphoenus). Tp. ? - (Caiena). Crotch, 1876:498. - Gemminger \& Harold, 1876:3711. - Kuhnt, 1909:24; 1911:19. - Blackwelder, 1945:460. Distr.: Guiana Francesa.

sedecimstrigatus Mader, 1942d:157, 182 (Brachysphoenus). Tp.: MGFM exam. aut. - (Peru, Chanchamayo); 1944:103. Distr.: Peru.

sexsigillatus Lacordaire, 1842:390 (Brachysphoenus). Tp. ? - (Brasil). - Crotch, 1876:499. - Gemminger \& Harold, 1876:3711. - Kuhnt, 1909:24; 1911:19. - Blackwelder, 1945:460. Distr.: Brasil.

signatus Duponchel, 1825:159, pl. 7, fig. 67 (Erotylus). Tp. ? - (Brasil). Lacordaire, 1842:304 (Brachysphoenus). - Crotch, 1876:497. - Gemminger \& Harold, 1876:3711. - Kuhnt, 1909:24; 1911:20. - Schenkling, 1919:80. - Deelder, 1942:63. - Mader, 1942c:554. - Blackwelder, 1945: 460. - Guérin, 1948:13. Distr.: Brasil, regiões sudeste e sul.

amabilis Guérin-Méneville, 1841:154 (Brachymerus). - Lacordaire, 1842:305 (Brachysphoenus). - Mader, 1942c:554 (syn.).

zonulus Crotch, 1873b:147 (Brachysphoenus). Tp.: MCZC exam. aut. - (Equador); 1876:498. - Gemminger \& Harold, 1876:3712. - Kuhnt, 1909:24; 1911:20. - Mader, 1942d:157; 1944:103. - Blackwelder, 1945:460. Distr.: Nicaraguá. Equador. Peru. Brasil, região amazônica. 


\section{Iphiclus (Habrodactylus) Lacordaire, 1842}

Habrodactylus Lacordaire, 1842: 311 (Tsubgen.: Brachysphoenus sulphurifer Lacordaire, 1842: 324, seg. Alvarenga, 1965:84). - Chapuis, 1876:58. Crotch, 1876:503. - Kuhnt, 1909:22, 24. - Mader, 1944:104. - Alvarenga, 1965:84.

agathinus Guérin-Méneville, 184 lb:154 (Brachymerus). Tp.: MNHN exam. aut. - (Brasil). - Lacordaire, 1842:411 (Brachysphoenus). - Crotch, 1876:508. - Gemıninger \& Harold, 1876:3707. - Kuhnt, 1909:25; 1911:12. - Blackwelder, 1945:457. Distr.: Brasil, região sudeste.

annulatus Germar, 1824:613 (Erotylus). - Tp.: ZMHU exam. aut. - (Brasil). Sturm, 1826:139. - Lacordaire, 1842:330 (Brachysphoenus). - Crotch, 1876:500. - Gemminger \& Harold, 1876:3707. - Kuhnt, 1909:23; 1911:12. - Blackwelder, 1945:458. - Guérin, 1948:10. Distr.: Brasil, região sudeste e sul.

antennalis Lacordaire, 1842:410 (Brachysphoenus). Tp. ? - (Caiena). - Crotch, 1876:507. - Gemminger \& Harold, 1876:3707. - Kuhnt, 1909:24; 1911:12. - Blackwelder, 1945:458. Distr.: Guiana Francesa.

arculifer Kirsch, 1883:211 (Brachysphoenus). Tp.: SMTD exam. aut. - (Equador). - Fleutiaux, 1886:224. - Kuhnt, 1909:25; 1911:12. - Blackwelder, 1945: 458. DIstr.: Equador.

basinotatus Crotch, 1876:504 (Brachysphoenus). Tp.: MCZC exam. aut. - (Brasil). - Gemminger \& Harold, 1876:3707. - Kuhnt, 1909:25; 1911:12. Blackwelder, 1945:458. Distr.: Brasil, região sudeste.

bisquadripunctatus Kuhnt, 1911:12 (Brachysphaenus). - Blackwelder, 1945:458. - Guérin, 1946b:370; 1948:13. Distr.: Brasil.

octopunctatus; Kuhnt, 1910, nec Kirsch, 1876. - Kuhnt, 1910:263, fig. 20. Tp.: ZMHU exam. aut. - (Brasil); 1911:12. - Guérin, 1946b:370 (nom. praeoc.).

kirschi Kuhnt, 1911:16. - Blackwelder, 1945:459. - Guérin, 1946b:370 (syn.). bisquinquepunctatus Lacordaire, 1842:318 (Brachysphoenus). Tp. ? - (Caiena). Crotch, 1876:504. - Gemminger \& Harold, 1876:3707. - Kuhnt, 1909:25; 1910:265; 1911:13. - Mader, 1943:110. - Blackwelder, 1945:458. Distr.: Guiana Francesa.

bisquinquesignatus Mader, 1943:110 (Brachysphoenus). Tp. ? - (Brasil, Est. São Paulo, Santos). Distr.: Brasil, região sudeste.

bistripunctatus Lacordaire, 1842:314 (Brachysphoenus). Tp.: MCZC exam. aut. - (Caiena). - Crotch, 1876:503. - Gemminger \& Harold, 1876:3707. Kuhnt, 1909:25; 1911:13. - Blackwelder, 1945:458. Distr.: Guiana Francesa.

brevicollis Gorham, 1888:95 (Brachysphenus). Tp.: NHML - (Panamá, Bugaba). - Kuhnt, 1909:24; 1911:13. - Blackwelder, 1945:458. Distr.: Panamá. 
centromaculatus Lacordaire, 1842:329 (Brachysphoenus). Tp.: MCZC exam. aut. - (Caiena). - Crotch, 1876:508. - Gemminger \& Harold, 1876:3707. Kuhnt, 1909:25; 1911:13. - Blackwelder, 1945:458. Distr.: Guiana Francesa.

cereus Gorham, 1888:94 (Brachysphenus). Tp.: NHML exam. aut. - (Panamá, Bugaba, volcán Chiriquí). - Kuhnt, 1909:24; 1911:13. - Deelder, 1942:60. - Blackwelder, 1945:458. Distr.: Panamá.

concolor Lacordaire, 1842:328 (Brachysphöenus). Tp. ? - (Caiena). - Crotch, 1876:507. - Gemminger \& Harold, 1876:3708. - Kuhnt, 1909:24; 1911:13.

- Blackwelder, 1945:458. Distr.: Guiana Francesa.

congener Lacordaire, 1842:318 (Brachysphoenus). Tp. ?.(Rio de Janeiro).

Crotch, 1876:504. - Gemminger \& Harold, 1876:3708. - Kuhnt, 1909:24; 1911:13. - Blackwelder, 1945:458. - Guérin, 1948:13. Distr.: Brasil, regiões sudeste e sul.

conspicillatus Gorham, 1888:97, pl. 5, fig. 12 (Brachysphenus). Tp.: NHML exam. aut. - (Costa Rica, Cachí). - Kuhnt, 1909:25; 1911:14. - Blackwelder, 1945:458. Distr.: Costa Rica.

cordiger Crotch, 1876:507 (Brachysphoenus). Tp.: MCZC exam. aut. - (Ega) [atualmente Tefé]. - Gemminger \& Harold, 1876:3708. - Kuhnt, 1909:25; 1911:14. - Blackwelder, 1945:458. Distr.: Brasil, região amazônica.

decemoculatus Mader, 1943:110 (Brachysphoenus). Tp. ? - (Brasil, Santa Catarina, Mafra). Distr.: Brasil, região sul.

decemplagiatus Kuhnt, 1910:264 (Brachysphaenus). Tp.: ZMHU exam. aut. (Brasil); 1911:14. - Mader, 1943:110. - Blackwelder, 1945:458. Distr.: Brasil.

decempunctatus Duponchel, 1825:162, pl. 7, fig. 74 (Erotylus). Tp.: MNHN exam. aut. - (Brasil). - Lacordaire, 1842:317 (Brachysphoenus). - Crotch, 1876:504. - Gemminger \& Harold, 1876:3708. - Kuhnt, 1909:25; 1910: 265; 1911:14. - Bruch, 1914:380. - Mader, 1943:110. - Blackwelder, 1945:458. - Guérin, 1948:13. Distr.: Brasil, região sudeste.

var. quadristictus Guérin, 1956:46. Tp.: CDSP exam. aut. - (Brasil, Est. S.

Paulo, S. Paulo, Casa Grande). Distr.: Brasil, região sudeste.

deficiens Crotch, 1876:505 (Brachysphoenus). Tp.: MCZC exam. aut. - (Amazonas). - Fleutiaux, 1886:224. - Kuhnt, 1909:25; 1911:14. - Blackwelder, 1945:458. - Distr.: Brasil, região amazônica.

deletus Lacordaire, 1842:408 (Brachysphoenus). Tp. ? - (Caiena). Crotch, 1876: 508. - Gemminger \& Harold, 1876:3708. - Kuhnt, 1909:25; 1911:14. Blackwelder, 1945:458. Distr.: Guiana Francesa.

detritus Lacordaire, 1842:314 (Brachysphoenus). Tp.: MCZC exam. aut. - (Colômbia). - Crotch, 1876:504. - Gemminger \& Harold, 1876:3708. - Kuhnt, 1909:25; 1911:14. - Deelder, 1942:61. - Blackwelder, 1945:458. Distr.: Colômbia. 
discrepans Guérin, 1956:46, fig. 3 (Brachysphaenus). Tp.: CDSP exam. aut. (Brasil, Santa Catarina, Nova Teutônia). Distr.: Brasil, região sul.

discus Lacordaire, 1842:327 (Brachysphoenus). Tp. ? - (Caiena). - Crotch, 1876:507. - Gemminger \& Harold, 1876:3708. - Kuhnt, 1909:25; 1911:14. - Blackwelder, 1945:458. Distr.: Guiana Francesa.

egensis Gorham, 1889:615, pl. 61, fig. 8 (Brachysphenus) Tp.: NHML exam. aut. - (Brasil, Amazonas, Ega)[atualmente Tefé]. - Kuhnt, 1909:25; 1911: 14. - Blackwelder, 1945:458. Distr.: Brasil, região amazônica.

exiguenotatus Gorham, 1888:95 (Brachysphenus). Tp.: NHML - (Nicarágua). Kuhnt, 1909:15. - Blackwelder, 1945:458. Distr.: Nicarágua. Panamá.

festivus Lacordaire, 1842:332 (Brachysphoenus). Tp. ? - (México). - Crotch, 1876:555 (Priotelus). - Gorham, 1888:100, pl. 5, fig. 14 (Brachysphenus). - Gemminger \& Harold, 1876:3708. - Kuhnt, 1909:25; 1911:15. - Blackwelder, 1945:458. Distr.: México.

fulvipennis Erichson, 1847:179 (Brachysphenus). Tp. ZMHU exam. aut. - (Peru). - Crotch, 1876:522. - Gemminger \& Harold, 1876:3709. - Kuhnt, 1909:25; 1911:15. - Deelder, 1942:62. - Mader, 1942d:158; 1944:105. - Blackwelder, 1945:458. Distr.: Equador. Peru.

fuscipes Duponchel, 1825:160, pl. 7, fig. 69 (Erotylus). Tp. ? - (Brasil). Lacordaire, 1842:410 (Brachysphoenus). - Crotch, 1876:507. - Gemminger \& Harold, 1876:3709. - Kuhnt, 1909:24; 1911:15. - Deelder, 1942:62. - Blackwelder, 1945:458. Distr.: Brasil, região sudeste.

fuscomaculatus Duponchel, 1825:57, pl. 2, fig. 50 (Erotylus). Tp. ? - (Brasil). Lacordaire, 1842:322 (Brachysphoenus). - Crotch, 1876:506. - Gemminger \& Harold, 1876:3709. - Kuhnt, 1909:25; 1911:15. - Blackwelder, 1945:458. Distr.: Brasil, região sudeste.

grammicoides Mader, 1943:111 (Brachysphaenus). Tp.: MGFM exam. aut. (Venezuela). Distr.: Venezuela.

grammicus Erichson, 1847:179 (Brachysphenus). Tр.: ZMHU exam. aut. (Peru). - Crotch, 1876:523. - Gemminger \& Harold, 1876:3709. - Kuhnt, 1909:25; 1911:15. - Mader, 1942d:158; 1944:105. - Blackwelder, 1945: 458. - Delkeskamp, 1957:96. Distr.: Peru. Bolívia.

haematites Lacordaire, 1842:326 (Brachysphoenus). Tp. ? - (Caiena). - Crotch, 1876:507. - Gemminger \& Harold, 1876:3709. - Kuhnt, 1909:24; 1911:15. - Blackwelder, 1945:458. Distrib.: Guiana Francesa.

haematomelas Lacordaire, 1842:320 (Brachysphoenus). Tp. ? - [Localidade não citada]. - Crotch, 1876:506. - Gemminger \& Harold, 1876:4709. - Kuhnt, 1909:25; 1911:16. - Blackwelder, 1945:459. Distr.: Panamá. Colômbia. Bolívia. Argentina.

hamatus Guérin, 1956:47, fïg. 4 (Brachysphaenus). Tp.: CDSP exam. aut. (Brasil, Amazonas, Maués). Distr.: Brasil, região amazônica. 
hoffinanni Lacordaire, 1842:323 (Brachysphoenus). Tp.: MCZC exam. aut. (Brasil). - Crotch, 1876:506. - Gemminger \& Harold, 1876:3709. - Kuhnt, 1909:25; 1911:16. - Blackwelder, 1945:459. - Guérin, 1948:13. Distr.: Brasil, regiões sudeste e sul.

var. reductus Guérin, 1956:47. Tp.: CDSP exam. aut. - (Brasil, Rio Grande do SUl, Serro Azul). Distr.: Brasil, região sul.

hybridus Lacordaire, 1842:319 (Brachysphoenus). Tp. ? - (Rio de Janeiro).

Crotch, 1876:506. - Gemminger \& Harold, 1876:3709. - Kuhnt, 1909:24; 1911:16. - Blackwelder, 1945:459. Distr.: Brasil, região sudeste.

ictericus Lacordaire, 1842:327 (Brachysphoenus). Tp. ? - (Caiena). - Crotch, 1876:507. - Gemminger \& Harold, 1876:3709. - Kuhnt, 1909:24; 1911:16. - Blackwelder, 1945:459. Distr.: Guiana Francesa.

jejunus Gorham, 1888:97 (Brachysphenus). Tp.: NHML - (México, Toxpam). Kuhnt, 1909:24; 1911:16. - Blackwelder, 1945:459. Distr.: México.

kourouensis Lacordaire, 1842:325 (Brachysphoenus). Tp. ? - (Caiena, rio Kourou). - Crotch, 1876:506. - Gemminger \& Harold, 1876:3709. - Kuhnt, 1909:25; 1911:16. Blackwelder, 1945:459. Distr.: Guiana Francesa

manicatus Lacordaire, 1842:312 (Brachysphoenus). Tp. ? - (Colômbia). - Crotch, 1876:500. - Gemminger \& Harold, 1876:3709. - Kuhnt, 1909:23; 1911:16. - Blackwelder, 1945:459. Distr. Colômbia.

melanostictus Crotch, 1876:505 (Brachysphoenus). Tp.: MCZC exam. aut. (Equador). - Gemminger \& Harold, 1876:3710. - Kuhnt, 1909:26; 1911: 17. - Blackwelder, 1945:459. Distr.: Equador.

meleagris Lacordaire, 1842:320 (Brachysphoenus). Tp. ? - (Caiena). - Crotch, 1876:504. - Gemminger \& Harold, 1876:3710. - Kuhnt, 1909:25; 1911:17. - Blackwelder, 1945:459. Distr.: Guiana Francesa.

mendax Lacordaire, 1842:321 (Brachysphoenus). Tp. ? - (Rio de Janeiro). Crotch, 1876:505. - Gemminger \& Harold, 1876:3710. - Kuhnt, 1909:25; 1911:17. - Blackwelder, 1945:459. Distr.: Brasil, região sudeste. litigiosus Lacordaire, 1842:322. - Crotch, 1876:505 (syn.). var. niger Guérin, 1956:47. Tp.: FIOC exam. aut. - (Brasil, Est. Rio de Janeiro, Itatiaia). Distr.: Brasil, região sudeste.

multiguttatus Gorham, 1888:100, pl. 5, fig. 15 (Brachysphoenus). Tp.: NHML (México, Tabasco, Teapa). - Kuhnt, 1909:25; 1911:17. - Blackwelder, 1945:459. Distr.: México. Guatemala.

obliqueguttatus Crotch, 1876:505 (Brachysphoenus). Tp.: MCZC exam. aut. (Brasil). - Gemminger \& Harold, 1876:3710. - Kuhnt, 1909:25; 1911:17. - Blackwelder, 1945:459. - Gérin, 1948:13. Distr.: Brasil, região sul.

oblitus Lacordaire, 1842:325 (Brachysphoenus). Tp. ? - (México). - Crotch, 1876:506. - Gemminger \& Harold, 1876:3710. - Gorham, 1888:101. Kuhnt, 1909:25; 1911:17. Blackwelder, 1945:459. Distr.: México. 
oblongonotatus Lacordaire, 1842:315 (Brachysphoenus). Tp.: MCZC exam. aut. - (Colômbia). - Crotch, 1876:504. - Gemminger \& Harold, 1876:3710. Kuhnt, 1909:25; 1911:17. - Deelder, 1942:62. - Mader, 1942d:158; 1944: 105. - Blackwelder, 1945:459. Distr.: Colômbia. Peru. Brasil, região amazônica.

oliquatus Kuhnt, 1910:262 (Brachysphaenus). Tp.: ZMHU exam. aut. - (Peru); 1911:17. - Mader, 1942d:158; 1944:105. - Blackwelder, 1945:459. Distr.: Peru.

pallidipennis Gorham, 1888:96 (Brachysphenus). Tp.: NHML - (Panamá, Bugaba). - Kuhnt, 1909:24; 1911:18. - Blackwelder, 1945:459. Distr.: Panamá.

palmatus Lacordaire, 1842:347 (Brachysphoenus). Tp.: MCZC exam. aut. (Colômbia). - Crotch, 1876:506. - Gemminger \& Harold, 1876:3710. Kuhnt, 1909:25; 1911:18. - Blackwelder, 1945:459. Distr.: Colômbia. perspicillatus Lacordaie, 1842:312 (Brachysphoenus). Tp.: MCZC exam. aut. (Colômbia). - Crotch, 1876:503. - Gemminger \& Harold, 1876:3710. Kuhnt, 1909:25; 1911:18. - Blackwelder, 1945:459. Distr.: Colômbia.

proximus Guérin-Méneville, 1844:311 (Brachymerus). Tp. ? - (Colômbia). Crotch, 1876:523 (Brachysphoenus). - Gemminger \& Harold, 1876:3711. - Kuhnt, 1909:24; 1911:18. - Mader, 1942d:158; 1944:104. - Blackwelder, 1945:459. Distr.: Colômbia. Peru.

punctiger Lacordaire, 1842:328 (Brachysphoenus). Tp. ? - (Caiena). - Crotch, 1876:507. Gemminger \& Harold, 1876:3711. - Kuhnt, 1909:25; 1911:18. - Blackwelder, 1945:459. Distr.: Guiana Francesa.

quadrimaculatus Duponchel, 1825:58, pl. 2, fig. 52 (Erotylus). Tp. ? - (Brasil) Lacordaire, 1842:313 (Brachysphoenus). - Crotch, 1876:503. - Gemminger \& Harold, 1876:3711. - Kuhnt, 1909:25; 1911:18. - Blackwelder, 1945:459. Distr.: Brasil, região sudeste.

rufescens Lacordaire, 1842:319 (Brachysphoenus). Tp. ? - (Caiena). - Crotch, 1876:506. - Gemminger \& Harold, 1876:3711. - Kuhnt, 1909:24; 1911:19. - Deelder, 1942:63. - Mader, 1942d:158; 1944:104. - Blackwelder, 1945: 459. Distr.: Guiana Francesa. Peru.

rufifrons Lacordaire, 1842:409 (Brachysphoenus). Tp. ? - (Colômbia). - Crotch, 1876:508. - Gemminger \& Harold, 1876:3711. - Kuhnt, 1909:25; 1911:19. - Mader, 1942d:158; 1944:105. - Blackwelder, 1945:459. Distr.: Colômbia. Peru.

scutulatus Kuhnt, 1910:264-265, fig. 21 (Brachysphaenus). Tp.: ZMHU exam. aut. - (Brasil); 1911:19. - Mader, 1943:110. - Blackwelder, 1945:460. Guérin, 1948:13. Distr.: Brasil, região sudeste.

septemmaculatus Guérin, 1956:48, fig. 5 (Brachysphaenus). Tp.: CDSP exam. aut. - (Bolívia, Chapare). Distr.: Bolívia.

spadiceus Lacordaire, 1842:409 (Brachysphoenus). Tp. ? - (Caiena). - Crotch, 
1876:507. - Gemminger \& Harold, 1876:3711. - Kuhnt, 1909:24; 1911:20. - Blackwelder, 1945:460. - Guérin, 1948:13. Distr.: Guiana Francesa. Brasil, região amazônica.

striatipennis Gorham, 1888:96 (Brachysphenus). Tp.: NHML - (Panamá, Bugaba). - Kuhnt, 1909:24; 1911:20. - Blackwelder, 1945:460. Distr.: Panamá.

subsignatus Lacordaire, 1842:329 (Brachysphoenus). Tp. ? - (Caiena). - Crotch, 1876:508. - Gemminger \& Harold, 1876:3711. - Kuhnt, 1909:25; 1911:20, Blackwelder, 1945:460. Distr. Guiana Francesa.

sulphurifer Lacordaire, 1842:324 (Brachysphoenus). - Tp. ? - (Brasil). - Crotch, 1876:506. - Gemminger \& Harold, 1876:3712. - Kuhnt, 1909:26; 1911:20. - Schenkling, 1919:80. - Blackwelder, 1945:460. - Guérin, 1948:13. Distr.: Brasil, regiões sudeste e sul.

tabidus Erichson, 1847:179 (Brachysphenus). Tp. ? - (Peru). - Crotch, 1876:523. - Gemminger \& Harold, 1876:3712. - Kuhnt, 1909:24; 1911:20. - Mader, 1942d: 158; 1944: 104. - Blackwelder, 1945:460. Distr.: Peru.

tetrastictus Crotch, 1876:505 (Brachysphoenus). Tp.: MCZC exam. aut. - (Amazonas). - Gemminger \& Harold, 1876:3712. - Kuhnt, 1909:25; 1911:20. Blackwelder, 1945:460. Distr.: Brasil, região amazônica.

thoracicus Kirsch, 1876:103 (Habrodactylus). Tp.: SMTD exam. aut. Peru. Germming \& Harold, 1876:3712. - Kuhnt, 1911:20 (Brachysphaenus). Mader, 1942d:158; 1944:104. - Blackwelder, 1945:460. Distr.: Peru.

vetula Lacordaire, 1842:316 (Brachysphoenus). Tp.: MNHN exam. aut. - (Caiena). - Crotch, 1876:504. - Gemminger \& Harold, 1876:3712. - Kuhnt, 1909:25; 1911:20. - Blackwelder, 1945:460. Distr.: Guiana Francesa.

\section{Iphiclus (Neacronotus) Alvarenga, 1965}

Neacronotus Alvarenga, 1965:87 (Tsubgen.: Erotylus anmularis Laporte, 1840: 520, desig. orig.).

Acronotus; Lacordaire, 1842, nec Smith, 1827. - Lacordaire, 1842:332 (Brachysphoenus). - Chapuis, 1876:58. - Crotch, 1876:508. - Kuhnt, 1909:22, 26. - Alvarenga, 1965:81 (nom.praeoc.).

annularis Laporte, 1840:520 (Erotylus). Tp. ? - (Colômbia). - Lacordaire, 1842:333 (Brachysphoenus). - Guérin-Méneville, 1855:611. - Crotch, 1876:508. - Gemminger \& Harold, 1876:3707. - Kuhnt, 1909:26; 1911:12. - Deelder, 1942:60. - Blackwelder, 1945:458. Distr.: México. Colômbia. circumdatus Guérin-Méneville, $1841 \mathrm{a} 119$ (Alloiotelus). - Lacordaire, 1842: 333 (syn.).

bimaculicollis Mader, 1942c:554 (Brachysphoenus). Tp.: DEIB - (Colômbia, Cañca). Distr.: Colômbia. 
colombi Guérin-Méneville, $1844: 311$ (Brachysphoenus). Tp.: MNHN exam. aut.

- (Colômbia). - Crotch, 1876:508. - Gemminger \& Harold, 1876:3707. Kuhnt, 1909:26; 1911:13. - Blackwelder, 1945:458. Distr.: Colômbia.

perversus Gorham, 1889:618 (Brachysphenus). Tp.: NHML exam. aut. - (Colômbia, Medellín). - Kuhnt, 1909:26; 1911:18. - Blackwelder, 1945:459. Distr.: Colômbia.

\section{Iphiclus (Sternolobus) Guérin-Méneville, 1841}

Sternolobus Guérin-Méneville, 1841 a:118 (Tsubgen.: Sternolobus bisignatus Guérin-Méneville, 1841a:118. seg. Alvarenga, 1965:89). - Lacordaire, 1842:333. - Chapuis, 1876:59. - Crotch, 1876:508. - Kuhnt, 1909:22, 26. - Mader, 1944:105. - Alvarenga, 1965:89.

atricaudatus Kuhnt, 1910:256, fig. 15 (Brachysphaenus). Tp.: ZMHU exam. aut. - (Equador, Los Llanos); 1911:12. - Blackwelder, 1945:458. Distr.: Equador.

bisignatus Guérin-Méneville, 1841 a:118 (Sternolobus). Tp.: MNHN exam. aut. - (Colômbia). - Lacordaire, 1842:335 (Brachysphoenus). - Crotch, 1876: 509. - Gemminger \& Harold, 1876:3707. - Kuhnt, 1909:26; 1911:12. Deelder, 1942:60. - Blackwelder, 1945:458. Distr.: Colômbia.

dispilotus Lacordaire, 1842:334 (Brachysphoenus). - Tp.: MCZC exam. aut. (Colômbia). - Crotch, 1876:508. - Gemminger \& Harold, 1876:3708. Kuhnt, 1909:26; 1911:14. - Schenkling, 1919:81. - Deelder, 1942:61. Blackwelder, 1945:458. Distr.: Colômbia.

var. immaculatus Deelder, 1942:61. Tp.: RNHL - (Colômbia, Cañca). Distr.: Colômbia.

ab. inversus Mader, 1942c:555. Tp. ? - (Colômbia). Distr.: Colômbia.

ecuadorensis Crotch, 1876:509 (Brachysphoenus). Tp.: MCZC exam. aut. -

(Equador). - Gemminger \& Harold, 1876:3708. - Kuhnt, 1909:26; 1911:

14. - Blackwelder, 1945:458. Distr.: Equador.

ellipticus Kuhnt, 1910:255 (Brachysphaenus). Tp. ? - (Venezuela, Mérida); 1911:14. - Blackwelder, 1945:458. Distr.: Venezuela.

luscus Erichson, 1847:178 (Brachysphenus). Tp.: ZMHU exam. aut. - (Peru). Crotch, 1876:523. - Gemminger \& Harold, 1876:3709. - Kuhnt, 1909:26; 1911:16. - Mader, 1942d: 158; 1944:105. - Blackwelder, 1945:459. Distr.: Peru.

oblongosignatus Guérin-Méneville, 1844:311 (Brachysphoenus). Tp.: MNHN exam. aut. - (Colômbia). - Crotch, 1876:509. - Gemminger \& Harold, 1876:3710. - Kuhnt, 1909:26; 1911:17. - Blackwelder, 1945:459. Distr.: Colômbia. 


\section{Iphiclus (Iphiclus) Chevrolat, 1837}

Iphiclus Chevrolat, 1837:426 (Tsubgen.: Erotylus flavovittatus Duponchel, 1825: 59, seg. Alvarenga, 1965:85). - Lacordaire, 1842:337. - Chapuis, 1876:59. - Crotch, 1876:509. - Kuhnt, 1909:22, 26. - Mader, 1944:105. - Alvarenga, 1965:85.

abdominalis Olivier, 1792:433 (Erotylus). Tp. ? - [Localidade não citada]; 1807:474, pl 2, fig. 18. - Fabricius, 1792:38; 1801:5. - Herbst, 1799:377. - Schönherr, 1808:327. - Duponchel, 1825:44, pl. 1, fig. 19. - Sturm, 1843:304 (Saccomorphus). - Lacordaire, 1842:398 (Brachysphoenus). Crotch, 1876:511. - Gemminger \& Harold, 1876:3706. - Kuhnt, 1909:29; 1911:11. - Bruch, 1914:381. - Deelder, 1942:60. - Blackwelder, 1945:457. - Guérin, 1948:5. Distr.: Brasil, regiões sudeste e sul.

atrisignatus Alvarenga, 1976:513 Distr.: Brasil, Minas Gerais.

conspersus; Duponchel, 1825, nec Germar, 1824. - Duponchel, 1825:57, pl. 2, fig. 48 (Erotylus). Tp.: MNHN exam. aut. - (Brasil). - Lacordaire, 1842:342 (Brachysphoenus). -Crotch, 1876:500. - Gemminger \& Harold, 1876:3708. - Kuhnt, 1909:23; 1911:14. - Blackwelder, 1945:458. - Alvarenga, 1976:513 (nom.praeoc.).

basalis Crotch, 1876:410 (Brachysphoenus). Tp.: MCZC exam. aut. - (Amazonas). - Gemminger \& Harold, 1876:3707. - Kuhnt, 1909:27; 1911:12. Blackwelder, 1945:458. Distr.: Brasil, região amazônica.

chelonarius Lacordaire, 1842:343 (Brachysphoenus). Tp.: MCZC exam. aut. (Brasil). - Crotch, 1876:512. - Gemminger \& Harold, 1876:3707. - Kuhnt, 1909:27; 1911:13. - Blackwelder, 1945:458. Distr.: Brasil.

cingulatus Lacordaire, 1842:341 (Brachysphoenus). Tp.: MCZC exam. aut. (Brasil) - Crotch, 1876:512. - Gemminger \& Harold, 1876:3707. - Kunth, 1909:27; 1911:13. - Blackwelder, 1945:458. Distr.: Brasil, região sudeste. claropictus Kuhnt, 1910:260 (Brachysphaenus). Tp. ZMHU exam. aut. - (Paraguai, San Pedro); 1911:13. - Blackwelder, 1945:458. Distr.: Paraguai.

decemnotatus Duponchel, 1825:45, pl. 1, fig. 22 (Erotylus). Tp. ? - (Brasil). Lacordaire, 1842:340 (Brachysphoenus). - Crotch, 1876:512. - Gemminger \& Harold, 1876:3708. - Kuhnt, 1909:27; 1911:14. - Schenkling, 1919:81. - Blackwelder, 1945:458. - Guérin, 1948:6. Distr.: Brasil, regiões sudeste e sul.

var. mediofasciatus Guérin, 1956:50. Tp.: CDSP exam. aut. - (Brasil, Paraná, Ponta Grossa). Distr.: Brasil, região sul.

var. rufonotatus Crotch, 1876:512. - Kunth, 1909:27; 1911:14. - Blackwelder, 1945:458.

flavovittatoides Mader, 1943:111 (Brachysphoenus). Tp. ? - (Brasil, Minas Gerais, Teófilo Otoni). Distr.: Brasil, região sudeste. 
flavovittatus Duponchel, 1825:59, pl. 2, fig. 55 (Erotylus). Tp. ? - (Brasil). Lacordaire, 1842:339 (Brachysphoenus). - Crotch, 1876:510. - Gemminger \& Harold, 1876:3709. - Kuhnt, 1909:26, pl. 2, fig. 4; 1911:15. Bruch, 1914:380. - Mader, 1942c:556. - Deelder, 1942:62. - Blackwelder, 1945:458. - Guérin, 1948:6. Distr.: Brasil, regiões sudeste e sul. Argentina.

flexuosus Lacordaire, 1842:400 (Brachysphoenus). Tp. ? - (Brasil, Goiás). Crotch, 1876:512. - Gemminger \& Harold, 1876:3709. - Kuhnt, 1909:30; 1911:15. - Deelder, 1942:62. - Blackwelder, 1945:458. Distr.: Brasil, regiões sudeste, sul e central.

guttatus Duponchel, 1825:52, pl. 2. fig. 37 (Erotylus). Tp. ? - (Brasil). Lacordaire, 1842:352 (Brachysphoenus). - Crotch, 1876:511. - Gemminger \& Harold, 1876:3709. - Kuhnt, 1909:27; 1911:15. - Deelder, 1942:62. - Blackwelder, 1945:458. Distr.: Brasil, região sudeste.

guttiger Kuhnt, 1910:261 (Brachysphoenus). Tp.: ZMHU exam. aut. - (Peru); 1911:15. - Mader, 1942d: 159; 1944:105. - Blackwelder, 1945:458. Distr.: Peru.

intersectus Duponchel, 1825:49, pl. 2, fig. 29 (Erotylus). Tp.: MNHN exam. aut. - (Brasil). - Lacordaire, 1842:131 (Ischyrus ?). - Crotch, 1876:512 (Brachysphoenus). - Gemminger \& Harold, 1876:3709. - Kuhnt, 1909:27; 1911:16. - Schenkling, 1919:81. - Deelder, 1942:62. - Blackwelder, 1945:459. - Guérin, 1948:6. Distr.: Brasil, regiões sudeste e sul.

lineellus Duponchel, 1825:58, pl. 2, fig. 51 (Erotylus). Tp.: MNHN exam. aut. - (Brasil). - Lacordaire, 1842:354 (Brachysphoenus). - Crotch, 1876:511.

- Gemminger \& Harold, 1876:3709. - Kuhnt, 1909:27; 1911:16. - Blackwelder, 1945:459. Distr.: Brasil, região sudeste.

amabilis Guérin-Méneville, 1941b:154 (Brachymerus). - Crotch, 1876:511 (syn.).

maculatus Voet, 1778:44, pl. 33, fig. 7 (Pseudochrysomela). Tp. ? - [Localidade não citada]. - Alvarenga, 1977:105 (Iphiclus). Distr.: Venezuela. Suriname. Guiana Francesa. Brasil, região amazônica.

sedecimguttatus Olivier, 1792:436 (Erotylus). Tp. ? - (Suriname); 1807:477, pl. 2, fig. 23. - Duponchel, 1825:53, pl. 2, fig. 39. - Lacordaire, 1842:348 (Brachysphoenus). - Crotch, 1876:511. - Gemminger \& Harold, 1876:3711. - Kuhnt, 1909:26; 1911:19. - Deelder, 1942:63. Blackwelder, 1945:460. - Alvarenga, 1977:105 (syn.).

ocellatus Kuhnt, 1910:262, fig. 19 (Brachysphaenus). - Tp.: ZMHU exam. aut. - (Brasil, Bahia); 1911:17. - Blackwelder, 1945:459. - Guérin, 1948:6. Distr.: Brasil, regiões sudeste e sul.

octodecimguttatus Lacordaire, 1842:351 (Brachysphoenus). Tp. ? - (Rio de Janeiro). - Crotch, 1876:511. - Gemminger \& Harold, 1876:3710. - Kuhnt, 1909:26; 1911:17. - Blackwelder, 1945:459. - Guérin, 1948:6. Distr.: Brasil, região sudeste. 
ab. atricollis Mader, 1942c:556. Tp. ? - (Brasil). Distr.: Brasil. pardalinus Lacordaire, 1842:355 (Brachysphoenus). Tp. ? - (Rio de Janeiro). Crotch, 1876:511. - Gemminger \& Harold, 1876:3710. - Kuhnt, 1909:27; 1911:18. - Blackwelder, 1945:459. Distr.: Brasil, região sudeste.

pictus Duponchel, 1825:48, pl. 1, fig. 28 (Erotylus). Tp.: MNHN exam. aut. (Brasil). - Lacordaire, 1842:341 (Brachysphoenus). - Crotch, 1876:512. Gemminger \& Harold, 1876:3710. - Kuhnt, 1909:27; 1911:18. - Blackwelder, 1945:459. Distr.: Brasil, região sudeste.

quadristictus Guérin, 1956:51, fig. 9 (Brachysphaenus). Tp.: FIOC exam. aut. (Brasil, Est. Rio de Janeiro, Itatiaia). Distr.: Brasil, região sudeste.

quinquevingitipunctatus Mader, 1943:112 (Brachysphoenus). Tp.: MGFM exam. aut. - (Brasil, Est. S. Paulo, Alto da Serra). Distr.: Brasil, região sudeste.

rubidus Duponchel, 1825:59, pl. 2, fig. 53 (Erotylus). Tp. ? - (Brasil). Lacordaire, 1842:338 (Brachysphoenus). - Crotch, 1876:510. - Gemminger \& Harold, 1876:3711. - Kuhnt, 1909:26; 1911:19. - Deelder, 1942:63. - Blackwelder, 1945:459. - Guérin, 1948:6. Distr.: Brasil, região sudeste.

scutellaris Gorham, 1888:98 (Brachysphenus). Tp.: NHML exam. aut. - (Guatemala, Cerro Zunil). - Kuhnt, 1909:26; 1911:19. - Blackwelder, 1945:460. Distr.: Guatemala. Nicarágua. Costa Rica.

sedecimmaculatus Buquet, 1840:173 (Iphiclus). Tp.: MNHN exam. aut. - (Colômbia). - Guérin-Méneville, 1841a:118 (Scaphidomorphus). - Lacordaire, 1842:348 (Brachysphoenus). - Crotch, 1876:510. - Gemminger \& Harold, 1876:3711. - Gorham, 1888:97, pl. 5, fig. 13. - Kuhnt, 1909:27; 1911:19. - Deelder, 1942:63. - Mader, 1942d:159; 1944:105. - Blackwelder, 1945: 460. Distr.: Panamá. Colômbia. Peru. Brasil. Paraguai.

var. concentratus Crotch, 1876:510. Tp. ? - (Colômbia). - Kuhnt, 1909:27; 1911:19. - Mader, 1942d:159; 1944:105. - Blackwelder, 1945:460. Distr.: Colômbia.

var. discedens Kuhnt, 1910:261. Tp.: ZMHU exam. aut. - [Localidade não citada]; 1911:19. - Mader, 1942d:159; 1944:105. - Blackwelder, 1945: 460 .

sedecimpustulatus Lacordaire, 1842:349 (Brachysphoenus). Tp.: MNHN exam. aut. - (Caiena). - Crotch, 1876:510. - Gemminger \& Harold, 1876:3711. - Kuhnt, 1909:26; 1911:19. - Deelder, 1942:63. - Blackwelder, 1945:460. - Guérin, 1948:9. Distr.: Brasil, região amazônica.

var. sanguinans Guérin, 1948:7, fig. 1. Tp.: MNRJ exam. aut. - (Brasil, Pará, Santarém). Distr.: Brasil, região amazônica.

sexpunctatus Duponchel, 1825:163, pl. 7, fig. 78 (Erotylus). Tp. ? - [Localidade não citada]. - Sturm, 1826:139. - Lacordaire, 1842:337 (Brachysphoenus). - Sturm, 1843:304. - Crotch, 1876:509. - Gemminger \& Harold, 1876: 3711. - Kuhnt, 1909:26; 1911:19. - Deelder, 1942:63. - Blackwelder, 
1945:460. - Guérin, 1948:9. Distr.: Brasil, região sudeste.

striolatus Guérin, 1956:51. fig. 8 (Brachysphaenus). Tp.: CDSP exam. aut. (Bolívia, Yungas del Palmar). Distr.: Bolívia.

tenuecinctus Lacordaire, 1842:340 (Brachysphoenus). Tp. ? - (Brasil). - Crotch,

1876:510. - Gemminger \& Harold, 1876:3712. - Kuhnt, 1909:26; 1911:20.

- Blackwelder, 1945:460. Distr.: Brasil, região sudeste.

titschacki Mader, 1942d:158, 184 (Brachysphoenus). Tp. ? - (Peru, Siva);

1944: 105. Distr.: Peru.

varians Lacordaire, 1842:344 (Brachysphoenus). Tp. ? - (Colômbia). - Crotch,

1876:509. - Gemminger \& Harold, 1876:3712. - Kuhnt, 1909:26; 1911:20.

- Schenkling, 1919:81. - Deelder, 1942:63. - Blackwelder, 1945:460.

Distr.: Colômbia. Brasil, região amazônica.

m-nigrum Lacordaire, 1842:346. Tp.: MNHN exam. aut. (Colômbia). Crotch, 1876:509 (syn.).

ab. atricollis Mader, 1942c:555. Tp. ? - (Colômbia). Distr.: Colômbia. Venezuela.

ventralis Lacordaire, 1842:399 (Brachysphoenus). Tp. ? - (Rio de Janeiro). Crotch, 1876:512. - Gemminger \& Harold, 1876:3712. - Kuhnt, 1909:29; 1911:20. - Blackwelder, 1945:460. - Guérin, 1948:6. Distr.: Brasil, região sudeste.

vigintipunctatus Olivier, 1792:436 (Erotylus). Tp. ? - (Suriname); 1807:477, pl. 2, fig. 24. - Crotch, 1876:511 (Brachysphoenus). - Gemminger \& Harold, 1876:3712. - Kuhnt, 1909:26; 1911:20. - Deelder, 1942:63. - Blackwelder, 1945:460. Distr.: Suriname. Brasil, região amazônica.

vigintiguttatus Germar, 1824:612 (Erotylus). - Lacordaire, 1842:350 (Brachysphoenus). - Crotch, 1876:511 (syn.).

vigintiguttatus Duponchel, 1825:53, pl. 2, fig. 40 (Erotylus). - Lacordaire, 1842:350 (Brachysphoenus). - Crotch, 1876:511 (syn.).

var. castaneus Guérin, 1956:52. Tp.: FIOC exam. aut. - (Brasil, Est. Rio de Janeiro, Itatiaia). Distr.: Brasil, região sudeste.

\section{Iphiclus (Aegithomorphus) Lacordaire, 1842}

Aegithomorphus Lacordaire, 1842:374 (Tsubgen.: Morphoides biplagiatus Guérin-Méneville, 1841a:119, seg. Alvarenga, 1965:81). - Chapuis, 1876:60. Crotch, 1876:513. - Kuhnt, 1909:22, 27. - Alvarenga, 1965:81.

biplagiatus Guérin-Méneville, 1841 a: 119 (Morphoides). Tp. ? - (Brasil). - Lacordaire, 1842:374 (Brachysphoenus). - Crotch, 1876:513. - Gemminger \& Harold, 1876:3707. - Kuhnt, 1909:27; 1911:12. - Blackwelder, 1945:458. Distr.: Brasil, região sudeste.

dorsomaculatus Lacordaire, 1842:375 (Brachysphoenus). Tp. ? - (Brasil). 
Crotch, 1876:513. - Gemminger \& Harold, 1876:3708. - Kuhnt, 1909:27; 1911:14. - Blackwelder, 1945:458. Distr.: Brasil, região sudeste.

\section{Iphiclus (Neoogaster) Alvarenga, 1965}

Neoogaster Alvarenga, 1965:87 (Tsubgen.: Erotylus marginatus Olivier, 1792: 437, monotipia). - Alvarenga, 1965:87.

Oogaster Lacordaire, 1842 nec Falderman, 1840. - Lacordaire, 1842:377. Chapuis, 1876:61. - Crotch, 1876:513. - Kuhnt, 1909:22, 27. - Alvarenga, 1965:88 (nom.praeoc.).

marginatus Olivier, 1792:437 (Erotylus). Tp. ? - (Caiena ?); 1807:482, pl. 1, fig.

8. - Gemminger \& Harold, 1876:3710 (Morphoides). - Kuhnt, 1911:16 (Brachysphaenus). - Deelder, 1942:62. - Blackwelder, 1945:459. Distr.: Guadeloupe. República Domonicana. Guiana Francesa ?

guadeloupensis Fabricius, 1792:16 (Galleruca). Tp.: ZMCD - (Guadeloupe); 1801:10 (Aegithus). - Duponchel, 1825:162, pl. 7, fig. 76 (Erotylus). - Lacordaire, 1842:377 (Brachysphoenus). - Gemminger \& Harold, 1876:3710 (syn.).

suturalis Lacordaire, 1842:378 (Brachysphoenus). - Crotch, 1876:513 (syn.).

\section{Iphiclus (Saccomorphus) Chevrolat, 1837}

Saccomorphus Chevrolat, 1837:426 (Tsubgen. Erotylus limbatus Olivier, 1792: 437, seg. Alvarenga, 1965:89). - Crotch, 1876:513. - Kuhnt, 1909:22, 27. Mader, 1944:106. - Alvarenga, 1965:89; 1977:103.

Charidemus Gistel, 1848:123 (Tgen. Chrysomela bimaculata Fabricius, 1775, monotipia). - Alvarenga, 1970:45 (syn.).

Morphoides Hope, 1841:111 (Tgen. Erotylus limbatus Olivier, 1792:437, desig. orig.). - Lacordaire, 1842:356. - Chapuis, 1876:60. - Crotch, 1876:515. Kuhnt, 1909:22, 28. - Mader, 1944:108. - Alvarenga, 1965:86 (syn.).

adamsi Lacordaire, 1842:366 (Brachysphoenus). Tp. ? - (Colômbia). - Crotch, 1876:515. - Gemminger \& Harold, 1876:3707. - Gorham, 1888:98. Kuhnt, 1909:28; 1911:11. - Mader, 1935:295; 1942d:160; 1944:108. Blackwelder, 1945:457. Distr.: Panamá. Colômbia. Peru. Brasil, região amazônica.

bilineatus Duponchel, 1825:166, pl. 7, fig. 85 (Erotylus). Tp. ? - (Brasil). Lacordaire, 1842:365 (Brachysphoenus). - Crotch, 1876:515. - Gemminger \& Harold, 1876:3707. - Dohrn, 1878:451. - Berg, 1881:110. Kolbe, 1907:79. - Kuhnt, 1909:28; 1911:12. - Bruch, 1914:380. - Mader, 1935:295. - Deelder, 1942:60. - Blackwelder, 1945:458. - Delkeskamp, 1957:96. Distr.: Brasil, região sul. Uruguai. Argentina. Bolívia. 
bimaculatus Fabricius, 1775:820 (Chrysomela). Tp. ? - (América). - Germar, 1824:612 (Erotylus). - Duponchel, 1825:41, pl. 1, fig. 13. - Gistel, 1848: 123 (Charidemus). - Lacordaire, 1842:358 (Brachysphoenus). - Crotch, 1876:513. - Gemminger \& Harold, 1876:3707. - Kuhnt, 1909:28; 1911:12. - Mader, 1935:294. - Deelder, 1942:60. - Blackwelder, 1945:458. Guérin, 1948:9. Distr.: Brasil, região sudeste.

bisigillatus Lacordaire, 1842:365 (Brachysphoenus). Tp. ? - (Colômbia). - Crotch, 1876:515. - Gemminger \& Harold, 1876:3707. - Kuhnt, 1909:28; 1911:12. - Mader, 1935:272. - Deelder, 1942:60. - Blackwelder, 1945:458. Distr.: Colômbia.

collaris Deelder, 1942:60 (Brachysphaenus). Tp.: RNHL - (Brasil ?). Distr.: Brasil?

erotyloides Crotch, 1876:515 (Brachysphoenus). Tp.: MCZC exam. aut. - (Amazonas). - Gemminger \& Harold, 1876:3708. - Kuhnt, 1909:28; 1911:15. Mader, 1935:272; 1942d:159; 1944:106. - Blackwelder, 1945:458. Distr.: Brasil, região amazônica. Peru. Bolívia.

erythrocephalus Olivier, 1807:475, pl. 2, fig. 19 (Erotylus). Tp. ? - (América meridional). - Duponchel, 1825:49, pl. 2, fig. 30. - Lacordaire, 1842:405 (Brachysphoenus). - Gemminger \& Harold, 1876:3708. - Deelder, 1942: 61. - Mader, 1942c:557. - Blackwelder, 1945:458. Distr.: Colômbia.

glyptoderus Lacordaire, 1842:361 (Brachysphoenus). Tp. ? - (Colômbia). Crotch, 1876:514. - Gemminger \& Harold, 1876:3709. - Kuhnt, 1909:28; 1911:15. - Mader, 1935:294; 1942d:160; 1944:108. - Blackwelder, 1945: 458. Distr.: Colômbia. Equador. Peru.

goianus Guérin, 1948:9, fig. 2 (Brachysphaenus). Tp.: MNRJ exam. aut. -(Brasil, Goiás, Goiatuba). Distr.: Brasil, região central.

haematocephalus Lacordaire, 1842:361 (Brachysphoenus). Tp.: MCZC exam. aut. - (Colômbia). - Crotch, 1876:514. - Gemminger \& Harold, 1876:3709. - Gorham, 1888:98; 1891:56. - Kuhnt, 1909:28; 1911:16. - Bruch, 1914:380. - Mader, 1935:293. - Blackwelder, 1945:459. Distr.: Panamá. Colômbia. Venezuela. Argentina.

humeralis Guérin, 1956:49, fig. 7 (Brachysphaenus). Tp.: CDSP exam. aut. (Brasil, Paraná, Monte Alegre). Distr.: Brasil, região sul.

intercedens Schenkling, 1919:82 (Brachysphaenus). Tp.: DEME exam. aut. (Peru, Ocobamba, Chanchamayo). - Mader, 1935:272; 1942d: 159; 1944: 106. - Blackwelder, 1945:459. Distr.: Peru. Bolívia.

interruptus Kuhnt, 1908b:632 (Brachysphaenus). Tp.: ZMHU exam. aut. (Bolívia); 1909:28; 1911:15. - Mader, 1935:272; 1942d:159; 1944:106. Deelder, 1942:61. - Blackwelder, 1945:458. - Delkeskamp, 1957:96. Distr.: Equador. Peru. Bolívia.

klugi Lacordaire, 1842:362 (Brachysphoenus). Tp.: MNHN exam. aut. - (Brasil meridional). - Crotch, 1876:514. - Gemminger \& Harold, 1876:3809. - 
Dohrn, 1878:451. - Kuhnt, 1909:28; 1911:16. - Bruch, 1914:380. - Mader, 1935:295. - Deelder, 1942:62. - Blackwelder, 1945:459. - Guérin, 1952: 180, fïg. 1. Distr.: Brasil, região sul. Argentina. Chile.

krafti Mader, 1935:271, 295 (Brachysphoenus). Tp.: MGFM exam. aut. - (Bolívia, Coroico). - Blackwelder, 1945:459. Distr.: Bolívia.

kuhnti Mader, 1942c:559 (Brachysphaenus). Tp.: MGFM exam. aut. - (Brasil, Amazonas, São Paulo de Olivença). Distr.: Brasil, região amazônica.

limbatus Olivier, 1792:437 (Erotylus). Tp. ? - (Caiena); 1807:477, pl. 2, fig. 25. - Fabricius, 1792:39; 1801:7. - Herbst, 1799:379. - Schönherr, 1808:328. - Duponchel, 1825:165, pl. 7, fig. 84. - Lacordaire, 1842:364 (Brachysphoenus). - Crotch, 1876:514. - Gemminger \& Harold, 1876:3709. Kuhnt, 1909:28; 1911:16. - Mader, 1935:295. - Blackwelder, 1945:459. Distr.: Guiana Francesa. Brasil.

maculicollis Kuhnt, 1910:254 (Brachysphaenus). Tp.: ZMHU - (Brasil); 1911:16. - Mader, 1944:109. - Blackwelder, 1945:459. Distr.: Brasil.

mundus Schenkling, 1919:82 (Brachysphaenus). Tp.: DEME exam. aut. - (Bolívia). - Mader, 1935:272. - Blackwelder, 1945:459. Distr.: Bolívia.

mutabilis Gorham, 1889:618 (Brachysphenus). Tp.; NHML exam. aut. - (Peru, Chanchamayo). - Kuhnt, 1909:27; 1911:17. - Mader, 1935:272; 1942d: 159; 1944:106. - Blackwelder, 1945:459. Distr.: Peru.

nebulosus Guérin-Méneville, 184 lb:155 (Ischyrus). Tp.: MNHN exam. aut. (Brasil). - Lacordaire, 1842:357 (Brachysphoenus). - Crotch, 1876:513. Gemminger \& Harold, 1876:3710. - Kuhnt, 1909:28; 1911:17. - Bruch, 1914:380. - Schenkling, 1919:81. - Mader, 1935:293; 1942d:159; 1944: 106. - Deelder, 1942:62. - Blackwelder, 1945:459. Distr.: Brasil, região central. Peru. Bolívia. Argentina.

var. nigricollis Mader, 1935:293. Tp. ? - (Peru); 1942d:159; 1944:107. Blackwelder, 1945:459. Distr.: Peru.

var. nigripes Mader, 1935:293. Tp. ? - [Localidade não citada]; 1944:107. Blackwelder, 1945:459. Distr.: ?.

var. ruber Guérin, 1956:49. Tp.: CDSP exam. aut. - (Brasil, Goiás, Itumbiara). Distr.: Brasil, região central.

var. unicoloripennis Mader, 1942d:160. Tp. ? - (Brasil, Mato Grosso, Corumbá); 1944:107. Distr.: Brasil, região central.

nitidus Mader, 1942c:556 (Brachysphoenus). Tp.: SMTD exam. aut. - (Bolívia). Distr.: Bolívia.

paraguayanus Mader, 1943:112 (Brachysphoenus). Tp. ? - (Paraguai). Distr.: Paraguai.

placitus Kirsch, 1876:108 (Erotylus). Tp.: SMTD exam. aut. - (Peru, Pozuzo). Gemminger \& Harold, 1876:3715. - Kuhnt, 1908a:93; 1909:36; 1911:25.

- Mader, 1942d:164; 1944:116. - Blackwelder, 1945:461. - Alvarenga, 1977: 104 (Iphiclus). Distr.: Peru. 
procerus Erichson, 1847:178 (Brachysphenus). Tp.: ZMHU exam. aut. - (Peru). - Crotch, 1876:523. - Gemminger \& Harold, 1876:3711. - Dohrn, 1883: 105. - Kuhnt, 1909:28; 1911:18. - Mader, 1942d:160; 1944:107. - Blackwelder, 1945:459. Distr.: Peru.

quadrisignatus Duponchel, 1825:166, pl. 7, fig. 86 (Erotylus). Tp. ? - (Brasil). Lacordaire, 1842:363 (Brachysphoenus). - Crotch, 1876:514. - Gemminger \& Harold, 1876:3711. - Kunth, 1908b:633; 1909:28; 1911:18. Bruch, 1914:380. - Schenkling, 1919:82. - Mader, 1935:294; 1942d:160; 1944: 107. - Deelder, 1942:62. - Blackwelder, 1945:459. - Guérin, 1948: 10. Distr.: Brasil, região sul. Peru. Bolívia. Paraguai. Argentina.

ruficeps Guérin-Méneville, 1841a:118 (Morphoides). Tp.: MNHN exam. aut. (Bolívia); 1855:611 (Brachysphaenus). - Lacordaire, 1842:359. - Crotch, 1876:514. - Gemminger \& Harold, 1876:3711. - Kuhnt, 1909:28; 1911:19. - Bruch, 1914:380. - Schenkling, 1919:81. - Mader, 1935:294; 1942d:160; 1944:107. - Deelder, 1942:63. - Blackwelder, 1945:459. - Delkeskamp, 1957:96. Distr.: Da Colômbia até a Argentina.

ab. immaculipennis Mader, 1935:294; 1942d:160; 1944:107. - Blackwelder, 1945:459. Distr.: Peru. Brasil.

terrestris Gistel, 1857:74 (Saccomorphus). Tp. ? - (Brasil). Distr.: Brasil. testaceipennis Mader, 1942c:558 (Brachysphoenus). Tp.: MGFM exam. aut. (Colômbia). Distr.: Colômbia.

\section{Iphiclus (Neomorphoides) Alvarenga, 1977}

Neomorphoides Alvarenga, 1977:103 (Tsubgen. Brachysphoenus (Morphoides) simplex Lacordaire, 1842:372, desig. orig.).

Morphoides; Crotch, 1876, nec Hope, 1841. - Alvarenga, 1977:103.

amazonus Crotch, 1876:516 (Brachysphoenus). Tp.: MCZC exam. aut. - (Amazonas). - Gemminger \& Harold, 1876:3707. - Kuhnt, 1909:28; 1911:12. Mader, 1944:110. - Blackwelder, 1945:457. Distr.: Brasil, região amazônica.

atriventris Mader, 1943:113 (Brachysphoenus). Tp.: MGFM exam. aut. - (Peru); 1944:110. Distr.: Peru.

bicolor Lacordaire, 1842:368 (Brachysphoenus). Tp. ? - (Brasil). - Crotch, 1876:516. - Gemminger \& Harold, 1876:3707. - Kuhnt, 1909:28; 1911:12. - Mader, 1944:110. - Blackwelder, 1945:458. - Guérin, 1948:9. Distr.: Brasil, região sudeste.

clavicornis Olivier, 1792:435 (Erotylus). Tp. ? - (Suriname); 1807:479, pl. 2, fig. 28. - Duponchel, 1825:42, pl. 1, fig. 14. - Sturm, 1843:304 (Saccomorphus). - Lacordaire, 1842:367 (Brachysphoenus). - Kuhnt, 1909:28; 1911:13. - Mader, 1942d:161; 1944:110. - Deelder, 1942:60. - Black- 
welder, 1945:458. - Guérin, 1948:9. Distr.: Da Colômbia até ao sul do Brasil.

lacordairei Crotch, 1876:515. - Gemminger \& Harold, 1876:3709 (syn.). columbiae Crotch, 1876:516 (Brachysphoenus). Tp.: MCZC exam. aut. - (Colômbia, Bogotá). - Gemminger \& Harold, 1876:3708. - Kuhnt, 1909:28; 1911:13. - Deelder, 1942:61. - Mader, 1944:110. - Blackwelder, 1945: 458. Distr.: Colômbia.

costaricensis Mader, 1943:113 (Brachysphoenus). Tp.: MGFM exam. aut. (Costa Rica); 1944:111. Distr.: Costa Rica.

disconigrum Mader, 1942:161, 185 (Brachysphoenus). Tp.: MGFM exam. aut. (Peru, Chanchamayo); 1944:109. Distr.: Peru.

dorsonotatus Lacordaire, 1842:370 (Brachysphoenus). Tp. ? - (Colômbia). Crotch, 1876:516. - Gemminger \& Harold, 1876:3708. - Kuhnt, 1909:28; 1911:14. - Deelder, 1942:61. - Mader, 1944:108. - Blackwelder, 1945: 458. Distr.: Colômbia. Brasil.

haematopterus Lacordaire, 1842:371 (Brachysphoenus). - Crotch, 1876:516 (syn.).

fulviventris Gorham, 1888:97 (Brachysphenus). Tp.: NHML - (Guatemala, San Gerónimo). - Kuhnt, 1909:28; 1911:15. - Mader, 1944:110. - Blackwelder, 1945:458. Distr.: Guatemala.

humeropictus Mader, 1943:113 (Brachysphoenus). Tp. ? - (Brasil); 1944:109. Distr.: Brasil.

lateripunctatus Crotch, 1876:517 (Brachysphoenus). Tp.: MCZC exam. aut. (Amazonas). - Gemminger \& Harold, 1876:3709. - Kuhnt, 1909:28; 1911:16. - Mader, 1944:109. - Blackwelder, 1945:459. - Guérin, 1948:10. Distr.: Equador. Peru. Brasil, região amazônica.

var. niger Guérin, 1956:49. Tp.: CDSP exam. aut. - (Peru, Huancayo, Satipo). Distr.: Peru.

melanopus Gorham, 1888:99 (Brachysphenus). Tp.: NHML - (Panamá, volcán de Chiriquí). - Kuhnt, 1909:28; 1911:17. - Mader, 1944:110. - Blackwelder, 1945:459. Distr.: Panamá.

neglectus Guérin, 1956:48, fig. 6 (Brachysphaenus). Tp.: CDSP exam. aut. (Brasil, Paraná, Ortigueira). Distr.: Brasil, região sul.

nigritarsis Mader, 1942d:161, 186 (Brachysphoenus). Tp.: MGFM exam. aut. (Peru, Puerto Inca, rio Pachitea); 1944:111. Distr.: Peru.

nigriventris Crotch, 1876:516 (Brachysphoenus). Tp.: MCZC exam. aut. - (Equador). - Gemminger \& Harold, 1876:3710. - Kuhnt, 1909:28; 1911:17. Mader, 1944:110. - Blackwelder, 1945:459. Distr.: Equador. Brasil, região amazônica.

nigropectus Mader, 1943:114 (Brachysphoenus). Tp. ? - (Brasil, Santa Catarina); 1944:111. Distr.: Brasil, região sul. 
posticenigrum Mader, 1942d:161, 185 (Brachysphoenus). Tp. ? -(Peru); 1944: 109. Distr.: Peru.

pyrrhocephalus Erichson, 1847:178 (Brachysphenus). Tp. ? - (Peru). - Crotch, 1876:522. - Gemminger \& Harold, 1876:3711. - Kuhnt, 1909:28; 1911:18.

- Mader, 1942d:160; 1944:108. - Blackwelder, 1945:459. Distr.: Peru.

rubripennis Lacordaire, 1842:372 (Brachysphoenus). Tp. ? - (Brasil). - Crotch, 1876:516. - Gemminger \& Harold, 1876:3711. - Kuhnt, 1909:28; 1911:19. - Deelder, 1942:63. - Mader, 1944:110. - Blackwelder, 1945:459. Guérin, 1948:10. Distr.: Brasil.

rufipennis Fabricius, 1801:8 (Erotylus). Tp.: ZMCD - (América meridional). Schönherr, 1808:328. - Duponchel, 1825:173. - Lacordaire, 1842:516. Gemminger \& Harold, 1876:3715. - Zimsen, 1964:115. - Chevrolat, 1843:79 (Morphoides). Distr.: Peru. Brasil.

immaculatus Lacordaire, 1842:369 (Brachysphoenus). Tp. ? - (Brasil). Crotch, 1876:516. - Gemminger \& Harold, 1876:3709. - Kunth, 1909:28; 1911:16. - Deelder, 1942:62. - Mader, 1942d:161; 1944:110. - Blackwelder, 1945:459. - Guérin, 1948:10. - Chevrolat, 1843:79 (syn.).

signaticollis Kuhnt, 1910:254 (Brachysphaenus). Tp.: ZMHU exam. aut. - (Brasil); 1911:19. - Mader, 1944:109. - Blackwelder, 1945:460. Distr.: Brasil. simplex Lacordaire, 1842:372 (Brachysphoenus). Tp. ? - (Brasil). - Crotch, 1876:517. - Gemminger \& Harold, 1876:3711. - Kuhnt, 1909:28; 1911:20. - Deelder, 1942:63. - Mader, 1942d:161; 1944:111. - Blackwelder, 1945: 460. - Guérin, 1948:10. - Delkeskamp, 1957:96. Distr.: Peru. Bolívia. Brasil.

spilotus Gorham, 1888:111, pl. 6 fig. 19 (Prepopharus). Tp.: NHML exam. aut. - (Panamá, Bugaba). - Kuhnt, 1909:44. - Arrow, 1909:196 (Brachysphoenus). - Kuhnt, 1911:20. - Mader, 1944:109. - Blackwelder, 1945:460. Distr.: Panamá.

tibialis Duponchel, 1825:55, pl. 2, fig. 44 (Erotylus). Tp. ? - (Brasil). - Lacordaire, 1842:373 (Brachysphoenus). - Crotch, 1876:517. - Gemminger \& Harold, 1876:3712. - Kuhnt, 1909:28; 1911:20. - Bruch, 1914:380. - Deelder, 1942:63. - Mader, 1944:111. - Balckwelder, 1945:460. - Guérin, 1948:10. Distr.: Brasil, regiões sudeste e sul. Argentina. 


\section{Iphiclus (Brachymerus) Chevrolat, 1837}

Brachymerus Chevrolat, 1837:427 (Tsubgen. Erotylus nitidulus Olivier, 1807:479, seg. Hope, 1841:110). Hope, 1841:110, 113. - Alvarenga, 1977:103.

Barytopus; Lacordaire, 1842, nec Duponchel \& Chevrolat, 1844. - Lacordaire, 1842:379. - Chapuis, 1876:61. - Crotch, 1876:517. - Kuhnt, 1909:22, 29. Mader, 1944:99. - Alvarenga, 1965:87 (= Neobarytopus).

Neobarytopus Alvarenga, 1965:87 (Tsubgen. Erotylus trincinctus Duponchel, 1824, desig. orig.). - Alvarenga, 1977:103 (= Brachymerus).

adustus Duponchel, 1825:56, pl. 2, fig.47 (Erotylus). Tp. ? - (Brasil). - Lacordaire, 1842:395 (Brachysphoenus). - Crotch, 1876:519. - Gemminger \& Harold, 1876:3707. - Kuhnt, 1909:29; 1911:11. - Blackwelder, 1945:457. Distr.: Brasil, região sudeste.

alboniger Guérin, 1956:52, fig. 10 (Brachysphaenus). Tp.: CDSP exam. aut. (Brasil, Rio Grande do Sul, Serro Azul). Distr.: Brasil, regiões central e sul.

var. picturatus Guérin, 1956:52. Tp.: CDSP exam. aut. - (Paraguai, Colônia Independência). Distr.: Paraguai.

amictus Erichson, 1847:178 (Brachysphenus). Tp.: ZMHU exam. aut. - (Peru). - Crotch, 1876:523. - Gemminger \& Harold, 1876:3707. - Kuhnt, 1909:29; 1911:12. - Mader, 1942d:154; 1944:100. - Blackwelder, 1945:457. Distr.: Peru.

andicola Kirsch, 1876:104 (Barytopus). Tp.: SMTD exam. aut. - (Peru, Pozuzo).

- Gemminger \& Harold, 1876:3707. - Kuhnt, 1909:22 (Brachysphaenus);

1911:12. - Mader, 1942d:154; 1944:99. - Blackwelder, 1945:458. Distr.: Peru.

ab. anteinterrupta Mader, 1942d:201. Tp. ? - (Peru); 1944:99. Distr.: Peru. assequens Mader, 1942b:79 (Brachysphoenus). Tp. ? - (Bolívia, Coroico). Distr.: Bolívia.

bajulus Lacordaire, 1842:413 (Brachysphoenus). Tp. ? - (Brasil). - Crotch, 1876:521. - Gemminger \& Harold, 1876:3707. - Kuhnt, 1909:31; 1911:12.

- Blackwelder, 1945:458. Distr.: Brasil.

batesi Gorham, 1889:617, pl. 61, fig. 7 (Brachysphenus). Tp.: NHML exam. aut. (Brasil, Amazonas). - Kuhnt, 1909:22; 1911:12. - Blackwelder, 1945:458. Distr.: Brasil, região amazônica.

bellulus Lacordaire, 1842:404 (Brachysphoenus). Tp. ? - (Caiena). - Crotch, 1876:521. - Gemminger \& Harold, 1876:3707. - Kuhnt, 1909:30; 1911:12.

- Deelder, 1942:60. - Blackwelder, 1945:458. Distr.: Colômbia. Guiana Francesa. Brasil, região amazônica.

bicinctus Olivier, 1807:472, pl. 2, fig. 15 (Erotylus). Tp.: MNHN exam. aut. (América meridional). - Duponchel, 1825:44, pl. 1, fig. 20. - Lacordaire, 
1842:381 (Brachysphoenus). - Crotch, 1876:503. - Gemminger \& Harold, 1876:3707. - Kuhnt, 1909:23; 1911:12. - Deelder, 1942:60. - Mader, 1942d:154; 1944:100. - Blackwelder, 1945:458. Distr.: Suriname. Peru. Brasil, região amazônica.

bistrifoliatus Gorham, 1889:616, pl. 61, fig. 5 (Brachysphenus). Tp.: NHML exam. aut. - (Peru, Chanchamayo). - Kuhnt, 1909:29; 1910:254; 1911:13. - Mader, 1942d:154; 1944:100. - Blackwelder, 1945:458. Distr.: Peru.

bizonatus Crotch, 1876:502 (Brachysphoenus). Tp.: MCZC exam. aut. - (Amazonas). - Gemminger \& Harold, 1876:3707. - Kuhnt, 1909:23; 1911:13. Blackwelder, 1945:458. Distr.: Brasil, região amazônica.

bremei Guérin-Méneville, 1841a:117 (Zonarius). Tp.: MCZC exam. aut. - (Bolívia). - Lacordaire, 1842:393 (Brachysphoenus). - Crotch, 1876:503. Gemminger \& Harold, 1876:3707. - Kuhnt, 1909:23; 1911:13. - Blackwelder, 1945:458. Distr.: Bolívia. Brasil, região central.

brongniarti Lacordaire, 1842:397 (Brachysphoenus). Tp.: MCZC exam. aut. (Colômbia). - Crotch, 1876:519. - Gemminger \& Harold, 1876:3707. Kuhnt, 1909:29; 1911:13. - Bruch, 1914:381. - Blackwelder, 1945:458. Delkeskamp, 1957:96. Distr.: Colômbia. Bolívia. Brasil. Argentina.

var. fasciatopunctatus Lacordaire, 1842:396. Tp. ? - (Bolívia, Santa Cruz de la Sierra). - Crotch, 1876:519. - Gemminger \& Harold, 1876:3707. Kuhnt, 1909:29; 1911:13. - Blackwelder, 1945:458. - Guérin, 1948:5. - Delkeskamp, 1957:96. Distr.: Bolívia. Brasil, região central.

brunneostriolatus Kuhnt, 1910:265, fig. 22 (Brachysphaenus). Tp.: ZMHU exam. aut. - (Brasil, Bahia); 1911:13. - Blackwelder, 1945:458. Distr.: Brasil, região sudeste.

cerasinus Lacordaire, 1842:400 (Brachysphoenus). Tp. ? - (Brasil). - Crotch, 1876:520. - Gemminger \& Harold, 1876:3707. - Kuhnt, 1909:29; 1911:13.

- Blackwelder, 1945:458. Distr.: Brasil.

conformis Lacordaire, 1842:353 (Brachysphoenus). Tp. ? - (Rio de Janeiro). Crotch, 1876:522. - Gemminger \& Harold, 1876:3708. - Kuhnt, 1909:31; 1911:13. - Blackwelder, 1945:458. - Guérin, 1948:6. Distr.: Brasil, região sudeste.

var. alienus Guérin, 1956:50. Tp.: CDSP exam. aut. - (Brasil, Amazonas, S.

Paulo de Olivença). Distr.: Bolívia. Brasil, região amazônica.

var. minor Guérin, 1956:50. Tp.: CARJ exam. aut. - (Brasil, Pará, Mocajuba, Mangabeira). Distr.: Brasil, região amazônica.

distinctus Duponchel, 1825:168 (Erotylus). Tp. ? - [Localidade não citada]. Lacordaire, 1824:394 (Brachysphoenus). - Crotch, 1876:519. - Gemminger \& Harold, 1876:3708. - Kuhnt, 1909:29; 1911:14. - Blackwelder, 1945:458. Distr.: Guiana Francesa.

divisus Guérin, 1956:53, fig. 11 (Brachysphaenus). Tp.: CDSP exam. aut. (Brasil, Amazonas, Benjamin Constant). Distr.: Brasil, região amazônica. 
dorsalis Olivier, 1792:436 (Erotylus). Tp.: MCZC exam. aut. - (Suriname); 1807:475, pl. 2, fig. 20. - Duponchel, 1825:59, pl. 2, fig. 54. - Lacordaire, 1842:388 (Brachysphoenus). - Crotch, 1876:518. - Gemminger \& Harold, 1876:3708. - Kuhnt, 1909:29; 1911:14. - Deeider, 1942:61. - Mader, 1942d:155, 177; 1944:101. - Blackwelder, 1945:458. - Guérin, 1948:6. Distr.: Peru. Bolívia. Suriname. Guiana Francesa. Brasil. Paraguai.

ab. quinquefasciatoides Mader, 1942d:155, 178. Tp. ? - [Localidade não citada]. Distr.: ?

eburneus Crotch, 1876:519 (Brachysphoenus). Tp.: MCZC exam. aut. - (Amazonas) - Gemminger \& Harold, 1876:3708. - Kuhnt, 1909:29; 1911:14. Blackwelder, 1945:458. Distr.: Brasil, região amazônica.

elegans Mader, 1942c:560 (Brachysphoenus). Tp. ? - (Brasil, S. Paulo, Alto da Serra). Distr.: Brasil, região sudeste.

epipleuralis Crotch, 1876:517 (Brachysphoenus). Tp.: MCZC exam. aut. (Amazonas). - Gemminger \& Harold, 1876:3708. - Kuhnt, 1909:29; 1911:15. - Deelder, 1942:61. - Mader, 1942d:154; 1944:100. - Blackwelder, 1945:458. Distr.: Peru. Brasil.

erichsoni Lacordaire, 1842:390 (Brachysphoenus). Tp.: MCZC exam. aut. (Colômbia). - Crotch, 1876:502. - Gemminger \& Harold, 1876:3708. Kuhnt, 1909:23; 1911:15. - Deelder, 1942:61. - Blackwelder, 1945:458. Distr.: Colômbia.

exoticus Voet, 1778:44, pl. 33, fig. 2 (Pseudochrysomela). Tp. ? - [Localidade não citada]. - Alvarenga, 1977:105 (Iphiclus). Distr.: ?

flavofasciatus Duponchel, 1825:43, pl. 1, fig. 18 (Erotylus). Tp. ? - (Brasil). Lacordaire, 1842:386 (Brachysphoenus). - Crotch, 1876:517. - Gemminger \& Harold, 1876:3708. - Kuhnt, 1909:30; 1911:15. - Bruch, 1914:381. - Schenkling, 1919:82. - Deelder, 1942:61. - Blackwelder, 1945:458. - Guérin, 1948:6. Distr.: Brasil, regiões sudeste e sul. Argentina.

var. apicalis Kuhnt, 1908b:633. Tp.: ZMHU exam. aut. - (Brasil); 1909:30; 1911:15. - Bruch, 1914:381. - Blackwelder, 1945:458. Distr.: Brasil, região Sul. Argentina.

flavosignatus Duponchel, 1825:57, pl. 2, fig. 49 (Erotylus). Tp. ? - (Brasil). Lacordaire, 1842:352 (Brachysphoenus). - Crotch, 1876:522. - Gemminger \& Harold, 1876:3709. - Kunth, 1909:30; 1911:15. - Deelder, 1942:62. - Blackwelder, 1945:458. - Guérin, 1948:6. Distr.: Brasil, região sudeste.

fragmentatus Gorham, 1888:99, pl. 5, fig. 16 (Brachysphenus). Tp.: NHML exam. aut. - (Guatemala, Capetillo). - Kuhnt, 1909:30; 1911:15. - Blackwelder, 1945:458. Distr.: Guatemala.

friedeli Mader, 1938:15, 17 (Brachysphoenus). Tp.: MGFM exam. aut. - (Colômbia, Cauca) [Localidade tipo foi trocada pela de Iphiclus laceratus]. - 
Blackwelder, 1945:458. Distr.: Colômbia.

geometra Lacordaire, 1842:381 (Brachysphoenus). Tp.: MNHN exam. aut.

(Caiena). - Crotch, 1876:503. - Gemminger \& Harold, 1876:3709. - Kuhnt,

1909:23; 1911:15. - Blackwelder, 1945:458. Distr.: Colômbia. Guiana

Francesa. Brasil, região amazônica.

hebraicus Lacordaire, 1842:403 (Brachysphoenus). Tp. ? - (Caiena). - Crotch,

1876:521. - Gemminger \& Harold, 1876:3709. - Kunth, 1909:30; 1911:16.

- Blackwelder, 1945:459. Distr.: Guiana Francesa.

hexastictus Crotch, 1876:520 (Brachysphoenus). Tp.: MCZC exam. aut. -(Ega) [atualmente Tefé]. - Gemminger \& Harold, 1876:3709. - Kuhnt, 1909:29;

1911:16. - Blackwelder, 1945:459. Distr.: Brasil, região amazônica.

var. discretus Crotch, 1876:520. Tp.: MCZC exam. aut. - (S. Paulo de Olivença). - Gemminger \& Harold, 1876:3709. - Kuhnt, 1909:29;

1911:16. - Blackwelder, 1945:459. Distr.: Brasil, região amazônica.

incas Gorham, 1889:617, pl. 61, fig. 4 (Brachysphenus). Tp.: NHML exam. aut.

- (Peru, Chanchamayo). - Kuhnt, 1909:23; 1911:16. - Deelder, 1942:62.

- Mader, 1944:100. - Blackwelder, 1945:459. Distr.: Peru.

iris Guérin, 1956:53, fig. 12 (Brachysphaenus). Tp.: CDSP exam. aut. - (Brasil, Amazonas, Maués). Distr.: Brasil, região amazônica.

jacinthoi Alvarenga, 1977:104. Distr.: Peru.

oculatus; Guérin, 1956, nec Duponchel 1825. Guérin, 1956:54, fig. 14 (Brachysphaenus). Tp.: CDSP exam. aut. -(Peru, Marcapata). - Alvarenga, 1977: 104 (nom.praeoc.).

laceratus Mader, 1938:14 (Brachysphoenus). Tp.: MGFM exam. aut. - (Brasil, Santa Catarina, Blumenau) [Localidade tipo foi trocada pela de Iphiclus friedeli]. - Blackwelder, 1945:459. - Guérin, 1946:369; 1948:6. Distr.: Brasil, região sul.

lugens Lacordaire, 1842:384 (Brachysphoenus). Tp. ? - (Colômbia). - Crotch, 1876:503. - Gemminger \& Harold, 1876:3709. - Kuhnt, 1909:23; 1911:16.

- Blackwelder, 1945:459. Distr.: Colômbia.

lunaris Guérin, 1956:54, fig. 13 (Brachysphaenus). Tp.: CDSP exam. aut. (Brasil, Pará, Obidos). Distr.: Brasil, região amazônica.

luteozonatus Crotch, 1876:518 (Brachysphoenus). Tp.: MCZC exam. aut. (Amazonas). - Gemminger \& Harold, 1876:3709. - Kuhnt, 1909:29; 1911:16. - Blackwelder, 1945:459. Distr.: Brasil, região amazônica.

miles Mader, 1942d:155, 180 (Brachysphoenus). Tp.: MGFM exam. aut. - (Peru, Marcapata); 1944:101. Distr.: Peru.

mirus Mader, 1942d: 155, 181 (Brachysphoenus). Tp.: MGFM exam. aut. - (Peru, Chanchamayo); 1944:101. Distr.: Peru.

musicalis Lacordaire, 1842:388 (Brachysphoenus). Tp.: MCZC exam. aut. (Colômbia). - Crotch, 1876:502. - Gemminger \& Harold, 1876:3710. - 
Kuhnt, 1909:22; 1911:17. - Blackwelder, 1945:459. Distr.: Colômbia. neophyta Lacordaire, 1842:415 (Brachysphoenus). Tp. ? - (Caiena). - Crotch, 1876:522. - Gemminger \& Harold, 1876:3710. - Kunth, 1909:30; 1911:17. - Blackwelder, 1945:459. Distr.: Guiana Francesa.

nigripennis Demay, 1838:24 (Erotylus). Tp.: MCZC exam. aut. - (Demerara). Lacordaire, 1842:412 (Brachysphoenus). - Crotch, 1876:522. - Gemminger \& Harold, 1876:3710. - Kuhnt, 1909:30; 1911:17. - Blackwelder, 1945:459. Distr.: Guiana.

nigropictus Lacordaire, 1842:387 (Brachysphoenus). Tp. ? -(Colômbia). - GuérinMéneville, 1855:611. - Crotch, 1873b:147; 1876:502. - Gemminger \& Harold, 1876:3710. - Gorham, 1888:99. - Kuhnt, 1909:22, 1911:17. Deelder, 1942:62. - Blackwelder, 1945:459. Distr.: Da América Central ao Brasil (região amazônica).

var. decoloratus Crotch, 1876:502. Tp.: MCZC exam. aut. - (Amazonas). Gemminger \& Harold, 1876:3710. - Kuhnt, 1909:22; 1911:17. Blackwelder, 1945:459. Distr.: Brasil, região amazônica.

ab. taeniatus Mader, 1942c:553. Tp. ? - (Colômbia). Distr.: Colômbia. nigrotrifasciatus Mader, 1942d:155, 178 (Brachysphoenus). Tp.: MGFM exam. aut. - (Peru, Oxapampa); 1944:101. Distr.: Peru.

nitidulus Olivier, 1807:479, pl. 2, fig. 29 (Erotylus). Tp. ? - (Guiana Francesa). - Duponchel, 1825:167. - Lacordaire, 1842:403 (Brachysphoenus).

Crotch, 1876:521. - Gemminger \& Harold, 1876:3710. - Kuhnt, 1909:30; 1911:17. - Deelder, 1942:62. - Blackwelder, 1945:459. Distr.: Guiana Francesa. Brasil, região amazônica.

obsoletesignatus Crotch, 1876:520 (Brachysphoenus). Tp.: MCZC exam. aut. (São Paulo). - Gemminger \& Harold, 1876:3710. - Kuhnt, 1909:29; 1911:17. - Deelder, 1942:62. - Blackwelder, 1945:459. Distr.: Brasil, região amazônica.

octoguttatus Olivier, 1807:485, pl. 3, fig. 39 (Erotylus). Tp. ? - (Caiena). Duponchel, 1825:175. - Lacordaire, 1842:412 (Brachysphoenus). - Crotch, 1876:522. - Gemminger \& Harold, 1876:3710. - Kuhnt, 1909:30; 1911:18. - Blackwelder, 1945:459. Distr.: Guiana Francesa.

oculatus Duponchel, 1825:161, pl. 7, fig. 73 (Erotylus). - Crotch, 1876:522 (syn.).

octopustulatus Guérin, 1956:56, fig. 18 (Brachysphaenus). Tp. : CDSP exam. aut. - (Brasil, Est. São Paulo, São Paulo, Jabaquara). Distr.: Brasil, região sudeste.

odyneroides Crotch, 1876:518 (Brachysphoenus). Tp.: MCZC exam. aut. -(Ega) [atualmente Tefé]. - Gemminger \& Harold, 1876:3710. - Kuhnt, 1909:29; 1911:18. - Mader, 1938:17. - Blackwelder, 1945:459. Distr.: Brasil, região amazônica.

ornatus Kuhnt, 1909:30 (Brachysphaenus). Tp.: ZMHU exam. aut. - (Brasil); 
1911:18. - Bruch, 1914:381. - Mader, 1942d:156; 1944:102. - Blackwelder, 1945:459. - Guérin, 1948:6. Distr.: Brasil, região amazônica. Argentina.

pantherinus Kuhnt, 1909:31 (Brachysphaenus). Tp.: ZMHU exam. aut. - (Brasil, Pará); 1911:18. - Blackwelder, 1945:459. Distr.: Brasil, região amazônica. pauper Guérin, 1956:56, fig. 17 (Brachysphaenus). Tp.: CDSP exam. aut. (Brasil, Est. São Paulo, São Paulo, Cantareira). Distr.: Brasil, regiões sudeste e sul.

peraffinis Crotch, 1876:502 (Brachysphoenus). Tp.: MCZC exam. aut. - (Amazonas). - Gemminger \& Harold, 1876:3710. - Kuhnt, 1909:23; 1911:18. Mader, 1938:16. - Blackwelder, 1945:459. Distr.: Equador. Brasil, região amazônica.

perplexus Mader, 1942d:155, 179 (Brachysphoenus). Tp. ? - (Peru, Chanchamayo); 1944:101. Distr.: Peru.

peruvianus Mader, 1942d:154, 177 (Brachysphoenus). Tp.: MGFM exam. aut. (Peru, Sátipo); 1944:99. Distr.: Peru.

planipennis Kuhnt, 1909:29 (Brachysphaenus). Tp.: ZMHU exam. aut. - (Suriname); 1911:18. - Blackwelder, 1945:459. Distr.: Suriname.

puncticollis Kirsch, 1876:104 (Brachysphenus). Tp.: SMTD exam. aut. - (Peru, Pozuzo). - Gemminger \& Harold, 1876:3711. - Kuhnt, 1909:30; 1911:18.

- Mader, 1942d:155, 156; 1944:102. - Blackwelder, 1945:459. Distr.: Peru.

quadrifasciatus Kirsch, 1865:98 (Brachysphenus). Tp.: SMTD exam. aut. (Colômbia, Bogotá). - Crotch, 1876:503. - Gemminger \& Harold, 1876: 3711. - Kuhnt, 1909:22; 1911:18. - Deelder, 1942:62. - Blackwelder, 1945:459. Distr.: Colômbia.

quinquefasciatus Lacordaire, 1842:396 (Brachysphoenus). Tp.: MCZC exam. aut. - (Colômbia). - Crotch, 1876:518. - Gemminger \& Harold, 1876:3711. Kuhnt, 1909:30; 1911:19. - Deelder, 1942:62. - Mader, 1942d:155, 178; 1944:101. - Blackwelder, 1945:459. Distr.: Equador. Colômbia. Brasil, região amazônica.

var. anisozonius Crotch, 1876:518. Tp.: MCZC exam. aut. - (Equador). Gemminger \& Harold, 1876:3711. - Kuhnt, 1909:30; 1911:19. Blackwelder, 1945:459. Distr.: Equador. Brasil, região amazônica.

var. basicinctus Crotch, 1876:518. Tp.: MCZC exam. aut. - (Amazonas). Gemminger \& Harold, 1876:3711. - Kuhnt, 1909:30; 1911:19. Blackwelder, 1945:459. Distr.: Brasil, região amazônica.

var. orthozonius Crotch, 1876:518. Tp.: MCZC exam. aut. - (Equador). Gemminger \& Harold, 1876:3711. - Kuhnt, 1909:30; 1911:19. Blackwelder, 1945:459. Distr.: Equador.

ramosus Olivier, 1807:480, pl. 2, fig. 30 (Erotylus). Tp.: MCZC exam. aut. (Caiena). - Duponchel, 1825:56, pl. 2, fig. 46. - Lacordaire, 1842:401 
(Brachysphoenus). - Crotch, 1876:520. - Gemminger \& Harold, 1876: 3711. - Kuhnt, 1909:29; 1911:19. - Deelder, 1942:62. - Blackwelder, 1945:459. Distr.: Colômbia. Equador. Suriname. Guiana Francesa. Brasil, região amazônica.

var. apicinctus Crotch, 1876:521. Tp.: MCZC exam. aut. - (Equador). Gemminger \& Harold, 1876:3711. - Kuhnt, 1909:29; 1911:19. Blackwelder, 1945:459. Distr.: Equador.

var. carmineus Crotch, 1876:521. Tp.: MCZC exam. aut. - (Equador). Gemminger \& Harold, 1876:3711. - Kuhnt, 1909:29; 1911:19. Blackwelder, 1945:459. - Guérin, 1948:6. Distr.: Equador. Brasil, região amazônica.

var. dilaceratus Lacordaire, 1842:402. Tp. ? - (Colômbia). - Crotch, 1876:

521. - Gemminger \& Harold, 1876:3711. - Kuhnt, 1909:29; 1911:19.

- Blackwelder, 1945:459. Distr.: Colômbia.

regularis Erichson, 1848:579 (Brachysphenus). Tp.: ZMHU exam. aut. - (Guiana). - Crotch, 1876:523. - Gemminger \& Harold, 1876:3711. - Kuhnt, 1909:29; 1911:19. - Mader, 1938:16. - Deelder, 1942:62. - Blackwelder, 1945:459. Distr.: Guiana. Suriname.

rhomboidalis Guérin, 1956:55, fig. 15 (Brachysphaenus). Tp.: CARJ - (Brasil, Pará, Mocajuba, Mangabeira). Distr.: Brasil, região amazônica.

var. integer Guérin, 1956:55. Tp.: CDSP exam. aut. - (Brasil, Pará, Obidos). Distr.: Brasil, região amazônica.

salamandra Erichson, 1847:178 (Brachysphenus). Tp.: ZMHU exam. aut. (Peru). - Crotch, 1876:3711. - Kunth, 1909:29; 1911:19. - Bruch, 1914: 381. - Mader, 1938:16; 1942d:155; 1944:100. - Blackwelder, 1945:460. Distr.: Equador. Peru. Bolívia. Brasil. Argentina.

spectabilis Lacordaire, 1842:392 (Brachysphoenus). Tp. ? - (Colômbia). - Crotch, 1876:519. - Gemminger \& Harold, 1876:3711. - Gorham, 1888:100. Kuhnt, 1909:29; 1911:20. - Mader, 1938:16. - Blackwelder, 1945:460. Distr.: Panamá. Colômbia.

stramineus Lacordaire, 1842:415 (Brachysphoenus). Tp. ? - (Colômbia). - Crotch, 1876:522. - Gemminger \& Harold, 1876:3711. - Kuhnt, 1909:30; 1911:20.

- Blackwelder, 1945:460. Distr.: Colômbia.

subsanguineus Crotch, 1876:521 (Brachysphoenus). Tp.: MCZC exam. aut. - (São Paulo). - Gemminger \& Harold, 1876:3711. - Kuhnt, 1909:30; 1911:20. Blackwelder, 1945:460. Distr.: Brasil, região amazônica.

var. disjunctus Crotch, 1876:521. Tp. ? - (Santarém). - Gemminger \& Harold, 1876:3711. - Kuhnt, 1909:30; 1911:20. - Blackwelder, 1945:460. Distr.: Brasil, região amazônica.

superbus Mader, 1942c:561 (Brachysphoenus). Tp.: MGFM exam. aut. - (Brasil, Amazonas, São Paulo de Olivença). Distr.: Brasil, região amazônica. tigrinatus Guérin, 1956:55, fig. 16 (Brachysphaenus). Tp.: CDSP exam. aut. - 
(Brasil, Amazonas, Borba). Distr.: Brasil, região amazônica.

tricinctus Duponchel, 1825:42, pl. 1, fig. 16 (Erotylus). Tp. ? - (Caiena). Lacordaire, 1842:385 (Brachysphoenus). - Kirsch, 1876:104. - Crotch, 1876:501. - Gemminger \& Harold, 1876:3712. - Kuhnt, 1909:23; 1911:20.

- Mader, 1938:16; 1942d:154; 1944:100. - Deelder, 1942:63. - Blackwelder, 1945:460. - Guérin, 1948:6. Distr.: Peru. Guiana Francesa. Brasil, região amazônica.

var. rectesignatus Crotch, 1876:501. Tp.: MCZC exam. aut. - (Amazonas). - Gemminger \& Harold, 1876:3712. - Kuhnt, 1909:23, pl. 2, fig. 16; 1911:20. - Mader, 1938:16; 1942d:154; 1944:100. - Blackwelder, 1945:460. Distr.: Brasil, região amazônica.

trifasciatus Olivier, 1807:473, pl. 2, fig. 16 (Erotylus). Tp. ? - (Brasil).

Duponchel, 1825:43, pl. 1, fig. 17. - Lacordaire, 1842:384 (Brachysphoenus). Distr.: Brasil, regiões sudeste e sul. Paraguai. Argentina.

fasciatus Olivier, 1792:433 (Erotylus). - Crotch, 1876:501 (Brachysphoenus).

- Gemminger \& Harold, 1876:3708. - Kuhnt, 1909:22; 1911:15. Bruch, 1914:381. - Schenkling, 1919:82. - Mader, 1938:16. - Deelder, 1942:61. - Blackwelder, 1945:458. - Guérin, 1948:6. - Olivier, 1807: 473 (nom.praeoc.).

tripartitus Lacordaire, 1842:386 (Brachysphoenus). Tp. ? - (Colômbia). - Crotch, 1876:519. - Gemminger \& Harold, 1876:3712. - Kuhnt, 1909:29; 1911:20.

- Blackwelder, 1945:460. Distr.: Colômbia.

ucayalensis bolivianus Mader, 1942:156 (Brachysphoenus). Tp.: NHMW (Bolívia, Coroico); 1944:102. Distr.: Bolívia.

ucayalensis ucayalensis Gorham, 1889:615, pl. 61, fig. 6 (Brachysphenus). Tp.: NHML exam. aut. - (Peru, rio Ucayale). - Kuhnt, 1909:26; 1911:20. Schenkling, 1919:81. - Mader, 1942d:155, 156; 1944:102. - Blackwelder, 1945:460. - Guérin, 1948:6. Distr.: Peru. Brasil, região amazônica.

venezuelae Crotch, 1876:519 (Brachysphoenus). Tp. ? - (Venezuela). - Gemminger \& Harold, 1876:3712. - Kuhnt, 1909:29; 1911:20. - Mader, 1938:16. - Blackwelder, 1945:460. Distr.: Venezuela.

westwoodi Guérin-Méneville, $1841 \mathrm{a}: 117$ (Zonarius). Tp.: MNHN exam. aut. (Bolívia). - Lacordaire, 1842:391 (Brachysphoenus). - Crotch, 1876:502. - Gemminger \& Harold, 1876:3712. - Kuhnt, 1909:23; 1911:20. - Mader, 1942d:154; 1944:99. - Blackwelder, 1945:460. - Guérin, 1948:6. Distr.: Peru. Bolívia. Brasil, região amazônica. 


\section{Typocephalus Chevrolat, 1837}

Typocephalus Chevrolat, 1837:427 (Tgen. Erotylus dimidiatus Olivier, 1792:435, seg. Hope, 1841:113). - Hope, 1841:113. - Crotch, 1876:523. - Alvarenga, 1965:90.

Cytorea Laporte, 1840:224. - Kuhnt, 1909:9, 31. - Deelder, 1942:51. - Alvarenga, 1965:83 (syn.).

cruciatus Lacordaire, 1842:407 (Brachysphoenus). Tp. ? - (Caiena). - Gemminger \& Harold, 1876:3706 (Cytorea). - Kuhnt, 1909:31; 1911:21. - Blackwelder, 1945:460. - Crotch, 1876:524 (Typocephalus). Distr.: Colômbia. Guiana Francesa.

dimidiatus Olivier, 1792:435 (Erotylus). Tp. ? - (Suriname); 1807:481, pl. 3, fig. 31. - Duponchel, 1825:176. - Lacordaire, 1842:406 (Brachysphoenus). Gemminger \& Harold, 1876:3706 (Cytorea). - Kuhnt, 1909:31; 1911:21.

- Blackwelder, 1945:460. - Crotch, 1876:523 (Typocephalus). Distr.:

Colômbia. Guiana. Suriname. Guiana Francesa. Brasil, região amazônica. bicolor Laporte, 1840:224 (Cytorea). - Lacordaire, 1842:406 (syn.).

interpunctatus Crotch, 1876:524. Tp.: MCZC exam. aut. - (Venezuela). - Gemminger \& Harold, 1876:3706 (Cytorea). - Kuhnt, 1909:31, pl. 2, fig. 8; 1911:21. - Blackwelder, 1945:460. Distr.: Venezuela.

tricolor Kuhnt, 1910:266. Tp.: ZMHU exam. aut. - (Oriba, Amazonas); 1911:21

(Cytorea). - Blackwelder, 1945:460. Distr.: Brasil, região amazônica.

vespoides Crotch, 1876:524. Tp.: MCZC exam. aut. - (Amazonas). - Gemminger \& Harold, 1876:3706 (Cytorea). - Kuhnt, 1909:31; 1911:21. - Blackwelder, 1945:460. Distr.: Brasil, região amazônica.

\section{Kakoia Alvarenga, 1971}

Kakoia Alvarenga, 1971c:35 (Tgen. Kakoia travassosi Alvarenga, 1971, monotipia).

travassosi Alvarenga, 1971:35. Tp.: CARJ - (Brasil, Est. São Paulo, Barueri). Distr.: Brasil, regiões sudeste e central.

Sphenoxus Lacordaire, 1842

Sphenoxus Lacordaire, 1842:376 (Tgen. Brachysphoenus germari Lacordaire, 1842:376, seg. Crotch, 1876:524). - Chapuis, 1876:60. - Crotch, 1876:524 (Sphenoxus). - Kuhnt, 1909:9, 31. - Deelder, 1942:51. - Alvarenga, 1965:89.

germari Lacordaire, 1842:376 (Brachysphoenus). Tp. ? - (Colômbia). - Gemminger \& Harold, 1876:3709 (Morphoides). - Crotch, 1876:524 (Sphenoxus). - Kuhnt, 1909:31; 1911:21. - Blackwelder, 1945:460. Distr.: Colômbia. 


\section{Eurycardius Lacordaire, 1842}

Eurycardius Lacordaire, 1842:32, 479 (Tgen. Eurycardius erythropterus Lacordaire, 1842:480, monotipia). - Chapuis, 1876:49, 65. - Crotch, 1876:524. Kuhnt, 1909:9, 32. - Deelder, 1942:51. - Alvarenga, 1965:84.

concolor Crotch, 1876:524. Tp.: MCZC exam. aut. - (Brasil, Minas Gerais). Gemminger \& Harold, 1876:3717. - Kuhnt, 1909:32; 1911:21. - Blackwelder, 1945:460. Distr.: Brasil, regiões sudeste e central.

erythropterus Lacordaire, 1842:480. Tp. ? - (Caiena). - Crotch, 1876:524. Gemminger \& Harold, 1876:3717. - Kuhnt, 1909:32; 1911:21. - Blackwelder, 1945:460. Distr.: Guiana Francesa. Brasil.

seabrai Alvarenga, 1971a:117. Tp.: UFPR - (Brasil, Espírito Santo, Barra de São Francisco, Corrego Itá). Distr.: Brasil, região sudeste.

\section{Dichomorpha Kuhnt, 1909}

Dichomorpha Kuhnt, 1909:9, 32 (Tgen. Dichomorpha fulva Kuhnt, 1909:33, monotipia). - Deelder, 1942:51. - Alvarenga, 1965:83.

fulva Kuhnt, 1909:33, pl. 2, figs 7, 7a. Tp. ? - (Colômbia); 1911:21. - Blackwelder, 1945:460. Distr.: Colômbia.

\section{Erotylus Fabricius, 1775}

Erotylus Fabricius, 1775:123 (Tgen. Coccinella gigantea Linnaeus, 1758:368, seg. Hope, 1841:110); 1787:91; 1792:35; 1798:101; 1801:3. - Olivier, 1792:429; 1807:465. - Duponchel, 1825:30. - Hope, 1841:110. - Lacordaire, 1842:32, 416. - Crotch, 1873b:148; 1876:525. - Chapuis, 1876:49, 62. - Gorham, 1888:101. - Kuhnt, 1908a:67; 1909:10, 33. - Curran, 1941:283. - Deelder, 1942:51. - Alvarenga, 1965:84.

Pseudochrysomela Voet, 1778:43. - Alvarenga, 1970:45 (syn.).

aegrotus Lacordaire, 1842:420. Tp. ? - (Brasil, Bahia). - Crotch, 1876:525. Gemminger \& Harold, 1876:3713. - Kuhnt, 1908a:72, 73; 1909:34; 1911:21. - Bruch, 1914:382. - Deelder, 1942:63. - Blackwelder, 1945:460.

- Guérin, 1948:13. Distr.: Brasil, região sudeste e sul. Argentina.

dryas Lacordaire, 1842:424. - Crotch, 1876:525 (syn.).

aequatoris Kirsch, 1883:211. Tp.: SMTD exam. aut. - (Equador, Baños-Pintuc). - Fleutiaux, 1886:224. - Kuhnt, 1908a:92; 1909:36; 1911:22. - Blackwelder, 1945:460. Distr.: Equador.

apiatoides Mader, 1942c:565. Tp. ? - (Brasil, Manaus). Distr.: Brasil, região amazônica. 
aulicus Lacordaire, 1842:438. Tp.: MNHN exam. aut. - (Caiena). - Crotch, 1876:536. - Gemminger \& Harold, 1876:3713. - Kuhnt, 1908a:100; 1909:38; 1911:22. - Blackwelder, 1945:460. Distr.: Guiana Francesa.

bellopictus Kuhnt, 1910:266, fig. 23. Tp.: ZMHU exam. aut. - (Argentina, Misiones); 1911:22. - Bruch, 1914:382. - Blackwelder, 1945:460. Distr.: Argentina.

bibalteatus Alvarenga, 1976:511. Distr.: Equador.

bifasciatus; Crotch, 1876, nec Olivier, 1792. - Crotch, 1876:532. Tp.: MCZC exam. aut. - (Equador). - Gemminger \& Harold, 1876:3713. - Kuhnt, 1908a:92; 1909:36; 1911:22. - Blackwelder, 1945:460. - Alvarenga, 1976:511 (nom.praeoc.).

boylei Alvarenga, 1971b:149. Tp.: UFPR - (Brasil, Pará, Óbidos). Distr.: Brasil, região amazônica.

buckleyi Crotch, 1876:527. Tp.: MCZC exam. aut. - (Equador). - Gemminger \& Harold, 1876:3713. - Kuhnt, 1908a:83, 84; 1909:35; 1911:22. - Deelder, 1942:63. - Blackwelder, 1945:460. Distr.: Equador.

var. catenatus Kuhnt, 1908a:83, 85. Tp. ? - [Localidade não citada]; 1911:22.

- Blackwelder, 1945:460. Distr.: Equador.

cassidoides Crotch, 1876:533. Tp.: MCZC exam. aut. - (Amazonas). - Gemminger \& Harold, 1876:3713. - Dohrn, 1880:293. - Kuhnt, 1908a:99; 1909:37, pl. 1, fig. 2; 1911:22. - Blackwelder, 1945:460. - Guérin, 1948:15. Distr.: Brasil, região amazônica.

chevrolati Lacordaire, 1842:421. Tp.: MCZC exam. aut. - (Brasil). - Crotch, 1876:525. - Gemminger \& Harold, 1876:3713. - Kuhnt, 1908a:71, 73; 1909:34; 1911:22. - Blackwelder, 1945:460. - Guérin, 1948:15. Distr.: Brasil, região sudeste.

cingulatus Crotch, 1876:530. Tp.: MCZC exam. aut. - (Amazonas). - Gemminger \& Harold, 1876:3713. - Kuhnt, 1908a:85; 1909:35; 1911:22. - Blackwelder, 1945:460. Distr.: Brasil, região amazônica.

clarosignatus Kuhnt, 1908a:72, 74. Tp.: ZMHU exam. aut. - (Brasil); 1909:34; 1911:22. - Deelder, 1942:63. - Blackwelder, 1945:460. Distr.: Brasil.

contractus Kuhnt, 1908a:86. Tp.: ZMHU exam. aut. - (Bolívia); 1909:35; 1911:22. - Blackwelder, 1945:460. - Delkeskamp, 1957:97. Distr.: Bolívia.

cornaliae Guérin-Méneville, 1855:611. Tp.: MNHN exam. aut. - [Localidade não citada]. - Crotch, 1876:536. - Gemminger \& Harold, 1876:3713. - Kuhnt, 1908a:93; 1909:36; 1911:22. - Blackwelder, 1945:460. Distr.: Equador.

crotchi Deelder, 1942:63, fig. 1c. Tp.: RNHL - (Peru, Chanchamayo). Distr.: Peru.

crucifer Kuhnt, 1908a:85. Tp.: ZMHU exam. aut. - (Brasil); 1909:35; 1911:22.

- Mader, 1942d:163; 1944:115. - Blackwelder, 1945:460. - Guérin, 
1948: 15. Distr.: Peru. Brasil, região amazônica.

decipiens Crotch, 1876:536. Tp.: MCZC exam. aut. - (Equador). - Gemminger

\& Harold, 1876:3713. - Kuhnt, 1908a:100; 1909:38; 1911:22. - Blackwelder, 1945:460. Distr.: Equador.

var. peruvianus Kuhnt, 1908a:100. Tp. ? - (Peru, Chanchamayo); 1909:38;

1911:22. - Mader, 1938:17. Distr.: Peru.

var. kuhnti Mader, 1938:17. Tp. ? - (Peru, Chanchamayo); 1944:117. Distr.: Peru.

delenitus Alvarenga, 1976:511. Distr.: Venezuela.

dilaceratus Kirsch, 1876:106. Tp.: SMTD exam. aut. - (Peru, Sarayacu). -

Gemminger \& Harold, 1876:3714. - Kuhnt, 1908a:89; 1909:36; 1911:22.

- Mader, 1942d:163; 1944:114. - Blackwelder, 1945:460. Distr.: Peru.

egregius Guérin, 1946b:365, fig. 1. Tp.: CDSP exam. aut. - (Brasil, Amazonas,

Maués). Distr.: Brasil, região amazônica.

elegans Kuhnt, 1908a:71, 73. Tp.: ZMHU exam. aut. - (Brasil); 1909:34; 1911:22. - Deelder, 1942:64. - Blackwelder, 1945:460. - Guérin, 1948:15.

Distr.: Brasil, região sudeste.

elegantulus Guérin, 1946b:366, fig. 2. Tp.: CDSP exam. aut. - (Brasil, Amazonas, Maués). Distr.: Brasil, região amazônica.

elongatulus Crotch, 1876:533. Tp.: MCZC exam. aut. - [Localidade não citada].

- Fleutiaux, 1886:224. - Kuhnt, 1908a:91; 1909:36; 1911:22. - Mader, 1942d:162; 1944:113. - Blackwelder, 1945:460. Distr.: Peru.

flavopunctatus Kuhnt, 1908a:78, 82; Tp.: ZMHU exam. aut. - (Peru, Marcapata); 1909:35; 1911:22. - Mader, 1942d:162; 1944:113. - Blackwelder, 1945: 460. Distr.: Peru.

flavotaeniatus Kuhnt, 1908a:75, 81. Tp. ? - (São Paulo ?); 1909:34; 1911:22. Blackwelder, 1945:460. Distr.: Brasil.

forsteri Delkeskamp, 1957:97, 101. Tp.: ZSBS exam. aut. - (Bolívia, Chapare, alto rio Chipiriri). Distr.: Bolívia.

fulvofasciatus Kuhnt, 1908a:88. Tp. ? - (Brasil, rio Juruá); 1909:36; 1911:22. Blackwelder, 1945:460. Distr.: Brasil.

giganteus Linnaeus, 1758:368 (Coccinella). Tp. ? - (Índia). - Sulzer, 1761, pl. 3, fig. 15a. - Houttuyn, 1766:74, fig. 3 (Chrysomela). - Degeer, 1775:349, pl. 16, fig. 8; 1781:416, pl. 16, fig. 8. - Fabricius, 1775:123 (Erotylus); 1781:157; 1787:91; 1792:35; 1801:3. - Sulzer, 1776, pl. 3, fig. 8. Roemer, 1789:43. - Gmelin, 1790:1726. - Olivier, 1792:432; 1807:468, pl. 1, fig. 6. - Herbst, 1799:360, pl. 136, fig. 9. - Latreille, 1804:57. Illiger, 1806:230. - Schönherr, 1808:325. - Duponchel, 1825:37, pl. 1, fig. 5. - Lacordaire, 1842:434. - Fauvel, 1860:326. - Crotch, 1876:529. Gemminger \& Harold, 1876:3714. - Dohrn, 1878:449. - Kuhnt, 1908a:79, 82; 1909:35; 1911:22. - Deelder, 1942:64. - Blackwelder, 1945:460. - 
Guérin, 1948:15. Distr.: Suriname. Guiana Francesa. Brasil, região amazônica.

paramariboensis Voet, 1778:44, pl. 33, fig.4 (Pseudochrysomela). - Alvarenga, 1977:105 (syn.).

glaber Kuhnt, 1908a:93. Tp. ? - (Equador, Baiza); 1909:36; 1911:23. - Blackwelder, 1945:460. Distr.: Equador.

guerini Demay, 1838:23. Tp.: MCZC exam. aut. - (Demerara). - Lacordaire, 1842:430. - Crotch, 1876:528. - Gemminger \& Harold, 1876:3714. Kuhnt, 1908a:89; 1909:36; 1911:23. - Blackwelder, 1945:460. - Delkeskamp, 1957:97. Distr.: Guiana. Bolívia.

hexagrammus Lacordaire, 1842:430. Tp.: MNHN exam. aut. - (Bolívia). - Blanchard, 1843, pl. 25, fig. 7. - Crotch, 1876:528. - Gemminger \& Harold, 1876:3714. - Kuhnt, 1908a:76, 81; 1909:34; 1911:23. - Deelder, 1942:64. - Blackwelder, 1945:460. - Delkeskamp, 1957:97. Distr.: Bolívia.

hieroglyphicus; Crotch, 1876, nec Duponchel 1825. - Crotch, 1876:532. Тр.: MCZC exam. aut. - (Venezuela). - Gemminger \& Harold, 1876:3714. Kuhnt, 1908a:91; 1909:36; 1911:23. - Blackwelder, 1945:460. - Alvarenga, 1976:511 (nom.praeoc.).

va1 columbiae Kuhnt, 1908a:91. Tp. ? - (Colômbia); 1909:36; 1911:23. Blackwelder, 1945:460. Distr.: Colômbia.

histrio Fabricius, 1787:91. Tp.: HCGU - (Caiena); 1792:36; 1801:4. - Gmelin, 1790:1727. - Olivier, 1792:432; 1807:468, pl. 2, fig. 12. - Herbst, 1799:376. - Schönherr, 1808:325. - Duponchel, 1825:36, pl. 1, fig. 3. Sturm, 1826:139; 1843:304. - Lacordaire, 1842:419. - Gistel, 1848:123. - Crotch, 1876:525. - Gemminger \& Harold, 1876:3714. - Kuhnt, 1908a: 69, 73; 1909:34; 1911:23. - Staig, 1931:104. - Deelder, 1942:64. Blackwelder, 1945:460. - Guérin, 1948:15. - Zimsen, 1964:114. Distr.: Guiana Francesa. Brasil.

var. minor Deelder, 1942:64. Tp.: RNHL - [Localidade não citada].

histrionicus Duponchel, 1825:37, pl. 1, fig. 4. Tp.: MNHN exam. aut. - (Brasil). - Lacordaire, 1842:422. - Crotch, 1876:525. - Gemminger \& Harold, 1876:3714. - Kuhnt, 1908a:71, 73; 1909:34; 1911:23. - Blackwelder, 1945:460. Distr.: Brasil.

imitans Kirsch, 1876:105. Tp.: SMTD exam. aut. - (Peru, Sarayacu). - Gemminger \& Harold, 1876:3714. - Kuhnt, 1908a:75, 81; 1909:34; 1911:23. Mader, 1942d:162; 1944:113. - Blackwelder, 1945:460. Distr.: Peru. Brasil, região amazônica.

incertus Lacordaire, 1842:435. Tp.: MCZC exam. aut. - (Caiena). - Erichson, 1848:578. - Crotch, 1876:529. - Gemminger \& Harold, 1876:3714. Dohrn, 1878:449. - Kuhnt, 1908a:79, 82; 1909:35; 1911:23. - Mader, 1942d:162; 1944:113. - Blackwelder, 1945:460. Distr.: Peru. Guiana Francesa. 
incomparabilis Perty, 1832:110, pl. 22, fig. 8. Tp. ? - (Brasil, rio Amazonas). Lacordaire 1842:467; 1876, pl. 132, fig. 4. - Crotch, 1876:528. - Gemminger \& Harold, 1876:3714. - Dohrn, 1878:451; 1880:291. - Kuhnt, 1908a: 87; 1909:36; 1911:23. - Mader, 1942d:163; 1944:114. - Blackwelder, 1945:460. Distr.: Peru. Brasil, região amazônica.

involutus Kuhnt, 1908a:91. Tp.: ZMHU exam. aut. - (Honduras, rio Manes); 1909:36; 1911:24. - Blackwelder, 1945:460. Distr.: Honduras.

isequeboensis Voet, 1778:44, pl. 33, fig. 5 (Pseudochrysomela). Tp. ? [Localidade não citada]. - Alvarenga, 1977:105 (Erotylina). comb.n. Distr.: Guiana. Suriname. Guiana Francesa. Brasil, região amazônica.

variegatus Fabricius, 1781:157 (Erotylus). Tp.: ZMCD - (Suriname). - 1787: 92; 1792:37; 1801:5. - Olivier, 1792:434; 1807:470, pl. 1, fig. 7. Herbst, 1799:365, pl. 137, fig. 4. - Illiger, 1806:231. - Schönner, 1808:326; 1843:411. - Duponchel, 1825:38, pl. 1, fig. 6. - Lacordaire, 1842:439. - Sturm, 1843:304. - Crotch, 1876:529. - Gemminger \& Harold, 1876:3715. - Kuhnt, 1908a:82, 85; 1909:35; 1911:26. Curran, 1941:283. - Deelder, 1942:66. - Blackwelder, 1945:461. Zimsen, 1964:114. - Alvarenga, 1977:105 (syn.).

varius Gmelin, 1790:1727. - Lacordaire, 1842:439 (syn.).

pustulatus Herbst, 1799:364, pl. 137, fig. 3. - Lacordaire, 1842:439 (syn.). jucundus Mader, 1942d:163, 187. Tp. ? - (Peru); 1944:115. Distr.: Peru.

kuhnti Deelder, 1942:64, fig. 1d. Tp.: RNHL - (Peru). Distr.: Peru. laetus Deelder, 1942:65, fig. 1e. Tp.: RNHL - (Peru). Distr.: Peru.

var. nigrofasciatus Deelder, 1942:65. Tp.: RNHL - (Brasil, Amazonas). Distr.: Brasil, região amazônica.

latreillei latreillei Lacordaire, 1842:461. Tp. ? - (Bolívia, Chiquitos). - Crotch, 1876:536. - Gemminger \& Harold, 1876:3713 (Cypherotylus). - Kuhnt, 1908a: 100 (Erotylus); 1909:38; 1911:24. - Mader, 1942c:566. - Blackwelder, 1945:461. Distr.: Bolívia.

latreillei normalis Mader, 1942c:566. Tp. ? - (Brasil, Est. S. Paulo, Indiana). Distr.: Brasil, região sudeste.

longulus Guérin, 1956:60, fig. 22. Tp. CDSP exam. aut. - (Bolívia, Yungas del Palmar). Distr.: Bolívia.

loratus Erichson, 1847:176. Tp.: ZMHU exam. aut. - (Peru). - Crotch, 1876:528. - Gemminger \& Harold, 1876:3714. - Dorhn, 1883:104. - Kuhnt, 1908a: 76, 81; 1909:34; 1911:24. - Deelder, 1942:65. - Mader, 1942d:161; 1944:112. Distr.: Peru.

var. tenuecinctus Kuhnt, 1908a:76, 81. Tp.: ZMHU exam. aut. - (Peru); 1909:34; 1911:24. - Mader, 1942d:161; 1944:112. Distr.: Peru.

luteotaeniatus Kuhnt, 1908a:76, 82. Tp ? (São Paulo ?); 1909:34; 1911:24. Blackwelder, 1945:461. Distr.: Brasil. 
margineguttatus Crotch, 1876:526. Tp.: MCZC exam. aut. - (Amazonas). Gemminger \& Harold, 1876:3714. - Kuhnt, 1908a:82, 83; 1909:35; 1911:24. - Deelder, 1942:65. - Mader, 1942d:162; 1944:113. - Blackwelder, 1945:461. - Guérin, 1948:15. Distr.: Peru. Bolívia. Brasil, região amazônica.

var. integer Kuhnt, 1908a:83. Tp. ? - (Peru, Marcapata); 1909:35; 1911:24. - Mader, 1942d:162; 1944:114. - Blackwelder, 1945:461. Distr.: Peru. Bolívia.

marginemaculatus Crotch, 1876:527. Tp.: MCZC exam. aut. - (Equador). Gemminger \& Harold, 1876:3714. - Kuhnt, 1908a:83; 1909:35; 1911:24. - Blackwelder, 1945:461. Distr.: Equador.

melanostictus Crotch, 1876:533. Tp.: MCZC exam. aut. - (Amazonas). - Gemminger \& Harold, 1876:3714. - Kuhnt, 1908a:99; 1909:38; 1911:24. Mader, 1942d:164; 1944:116. - Blackwelder, 1945:461. Distr.: Panamá. Peru. Brasil, região amazônica.

mirabilis Kuhnt, 1908a:72, 73. Tp.: ZMHU exam. aut. - (Brasil); 1909:34, pl. 2, fig. 9; 1911:24. - Blackwelder, 1945:461. - Guérin, 1948:15. Distr.: Brasil, região amazônica.

mutatus Mader, 1942c:566. Tp. ? - (Brasil, Amazonas, S. Paulo de Olivença). Distr.: Brasil, região amazônica.

nautae Crotch, 1876:528. Tp.: MCZC exam. aut. - (Nauta). - Gemminger \& Harold, 1876:3714. - Kuhnt, 1908a:88; 1909:36; 1911:24. - Blackwelder, 1945:461. Distr.: Peru.

neglectus Mader, 1942d:162, 186. Tp. ? - (Peru); 1944:112, 114. Distr.: Peru. Bolívia.

nemo Guérin, 1956:58. Distr.: Brasil, região amazônica.

tripartitus; Guérin, 1949, nec Kuhnt, 1908. - Guérin, 1949a:232, fig. 4. Tp.: CDSP exam. aut. - (Brasil, Amazonas, Maués); 1956:58 (nom. praeoc.).

nigrocinctus Kuhnt, 1908a:87. Tp.: ZMHU exam. aut. - (Peru); 1909:35, pl. 2, fig. 11; 1911:24. - Mader, 1942d:163; 1944:114. - Blackwelder, 1945:461. Distr.: Equador. Peru. Brasil, região amazônica.

nigromarmoratus Mader, 1942c:564. Tp.: MGFM exam. aut. - (Equador, Riobamba). Distr.: Equador.

nigronotatus Gorham, 1888:102, pl. 6, fig. 1. Tp.: NHML exam. aut. - (Panamá, Bugaba, volcán de Chiriquí). - Kuhnt, 1908a:98; 1909:37; 1911:24. Deelder, 1942:65. - Blackwelder, 1945:461. Distr.: Panamá.

oblitus Guérin, 1956:59, fig. 21. Tp.: CDSP exam. aut. - (Brasil, Amazonas, João Pessoa, rio Juruá). Distr.: Brasil, região amazônica.

ochraceus Guérin, 1946b:367, fig. 3. Tp.: CDSP exam. aut. - (Brasil, Est. de São Paulo, Indiana); 1948:15. Distr.: Brasil, região sudeste. 
olivieri Lacordaire, 1842:463. Tp. ? - (Bolívia, Guarayos). - Erichson, 1847:176; 1848:578. - Kirsch, 1876:108. - Crotch, 1876:530. - Gemminger \& Harold, 1876:3713 (Cypherotylus). - Kuhnt, 1908a:99 (Erotylus); 1909:38, pl. 1, fig. 1; 1911:24. - Deelder, 1942:65. - Mader, 1942d:164; 1944:115. Blackwelder, 1945:461. - Guérin, 1948:15. Distr.: Peru. Bolívia. Guiana. Brasil, região amazônica.

onagga Lacordaire, 1842:427. Tp. ? - (Colômbia). - Crotch, 1876:532. - Gemminger \& Harold, 1876:3714. - Kuhnt, 1908a:90; 1909:36, pl. 2, fig. 10; 1911:24. - Blackwelder, 1945:461. Distr.: Costa Rica. Colômbia.

var. quinquefasciata Kuhnt, 1908a:91. Tp. ? - [Localidade não citada]; 1909:36; 1911:24. - Blackwelder, 1945:461. Distr.: ?

papulosus Lacordaire, 1842:436. Tp. ? - (Caiena). - Crotch, 1876:530. Gemminger \& Harold, 1876:3714. - Kuhnt, 1908a:79, 82; 1909:35; 1911:24. - Blackwelder, 1945:461. Distr.: Guiana Francesa.

paraensis Alvarenga, 1976:511. Distr.: Brasil, região amazônica.

bicinctus; Guérin, 1948, nec Olivier, 1807. - Guérin, 1948:13, fig. 3. Tp.: MNRJ exam. aut. - (Pará, Santarém). - Alvarenga, 1976:511 (nom. praeoc.).

parcepunctatus Crotch, 1876:535. Tp.: MCZC exam. aut. - (Equador). - Gemminger \& Harold, 1876:3714. - Kuhnt, 1908a:100; 1909:38; 1911:25. Mader, 1942d:165; 1944:117. - Blackwelder, 1945:461. Distr.: Equador. Peru.

pardalis Erichson, 1848:578. Tp.: ZMHU exam. aut. - (Guiana). - Crotch, 1876:536. - Gemminger \& Harold, 1876:3714. - Kuhnt, 1908a:80, 82; 1909:35; 1911:25. - Blackwelder, 1945:461. Distr.: Guiana.

parvus Kuhnt, 1908a:74, 81. Tp. ? - (Peru, Marcapata); 1909:34; 1911:25. Mader, 1942d:162; 1944:113. - Blackwelder, 1945:461. Distr.: Peru.

peregrinus Mader, 1942d:164, 187. Tp.: DEIB - (Peru, Callanga); 1944:116. Distr.: Peru.

permutatus Kuhnt, 1908a:70, 74. Tp. ? - (Brasil); 1909:34; 1911:25. - Bruch, 1914:383. - Deelder, 1942:66. - Blackwelder, 1945:461. - Guérin, 1948: 15. Distr.: Brasil. Argentina.

peruvianus Crotch, 1876:527. Tp.: MCZC exam. aut. - (Peru). - Gemminger \& Harold, 1876:3714. - Kuhnt, 1908a:74, 81, 83; 1909:34; 1911:25. Brèthes, 1920:48. - Deelder, 1942:66. - Mader, 1942d:162; 1944:113. Blackwelder, 1945:461. - Guérin, 1948:15. Distr.: Equador. Peru. Bolívia. Brasil, região amzônica.

picturatus Crotch, 1876:529. Tp.: MCZC exam. aut. - (Equador). - Gemminger \& Harold, 1876:3715. - Kuhnt, 1908a:83, 85; 1909:35; 1911:25. - Blackwelder, 1945:461. Distr.: Equador.

pretiosus Perty, 1832:111, pl. 22, fig. 9. Tp. ? - (Brasil, rio Negro). - Lacordaire, 1842:440. - Crotch, 1876:529. - Gemminger \& Harold, 1876:3715. - 
Kuhnt, 1908a:90; 1909:36; 1909:25. - Bruch, 1914:383. - Deelder, 1942: 66. - Blackwelder, 1945:461. -Guérin, 1948:15. Distr.: Venezuela. Guiana Francesa. Brasil, região amazônica. Argentina.

var. geometricus Guérin, 1956:58. Tp.: CDSP exam. aut. - (Brasil, Amazonas, Borba). Distr.: Brasil, região amazônica.

var. impunctatus Kuhnt, 1908a:90. Tp.: ZMHU exam. aut. -(Brasil); 1909:36, 1911:25. - Bruch, 1914:383. - Blackwelder, 1945:461. - Guérin, 1948: 15. Distr.: Brasil, região amazônica.

var. infasciatus Kuhnt, 1910:268. Tp. ? - (Argentina, Misiones); 1911:25. Bruch, 1914:383. - Blackwelder, 1945:461. Distr.: Argentina.

propinquus Kuhnt, 1908a:77, 82. Tp.: ZMHU exam. aut. - [Localidade não citada]; 1908b:633; 1909:34; 1911:25. - Blackwelder, 1945:461. - Guérin, 1948:15. Distr.: Brasil, região amazônica.

pseudomelanostictus Guérin, 1946b:368, fig. 4. Tp.: CDSP exam. aut. - (Brasil, Amazonas, Benjamin Constant). Distr.: Brasil, região amazônica.

pulcher Mader, 1942c:563. Tp.: MGFM exam. aut. - (Colômbia, Umbria, rio Guines). Distr.: Colômbia.

quadricruentatus Alvarenga, 1976:511. Distr.: Brasil, região amazônica.

quadrimaculatus Guérin, 1948 nec Duponchel, 1825. - Guérin, 1948:15, fig. 4. Tp.: MNRJ exam. aut. - (Brasil, Amazonas, Manicoré). - Alvarenga, 1976:511 (nom.praeoc.).

reichei Guérin-Méneville, 1841a:117. Tp.: MCZC exam. aut. - (Brasil). Lacordaire, 1842:431. - Crotch, 1876:534. - Gemminger \& Harold, 1876:3715. - Kuhnt, 1908a:94; 1909:36; 1911:25. - Blackwelder, 1945: 461. Distr.: Bolívia. Brasil, região central.

rudepunctatus Crotch, 1876:527. Tp.: MCZC exam. aut. - (Amazonas). Gemminger \& Harold, 1876:3715. - Kuhnt, 1908a:82, 84; 1909:35; 1911:25. - Mader, 1942d:162; 1944:113, 115. - Blackwelder, 1945:461. Distr.: Colômbia. Peru. Brasil, região amazônica.

sanguinans Kuhnt, 1908a:88. Tp. ? - (Brasil, Amazonas); 1909:36; 1911:25. Blackwelder, 1945:461. - Guérin, 1948:16. Distr.: Brasil, região amazônica.

scaphidomorphoides Crotch, 1876:532. Tp.:MCZC exam. aut. - (Amazonas). Gemminger \& Harold, 1876:3715. - Kuhnt, 1908a:92; 1909:36; 1911:25.

- Blackwelder, 1945:461. Distr.: Brasil, região amazônica.

scenicus Erichson, 1847:176. Tp.: ZMHU exam. aut. - (Peru). - Crotch, 1876: 529. - Gemminger \& Harold, 1876:3715. - Kuhnt, 1908a:89; 1909:36; 1911:25. - Brèthes, 1920:48. - Deelder, 1942:66. - Mader, 1942d:162; 1944:114. - Blackwelder, 1945:461. Distr.: Peru.

schenklingi Kuhnt, 1908a:82, 84. Tp.: ZMHU exam. aut. - (Amazonas); 1909:35; 1911:25. - Blackwelder, 1945:461. Distr.: Brasil, região amazônica. sericeus Delkeskamp, 1957:97, 100, 101, figs 1, 2. Tp.: ZSBS exam. aut. - 
(Bolívia, Sarampiuni, San Carlos). Distr.: Bolívia.

singularis Kirsch, 1876:107. Tp.: SMTD exam. aut. - (Peru, Sarayacu). Gemminger \& Harold, 1876:3715. - Kuhnt, 1908a:89; 1909:36; 1911:25.

- Mader, 1942d:163; 1944:115. - Blackwelder, 1945:461. Distr.: Peru.

spectrum Thomson, 1856:476, pl. 23, fig. 7. Tp.: MNHN (Amazonas). - Crotch, 1876:572. - Gemminger \& Harold, 1876:3715. - Kuhnt, 1908a:80, 82; 1909:35; 1911:25. - Deelder, 1942:66. - Mader, 1942d:163; 1944:115. Blackwelder, 1945:461. - Guérin, 1948:16. - Delkeskamp, 1957:97.

Distr.: Peru. Bolívia. Brasil, região amazônica.

aterrimus Kirsch, 1876:107. - Kuhnt, 1908a:80 (syn.).

staudingeri Mader, 1935:241. Tp.: MGFM exam. aut. - (Peru, Oxapampa, Perene); 1942d:161; 1944:112. - Blackwelder, 1945:461. Distr.: Peru.

subreticulatus Guérin-Méneville, 1841a:115. Tp.: MNHN exam. aut. - (Brasil).

- Lacordaire, 1842:443. - Erichson, 1847:176. - Crotch, 1876:533. -

Gemminger \& Harold, 1876:3715. - Kuhnt, 1908a:97; 1909:37; 1911:25.

- Deelder, 1942:66. - Mader, 1942d:164; 1944:115. - Blackwelder, 1945: 461. - Guérin, 1948:16. - Delkeskamp, 1957:97. Distr.: Equador. Peru. Bolívia. Brasil, região amazônica.

superbus Guérin, 1956:58, fig. 20. Tp.: CDSP exam. aut. - (Peru, Tingo Maria). Distr.: Peru.

taeniatus Latreille, 1813:9, pl. 1, fig. 1. Tp. ? - (Peru). - Duponchel, 1825:39, pl. 1, fig. 9. - Lacordaire, 1842:428. - Guérin-Méneville, 1855:611. Crotch, 1876:526. - Gemminger \& Harold, 1876:3715. - Kuhnt, 1908a:75, 81; 1909:34; 1911:25. - Deelder, 1942:66. - Blackwelder, 1845:461.

Distr.: Colômbia. Peru.

fulgurator Lacordaire, 1842:427. - Crotch, 1876:526 (syn.). sanguiniceps Kuhnt, 1908a:75, 81. - Delkeskamp, 1939:30 (syn.). terminalis Kuhnt, 1908a:99. Tp. ? - (Brasil, rio Juruá); 1909:38; 1911:25. Blackwelder, 1945:461. Distr.: Brasil, região amazônica.

theodori Crotch, 1876:535. Distr.: Colômbia. Brasil, região amazônica.

unifasciatus; Lacordaire, 1842, nec Fabricius, 1798. - Lacordaire, 1842:433. Tp. ? - (Colombia). - Erichson, 1848:357. - Crotch, 1876:535 (nom. praeoc.).

trichromaticus Alvarenga, 1976:511. Distr.: Peru.

tricolor; Kuhnt, 1908, nec Fabricius, 1801. - Kuhnt, 1908a:237, 238 (Micrerotylus). Tp. ZMHU exam. aut. - (Peru); 1909:41; 1911:29. - Mader, 1942d:164, 188 (Erotylus); 1944:116. - Alvarenga, 1976:511 (nom. praeoc.).

tripartitus Kuhnt, 1908a:90. Tp.: ZMHU exam. aut. - (Brasil, Pará, Cametá); 1909:36; 1911:26. - Blackwelder, 1945:461. Distr.: Brasil, região amazônica. 
ustulatus Erichson, 1847:176. Tp.: ZMHU exam. aut. - (Peru). - Crotch, 1876:536. - Gemminger \& Harold, 1876:3715. - Kuhnt, 1908a:100; 1909:38; 1911:26. - Mader, 1942d:165; 1944:117. - Blackwelder, 1945: 461. Distr.: Peru. Bolívia.

varians Crotch, 1876:530. Tp.: MCZC exam. aut. - (Amazonas). - Fleutiaux, 1886:224. - Kuhnt, 1908a:86; 1909:35; 1911:26. - Deelder, 1942:66. Mader, 1942d:163; 1944:114. - Blackwelder, 1945:461. Distr.: Equador. Peru. Bolívia. Brasil, região amazônica.

variomaculatus Kuhnt, 1908a:77, 83. Tp.: ZMHU exam. aut. - (Bolívia, Yungas de La Paz); 1909:34; 1911:26. - Deelder, 1942:66. - Mader, 1942d:163; 1944:115. - Blackwelder, 1945:461. - Guérin, 1948:16. Distr.: Equador. Peru. Bolívia. Brasil, região amazônica.

var. completus Kuhnt, 1908a:78, 82. Tp. ? - [Localidade não citada]; 1909:35;

1911:26. - Mader, 1942d:163; 1944:113. - Blackwelder, 1945:461. Delkeskamp, 1957:97. Distr.: Bolívia.

var. niger Kuhnt, 1908a:77, 82. Tp.: ZMHU exam. aut. - [Localidade não citada]; 1909:34; 1911:26. - Deelder, 1942:66. - Mader, 1942d:163; 1944:115. - Blackwelder, 1945:461. Distr.: Equador. Peru.

var. paucipunctutus Kuhnt, 1908a:77. Tp. ? - [Localidade não citada]; 1909:

34; 1911:26. - Deelder, 1942:67. - Mader, 1942d:163. - Blackwelder, 1945:461. - Guérin, 1948:16. Distr.: Bolívia. Brasil, região amazônica. var. vittiger Kuhnt, 1908a:78. Tp. ? - [Localidade não citada]; 1909:35; 1911:26. - Deelder, 1942:67. - Mader, 1942d:163. - Blackwelder, 1945:461. Distr.: Bolívia.

vinculatus Lacordaire, 1842:426. Tp. ? (Colômbia). - Guérin-Méneville, 1855: 611. - Crotch, 1876:526. - Fleutiaux, 1886:224. - Kuhnt, 1908a:75, 81; 1909:34; 1911:26. - Deelder, 1942:67. - Blackwelder, 1945:461. Distr.: Colômbia.

voeti Lacordaire, 1842:429. Tp.: MCZC exam. aut. - (Bolívia, Mojos). - Kirsch, 1876:105. - Crotch, 1876:528. - Gemminger \& Harold, 1876:3715. Kuhnt, 1908a:74, 81; 1909:34; 1911:26. - Brèthes, 1920:48. - Deelder, 1942:67. - Mader, 1942d:162; 1944:112. - Blackwelder, 1945:461. Delkeskamp, 1957:97. Distr.: Peru. Bolívia.

ziczac Taschenberg, 1870:196. Tp. ? - (Colômbia). - Gemminger \& Harold, 1876:3715. - Kuhnt, 1908a:80, 81; 1909:35; 1911:26. - Mader, 1942d:162; 1944:113. - Blackwelder, 1945:461. Distr.: Colômbia. Peru. 


\section{Erotylina Curran, 1944}

Erotylina Curran, 1944:5 (Tgen. Erotylus leopardus Lacordaire, 1842:442, desig. orig. ). - Alvarenga, 1965:84.

atrotibialis Alvarenga, 1976:512. Distr.: Peru. Bolívia., Brasil, região amzônica. nigrotibialis; Crotch, 1876, nec Demay 1838. - Crotch, 1876:535 (Erotylus).

Tp.: MCZC exam. aut. - (Amazonas). - Gemminger \& Harold, 1876:3714. - Kuhnt, 1908a:98; 1909:37; 1911:24. - Deelder, 1942:65. - Mader, 1942d:164; 1944:116. - Blackwelder, 1945:461. - Guérin, 1948:15. - Curran, 1944:6, 8 (Erotylina). - Alvarenga, 1976:512 (nom.praeoc.).

bardia Curran, 1944:6, 7, fig. 10. Tp.: AMNH - (Peru, rio Abujas). Distr.: Peru. bassleri Curran, 1944:6, 12, fig. 16. Tp.: AMNH - (Peru, médio rio Ucayali). Distr.: Peru.

buqueti Lacordaire, 1842:445 (Erotylus). Tp. ? - (Brasil). - Crotch, 1876:526. Gemminger \& Harold, 1876:3713. - Kuhnt, 1908a:92; 1909:36; 1911:22. - Bruch, 1914:382. - Deelder, 1942:63. - Blackwelder, 1945:460. - Guérin, 1948:15. - Curran, 1944:6, 14, fig. 19 (Erotylina). Distr.: Brasil, regiões sudeste e sul. Argentina.

connectens Crotch, 1876:531 (Erotylus). Tp.: MCZC exam. aut. - (Brasil).

Gemminger \& Harold, 1876:3713. - Kuhnt, 1908a:95; 1909:37; 1911:22.

- Bruch, 1914:382. - Blackwelder, 1945:460. - Curran, 1944:6, 9, fig. 13 (Erotylina). Distr.: Brasil, regiões sudeste e sul. Argentina.

dichromostigma Guérin-Méneville, $1841 \mathrm{a}: 115$ (Erotylus). Tp.: MNHN exam. aut. - (Brasil central). - Lacordaire, 1842:441. - Crotch, 1876:531. -Gemminger \& Harold, 1876:3714. - Kuhnt, 1908a:95; 1909:37; 1911:22. - Bruch, 1914:383. - Blackwelder, 1945:460. - Delkeskamp, 1957:97 (Erotylina).

Distr.: Brasil, região central. Bolívia. Argentina.

dura Curran, 1944:6, 8, fig. 11. Tp.: AMNH - (Brasil, Amazonas, Tefé). Distr.: Brasil, região amazônica.

ecuadorica Crotch, 1876:530 (Erotylus). Tp.: MCZC exam. aut. - (Equador). Gemminger \& Harold, 1876:3714. - Kuhnt, 1908a:89; 1909:36; 1911:22

- Mader, 1942d:163; 1944:114. - Blackwelder, 1945:460. - Alvarenga, 1977: 104 (Erotylina). Distr.: Equador. Peru.

flavangula Crotch, 1876:535 (Erotylus). Tp.: MCZC exam. aut. - (Amazonas). Gemminger \& Harold, 1876:3714. - Kuhnt, 1908a:98; 1909:37; 1911:22. - Blackwelder, 1945:460. - Alvarenga, 1977:104 (Erotylina). Distr.: Brasil, região amazônica.

foveata Kuhnt, 1908a:95 (Erotylus). Tp.: ZMHU exam. aut. - [Localidade não citada]; 1909:37; 1911:22. - Blackwelder, 1945:460. - Curran, 1944:6, 10, fig. 14 (Erotylina). Distr.: Suriname. 
geminata Crotch, 1876:534 (Erotylus). Tp.: MCZC exam. aut. - (Amazonas). Gemminger \& Harold, 1876:3714. - Kuhnt, 1908a:99; 1909:37; 1911:22. - Blackwelder, 1945:460. - Guérin, 1948:15. - Alvarenga, 1977:104 (Erotylina). Distr.: Brasil, região amazônica.

gemmata Fabricius, 1792:38 (Erotylus). Tp.: ZMCD - (Caiena); 1801:5. - Herbst, 1799:361, pl. 137, fig. 1. - Illiger, 1806:231. - Olivier, 1807:471, pl. 2, fig. 14. - Schöenherr, 1808:327. - Duponchel, 1825:39, pl. 1, fig. 7. Lacordaire, 1842:437. - Crotch, 1876:533. - Gemminger \& Harold, 1876:3714. - Kuhnt, 1908a:99; 1909:37; 1911:22. - Deelder, 1942:64. Blackwelder, 1945:460. - Zimsen, 1964:114. - Alvarenga, 1977:104 (Erotylina). Distr.: Guiana Francesa. Brasil, região amazônica.

ghiliani Guérin-Méneville, 1855:611 (Erotylus). Tp.: MNHN exam. aut. - (Equador, Napo). - Crotch, 1876:537. - Gemminger \& Harold, 1876:3714. Kuhnt, 1908a:79, 82; 1909:35; 1911:22. - Blackwelder, 1945:460. Alvarenga, 1977:104 (Erotylina). Distr.: Equador.

helopioides Duponchel, 1825:40, pl. 1, fig. 11 (Erotylus). Tp.: MNHN exam. aut. - (Brasil, Minas Gerais). - Lacordaire, 1842:447. - Crotch, 1876:534. - Gemminger \& Harold, 1876:3714. - Kuhnt, 1908a:92; 1909:36; 1911:23.

- Blackwelder, 1945:460. - Guérin, 1948:15. - Curran, 1944:6, 13 (Erotylina). Distr.: Brasil, regiões sudeste e central.

conjugens Kuhnt, 1908a:93 (Erotylus). - Curran, 1944:13 (syn.).

herpestes Lacordaire, 1842:445 (Erotylus). Tp.: MCZC exam. aut. - (Colômbia).

- Crotch, 1876:532. - Gemminger \& Harold, 1876:3714. - Kuhnt, 1908a:

97; 1909:37; 1911:23. -Blackwelder, 1945:460. - Alvarenga, 1977:104 (Erotylina). Distr.: Colômbia.

imperfecta Crotch, 1876:533 (Erotylus). Tp.: MCZC exam. aut. - (Equador).

Gemminger \& Harold, 1876:3714. - Kuhnt, 1908a:97; 1909:37; 1911:23.

- Blackwelder, 1945:460. - Alvarenga, 1977:104 (Erotylina). Distr.: Equador.

intermedia Crotch, 1876:531 (Erotylus). Tp.: MCZC exam. aut. - (Colômbia). Gemminger \& Harold, 1876:3714. - Kuhnt, 1908a:95; 1909:37; 1911:24.

- Blackwelder, 1945:460. - Curran, 1944:6, 10 (Erotylina). Distr.: Colômbia. Brasil, região amazônica.

jaspidea Erichson, 1847:176 (Erotylus). Tp. ? - (Peru). - Crotch, 1876:531. Gemminger \& Harold, 1876:3714. - Kuhnt, 1908a:95; 1909:37, pl. 2, fig. 12; 1911:24. - Bruch, 1914:383. - Deelder, 1942:64. - Mader, 1942d:163; 1944:115. - Blackwelder, 1945:460. - Curran, 1944:10 (Erotylina).

Alvarenga, 1977:105. Distr.: Peru. Bolívia. Paraguai. Brasil. Argentina. var. minor Kuhnt, 1908a:95. - Curran, 1944:10 (syn.). var. basilianus Kuhnt, 1908a:96. - Curran, 1944:10 (syn.).

pustulatus; Duponchel, 1825, nec Fabricius, 1781. - Duponchel, 1825:39, pl. 1, fig. 8 (Erotylus). Tp.: MNHN exam. aut. - (Brasil). - Lacordaire, 1842:437. - Crotch, 1876:530. - Gemminger \& Harold, 1876:3715. - 
Kuhnt, 1908a:94; 1909:37; 1911:25. - Bruch, 1914:383. - Blackwelder, 1945:461. - Curran, 1944:10 (Erotylina). - Delkeskamp, 1957:97. Alvarenga, 1977:105 (nom.praeoc.).

lacordairei Lacordaire, 1842:446 (Erotylus). Tp.: MCZC exam. aut. - (Rio de Janeiro). - Guérin-Méneville, 1855:611. - Crotch, 1876:534. - Kuhnt, 1908a:93; 1909:36; 1911:23. - Blackwelder, 1945:460. - Guérin, 1948:15. - Curran, 1944:6, 13, fig. 18 (Erotylina). Distr.: Brasil, região sudeste.

leoparda Lacordaire, 1842:442 (Erotylus). Tp. ? - (México, Yucatán). - Crotch, 1876:531. - Gemminger \& Harold, 1876:3714. - Gorham, 1888:102, pl. 5, fig. 17-20. - Kuhnt, 1908a:96; 1909:37; 1911:24. - Deelder, 1942:65. - Blackwelder, 1945:461. - Curran, 1944:6, 9, fig. 12 (Erotylina). Distr.: México e América Central.

confluens Crotch, 1876:531 (Erotylus). - Gorham, 1888:102 (syn.).

maculiventris Lacordaire, 1842:444 (Erotylus). Tp. ? - (Colômbia). - Erichson, 1847:176. - Guérin-Méneville, 1855:611. - Crotch, 1876:535. - Gemminger \& Harold, 1876:3714. - Kuhnt, 1908a:98; 1909:37; 1911:24. Bréthes, 1920:48. - Deelder, 1942:65. - Mader, 1942d:164; 1944:116. Blackwelder, 1945:461. - Guérin, 1948:15. - Curran, 1944:6, 7, fig. 9 (Erotylina). - Delkeskamp, 1957:97. Distr.: Panamá. Colômbia. Equador. Peru. Bolívia. Brasil, região amazônica.

marshami Lacordaire, 1842:425 (Erotylus). Tp.: MCZC exam. aut. - (Caiena). Crotch, 1876:526. - Gemminger \& Harold, 1876:3714. - Kuhnt, 1908a:92; 1909:36; 1911:24. - Blackwelder, 1945:461. - Alvarenga, 1977:104 (Erotylina). Distr.: Guiana Francesa.

microguttata Kuhnt, 1908a:96 (Erotylus). Tp.: ZMHU exam. aut. - (Brasil, Mato Grosso); 1909:37; 1911:24. - Blackwelder, 1945:461. - Guérin, 1948:15. -_ Alvaenga, 1977:104 (Erotylina).

multiguttata Lacordaire, 1842:441 (Erotylus). Tp.: MNHN exam. aut. - (Bolívia). - Blanchard, 1843, pl. 25, fig. 8. - Crotch, 1876:531. - Gemminger \& Harold, 1876:3714. - Kuhnt, 1908a:98; 1909:37; 1911:24. - Blackwelder, 1945:461. - Curran, 1944:6, 8 (Erotylina). Distr.: Peru. Bolívia. Paraguai. nicaraguae Crotch, 1873b: 148 (Erotylus). Tp.: MCZC exam. aut. (Nicarágua, Chontales, S. Domingo); 1876:532. - Gemminger \& Harold, 1876:3714. - Gorham, 1888:102, pl. 5, fig. 21. - Kuhnt, 1908a:96; 1909:37; 1911:24. - Blackwelder, 1945:461. - Curran, 1944:9 (Erotylina). Distr.: Nicarágua. scita Curran, 1944:6, 12, fig. 17. Tp.: AMNH - (Equador, Zamora). Distr.: Equador.

scutellata Kuhnt, 1908a:97 (Erotylus). Tp. ? - (Colômbia); 1909:37; 1911:25. Blackwelder, 1945:461. - Curran, 1944:6, 11, fig. 15 (Erotylina). Distr.: Colômbia.

teresae Alvarenga, 1976:512. Distr.: Colômbia.

rufipes; Crotch, 1876, nec Fabricius, 1781. - Crotch, 1876:535 (Erotylus). 
Tp. ? - (Colômbia, Bogotá). - Gemminger \& Harold, 1876:3715. -

Kuhnt, 1908a:98; 1909:37; 1911:25. - Blackwelder, 1945:461. -

Curran, 1944:6, 7 (Erotylina). - Alvarenga, 1976:512 (nom.praeoc.) toxophora Lacordaire, 1842:432 (Erotylus). Tp.: MNHN exam. aut. - (Bolívia).

- Crotch, 1876:534. - Gemminger \& Harold, 1876:3715. - Kuhnt, 1908a: 94; 1909:36; 1911:25. - Deelder, 1942:66. - Mader, 1942d:164; 1944:116.

- Blackwelder, 1945:461. - Delkeskamp, 1957:97. - Alvarenga, 1977:104

(Erotylina). Distr.: Peru. Bolívia. Brasil, região amazônica.

toxographus Erichson, 1847:176. - Crotch, 1876:534. - Kuhnt, 1908a:94 (syn.).

vixen Curran, 1944:6, 11. Tp.: AMNH - (Brasil, Amazonas, Tefé). Distr.: Brasil, região amazônica.

\section{Gibbifer Voet, 1778}

Voet, 1778:62 (Tgen. Gibbifer tigrinus Voet, 1778:62, monotipia). - Alvarenga, 1970:45.

Hypselonotus Hope, 1841. - Hope, $1841 \mathrm{a}: 110$ (Tgen. Erotylus sphacelatus Fabricius, 1801, desig. orig.). - Curran, 1941:283. - Alvarenga, 1965:85 (nom.praeoc., Hahn, 1826).

Cypherotylus Crotch, 1873a:358 (Tgen. Chrysomela gibbosa Linnaeus, 1763, desig. orig.); 1873b:148; 1876:537. - Gorham, 1888:103. - Kuhnt, 1908a: 225; 1909:10, 38. - Deelder, 1942:52. - Boyle, 1956:93. - ALvarenga, 1970:45 (syn.).

adrianae Alvarenga, 1976:512. Distr.: Brasil, região sudeste.

apiatus; Lacordaire, 1842, nec Chevrolat, 1835. - Lacordaire, 1842:462 (Erotylus). Tp.: MCZC exam. aut. - (Rio de Janeiro). - Crotch, 1876:539 (Cypherotylus). - Gemminger \& Harold, 1876:3712. - Kuhnt, 1908a: 230, 235: 1909:39; 1911:27. - Bruch, 1914:383. - Mader, 1935:244. Deelder, 1942;67. Blackwelder, 1945:461. - Guérin, 1948:17. - Alvarenga, 1976:512 (nom.praeoc.).

var. foraminosus Lacordaire, 1842:463 (Erotylus). Tp. ? - (Brasil). Crotch, 1876:539 (Cypherotylus). - Gemminer \& Harold, 1876:3712. - Kuhnt, 1908a:230, 236;1909:39; 1911:27. - Bruch, 1914:383. - Blackwelder, 1945:461. - Guérin, 1948:17. Distr.: Brasil, região sul. Argentina.

aeneoniger Crotch, 1876:539 (Cypherotylus). Tp.: MCZC exam. aut. - (Equador). - Fleutiaux, 1886:224. - Kuhnt, 1908a:231, 235; 1909:39; 1911:26. Blackwelder, 1945:461. Distr.: Equador.

alutaceus Gorham, 1888:107, pl. 6, fig. 10 (Cypherotylus). Tp.: NHML exam. aut. - (México, Sonora); 1898:256. - Kuhnt, 1908a:232, 236; 1909:40; 1911:26. - Blackwelder, 1945:461. Distr.: México.

annulipes Guérin-Méneville, 1841a:115 (Hypselonotus). Tp.: MNHN exam. aut. 
- (Oeste Brasil). - Lacordaire, 1842:461 (Erotylus). - Crotch, 1876:539 (Cypherotylus). - Gemminger \& Harold, 1876:3712. - Kuhnt, 1908a:227, 234; 1909:39; 1911:26. - Bruch, 1914:383. - Blackwelder, 1945:461. Distr.: Bolívia. Brasil, região central. Argentina.

var. nigricollis Kuhnt, 1908a:228, 234. Tp. ? - (Bolívia); 1909:39; 1911:26. - Blackwelder, 1945:461. Distr.: Bolívia.

anthracinus Gorham, 1888:104 (Cypherotylus). Tp.: NHML exam. aut. - (Peru, Chanchamayo). - Kuhnt, 1908a:230, 234; 1909:39; 1911:27. - Deelder, 1942:67. - Mader, 1942d:166; 1951:198. - Blackwelder, 1945:461. Distr.: Peru. Bolívia.

apicalis Crotch, 1876:540 (Cypherotylus). Tp.: MCZC exam. aut. - (Colômbia). - Gemminger \& Harold, 1876:3712. - Kuhnt, 1908a:231, 236; 1909:39; 1911:27. - Blackwelder, 1945:461. Distr.: Colômbia.

armillatus Erichson, 1847:176 (Erotylus). Tp. ? - (Peru). - Crotch, 1876:538 (Cypherotylus). - Gemminger \& Harold, 1876:3713. - Kuhnt, 1908a:227, 234; 1909:39; 1911:27. - Deelder, 1942:67. - Mader, 1942d:165; 1951: 198. - Blackwelder, 1945:461. Distr.: Peru. Bolívia.

ater Kirsch, 1876:109 (Erotylus). Tp.: SMTD exam. aut. - (Peru, Pozuzu). Gemminger \& Harold, 1987:3713. - Kuhnt, 1908a:230, 234 (Cypherotylus); 1909:39; 1911:27. - Mader, 1942d:166; 1951:198. - Blackwelder, 1945:461. - Guérin, 1948:17. Distr.: Peru. Bolíva. Brasil, região amazônica.

badeni Dohrn, 1883:103 (Cypherotylus). Tp. ? - (Amazônas). - Fleutiaux, 1886:224. - Kuhnt, 1908a:226, 233; 1909:38; 1911:27. - Mader, 1942d: 165; 1951:197. - Blackwelder, 1945:461. Distr.: Peru. Brasil, região amazônica .

banghaasi Mader, 1935:242, 270 (Cypherotylus). Tp.: MGFM exam. aut. [Equívoco na etiqueta de localidade, cita Brasil]. - Blackwelder, 1945:461. Distr.: Guatemala.

boisduvali Chevrolat, 1834, fasc. 4 (Erotylus). Tp. ? - (México). - Lacordaire, 1842:466. - LeConte, 1854:163. - Crotch, 1873a:358 (Cypherotylus); 1873b:148; 1876:540. - Gemminger \& Harold, 1876:3712. - Gorham, 1888:106, pl. 5, fig. 23. - Kuhnt, 1908a:232, 236; 1909:40; 1911:27. Deelder, 1942:67. - Blackwelder, 1945:461. - Peterson, 1951:190, figs C53, B. - Boyle, 1956:93. Distr.: México. Guatemala.

bolivianus Mader, 1942c:568 (Cypherotylus). Tp.: MGFM exam. aut. - (Bolívia, Santa Cruz). Distr.: Bolívia.

borgmeieri Alvarenga, 1976:512. Distr.: Brasil, regiões sudeste e sul.

annulatus; Lacordaire, 1842, nec Germar, 1824. - Lacordaire, 1842:456 (Erotylus). Tp.: MCZC exam. aut. - (Brasil, Bahia). - Crotch, 1876:538 (Cypherotylus). - Gemminger \& Harold, 1876:3712. - Kuhnt, 1908a: 227, 234; 1909:39; 1911:26. - Deelder, 1942:67. - Blackwelder, 
1945:461. - Guérin, 1948:17. - Alvarenga, 1976:512 (nom.praeoc.). var. neglectus Kuhnt, 1908a:227, 234; 1909:39; 1911:26. - Blackwelder, 1945:461. Distr.: Venezuela.

californicus Lacordaire, 1842:467 (Erotylus). Tp. ? - (U.S.A., Califórnia). LeConte, 1854:163. - Boyle, 1956:93, figs 61-67 (Cypherotylus). Distr.: U.S.A. e México.

aspersus Gorham, 1888:108, pl. 6, fig. 9 (Cypherotylus). Tp.: NHML (México, Chihuahua, Pinos Altos). - Kuhnt, 1908a:232, 236; 1909:40, pl. 1, fig. 3; 1911:27. - Böving \& Craighead, 1930:39, pl. 41, figs A-C, E, G. (Biol.). - Pallister, 1955a:3. - Boyle, 1956:93 (syn.).

camelus Guérin-Méneville, 1841a:116 (Hypselonotus). Tp.: MCZC exam. aut. (Bolívia). - Lacordaire, 1842:452 (Erotylus). - Crotch, 1876:537 (Cypherotylus). - Gemminger \& Harold, 1876:3712. - Kuhnt, 1908a:228, 234; 1909:39; 1911:27. - Blackwelder, 1945:461. - Guérin, 1948:17. Distr.: Bolívia. Brasil, região amazônica.

costaricensis Gorham, 1888:105, pl. 6, fig. 7 (Cypherotylus). Tp.: NHML (Costa Rica). - Kuhnt, 1908a:232, 236; 1909:40; 1911:27. - Deelder, 1942:67. Blackwelder, 1945:461. Distr.: Costa Rica. Panamá.

debauvei Demay, 1838:23 (Erotylus). Tp. ? _(Guiana Francesa). - Lacordaire, 1842:457. - Crotch, 1876:538 (Cypherotylus). - Gemminger \& Harold, 1876:3712. - Gorham, 1888:103, pl. 6, figs 2, 2a. - Kuhnt, 1908a:227, 234; 1909:39; 1911:27. - Deelder, 1942:67. - Blackwelder, 1945:461. Guérin, 1948:17. Distr.: Panamá. Colômbia. Venezuela. Guiana Francesa. Bolívia. Brasil, região amazônica.

dromedarius Lacordaire, 1842:455 (Erotylus). Tp. ? - (Caiena). - Crotch, 1873b: 148 (Cypherotylus); 1876:538. - Gemminger \& Harold, 1876:3712. Gorham, 1888:103. - Kuhnt, 1908a:226, 233; 1909:38; 1911:27. - Deelder, 1942:67. - Mader, 1942d:201; 1951:198. - Blackwelder, 1945:461. Distr.: Da América Central até ao Brasil, região amazônica.

elevatus Fabricius, 1801:4 (Erotylus). Tp.: ZMCD - [Localidade não citada]. Illiger, 1806:230. - Schönherr, 1808:326. - Duponchel, 1825:167. Lacordaire, 1842:459. - Sturm, 1843:304. - Zimsen, 1964:114. - Crotch, 1876:538 (Cypherotylus). - Gemminger \& Harold, 1876:3712. Gorham, 1888:104, pl. 6, figs 4, 4a. - Kuhnt, 1908a:229, 235; 1909:39; 1911:27. Bruch, 1914:383 - Deelder, 1942:67. - Blackwelder, 1945:462. - Guérin, 1948:17. Distr.: Panamá. Colômbia. Guiana Francesa. Brasil. Argentina. fenestratus Gorham, 1888:107, pl. 5, fig. 22 (Cypherotylus). Tp.: NHML exam. aut. - (México, Refugio). - Kuhnt, 1908a:233, 237; 1909:40; 1911:27. Mader, 1935:244. - Deelder, 1942:67. - Blackwelder, 1945:462. - Pallister, 1955a:3. Distr.: México.

gaumeri Gorham, 1888:106, pl. 6, fig. 8 (Cypherotylus). Tp.: NHML exam. aut. - (México, Yucatán, Temax). - Kuhnt, 1908a:231, 236; 1909:40; 1911:27.

- Blackwelder, 1945:462. Distr.: México. 
gibbosus Linnaeus, 1763:10 (Chrysomela). Tp. ? - (Suriname). - Gronovius, 1763:606, pl. 14, fig. 5. - Hottuyn, 1766, pl. 74, fig. 5. - Herbst, 1783:51, pl. 23, fig. 3. - Fabricius, 1781 (Erotylus); 1787:91; 1792:36; 1801:4. Gmelin, 1790:1727. - Olivier, 1792:432; 1807:469, pl. 1, figs 4, a, b. Herbst, 1799:366, pl. 137, fig. 5. - Latreille, 1804:37. - Illiger, 1806:230. - Schönherr, 1808:326. - Duméril, 1823, pl. 21, fig. 13. - Duponchel, 1825:36, pl. 1, fig. 2. - Lacordaire, 1842:453. - Sturm, 1843:304. - Kirsch, 1876:108. - Crotch, 1876:538 (Cypherotylus). - Gemminger \& Harold, 1876:3713. - Gorham, 1888;103, pl. 6, figs 3, 3a. - Kuhnt, 1908a:228, 235; 1909:39 1911:27. - Curran, 1941:283 (Hypselonotus). - Deelder, 1942:67 (Cypherotylus). - Mader, 1942d: 166; 1951:199. - Blackwelder, 1945:462. - Guérin, 1948:17. Distr.: Nicarágua. Panamá. Suriname. Guiana Francesa. Equador. Peru. Brasil, região amazônica.

goryi Guérin-Méneville, 1841a:116 (Hypselonotus). Tp.: MNHN exam. aut. (Colômbia). - Lacordaire, 1842:464 (Erotylus). - Crotch, 1876:539 (Cypherotylus). - Gemminger \& Harold, 1876:3713. - Kuhnt, 1908a:232, 236; 1909:40; 1911:28. - Mader, 1935:270. - Deelder, 1942:67. - Blackwelder, 1945:462. Distr.: Colômbia.

gracilis Kuhnt, 1908a:226 (Cypherotylus). Tp.: ZMHU exam. aut. - (Peru, Chanchamayo); 1909:39, pl. 1, figs 4, 4a; 1911:28. - Mader, 1942d: 165; 1951:198. - Blackwelder, 1945:462. Distr.: Peru.

guatemalae Crotch, 1876:540 (Cypherotylus). Tp.: MCZC exam. aut. - (Guatemala). - Gemminger \& Harold, 1876:3713. - Gorham, 1888:105, pl. 6, fig. 6. - Kuhnt, 1908a:232, 237; 1909:40; 1911:28. - Mader, 1935:270. Deelder, 1942:67. - Blackwelder, 1945:462. Distr.: Guatemala.

hopfneri Mader, 1935:243, 271 (Cypherotylus). Tp.: MGFM exam. aut. - (Guatemala, La Conquista). - Blackwelder, 1945:462. Distr.: Guatemala.

impressopunctatus Crotch, 1873b:148 (Cypherotylus). Tp.: MCZC exam. aut. (Nicaraguá, Chontales, Santo Domingo); 1876:540. - Gemminger \& Harold, 1876:3713. - Gorham, 1888:104, pl. 6, fig. 5. - Kuhnt, 1908a:231, 236; 1909:40; 1911:28. - Blackwelder, 1945:462. Distr.: Nicaraguá. Panamá.

impuctatus Crotch, 1876:537 (Cypherotylus). Tp.: MCZC exam. aut. - (Amazonas). - Gemminger \& Harold, 1876:3713. - Kuhnt, 1908a:226, 233; 1909:38; 1911:28. - Blackwelder, 1945:462. - Guérin, 1948:17. Distr.: Brasil, região amazônica.

intercedens Kuhnt, 1908a:228, 235 (Cypherotylus). Tp. ? - (Veragua); 1909:39; 1911:28. - Blackwelder, 1945:462. Distr.: Panamá. Colômbia.

irroratus Kuhnt, 1908a:229, 234 (Cypherotylus). Tp. ? - (Peru); 1909:39; 1911: 28. - Mader, 1942d: 166; 1951:199. - Blackwelder, 1945:462. Distr.: Peru. jacquieri Lacordaire, 1842:451 (Erotylus). Tp. ? - (Caiena). - Crotch, 1876:537 (Cypherotylus). - Gemminger \& Harold, 1876:3713. - Kuhnt, 1908a:225, 223; 1909:38; 1911:28. - Deelder, 1942:68. - Blackwelder, 1945:462. 
Distr.: Suriname. Guiana Francesa.

maximus Crotch, 1876:537 (Cypherotylus). Tp.: MCZC exam. aut. - (Alto Amazonas). - Gemminger \& Harold, 1876:3713. - Kuhnt, 1908a:226, 233; 1909:38; 1911:28. - Mader, 1942d:165; 1951:197. - Blackwelder, 1945: 462. Distr.: Equador. Peru. Brasil, região amazônica.

miliaris Lacordaire, 1842:458 (Erotylus). Tp.: MCZC exam. aut. - (Bolívia). Crotch, 1876:538 (Cypherotylus). - Gemminger \& Harold, 1876:3713. Kuhnt, 1908a:229, 235; 1909:39; 1911:28. - Mader, 1942c:569; 1942d: 166; 1951:199. - Blackwelder, 1945:462. - Guérin, 1948:17. - Delkeskamp, 1957:97. Distr.: Colômbia. Peru. Bolívia. Brasil. região amazônica. obstinatus Mader, 1942c:567 (Cypherotylus). Tp.: MGFM exam. aut. - (Bolívia, Santa Cruz). Distr.: Bolívia.

ovalis Mader, 1942c:570 (Cypherotylus). Tp.: MGFM exam. aut. - (Guatemala, Panzos). Distr.: Guatemala.

patellatus Gorham, 1888:104 (Cypherotylus). Tp.: NHML exam. aut. - (Peru). Kuhnt, 1908a:230, 234; 1909:39; 1911:28. - Deelder, 1942:68. - Mader, 1942d:166; 1951:199. - Blackwelder, 1945:462. Distr.: Panamá. Equador. Peru.

seriatus Kuhnt, 1908a:229, 235 (Cypherotylus). Tp. ? - (Bolívia); 1909:39; 1911:28. - Buch, 1914:383. - Mader, 1942c:569. - Blackwelder, 1945: 462. Distr.: Bolívia. Argentina.

var.nigroterminalis Kuhnt, 1908a:229, 235. Tp. ? - (Bolívia); 1909:39; 1911:28. - Blackwelder, 1945:462. Bolívia.

sticticus Erichson, 1847:176 (Erotylus). Tp.: ZMHU exam. aut. -(Peru). - Crotch, 1876:540 (Cypherotylus). - Gemminger \& Harold, 1876:3713. - Kuhnt, 1908a:231; 1909:39; 1911:28. - Mader, 1942d:166; 1951:200. - Blackwelder, 1945:462. Distr.: Equador. Peru.

stillatus Kirsch, 1865:100 (Erotylus). Tp.: SMTD exam. aut. - (Colômbia, Bogotá). - Crotch, 1876:540 (Cypherotylus). - Gemminger \& Harold, 1876:3713. - Kuhnt, 1908a:231, 236; 1909:40; 1911:28. - Deelder, 1942: 68. - Mader, 1942d:166; 1951:200. - Blackwelder, 1945:462. Distr.: Colômbia. Peru.

tectiformis Kuhnt, 1910:268 (Erotylus). Tp.: MHNU exam. aut. - (Brasil, Mato Grosso); 1911:25. - Blackwelder, 1945:461. - Guérin, 1948:16. - Mader, 1938:17 (Cypherotylus). Distr.: Brasil, região amazônica.

tigrinus Voet, 1778:62, pl. 44, figs 1, 2. Tp. ? - [Localidade não citada]. Alvarenga, 1977:106. Distr.: Peru. Brasil. Argentina.

duponcheli Arrow, 1937:106 (Cypherotylus). Alvarenga, 1977:106 (syn.). sphacelatus; auctorum, nec Fabricius, 1801 (Tenebrionidae).

triangularis Guérin, 1949a:233, tig. 5 (Cypherotylus). Tp.: CDSP exam. aut. (Brasil, Pará, Santarém). Distr.: Brasil, região amazônica. 
variolosus Crotch, 1876:540 (Cypherotylus). Tp.: MCZC exam. aut. - (Equador). - Gemminger \& Harold, 1876:3713. - Kuhnt, 1908a:231, 236; 1909:39; 1911:28. - Blackwelder, 1945:462. Distr.: Equador.

vicinus Guérin-Méneville, $1841 \mathrm{a}: 116$ (Hypselonotus). Tp.: MNHN exam. aut. (México). - Lacordaire, 1842:465 (Erotylus). - Crotch, 1876:539 (Cypherotylus). - Gemminger \& Harold, 1876:3713. - Gorham, 1888:105, pl. 5, fig. 24. - Kuhnt, 1908a:232, 236; 1909:40; 1911:28. - Mader, 1935:244. - Deelder, 1942:68. - Blackwelder, 1945:462. Distr.: México. Guatemala. Nicarágua.

var. jansoni Crotch, 1873b:149. Tp.: ? - (Nicaraguá, Chontales, Santo Domingo); 1876:539. - Gemminger \& Harold, 1876:3713. - Gorham, 1888: 105. - Kuhnt, 1909:40; 1911:28. - Mader, 1935:244. - Deelder, 1942:68. - Blackwelder, 1945:462. Distr.: Nicarágua.

melanostigma Lacordaire, 1842:466 (Erotylus). - Crotch, 1876:539 (syn.). zebu Kirsch, 1876: 108 (Erotylus). Tp. ? - (Peru, Pozuzo). - Gemminger \& Harold, 1876:3715. - Kuhnt, 1908a:226, 233 (Cypherotylus); 1909:39; 1911:28. Mader, 1942d:165; 1951:198. - Blackwelder, 1945:462. - Guérin, 1948: 17. Distr.: Peru. Brasil, região amazônica.

\section{Barytopus Chevrolat, 1837}

Barytopus Chevrolat, 1837:425 (Tgen. Chrysomela gronovii Herbst, 1783:52, seg. Duponchel \& Chevrolat, 1844:505). - Duponchel \& Chevrolat, 1844:505. Alvarenga, 1965:86.

Micrerotylus Crotch, 1876:541 (Tgen. Chrysomela gronovii Herbst, 1783:52, desig. orig. ). - Gorham, 1888:108. - Kuhnt, 1908a:237; 1909:9, 40. - Deelder, 1942:51. - Alvarenga, 1965:82 (syn.).

dubitabilis Crotch, 1876:541 (Micrerotylus). Tp.: MCZC exam. aut. - (Amazonas). - Gemminger \& Harold, 1876:3715. - Kuhnt, 1908a:238; 1909:40, pl. 1, fig. 6; 1911:29. - Bruch, 1914:383. - Deelder, 1942:68. - Mader, 1942d:161; 1944:111. - Blackwelder, 1945:462. - Guérin, 1948:17. Distr.: Peru. Brasil. Argentina.

var. quinquepunctatus Kuhnt, 1908a:238. Tp. ? - (Peru, Chanchamayo); 1909:40; 1911:29. - Mader, 1942d:161. - Blackwelder, 1945:462. Distr.: Peru.

funerellus Crotch, 1876:541 (Micrerotylus). Tp.: MCZC exam. aut. - (Colômbia). - Gemminger \& Harold, 1876:3715. - Kuhnt, 1908a:238; 1909:41; 1911: 29. - Blackwelder, 1945:462. Distr.: Colômbia.

hesitans Crotch, 1876:541 (Micrerotylus). Tp.: MCZC exam. aut. - (Equador). Gemminger \& Harold, 1876:3716. - Kuhnt, 1908a:238; 1909:41; 1911:29. - Deelder, 1942:68. - Mader, 1942d:161; 1944:111. - Blackwelder, 1945: 462. Distr.: Equador. Peru. Brasil, região amazônica. 
heterogrammus Lacordaire, 1842:382 (Brachysphoenus). Tp.: MNHN exam. aut. - (Bolívia, Santa Cruz de la Sierra). - Crotch, 1876:541 (Micrerotylus). Gemminger \& Harold, 1876:3716. - Kuhnt, 1908a:238; 1909:41; 1911:29.

- Blackwelder, 1945:462. - Delkeskamp, 1957:97. Distr.: Bolívia.

laevigatus Deelder, 1942:68, fig. If (Micrerotylus). Tp.: RNHL - (Costa Rica). Distr.: Costa Rica.

lunulatus Olivier, 1792:435 (Erotylus). Tp. ? - (Suriname). - Crotch, 1876:541

(Micrerotylus). - Gemminger \& Harold, 1876:3716. - Gorham, 1888:108, pl. 6, fig. 13. - Kuhnt, 1908a:238; 1908b:633; 1909:41, pl. 1, fig. 5; 1911:29. - Mader, 1942d:161; 1944:111. - Blackwelder, 1945:462. Distr.:

Nicarágua. Panamá. Colômbia. Suriname. Guiana Francesa. Peru.

lugubris Lacordaire, 1842:383 (Brachysphoenus). - Crotch, 1876:541 (syn.). surinamensis Voet, 1778:43, pl. 33, fig. 1 (Pseudochrysomela). Tp. ? - (Suriname). - Alvarenga, 1977:105 (Barytopus). Distr.: Suriname. Guiana Francesa.

gronovii Herbst, 1783:52, pl. 23, fig. 4 (Chrysomela). Tp.: MNHN exam. aut. - [Localidade não citada]. - Gmelin, 1790: 1685. - Crotch, 1876:541 (Micrerotylus). - Gemminger \& Harold, 1876:3715. - Kuhnt, 1908a: 238; 1909:41; 1911:29. - Deelder, 1942:68. - Blackwelder, 1945:462. - Alvarenga, 1977:105 (syn.).

alternans Olivier, 1792:434 (Erotylus); 1807:472, pl. 1, figs 10a, b. Fabricius, 1792:39; 1801:7. - Herbst, 1799:369, pl. 137, fig. 8. - Illiger, 1806: 232. - Schönherr, 1808:238. - Duponchel, 1825:42, pl. 1, fig. 15. Lacordaire, 1842:379 (Brachysphoenus), - Guérin-Méneville, 1855: 611. - Crotch, 1876:541 (=Micrerotylus gronovii (Herbst, 1783)).

\section{Oligocorynus Chevrolat, 1837}

Oligocorynus Chevrolat, 1837:426 (Tgen. Erotylus cinctus Herbst, 1799:372, seg. Alvarenga, 1965:87) - Alvarenga, 1965:87.

Alloiotelus Hope, 1841:112 (Tgen. Erotylus discoideus Olivier, 1807, desig. orig.). - Curran, 1944:5. - Alvarenga, 1965:81 (syn.).

Zonarius Hope, 1841:111 (Tgen. Erotylus fasciatus Fabricius, 1801, desig. orig.). - Lacordaire, 1842:32, 468. - Chapuis, 1876:49, 64. - Crotch, 1876:542. Gorham, 1888:109. - Kuhnt, 1909:10, 41; 1910:251. - Deelder, 1942:52. Curran, 1944:5 - Alvarenga, 1965:90 (Tenebrionidae).

buckleyi Crotch, 1876:545 (Zonarius). Tp.: MCZC exam. aut. - (Equador). Gemminger \& Harold, 1876:3716. - Kuhnt, 1909:42. - 1910:254; 1911:29.

- Curran, 1941:284. - Blackwelder, 1945:462. Distr.: Equador.

cacicus Lacordaire, 1842:474 (Zonarius). Tp. ? (México, Orizaba). - Crotch, 1876:542. - Gemminger \& Harold, 1876:3716, - Gorham, 1888:109, pl. 6, fig. 14. - Kuhnt, 1909:41, pl. 1, fig.7; 1910:252; 1911:29. - Curran, 
1941:284. - Blackwelder, 1945:462. Distr.: México, Guatemala.

cinctus Herbst, 1799:372, pl. 137, fig. 11 (Erotylus). Tp. ? - (Suriname). Duponchel, 1825:171. - Crotch, 1876:544 (Zonarius). - Gemminger \& Harold, 1876:3716. - Kuhnt, 1909:42; 1910:253; 1911:29. - Curran, 1941:284. - Deelder, 1942:69. - Blackwelder, 1945:462. Distr.: Suriname. Guiana Francesa. Brasil, região amazônica.

discoideus Olivier, 1807:482, pl. 3, fig. 33 (Erotylus). - Duponchel, 1825:162, pl. 7, fig. 75. - Lacordaire, 1842:473 (syn.).

convexiusculus Crotch, 1876:544 (Zonarius). Tp.: MCZC exam. aut. - (Amazonas). - Gemminger \& Harold, 1876:3716. Kuhnt, 1909:42; 1910:253; 1911:30. - Curran, 1941:284. - Mader, 1942d:166; 1951:200. - Blackwelder, 1945:462. Distr.: Brasil, região amazônica.

var. sanguinicollis Kuhnt, 1910:251, 253. Tp. ? - (Brasil, Bahia). - Blackwelder, 1945:462. - Mader, 1951:200. Distr.: Brasil, região sudeste.

convexus Crotch, 1876:544 (Zonarius). Tp.: MCZC exam. aut. - (Amazonas). Gemminger \& Harold, 1876:3716. - Kuhnt, 1909:42; 1910:253; 1911:30. - Curran, 1941:284. - Blackwelder, 1945:462. Distr.: Brasil, região amazônica.

duodecimmaculatus Kuhnt, 1910:251, 252 (Zonarius). Tp.: ZMHU exam. aut. (Brasil); 1911:30. - Blackwelder, 1945:462. - Guérin, 1948:17. Distr.: Brasil, regiões sudeste e sul.

erythrogonus Crotch, 1876:543 (Zonarius). Tp.: MCZC exam. aut. -(Amazonas). - Gemminger \& Harold, 1876:3716. - Kuhnt, 1909:42; 1910:252; 1911:30.

- Curran, 1941:284. - Blackwelder, 1945:462. Distr.: Brasil, região amazônica.

fractus Crotch, 1876:544 (Zonarius). Tp.: MCZC exam. aut. - (Colômbia, Bogotá). - Gemminger \& Harold, 1876:3716. - Kuhnt, 1909:42; 1910:251, 253; 1911:30. - Curran, 1941:284. - Deelder, 1942:69. - Blackwelder, 1945:462. Distr.: Colômbia.

hybridus Erichson, 1847:177 (Erotylus). Tp.: ZMHU exam. aut. - (Peru). Crotch, 1876:543 (Zonarius). - Gemminger \& Harold, 1876:3716. - Kuhnt, 1909:42; 1910:252; 1911:30. - Curran, 1941:284. - Mader, 1942d:166; 1951:200. - Blackwelder, 1945:462. Distr.: Equador. Peru.

indicus Herbst, 1783:52, pl. 23, fig. 5 (Chrysomela). Tp.: ZMHU exam. aut. (Índia). - Gmelin, 1790:1685. - Olivier, 1792:435 (Erotylus); 1807:474, pl. 2, fig. 17. - Duponchel, 1825:175. - Lacordaire, 1842:470 (Zonarius). - Crotch, 1876:542. - Gemminger \& Harold, 1876:3716 - Kuhnt, 1909:41; 1910:251; 1911:30. - Curran, 1941:284. - Deelder, 1942:69. - Blackwelder, 1945:462. Distr.: Suriname. Guiana Francesa.

bifasciatus Herbst, 1799:370, pl. 137, fig. 9 (Erotylus). - Lacordaire, 1842:471 (syn.).

xanthomelas Lacordaire, 1842:469. - Crotch, 1876:542 (syn.). 
jansoni Crotch, 1873b:149 (Zonarius). Tp.: NHML exam. aut. - (Nicarágua, Chontales, Santo Domingo); 1876:543. - Gemminger \& Harold, 1876: 3716. - Gorham, 1888:109, pl. 6, figs 15, 16. - Kuhnt, 1909:42; 1910:253; 1911:30. - Deelder, 1942:69. - Blackwelder, 1945:462. Distr.: Nicarágua. Costa Rica. Panamá.

limbatus Kuhnt, 1910:249, 253, fig. 14 (Zonarius). Tp.: ZMHU exam. aut. (Colômbia); 1911:30. - Blackwelder, 1945:462. Distr.: Colômbia. Venezuela.

melanoderes Kuhnt, 1910:250, 252 (Zonarius). Tp.: ZMHU exam. aut. - (Juntas); 1911:30. - Schenkling, 1919:83. - Blackwelder, 1945:462. Distr.: Colômbia.

militarioides Mader, 1942d:166, 188 (Zonarius). Tp. ? - (Peru, Cuzco, Valle Assunción, Santa Isabel); 1951:200. Distr.: Peru.

militaris Germar, 1824:611 (Erotylus). Tp. ? - (Brasil). - Lacordaire, 1842:475 (Zonarius). - Crotch, 1876:542. - Gemminger \& Harold, 1876:3716. Kuhnt, 1909:42; 1910:252; 1911:30. - Bruch, 1914:384. - Curran, 1941: 284. - Deelder, 1942:69. - Blackwelder, 1945:462. - Guérin, 1948:17.

Distr.: Brasil, regiões sudeste e sul. Argentina.

decemmaculatus Duponchel, 1825:46, pl. 1, fig. 23. - Lacordaire, 1842:475 (syn.).

nigrotaeniatus Lacordaire, 1842:477 (Zonarius). Tp.: MCZC exam. aut. (Colômbia). - Crotch, 1876:543. - Gemminger \& Harold, 1876:3716. - Kuhnt, 1909:42; 1910:252; 1911:30. - Curran, 1941:284. - Blackwelder, 1945: 462. Distr.: Colômbia.

nigrotibialis Demay, 1838:24 (Erotylus). - Tp. ? - (Guiana Francesa) - Lacordaire, 1842:472 (Zonarius). - Crotch, 1876:542. - Gemminger \& Harold, 1876: 3716. - Kuhnt, 1909:41; 1910:251; 1911:30. - Curran, 1941:284. Blackwelder, 1945:462. Distr.: Bolívia. Guiana Francesa.

peregrinus Lacordaire, 1842:476 (Zonarius). Tp.: MNHN exam. aut. - (Brasil). - Crotch, 1876:542. - Gemminger \& Harold, 1876:3716. - Kuhnt, 1909:42; 1910:252; 1911:30. - Curran, 1941:284. - Deelder, 1942:69. - Blackwelder, 1945:462. - Guérin, 1948:18. Distr.: Brasil, região sudeste.

peruvianus Mader, 1942d:167, 189 (Zonarius). Tp.: MGFM exam. aut. - (Peru, Chanchamayo); 1951:200. Distr.: Peru.

rugipunctatus Crotch, 1876:544 (Zonarius). Tp.: MCZC exam. aut. - (Equador). - Gemminger \& Harold, 1876:3716. - Kuhnt, 1909:42; 1910:253; 1911:30.

- Curran, 1941:284. - Blackwelder, 1945:462. Distr.: Equador.

thoracicus Gistel, 1848:123. Tp. ? - [Localidade não citada]. Distr.: ?

trizonatus Germar, 1824:611 (Erotylus). Tp. ? - (Brasil). - Lacordaire, 1842:479 (Zonarius). - Crotch, 1876:542. - Gemminger \& Harold, 1876:3717. Kuhnt, 1909:42; 1910:252; 1911:30. - Curran, 1941:284. - Blackwelder, 1945:462. Distr.: Brasil. 
zebra Fabricius, 1787:92 (Erotylus). Tp.: ZMCD - (Caiena); 1792:38; 1801:6. Olivier, 1792:434. - Gmelin, 1790:1728. - Herbst, 1799:378. - Schönherr, 1808:327. - Latreille, 1811:167, pl. 16, fig. 10. - Duponchel, 1825:45, pl. 1, fig. 21. - Lacordaire, 1842:478 (Zonarius). - Crotch, 1876:543. Gemminger \& Harold, 1876:3717. - Gorham, 1888: 109. - Kuhnt, 1909:42; 1910:253; 1911:30. - Curran, 1941:284. - Deelder, 1942:69. - Blackwelder, 1945:462. - Pallister, 1955b:6 (Alloiotelus). Distr.: México. Belize. Guatemala. Trinidad. Colômbia. Equador. Peru. Guiana Francesa.

var. guatemalae Crotch, 1876:543. Tp.: MCZC exam. aut. - (Guatemala). Gemminger \& Harold, 1876:3716. - Kuhnt, 1909:42; 1910:253; 1911:

31. - Blackwelder, 1945:462. Distr.: Guatemala.

var. quadrifasciata Crotch, 1876:543. Tp.: MCZC exam. aut. - (México). Gemminger \& Harold, 1876:3716. - Gorham, 1909:42; 1910:253; 1911:31. - Blackwelder, 1945:462. Distr.: México.

\section{Scaphidomorphus Hope, 1841}

Scaphidomorphus Hope, 1841:111 (tgen. Chrysomela quinquepunctata Linnaeus, 1767:586, desig. orig.). - Lacordaire, 1842:32, 481. - Crotch, 1873b:149; 1876:545. - Chapuis, 1876:50, 66. - Gorham, 1888:110. - Kuhnt, 1909:10, 42. - Deelder, 1942:52. - Alvarenga, 1965:89.

bosci Guérin-Méneville, 1841a:117. Tp.: MCZC exam. aut. - (Colômbia). Lacordaire, 1842:482. - Crotch, 1876:545. - Gemminger \& Harold, 1876:3717. - Gorham, 1888:110, pl. 6, fig. 11. - Kuhnt, 1909:43; 1911:31. - Deelder, 1942:69. - Mader, 1942d:167; 1951:201. - Blackwelder, 1945: 462. - Guérin, 1948:18. Distr.: Do Panamá até o Brasil, região amazônica.

quinquepunctatus Linnaeus, 1767:586 (Chrysomela). Tp. ? - (América). - Fabricius, 1775:123 (Erotylus); 1781:157; 1787:91; 1792:37; 1801:5. - Gmelin, 1790:1727. - Olivier, 1792:433; 1807:470, pl. 1, fig. 5. - Illiger, 1806:231. - Herbst, 1799:357, pl. 136, fig. 7. - Schönherr, 1808:326. - Duponchel, 1825:41, pl. 1, fig. 12. - Sturm, 1826:139. - Lacordaire, 1842:483 (Scaphidomorphus). - Fauvel, 1860:326. - Crotch, 1876:545. - Kuhnt, 1909:43; 1911:31. - Bruch, 1914:381. - Curran, 1941:283. - Deelder, 1942:69. - Blackwelder, 1945:462. - Guérin, 1948:18. Distr.: Da Colômbia até a Argentina.

pentastictus Harold, 1875: 185. - Kuhnt, 1909:43 (syn.).

tripunctatus Guérin, 1946a:274, fig. 1. Tp.: CDSP exam. aut. - (Brasil, Pará, Santarém); 1948:18. Distr.: Brasil, região amazônica. 


\section{Prepopharus Erichson, 1847}

Prepopharus Erichson, 1847:177 (Tgen. Erotylus notatus Olivier, 1792:435, seg. Curran, 1941:283). - Chapuis, 1876:49, 67. - Gorham, 1888:110. - Kuhnt, 1909:10, 43. - Curran, 1941:283. - Deelder,1942:52. - Mader, 1942a:221. Alvarenga, 1965:88.

americanus Voet, 1778:44, pl. 33, fig. 6 (Pseudochrysomela). Tp. ? - [Localidade não citada]. - Alvarenga, 1977:105. Distr.: Guiana. Suriname. Guiana Francesa. Peru. Bolívia. Brasil, região amazonica.

notatus Olivier, 1792:435 (Erotylus). Tp. ? - (Caiena); 1807:471, pl. 1, fig. 11. - Fabricius, 1792:37; 1801:4. - Herbst, 1799:371, pl. 137, fig. 10. - Illiger, 1806:230. - Schönherr, 1808:326. - Duponchel, 1825:40, pl. 1, fig. 10. - Lacordaire, 1842:484 (Scaphidomorphus). - Crotch, 1876:545. - Gemminger \& Harold, 1876:3718 (Prepopharus). - Kuhnt, 1909:43, pl. 1, fig. 9; 1911:32. - Curran, 1941:283. - Deelder, 1942:69. - Mader, 1942d:167; 1951:201. - Blackwelder, 1945:463. - Guérin, 1948: 18. - Alvarenga, 1977:105 (syn.).

amoenus Mader, 1942a:220, 221. Tp.: MGFM exam. aut. - (Brasil, Santa Catarina, Mafra). Distr.: Brasil, região sul.

barytopoides Crotch, 1876:549 (Scaphidomorphus). Tp.: MCZC exam. aut. (Amazonas). - Gemminger \& Harold, 1876:3717 (Prepopharus). - Kuhnt, 1909:43; 1911:31. - Blackwelder, 1945:462. Distr.: Brasil, região amazônica.

bitaeniatus Lacordaire, 1842:486 (Scaphidomorphus). Tp.: MCZC exam. aut. (Brasil). - Kirsch, 1876:109. - Crotch, 1876:546. - Gemminger \& Harold, 1876:3717 (Prepopharus). - Kuhnt, 1909:43; 1911:31. - Mader, 1942d: 167; 1951:201. - Blackwelder, 1945:462. - Delkeskamp, 1957:97. Distr.:

Peru. Bolívia. Brasil, região central.

spectabilis Deelder, 1942:66, fig. 1g (Erotylus). - Delkeskamp, 1957:103 (=Prepopharus bitaeniatus).

chamoeleo Lacordaire, 1842:489 (Scaphidomorphus). Tp.: MNHN exam. aut. (Colômbia). - Crotch, 1876:548. - Gemminger \& Harold, 1876:3717 (Prepopharus). - Kunht, 1909:44; 1911:31. - Blackwelder, 1945:462. Distr.: Colômbia. Bolívia.

diffinis Erichson, 1847:178. Tp. ? - (Peru). - Crotch, 1867:549 (Scaphidomorphus). - Gemminger \& Harold, 1876:3717 (Prepopharus). - Kunht, 1909: 44; 1911:31. - Mader, 1942a:221; 1942d:167; 1951:202. - Blackwelder, 1945:462. Distr.: Peru.

disputabilis Crotch, 1876:548 (Scaphidomorphus). Tp.: MCZC exam. aut. (Amazonas). - Gemminger \& Harold, 1876:3718 (Prepopharus). - Kuhnt, 1909:43; 1911:31. - Mader,1942a:221. - Blackwelder, 1945:462. Distr.: Brasil, região amazônica. 
duplicatus Crotch, 1876:549 (Scaphidomorphus). Tp.: MCZC exam. aut. (Amazonas). - Gemminger \& Harold, 1876:3718 (Prepopharus). - Kunht, 1909:44; 1911:31. - Mader, 1942a:221. - Blackwelder, 1945:462. Distr.: Brasil, região amazônica.

duponcheli Chevrolat, 1834, fasc.4 (Erotylus). Tp.: MNHN exam. aut. - (México, Orizaba, Alvarado). - Lacordaire, 1842:48 (Scaphidomorphus). - Crotch, 1876:546. - Gemminger \& Harold, 1876:3718 (Prepopharus). - Gorham, 1888:111, pl. 6, figs 17, 18. - Kuhnt, 1909:44; 1911:31. - Blackwelder, 1945:462. Distr.: México. América Central.

eduardoi Alvarenga, 1976:512. Distr.: Colômbia. Venezuela. Guiana. Guiana Francesa. Peru. Brasil, região amazônica.

undatus Fabricius, 1801 nec Olivier, 1792. - Fabricius, 1801:8 (Erotylus). Tp.: ZMCD - (América meridional). - Olivier, 1807:475, pl. 2, fig. 21. - Schönherr, 1808:328. - Duponchel, 1825:171. - Zimsen, 1964: 115. - Lacordaire, 1842:491 (Scaphidomorphus). - Crotch, 1876:546. - Gemminger \& Harold, 1876:3718 (Prepopharus). - Kunht, 1909:44; 1911:32. - Mader, 1942d:167; 1951:202. - Blackwelder, 1945:463. Guérin, 1948:18. - Alvarenga, 1976:512 (nom.praeoc.).

var. praestus Duponchel, 1825:55, pl. 2, fig. 45 (Erotylus). Tp. ? (Cayenne). - Lacordaire, 1842:490 (Scaphidomorphus). - Crotch, 1876:547. Gemminger \& Harold, 1876:3718 (Prepopharus). - Kuhnt, 1909:44; 1911:32. - Blackwelder, 1945:463. Distr.: Guiana Francesa.

var. zigaena Lacordaire, 1842:492 (Scaphidomorphus). Tp. ? - (Colômbia). Crotch, 1876:547. - Gemminger \& Harold, 1876:3718 (Prepopharus). - Kunht, 1909:44; 1911:32. - Blackwelder, 1945:463. Distr.: Colômbia.

var. atroflavus Crotch, 1876:547 (Scaphidomorphus). Tp.: MCZC exam. aut. - [Localidade não citada]. - Gemminger \& Harold, 1876:3718 (Prepopharus). - Kunht, 1909:44; 1911:32. - Mader, 1942d:167; 1951:202. Blackwelder, 1945:463. Distr.: Guiana Francesa. Peru.

var. interruptus Crotch, 1876:547 (Scaphidomorphus). Tp.: MCZC exam. aut. - [Localidade não citada]. - Gemminger \& Harold, 1876:3718 (Prepopharus). - Kunht, 1909:44; 1911:32. - Blackwelder, 1945:463. Distr.: Guiana Francesa.

var. deficiens Crotch, 1876:547 (Scaphidomorphus). Tp.: MCZC exam. aut. - [Localidade não citada]. - Gemminger \& Harold, 1876:3718 (Prepopharus). - Kunht, 1909:44; 1911:32. - Blackwelder, 1945:463. Distr.: Guiana Francesa.

var. thoracicus Crotch, 1876:547 (Scaphidomorphus). Tp. MCZC exam. aut. - [Localidade não citada]. - Gemminger \& Harold, 1876:3718 (Prepopharus). - Kunht, 1909:44; 1911:32. - Blackwelder, 1945:463. Distr.: Guiana Francesa.

var. inchoatus Crotch, 1876:547 (Scaphidomorphus). Tp.: MZCZ exam. aut. 
- [Localidade não citada]. - Gemminger \& Harold, 1876:3718 (Prepopharus). - Kunht, 1909:44; 1911:32. - Blackwelder, 1945:463. Distr.: Guiana Francesa.

var. basisignatus Crotch, 1876:547 (Scaphidomorphus). Tp.: MCZC exam. aut. - [Localidade não citada]. - Gemminger \& Harold, 1876:3718 (Prepopharus). - Kunht, 1909:44; 1911:32. - Blackwelder, 1945:463. Distr.: Venezuela.

var. polyscriptus Kuhnt, 1910:270, fig. 26. Tp. ? - (Caiena); 1911:32. Blackwelder, 1945:463. Distr.: Guiana Francesa.

herbsti Lacordaire, 1842:486 (Scaphidomorphus). Tp. ? - (Bolívia, Chiquitos). Crotch, 1876:546. - Gemminger \& Harold, 1876:3718 (Prepopharus). Kuhnt, 1909:43; 1911:32. - Bruch, 1914:381. - Deelder, 1942: 69. Mader, 1942d:167; 1951:201. - Blackwelder, 1945:462. - Guérin, 1948: 18. Distr.: Peru. Bolívia. Brasil. Argentina.

var. crabronoides Lacordaire, 1842:487 (Scaphidomorphus). Tp.: MCZC exam. aut. - (Pará). - Crotch, 1876:546. - Gemminger \& Harold, 1876:3718 (Prepopharus). - Kuhnt, 1909:43; 1911:32. - Mader, 1942d: 167; 1951:201. - Blackwelder, 1945:462. - Guérin, 1948:18. Distr.: Brasil, região amazônica.

impluviatus Lacordaire, 1842:485 (Scaphidomorphus). Tp. ? - (Colômbia).

Kirsch, 1876:109. - Crotch, 1876:545. - Gemminger \& Harold, 1876:3718 (Prepopharus). - Kuhnt, 1909:43; 1911:32. - Deelder, 1942:69. - Mader, 1942d:167; 1951:201. - Blackwelder, 1945:462. Distr.: Panamá. Colômbia. Equador. Peru.

maculiforus Crotch, 1876:548 (Scaphidomorphus). Tp.: MCZC exam. aut. (Amazonas). - Gemminger \& Harold, 1876:3718 (Prepopharus). -Kunht, 1909:44: 1911:32. - Mader, 1942a:221. - Blackwelder, 1945:462. Distr.: Brasil, região amazônica.

obliteratus Erichson, 1847:178. Tp.: ZMHU exam. aut. - (Peru). - Crotch, 1876:549 (Scaphidomorphus). - Gemminer \& Harold, 1876:3718 (Prepopharus). - Kunht, 1909:44; 1911:32. - Mader, 1942d:167; 1951:202. Blackwelder, 1945:463. Distr.: Peru.

obscurior Curran, 1941:283, pl. 1, fig. 4. Tp.: DTNY - (Guiana, Kartabo). Distr.: Guiana.

opalizans Lacordaire, 1842:493 (Scaphidomorphus). Tp. ? - (Caiena). - Crotch, 1876:548. - Gemminger \& Harold, 1876:3718 (Prepopharus). - Kunht, 1909:44; 1911:32. - Curran, 1941:283. - Mader, 1942a:221. - Blackwelder, 1945:463. Distr.: Guiana Francesa.

partitus Crotch, 1876:548 (Scaphidomorphus). Tp.: MCZC exam. aut. - (Equador). - Gemminger \& Harold, 1876:3718 (Prepopharus). - Kuhnt, 1909:44; 1911:32. - Mader, 1942d:167; 1951:202. - Blackwelder, 1945:463. Distr.: Colômbia. Equador. Peru. 
pustuliforus Crotch, 1876:548 (Scaphidomorphus). Tp.: MCZC exam. aut. (Amazonas). - Gemminger \& Harold, 1876:3718 (Prepopharus). - Kunht, 1909:44; 1911:32. - Mader, 1942a:221. - Blackwelder, 1945:463. Distr.: Brasil, região amazônica.

tricolor Mader, 1942d:167, 190. Tp.: DEIB - (Peru, Chanchamayo); 1951:202. Distr.: Peru.

varicollis Crotch, 1876:549 (Scaphidomorphus). Tp.: MCZC exam. aut. - (Amazonas). - Gemminger \& Harold, 1876:3718 (Prepopharus). - Kuhnt, 1909:44; 1911:32. - Blackwelder, 1945:463. Distr.: Brasil, região amazônica.

xanthomelas apicenigrum Mader, 1942c:570. Tp.: MGFM exam. aut. - (Colômbia, rio Dágua). Distr.: Colômbia.

xanthomelas xanthomelas Crotch, 1873b:150 (Scaphidomorphus). Tp.: MCZC exam. aut. - (Nicarágua, Chontales, Santo Domingo); 1876:546. - Gemminger \& Harold, 1876:3718 (Prepopharus). - Gorham, 1888:111, pl. 6, fig. 12. - Kunht, 1909:44; 1911:32. - Blackwelder, 1945:463. Distr.: Nicarágua. Costa Rica.

\section{Perithonius Crotch, 1876.}

Perithonius Crotch, 1876:549 (Tgen. Euphanistes misolampoides Lacordaire, 1842:257, desig. orig. ). - Kunht, 1909:9, 44. - Deelder, 1942:51. - Alvarenga, 1965:88.

misolampoides Lacordaire, 1842:257 (Euphanistes). Tp. ? -(Colômbia). - Crotch, 1876:549 (Perithonius). - Gemminger \& Harold, 1876:3703. - Kunht, 1909:44; 1911:32. - Blackwelder, 1945:463. Distr.: Colômbia.

Rhynchothonius Crotch, 1876.

Rhynchothonius Crotch, 1876:550 (Tgen. Rhynchothonius albidoguttatus Crotch, 1876:550, monotipia). - Kuhnt, 1909:8, 45. - Deelder, 1942:51. - Alvarenga, 1965:89.

albidoguttatus Crotch, 1876:550. Tp.: MCZC exam. aut. - (Amazonas). - Gemminger \& Harold, 1876:3718. - Kuhnt, 1909:45; 1911:33. - Blackwelder, 1945:463. Distr.: Brasil, região amazônica. 


\section{Neopriotelus Alvarenga, 1965.}

Neopriotelus Alvarenga, 1965:87 (Tgen. Erotylus octomaculatus Olivier, 1792: 436, desig. orig.).

Priotelus Hope, 1841. - Hope, 1841:112 (Tgen. Erotylus serripennis GúerinMéneville, 1841:119, desig. orig.). - Lacordaire, 1842:32, 493. - Chapuis, 1876:50, 70. - Crotch, 1876: 550. - Gorham, 1888:111. - Kuhnt, 1909:10, 45. - Deelder, 1942:52. - Alvarenga, 1965:88 (nom. praeoc., Gray, 1840).

apiatus Chevrolat, 1835, fasc. 5 (Erotylus). Tp.: MCZC exam. aut. - (México, Vera Cruz, Orizaba). - Lacordaire, 1842:498 (Priotelus). - Crotch, 1876: 552. - Gemminger \& Harold, 1876:3718. - Gorham, 1888:112, pl. 6, fig. 20. - Kuhnt, 1909:46; 1911:33. - Deelder, 1942:69. - Blackwelder, 1945:463. - Guérin, 1948:18. Distr.: Do México até a Colômbia.

bimaculatus Mader, 1942d:168, 192 (Priotelus). Tp.: MGFM exam. aut. - (Peru, Oxapampa); 1951:203. Distr.: Peru.

calceatus Lacordaire, 1842:496 (Priotelus). Tp. ? - (Caiena). - Crotch, 1876:553.

- Gemminger \& Harold, 1876:3719. - Kuhnt, 1909:46; 1911:33. - Blackwelder, 1945:463. Distr.: Guiana Francesa.

debilis Crotch, 1876:552 (Priotelus). Tp.: MCZC exam. aut. - (Amazonas). Gemminger \& Harold, 1876:3719. - Kuhnt, 1909:46; 1911:33. - Blackwelder, 1945:463. Distr.: Brasil, região amazônica.

dejeani Lacordaire, 1842:501 (Priotelus). Tp. ? - (Caiena). - Crotch, 1876:550.

- Gemminger \& Harold, 1876:3719. - Kuhnt, 1909:46; 1911:33. - Blackwelder, 1945:463. Distr.: Guiana Francesa.

detrahens Crotch, 1876:551 (Priotelus). Tp.: MCZC exam. aut. - (Amazonas). Gemminger \& Harold, 1876:3719. - Kuhnt, 1909:46; 1911:33. - Blackwelder, 1945:463. - Delkeskamp, 1957:105. Distr.: Equador. Brasil, região amazônica.

difficilis Crotch, 1876:553 (Priotelus). Tp.: MCZC exam. aut. - (Colômbia). Gemminger \& Harold, 1876:3719. - Kuhnt, 1909:46; 1911:33. - Blackwelder, 1945:463. Distr.: Colômbia.

elegans Mader, 1942d:167, 191 (Priotelus). Tp.: MGFM exam. aut. - (Peru, Oxapampa); 1951:203. Distr.: Peru.

equestris Lacordaire, 1842:495 (Priotelus). Tp. ? - (Caiena). - Crotch, 1876:553.

- Gemminger \& Harold, 1876: 3719. - Kuhnt, 1909:46; 1911:33. Blackwelder, 1945:463. Distr.: Guiana Francesa. Brasil, região amazônica.

var. amazonicus Crotch, 1876:553. Tp.: MCZC exam. aut. - (Amazonas). Kuhnt, 1909:46; 1911:33. - Blackwelder, 1945:463. Distr.: Brasil, região amazônica. 
femoralis Kirsch, 1865:102 (Bacis). Tp.: SMTD exam. aut. - (Colombia, Bogotá). - Crotch, 1876:553 (Priotelus). - Gemminger \& Harold, 1876:3719. Kuhnt, 1909:46; 1911:33. - Blackwelder, 1945:463. Distr.: Colômbia. Venezuela.

habrodactyloides Crotch, 1876:554 (Priotelus). Tp.: MCZC exam. aut. - (Amazonas). - Gemminger \& Harold, 1876:3719. - Kuhnt, 1909:46; 1911:33. Blackwelder, 1945:463. Distr.: Brasil, região amazônica.

horioni Delkeskamp, 1957:103, fig. 5 (Priotelus). Tp.: ZMHU ex aut. - (Bolívia, Chapare, $400 \mathrm{~m})$. Distr.: Bolívia.

ignobilis Kirsch, 1865:102 (Priotelus). Tp.: SMTD exam. aut. - (Colômbia, Bogotá). - Crotch, 1876:552. - Gemminger \& Harold, 1876:3719. - Kuhnt, 1909:46; 1911:33. - Blackwelder, 1945:463. Distr.: Colômbia.

irroratus Lacordaire, 1842:331 (Brachysphoenus). Tp. ? - (Caiena). - Crotch, 1876:554 (Priotelus). - Gemminger \& Harold, 1876:3719. - Kuhnt, 1909: 46; 1911:33. - Blackwelder, 1945:463. Distr.: Guiana Francesa.

limbatus Crotch, 1876:553 (Priotelus). Tp.: MCZC exam. aut. - (Equador, Guayaquil). - Gemminger \& Harold, 1876:3719. - Kuhnt, 1909:46; 1911:33. Blackwelder, 1945:463. Distr.: Equador.

lineatulus Crotch, 1876:555 (Priotelus). Tp.: MCZC exam. aut. - (Amazonas). Gemminger \& Harold, 1876:3719. - Kuhnt, 1909:46; 1911:33. - Blackwelder, 1945:463. Distr.: Brasil, região amazônica.

lividus Lacordaire, 1842:496 (Priotelus). Tp. ? - (Brasil). - Crotch, 1876:553. Gemminger \& Harold, 1876:3719. - Kuhnt, 1909:46; 1911:33. - Blackwelder, 1945:463. - Guérin, 1948:18. Distr.: Brasil, região sudeste.

macasensis Crotch, 1876:551 (Priotelus). Tp.: MCZC exam. aut. - (Equador).

Gemminger \& Harold, 1876:3719. - Kuhnt, 1909:46; 1911:33. - Blackwelder, 1945:463. - Delkeskamp, 1957:105. Distr.: Equador.

minor Crotch, 1876:554 (Priotelus). Tp.: MCZC exam. aut. - (Amazonas). Gemminger \& Harold, 1876:3719. - Kuhnt, 1909:46; 1911:33. - Blackwelder, 1945:463. Distr.: Brasil, região amazônica.

nitidior Crotch, 1876:554 (Priotelus). Tp.: MCZC exam. aut. - (Amazonas). Gemminger \& Harold, 1876:3719. - Kuhnt, 1909:46; 1911:33. - Blackwelder, 1945:463. Distr.: Brasil, região amazônica.

obsoletus Crotch, 1876:552 (Priotelus). Tp.: MCZC exam. aut. - (Brasil). Gemminger \& Harold, 1876:3719. - Kuhnt, 1909:46; 1911:33. - Blackwelder, 1945:463. - Guérin, 1948:18. Distr.: Brasil, região amazônica.

octomaculatus Olivier, 1792:436 (Erotylus). Tp. ? - (Suriname); 1807:476, pl. 2, fig. 22. - Fabricius, 1801:5. - Schönherr, 1808:327. - Duponchel, 1825: 170. - Lacordaire, 1842:500 (Priotelus). - Crotch, 1876:550. - Gemminger \& Harold, 1876:3719. - Kuhnt, 1909:45; 1911:33. - Deelder, 1942:69. Blackwelder, 1945:463. Distr.: Guiana Francesa. Suriname.

serripennis Guérin-Méneville, 1841a:119. - Lacordaire, 1842:500 (syn.). 
orphanus Crotch, 1876:551 (Priotelus). Tp.: MCZC exam. aut. - (Amazonas). Gemminger \& Harold, 1876:3719. - Kuhnt, 1909:46; 1911:33. - Blackwelder, 1945:463. Distr.: Brasil, região amaz\%nica.

ovatus Crotch, 1876:554 (Priotelus). Tp.: MCZC exam. aut. - (Amazonas). Gemminger \& Harold, 1876:3719. - Kuhnt, 1909:46; 1911:33. - Blackwelder, 1945:463. Distr.: Brasil, região amazônica.

peruvianus Mader, 1942d:168, 192 (Priotelus). Tp. ? - (Peru, Sivia, 520 m); 1951:203. - Delkskamp, 1957:97, 104, 105. Distr.: Peru. Bolívia.

quadrimaculatus Guérin, 1956:60, fig. 23 (Priotelus). Tp.: CARJ exam. aut. (Brasil, Pará, Mocajuba, Mangabeira). Distr.: Brasil, região amazônica. rugithorax Crotch, 1876:555 (Priotelus). Tp.: MCZC exam. aut. - (Amazonas). - Gemminger \& Harold, 1876:3719. - Kuhnt, 1909:46; 1911:33. - Blackwelder, 1945:463. Distr.: Brasil, região amazônica.

sexmaculatus Crotch, 1876:551 (Priotelus). Tp.: MCZC exam. aut. - (Amazonas). - Gemminger \& Harold, 1876:3719. - Kuhnt, 1909:46; 1911:33. - Blackwelder, 1945:463. Distr.: Brasil, região amazônica.

spinolae Guérin-Méneville, 1844:312 (Omoitelus). Tp.: MNHN exam. aut. (Colômbia). - Crotch, 1876:553 (Priotelus). - Gemminger \& Harold, 1876:3719. - Kuhnt, 1909:46; 1911:33. - Blackwelder, 1945:463. Distr.: Colômbia.

stellio Erichson, 1847:177 (Priotelus). Tp.: ZMHU exam. aut. - (Peru). - Crotch, 1876:552. - Gemminger \& Harold, 1876:3719. - Kuhnt, 1909:46; 1911:33. - Mader, 1942d:168; 1951:203. - Blackwelder, 1945:463. Distr.: Peru.

tigrinipennis Lacordaire, 1842:499 (Priotelus). Tp. ? - (Colômbia). - Crotch, 1876:551. - Gemminger \& Harold, 1876:3719. - Kuhnt, 1909:46; 1911:33.

- Deelder, 1942:70. - Blackwelder, 1945:463. - Delkeskamp, 1957:104. Distr.: Colômbia. Venezuela.

transversofasciatus Crotch, 1876:551 (Priotelus). Tp.: MCZC exam. aut.

(Colômbia). - Kuhnt, 1909:46; 1911:34. - Blackwelder, 1945:463.

Gemminger \& Harold, 1876:3719 (Bacis). Distr.: Colômbia.

tricolor Fabricius, 1801:8 (Erotylus). Tp.: ZMCD - (América meridional).

Schönherr, 1808:328. - Dupochel, 1825:172. - Lacordaire, 1842:517. -

Gemminger \& Harold, 1876:3715. - Zimsen, 1964:115. - Chevrolat, 1843:80 (Priotelus). Distr.: Guiana Francesa.

jucundus Lacordaire, 1842:497 (Priotelus). Tp. ? - (Caiena). - Crotch, 1876: 553. - Gemminger \& Harold, 1876:3719. - Kuhnt, 1909:46; 1911:33. - Blackwelder, 1945:463. - Chevrolat, 1843:80 (syn.).

truncatus Lacordaire, 1842:499 (Priotelus). Tp.: MCZC exam. aut. - (Caiena). Crotch, 1876:552. - Gemminger \& Harold, 1876:3719. - Kuhnt, 1909:46; 1911:34. - Blackwelder, 1945:463. Distr.: Guiana Francesa.

zebra Kuhnt, 1910:269, fig. 24 (Priotelus). Tp.: ZMHU exam. aut. - (Peru, rio Manes); 1911:34. - Mader, 1942d:167; 1951:202. - Blackwelder, 1945: 
463. Distr.: Peru.

\section{Tapinotarsus Kirsch, 1865}

Tapinotarsus Kirsch, 1865:100 (Tgen. Tapinotarsus maculatus Kirsch, 1865:101, monotipia). - Chapuis, 1876:50, 69. - Crotch, 1876:555. - Kuhnt, 1909:10, 46. - Deelder, 1942:52. - Alvarenga, 1965:90.

brachymeroides Crotch, 1876:556. Tp.: MCZC exam. aut. - (Amazonas). Gemminger \& Harold, 1876:3717. - Kuhnt, 1909:47; 1911:34. - Blackwelder, 1945:463. Distr.: Brasil, região amazônica.

kirschi Crotch, 1876:556. Tp.: MCZC exam. aut. - (Amazonas). - Gemminger \& Harold, 1876:3717. - Kuhnt, 1909:47; 1911:34. - Blackwelder, 1945:463.

Distr.: Brasil, região amazônica.

maculatoides Mader, 1943:114. Tp.: SMTD exam. aut. - (Equador, Sabanilla). Distr.: Equador.

maculatus Kirsch, 1865:101. Tp.: SMTD exam. aut. - (Colômbia, Bogotá). Crotch, 1876:555. - Gemminger \& Harold, 1876:3717. - Kuhnt, 1909:47; 1911:34. - Blackwelder, 1945:463. Distr.: Colômbia. Brasil, região amazônica.

var. octomaculatus Kuhnt, 1910:270. Tp. ? - (Colômbia, Bogotá); 1911:34. Blackwelder, 1945:463. Distr.: Colômbia.

multinotatus Lacordaire, 1842:330 (Brachysphoenus). Tp. ? - (Caiena). - Crotch, 1876:555 (Tapinotarsus). - Gemminger \& Harold, 1876:3717. - Kuhnt, 1909:47; 1911:34. - Blackwelder, 1945:463. Distr.: Guiana Francesa. Brasil, região amazônica.

\section{Bacis Dejean, 1837}

Bacis Dejean, 1837:427 (Tgen. Erotylus tripunctatus Duponchel, 1825:160, seg. Crotch, 1876:556). - Hope, 1841:113. - Lacordaire, 1842:32, 502. - Chapuis, 1876:50, 68. - Crotch, 1876:556. - Kuhnt, 1909:10, 47. - Deelder, 1942:52. - Alvarenga, 1965:82.

immaculicollis Crotch, 1876:556. Tp.: MCZC exam. aut. - (Amazonas). Fleutiaux, 1886:224. - Kuhnt, 1909:48; 1911:34. - Blackwelder, 1945:463. Distr.: Brasil, região amazônica.

nigropictus Crotch, 1876:557. Tp.: MCZC exam. aut. - (Venezuela). - Fleutiaux, 1886:224. - Kuhnt, 1909:48; 1911:34. - Blackweder, 1945:463. Distr.: Venezuela.

scutellaris Lacordaire, 1842:504. Tp. ? - (Caiena). - Crotch, 1876:556. - Gemminger \& Harold, 1876:3719. - Kuhnt, 1909:48; 1911:34. - Blackwelder, 
1945:463. Distr.: Guiana Francesa.

var. ambiguus Lacordaire, 1842:505. Tp. ? - (Caiena). - Crotch, 1876:556. Gemminger \& Harold, 1876:3719. - Kuhnt, 1909:48; 1911:34. Blackwelder, 1945:463. Distr.: Guiana Francesa.

tripunctatus Duponchel, 1825:160, pl. 7, fig. 71 (Erotylus). Tp. ? - (Caiena). Lacordaire, 1842:503 (Bacis). - Crotch, 1876:556. - Gemminger \& Harold, 1876:3719. - Kuhnt, 1909:48; 1911:34. - Blackwelder, 1945:463. Distr.: Guiana Francesa.

\section{Phricobacis Crotch, 1876.}

Crotch, 1876:557 (Tgen. Phricobacis batesi Crotch, 1876:558, desig. orig.). Kuhnt, 1909:10, 48. - Deelder, 1942:52. - Alvarenga, 1965:88.

arduus Erichson, 1847:177 (Bacis). Tp.: ZMHU exam. aut. - (Peru). - Crotch, 1876:557 (Phricobacis). - Gemminger \& Harold, 1876:3719. - Kuhnt, 1909:48; 1911:34. - Curran, 1941:286. - Mader, 1942d:168; 1951:204. Blackwelder, 1945: 463. - Delkeskamp, 1957:97. Distr.: Peru. Bolívia. Brasil, região amazônica.

var. hepaticus Kirsch, 1876:110. Tp.: SMTD exam. aut. - (Peru, Pozuzo). Crotch, 1876:557. - Gemminger \& Harold, 1876:3719. - Kuhnt, 1909:48; 1911:34. - Curran, 1941:286. - Mader, 1942d:168; 1951:204. - Blackwelder, 1945:463. Distr.: Peru.

batesi Crotch, 1876:558. Tp.: MCZC exam. aut. - (Amazonas). - Gemminger \& Harold, 1876:3720. - Kuhnt, 1909:48; 1911:34. - Curran, 1941:286. Blackwelder, 1945:463. Distr.: Brasil, região amazônica.

beebei Curran, 1941:286, pl. 1, fig. 8. Tp.: DTNY (Guiana, Kartabo). Distr.: Guiana.

flavomaculatus Mader, 1943:114. Tp.: MGFM exam. aut. - (Peru, Chanchamayo); 1951:203. Distr.: Peru.

hopei Guérin-Méneville, 1841a:115 (Erotylus). Tp.: MNHN exam. aut. - (Bolívia). - Lacordaire, 1842:448. - Crotch, 1876:557 (Phricobacis). - Gemminger \& Harold, 1876:3720. - Kuhnt, 1909:48; 1911:34. - Curran, 1941:286. - Blackwelder, 1945:463. Distr.: Bolívia.

marginatus Guérin-Méneville, $1841 \mathrm{a}: 119$ (Omoiotelus). Tp.: MNHN exam. aut. - (Brasil). - Lacordaire, 1842:505 (Bacis). - Erichson, 1847:177. - Crotch, 1876:558 (Phricobacis). - Gemminger \& Harold, 1876:3720. - Kuhnt, 1909:48; 1911:34. - Curran, 1941:286. - Mader, 1942d:168; 1951:203. Blackwelder, 1945:463. Distr.: Peru. Bolívia. Brasil, região amazônica.

navicularis Lacordaire, 1842:512 (Omoiotelus). Tp.: MNHN exam. aut. - (Caiena). - Crotch, 1876:559 (Phricobacis). - Gemminger \& Harold, 1876:3720. - Kuhnt, 1909:48; 1911:34. - Curran, 1941:286. - Blackwelder, 1945:463. 
Distr.: Guiana Francesa. Brasil, região amazônica.

pulcher Crotch, 1876:558. Tp.: MCZC exam. aut. - (Amazonas). - Gemminger \& Harold, 1876:3720. - Kuhnt, 1909:48; 1911:34. - Curran, 1941:286. Blackwelder, 1945:463. Distr.: Brasil, região amazônica.

ratzeburgi Lacordaire, 1842:449 (Erotylus). Tp. ? - (Brasil). - Crotch, 1876:558 (Phricobacis). - Gemminger \& Harold, 1876:3720. - Kuhnt, 1909:48; 1911:35. - Curran, 1941:286. - Blackwelder, 1945:463. Distr.: Brasil.

rufolimbatus Crotch, 1876:558. Tp.: MCZC exam. aut. - (Amazonas). - Gemminger \& Harold, 1876:3720. - Kuhnt, 1909:48; 1911:35. - Curran, 1941:286. - Blackwelder, 1945:463. Distr.: Brasil, região amazônica.

\section{Ellipticus Chevrolat, 1837.}

Ellipticus Chevrolat, 1837:426 (Tgen. Erotylus testaceus Fabricius, 1775:822, seg. Alvarenga, 1965:83). - Alvarenga, 1965:83.

Omoiotelus Hope, 1841a:112 (Tgen. Erotylus testaceus Fabricius, 1775:822, desig. orig.). - Lacordaire, 1842:32, 506. - Chapuis, 1876:50, 71. - Curran, 1941:281. - Alvarenga, 1965:87 (syn.).

Homoeotelus Erichson, 1847:177. - Crotch, 1873b:150; 1876:559. - Gemminger \& Harold, 1876:3720. - Gorham, 1888:112. - Kuhnt, 1909:9, 49; 1911:35. Deelder, 1942:51. - Mader, 1942d:168. - Blackwelder, 1945:463. - Alvarenga, 1965:85 (syn.).

\section{Ellipticus (Ellipticus) Chevrolat, 1837.}

Ellipticus Chevrolat, 1837:426 (Tsubgen. Erotylus testaceus Fabricius, 1775:822, seg. Alvarenga, 1965;83). - Alvarenga, 1965:83.

Homoeotelus Mader, 1942d:168. - Alvarenga, 1965:85 (syn.).

albidipennis Crotch, 1876:562 (Homoeotelus). Tp.: MCZC exam. aut. - (Equador). - Gemminger \& Harold, 1876:3720. - Kuhnt, 1909:50; 1911:35. Blackwelder, 1945:463. Distr.: Equador.

apicicornis Guérin-Méneville, 1844:312 (Omoiotelus). Tp.: MNHN exam. aut. (Colômbia). - Crotch, 1876:560 (Homoeotelus). - Gemminger \& Harold, 1876:3720. - Kuhnt, 1909:50; 1911:35. - Blackwelder, 1945:463. Distr.:

Colômbia. Brasil, região amazônica.

emarginatus Kirsch, 1865:103 (Omoiotelus). - Crotch, 1876:560 (syn.).

atratus Fabricius, 1801:8 (Erotylus). Tp.: ZMCD - (América meridional). Schönherr, 1808:328. - Duponchel, 1825:174. - Lacordaire, 1842:516. Zimsen, 1964:115. -Chevrolat, 1843:80 (Omoiotelus). - Gemminger \& Harold, 1876:3720 (Homoeotelus). Distr.: ?

atripennis Mader, 1942d:169, 193 (Homoeotelus). Tp.: MGFM exam. aut. - 
(Peru, Pachitea); 1951:204. Distr.: Peru.

confusus Crotch, 1873b: 150 (Homoeotelus). Tp.: MCZC exam. aut. - (Nicarágua, Chontales, Santo Domingo); 1876:559. - Gemminger \& Harold, 1876: 3720. - Gorham, 1889:113, pl. 6, fig. 21. - Kuhnt, 1909:50; 1911:35. Deelder, 1942:70. - Blackwelder, 1945:463. Distr.: América Central.

d'orbignyi Guérin-Méneville, 1841:119 (Omoiotelus). Tp.: MCZC exam. aut. (Bolívia); 1844:311. - Crotch, 1876:559 (Homoeotelus). - Gemminger \& Harold, 1876:3720. - Kuhnt, 1909:50, pl. 1, figs 10, 10a; 1911:35. - Bruch, 1914:381. - Deelder, 1942:70. - Mader, 1942d:169; 1951:205. - Blackwelder, 1945:463. - Delkeskamp, 1957:98. Distr.: Colômbia. Peru. Bolívia. Brasil, região amazônica. Argentina.

distinguendus Crotch, 1876:560 (Homoeotelus). Tp.: MCZC exam. aut. - (Amazonas). - Gemminger \& Harold, 1876:3720. - Kuhnt, 1909:50; 1911:35. Blackwelder, 1945:463. Distr.: Brasil, região amazônica.

duponcheli Lacordaire, 1842:507 (Omoiotelus). Tp. ? - (Brasil). - Crotch, 1876: 559 (Homoeotelus). - Gemminger \& Harold, 1876:3720. - Kuhnt, 1909:50; 1911:35. - Blackwelder, 1945:463. Distr.: Brasil, região sudeste.

hepaticus Crotch, 1876:560 (Homoeotelus). Tp. ? - (Equador). - Gemminger \& Harold, 1876:3720. - Kuhnt, 1909:50; 1911:35. - Mader, 1942d:169; 1951:204. - Blackwelder, 1945:463. Distr.: Equador. Peru. Bolívia.

niger Deelder, 1942:70, fig. Ih (Homoeotelus). Tp.: RNHL - (Peru, Chanchamayo). Distr.: Peru.

octomaculatus Crotch, 1876:560 (Homoeotelus). Tp. ? - (Peru, Pozuzu). - Kirsch, 1876:111 (Omoiotelus). - Gemminger \& Harold, 1876:3720 (Homoeotelus). - Kuhnt, 1909:49; 1911:35. - Mader, 1942d:168; 1951:204. Blackwelder, 1945:463. Distr.: Peru.

orbignyanus Lacordaire, 1842:510 (Omoiotelus). Tp. ? - (Bolívia, Santa Cruz de la Sierra). - Crotch, 1876:559 (Homoeotelus). - Gemminger \& Harold, 1876:3720. - Kuhnt, 1909:50; 1911:35. - Deelder, 1942:70. - Mader, 1942d:169; 1951:205. - Blackwelder, 1945:463. - Guérin, 1948:18. Delkeskamp, 1957:98. Distr.: Peru. Bolívia. Brasil, região amazônica.

pallidus Olivier, 1792:436 (Erotylus). Tp. ? - (Suriname); 1807:478, pl. 2, fig. 26. - Duponchel, 1825:171. - Lacordaire, 1842:511 (Omoiotelus). - Curran, 1941:282. - Crotch, 1876:560 (Homoeotelus). - Gemminger \& Harold, 1876:3720. - Kuhnt, 1909:49; 1911:35. - Deelder, 1942:70. - Blackwelder, 1945:463. Distr.: Colômbia. Guiana. Suriname. Guiana Francesa. Brasil, região amazônica.

brunneus Fabricius, 1801:7 (Erotylus). Tp.: ZMCD - (América meridional). - Duponchel, 1825:174. - Lacordaire, 1842:516. - Zimsen, 1964:115. - Gemminger \& Harold, 1876:3720 (Homoeotelus). - Chevrolat, 1843: 80 (syn.).

lineaticollis Duponchel, 1825:167 (Erotylus). - Lacordaire, 1842:511 (syn.). 
peruvianus Kirsh, 1876:110 (Omoiotelus). Tp.: SMTD exam. aut. - (Peru, Pozuzu). - Gemminger \& Harold, 1876:3720 (Homoeotelus). - Kuhnt, 1909:49; 1911:35. - Deelder, 1942:71. - Mader, 1942d:169; 1951:204. Blackwelder, 1945:463. Distr.: Peru. Bolívia.

prioteloides Crotch, 1876:561 (Homoeotelus). Tp.: MCZC exam. aut. - (Amazonas). - Gemminger \& Harold, 1876:3720. - Kuhnt, 1909:50; 1911:36. Blackwelder, 1945:463. Distr.: Brasil, região amazônica.

rugosus Crotch, 1876:561 (Homoeotelus). Tp.: MCZC exam. aut. - (Amazonas). - Gemminger \& Harold, 1876:3720. - Kuhnt, 1909:50; 1911:36. - Blackwelder, 1945:463. - Guérin, 1948:18. Distr.: Brasil, região amazônica.

spinifer Thomson, 1857:410 (Omoiotelus). Tp.: MNHN exam. aut. - (Amazonas). - Crotch, 1876:561 (Homoeotelus). Gemminger \& Harold, 1876:3720. Kuhnt, 1909:50, pl. 1, figs 11, 11a; 1911:36. - Deelder, 1942:71. Blackwelder, 1945:464. - Guérin, 1948:18. Distr.: Peru. Brasil, região amazônica.

terminalis Erichson, 1847:177 (Homoeotelus). Tp.: ? - (Peru). - Crotch, 1876:560. - Gemminger \& Harold, 1876: 3720. - Kuhnt, 1909:49; 1911:36. - Deelder, 1942:71. - Mader, 1942d:169; 1951:204. - Blackwelder, 1945:464. Distr.: Peru. Bolívia.

testaceus Fabricius, 1775:822 (Erotylus). Tp.: ZMCD - (América); 1781:157; 1787:91; 1792:36; 1801:4. - Olivier, 1792:432. - Herbst, 1799:377. Schönherr, 1808:325. - Duponchel, 1825:60, pl. 7, fig. 58. - Sturm, 1826:139. - Zimsen, 1964:114. - Lacordaire, 1842:508 (Omoiotelus). Guérin-Méneville, 1855:612. - Kirsch, 1876:110. - Crotch, 1876:559 (Homoeotelus). - Gemminger \& Harold, 1876:3721. - Kuhnt, 1909:50; 1911:36. - Deelder, 1942:71. - Blackwelder, 1945:464. - Guérin, 1948:18.

Distr.: Colômbia. Peru. Brasil.

lugubris Gmelin, 1790:1727 (Erotylus). - Lacordaire, 1842:508 (syn.).

immaculatus Olivier, 1807:478, pl. 2, fig. 27 (Erotylus). - Lacordaire, 1842:508 (syn.).

umbonatus Lacordaire, 1842:510 (Omoiotelus). Tp.: MCZC exam. aut. - (Colômbia). - Crotch, 1876:559 (Homoeotelus). - Gemminger \& Harold, 1876: 3721. - Dohrn, 1878:452. - Kuhnt, 1909:50; 1911:36. - Deelder, 1942:71. - Blackwelder, 1945:464. - Delkeskamp, 1957:98. Distr.: Colômbia. Bolívia.

trimaculatus Kirsh, 1865:102 (Omoiotelus). - Crotch, 1876:559 (syn.).

vitiosus Crotch, 1876:560 (Homoeotelus). Tp.: MCZC exam. aut. - (Amazonas). - Gemminger \& Harold, 1876:3721. - Kuhnt, 1909:50; 1911:36. - Blackwelder, 1945:464. - Guérin, 1948:18. Distr.: Brasil, região amazônica. 


\section{Ellipticus (Gemellatotelus) Mader, 1942.}

Gemellatotelus Mader, 1942d:168 (Tsubgen. Omoiotelus gemellatus Lacordaire, 1842:513, desig. orig.). - Alvarenga, 1965:84.

acuminatus Gorham, 1891:56 (Homoeotelus). Tp.: NHML exam. aut. - (Equador, Nanegal). - Kuhnt, 1909:50 (Homoeotelus); 1911:35. - Deelder, 1942:70.

- Blackwelder, 1945:463. Distr.: Equador.

crocicollis Lacordaire, 1842:514 (Omoiotelus). Tp. ? - (Colômbia). - Gemminger \& Harold, 1876:3720. - Kuhnt, 1909:50; 1911:35. - Deelder, 1942:70. Blackwelder, 1945:463. Distr.: Colômbia.

gemellatus Lacordaire, 1842:513 (Omoiotelus). Tp. ? - (México). - Crotch, 1876:561 (Homoeotelus). - Gemminger \& Harold, 1876:3720. - Gorham, 1889:113, pl. 6, fig. 22. - Kuhnt, 1909:50; 1911:35. - Deelder, 1942:70. - Blackwelder, 1945:463. Distr.: México. América Central. Colômbia.

jansoni Crotch, 1873 b: 150 (Homoeotelus). Tp.: NHML exam. aut. - (Nicarágua, Chontales, Santo Domingo); 1876:561. - Gemminger \& Harold, 1876: 3720. - Gorham, 1889:114, pl. 6, fig. 25. - Kuhnt, 1909:50; 1911:35. Blackwelder, 1945:463. Distr.: Nicarágua. Panamá.

mexicanus Crotch, 1876:561 (Homoeotelus). Tp.: MCZC exam. aut. - (México). - Gemminger \& Harold, 1876:3720. - Gorham, 1889:114, pl. 6, figs 23, 24. - Kuhnt, 1909:50; 1911:35. - Blackwelder, 1945:463. Distr.: México. Guatemala. Panamá.

\section{Ellipticus (Calenus) Dejean, 1837.}

Calenus Dejean, 1837:427 (Tsubgen. Erotylus signaticollis Duponchel, 1825:159, seg. Duponchel, 1844:105). - Duponchel, 1844:105. - Alvarenga, 1965:82. Glabratotelus Mader, 1942d:168. - Alvarenga, 1965:84 (syn.).

nigrolimbatus Mader, 1942b:80 (Homoeotelus). Tp.: MGFM exam. aut. - (Brasil, Rio de Janeiro). Distr.: Brasil, região sudeste.

signaticollis Duponchel, 1825:159, pl. 7, fig. 66 (Erotylus). Tp.: MNHN exam. aut. - (Brasil), - Lacordaire, 1842:514 (Omoiotelus). - Crotch, 1876:562 (Homoeotelus). - Gemminger \& Harold, 1876:3720. - Kuhnt, 1909:50; 1911:36. - Blackwelder, 1945:463. - Guérin, 1948:18. Distr.: Brasil, regiões sudeste e central. 


\section{Ellipticus (Inflatotelus) Mader, 1942.}

Inflatotelus Mader, 1942d:168 (Tsubgen. Homoeotelus inflatus Mader, 1942:193, desig. orig.). - Alvarenga, 1965:85.

bolivianus Mader, 1942d:194 (Homoeotelus). Tp.: MGFM exam. aut. - (Bolívia, Coroico); 1951:205. Distr.: Bolívia.

inflatus Mader, 1942d:169, 193 (Homoeotelus). Tp.: MGFM exam. aut. - (Peru, Oxapampa). Distr.: Peru.

laevis Delkeskamp, 1957:106 (Homoeotelus). Tp.: ZMHU exam. aut. - (Peru, Huancabamba). Distr.: Peru.

marmoratus Delkeskamp, 1957:98, 105, fig. 6 (Homoeotelus). Tp.: ZSBS (Bolívia, Chapare). Distr.: Bolívia.

\section{Brachylon Gorham, 1898.}

Brachylon Gorham, 1898:256 (Tgen. Brachylon breve Gorham, 1899:257, monotipia). - Kuhnt, 1909:9, 51. - Deelder, 1942:51. - Alvarenga, 1965:82.

breve Gorham, 1899:257. Tp.: NHML - (México, Guerrero, Omilteme). - Kuhnt, 1909:51; 1911:36. - Blackwelder, 1945:464. Distr.: México. Nicarágua. 


\section{REFERÊNCIAS BIBLIOGRÁFICAS}

ACHARD, J. 1926. Fragments entomologiques (10). Nouveaux erotylides américains. Ann. Bull. Soc. ent. Belg., Bruxelles, 65 (suppl.): 143-145.

Alvarenga, M. 1962. Sobre uma espécie nova do gênero Coccimorphus Hope, 1841 (Coleoptera, Erotylidae). Atas Soc. Biol. Rio de Janeiro 6 (1-2):11-12. 1965. Espécies tipos dos gêneros e subgêneros neotropicais da família Erotylidae (Coleoptera). Bol. Univ. Fed. Paraná, Curitiba, 2 (6): 75-92.

. 1970. Adendas e corrigendas ao trabalho "Espécies tipos dos gêneros e subgêneros neotropicais da família Erotylidae (Coleoptera)". Atas Soc. Biol. Rio de Janeiro 12 (supl.): 45-46.

1971a. Descrição de uma espécie nova do gênero Eurycardius Lacordaire, 1842 (Coleoptera, Erotylidae). Atas Soc. Biol. Rio de Janeiro 14 (5-6): 117-118.

1971b. Descrição de uma espécie nova do gênero Erotylus Fabricius, 1775 (Coleoptera, Erotylidae). Atas Soc. Biol. Rio de Janeiro 14 (5-6): 149-150.

. 1971c. Kakoia travassosi gênero e espécie nova de Erotylidae (Coleoptera). Atas Soc. Biol., Rio de Janeiro, 15 (1): 35-36.

. 1976. Notas sobre algumas espécies neotropicais com nomes preocupados da família Erotylidae (Coleoptera). Studia ent., Rio de Janeiro, 19 (1-4): 511-513.

1977. Notas taxonômicas sobre a família Erotylidae (Coleoptera). Dusenia, Curitiba, 10 (2): 103-107.

1983. Seis espécies novas do gênero Mycotretus Chevrolat, 1837, da região amazônica. (Coleoptera, Erotylidae). Acta Amazônica, Manaus, 13 (3-4): 583-589.

1989. Uma espécie nova de Mycotretus Chevrolat, 1837, da região amazônica (Coleoptera, Erotylidae). Mem.Inst. Oswaldo Cruz, Rio de Janeiro, 84 (Supl. IV): 35-36.

ARrow, G.J. 1909. Systematic notes on Coleoptera of clavicorn families. Ann. Mag. nat. Hist., London, ser. 8, 4: 190-196.

-1937. Notes on some clavicorn Coleoptera and descriptions of few species and genera. Ann. Mag. nat. Hist., London, ser 10, 20 (10): 101-113.

BEDEL, L. 1919. Le Silpha indica Linné, consideré comme énigme, est un Megalodacne (Col. Erotylidae) de l'Amérique du Sud. Bull. Soc. ent. France, Paris, p. 114-116.

BERG, F.G.C. 1881. In: ROCA. Informes oficial de la Comision Cientifica agregada al Estado Mayor General de la expedicion al Rio Negro (Patagonia). Zoologia. Buenos Aires, 77-115p.

BLACKWELDER, R.E. 1945. Checklist of the Coleopterous insects of Mexico, Central America, the West Indies and South America. Bull. U.S. nat. Mus., Washington, 185 (3): 443-550.

BlanchaRd, C.E. 1843. In: Brullé. Insectes de l'Amérique méridionale 
recueillis par Alcides d'Orbigny. Paris, 2 (2).

Blatchley, W.S. 1917. On some new or noteworthy Coleoptera from the west coast of Florida. Canadian ent., Ontario, 49: 137-143.

Boyle, W.W. 1954a. Five news species of Triplax from western North America.

Proc. ent. Soc., Washington, 56 (5): 251-264.

. 1954b. Concerning the status of Ischyrus graphicus Lacordaire, with description of four new Erotylid species from western North America. J. New York ent. Soc. 62: 39-53.

1956. A revision of Erotylidae of America north of Mexico (Coleoptera).

Bull. Am. Mus. nat. Hist., Washington, 110: 61-172.

Böving, A.G. \& F.C. CRAighead. 1930-1931. An illustrated synopsis of the

principal larval forms of the order Coleoptera. Ent. Am., Brooklyn, new ser.,

11: 1-351.

Bréthes, J. 1920. Insectes du Perou. An. Soc. cient. argentina, Buenos Aires, 89: $27-54$.

BRuCH, C. 1914. Catálogo sistemático de los coleópteros de la Republica Argentina. 9. Rev. Mus. La Plata 19: 346-441.

BuQuet, J.B.L. 1840. Coléoptères nouveaux. Rev. Zool., Paris, p. 172-173.

CANDEZE, E.C.A. 1861. Histoire des métamorphoses de quelques coléoptères exotiques. Mém. Soc. R. sc., Liége, 16: 235-410.

Casey, T.L. 1916. Some random studies among the Clavicornia. Mem. Coleoptera, Lancaster, 7: 35-292.

- 1924. Additions to North American Coleoptera. Mem. Coleoptera, Lancaster, 11: 177-180.

Chagnon, G. 1933-1940. Contribution á 1'étude des coléoptères de province de Québec. Naturaliste Canadien, Montréal, (1-5): 1-385.

CHAPUIS, F. 1876. In: LACORDAIRE. Histoire naturelle des Insectes. Genéra de Coléoptères. Paris, 12: 1-424.

Chevrolat, L.A.A. 1834. Coléoptères du Mexique. Strasbourg, fasc. 1-8. . 1837. In: DEJEAN. Catalogue des coléoptères de la collection de M. le comte Dejean. 2e. éd., Paris, 5: 361-443. p.79-81.

CHÛ̉ô, M. \& M. CHÛ̉ô. 1988. A Catalog of the Erotylidae (Insecta, Coleoptera) from the old word (Excl. the Ethiopian region). Esakia, Hikosan, (26): 139-185.

Crotch, G.R. 1873a. Synopsis of the Erotylidae of Boreal America. Trans. Am. ent. Soc., Philadelphia, 4: 349-358.

1873b. A list of Erotylidae collected by Edward M. Janson, in vinicity of Santo Domingo, Chontales, Nicaragua, with descriptions of new genera and species. Cist. ent., London, 1: 141-150.

- 1876. A revision of the coleopterous family Erotylidae. Cist. ent., London, 1: 377-572. 
CurRan, C.H. 1941. Erotylidae of Kartabo, Barctica, British Guiana. Coleoptera. Zoologica: New York zool. Soc. 26 (3): 281-288.

1944. Notes and descriptions of some American Erotylidae. Am. Mus. Nov., New York, (1255): 1-14.

CURTIS, J. 1838. British entomology. London, 15: 674-721.

DEGEER, K. 1752-1778. Mémoires pour servir à 1'histoire des insectes. Stockholm, 5: 1-448.

4-5: $1-490$.

DeElder, C.L. 1942. Revision of Erotylidae (Col.) of the Leiden Museum. Zool. Meded. 24 (1-2): 49-115.

Dejean, P.F.M.A. 1837. Catalogue des coléoptères de la collection de M. le comte Dejean. 2e. éd., Paris, 5: 361-443.

DELKESKAMP, K., 1952 - Revision von 2 Untergattungen und Verzeichnis der Erotyliden-Arten von Angola (Col. Erotyl.). Publ. cult. comp. diam. Angola, Lisboa, 14: 55-90.

1957. Beiträge zur Kenntnis der Insektenfauna Boliviens. Teil 3, Col.

2, Erotylidae. Veröff. Zool. Staatssamml., München, 5: 93-116. . 1981. Coleopterorum Catalogus Supplementa; Erotylidae von Afri-

ka und Madagascar. Junk, Hague. Pars 34: 3-65.

Demay, 1838. Coléoptères nouveaux de la Guyane Française. Rev. Zool, Paris, p.22-24.

Dohrn, C.A. 1878. Exotisches. Stett. ent. Zgt., Stettin, 39: 444-462. 1880. Exotisches. Stett. ent. Zgt., Stettin, 41 (1-3): 149-157. 1883. Exotisches. Sttet. ent. Zgt., Stettin, 44 (1-3): 103-104.

Duméril, A.M.C. 1823. Considerations générales sur la classe des insectes. Paris, 1-343p.

Duponchel, P.A.J. 1825. Monographie du genre Erotyle. Mém. Mus. d'Hist. nat., Paris, 12: 30-61, 156: 176.

- 1844. In: D'ORBIGNY. Dictionaire Universel d'Histoire Naturelle. Paris, 3: 1-744.

Duponchel, P.A.J. \& L.A.A. Chevrolat. 1844. In: D’ORBIGNY. Dictionaire Universel d'Histoire Naturelle. Paris, 3: 1-744.

ERICHSON, W.F. 1847. Conspectus insectorum coleopterorum quae in Republica Peruana observata sunt. Arch. Naturg., Berlin, 13: 67-185. 533-617.

FABRICIUS, J.C. 1775. Systema entomologiae. Flensburg \& Leipzig, 1-832p. - 1776. Genera insectorum. Cologne, 1-310p.

- 1781. Species insectorum. Hamburgui, 1: 1-552. . 1787. Mantissa insectorum. Hafniae, 1: 1-348.

—. 1792. Entomologia systematica. Hafniae, 1 (2): 330-538. 1798. Supplementum entomologiae systematica. Hafniae, 1-572p. 
1801. Systema eleutheratorum. Kiel, 1: 1-505, 2: 1-687.

Fauvel, C.A.A. 1860. Catalogue des insectes recueillis à la Guyane Française.

Bull. Soc. linn. Normandie, Caen, 5: 299-327.

Fleutiaux, E. 1886. Supplement au catalogue des coléoptères de MM. Gemminger et Harold (langurides et érotylides). Ann Soc. ent. Belg., Bruxelles, 30: $216-224$.

Froeschner, R.C. \& E.P. Meiners. 1953. The Languridae and Erotylidae (Coleoptera) of Missouri with notes and keys. J. Kansas ent. Soc., Kansas City, 26: 18-25.

Gemminger, M. \& E. Harold. 1876. Catalogus coleopterorum. Monachii, 12: 3479-3822.

Germar, E.F. 1824. Coleopterorum species novae. Halae, 1-624p.

GisTEL, J.N.F.X. 1848. Naturgeschichte des Thierreichs fur höhere Schulen, Stuttgart, 1-216p.

- 1857. Achthundert und zwanzig neue oder unbeschriebene wirbellose Thiere. Straubing, 1-94p.

GMELIN, J.F. 1788. Linné's systema naturae. Ed. 13, 1 (4): 1517-2224.

GORHAM, H.S. 1883. Descriptions of new species of beetles belonging to the family Erotylidae. Proc. zool. Soc., London, 1:75-87.

. 1887-1889. In: Biologia Centrali-Americana, Insecta. Coleoptera. Erotylidae. Endomychidae and Coccinelidae. 7: 33-114.

. 1889. Descriptions of new species of the coleopterous, family Erotylidae. Proc. zool. Soc., London, p.613-620.

. 1891. In: WHYMPER. Coleoptera. Supplementary appendix to travels amongst the Great Andes of the Equator. London, 22-147p.

7: $253-257$.

GuÉrin, J. 1946a. Dois novos Erotylidae da América do Sul. Rev. Ent., Rio de Janeiro, 17 (1-2): 274-276.

1946b. Notas informativas e descrição de novas especies de Erotylidae.

Rev. bras. Biol., Rio de Janeiro, 6 (3): 365-372.

. 1948. Catálogo dos Erotylidae das coleções do Instituto de Ecologia e Expe- rimentação Agrícolas do Ministério da Agricultura, com a descrição de algumas espécies novas. Bol. Inst. Ecol. Exp. Agr., Rio de Janeiro, 8: 1-26.

1949a. Descrição de novas espécies neotropicais das famílias Clytridae, Megalopodidae e Erotylidae. (Col.). Rev. Ent., Rio de Janeiro, 20 (1-3): 229-236.

1949b. "Erotylidae" argentinas. Description de una especie nueva del genero Mycotretus. Acta zool. lilloana, Tucumán, 7: 589-591.

. 1952. Essay monographique des erotyliens chiliens (Coleoptera). Rev. chil. Ent., Santiago, 2: 179-183.

1956. Descrição de novas espécies e variedades de Erotylidae (Col.)

neotropicais. Dusenia, Curitiba, 7 (1): 45-66. 
GuÉRIN-MÉnEville, F.E. 1841a. Description de quelques belles espéces d'érotyles. Rev. Zool., Paris, p.115-120.

- 1841b. Description de quelques espéces d'érotylides, extraite du manuscrit du texte explicatif de l'iconographie du règne animal. Rev. zool., Paris, p. 153-161.

- 1844. In: G. CUVIER. Iconographie du règne animal. Paris, 2: $1-576 \mathrm{p}$.

1855. Catalogue des insectes coléopterès recueillis par Gaetano Osculati, pendant son exploration de la région équatoriale, sur les bords du Napo e l'Amazone. Verh. zool- bot. Ges., Wien, 5: 573-612.

Harold, E. 1876. Geänderte Namen. Col. Hefte, 15, 174p.

Heller, K M. 1918a. Zur klassifikation einiger afrikanischer Erotyliden. Ent. Bl., Berlin, 14 (4-6): 136-157.

- 1918b. Ergäzungen zu meiner "Klassifikation einiger afrikanischer Erotyliden". Ent. Bl., Berlin, 14 (10-12): 274-288.

. 1920. Beitrag zur Kenntnis der Erotyliden de indo-australischen Region mit besonderer Berücksichtigung de philipischen Arten. Arch. Naturg., Berlin, 84: 1-121.

HERBST, J.F.W. 1783. Kritisches Verzeichniss meiner Insecten-Sammlung. Arch. Ins., Zurich, 4: 1-68.

- 1786. Erst Mantisse zum Verzeichniss de ersten Klasse meiner InsectenSammlung. Arch. Ins., Zurich, 7-8: 153-182.

- 1793. Natursyst. Käfer., Berlin, 5: 1-392.

- 1799. Natursyst. Käfer,, Berlin, 8: 1-420.

Hope, F.W. 1841. Observations sur les érotyles, avec description de plusieurs nouveaux genres et quelques espéces inédites. Rev. Zool., Paris, p. 109-115.

Houtturn, M. 1766. Natuurkundige beschrijving der Insekten. deel 7 , Amsterdam.

ILliger, J. 1804. Bemerkungen zum zweiten Theile von Fabricius systema eleutheratorum. Mag. Ins., Braunschweig, 3: 160-180.

-1806. Nachtrag zu den Zusätzen, Bemerkungen und Berichtigungen zu Fabricii systema eleutheratorum. Mag. Ins., Braunschweig, 5: 221-246.

Jacquelin du Val, P.N.C. 1857. In: SAGRA. Histoire Physique, Politique et

Naturelle de 1'ile de Cuba. Insectes. Ordre des coléoptères. Paris, 7: 137-328.

JOHNSON, D.H. 1967. Neotropical species of genus Triplax Herbst and revision of genus Haematochiton Gorham (Coleoptera, Erotylidae). Proc. U. S. nat. Mus., Washington, 123 (3601): 1-25.

KIRSCH, T.F.W. 1865. Beiträge zur Fauna von Bogota. Berl. ent. Z., Berlin, 9: 40-104.

\section{Z., Berlin, 20: 81-133.} 187-213. 
Kolbe, H.J. 1907. In: Erghnisse der Hamburger Magalhaensische Sammelreise. Coleopteren. Hamburg, 8 (4): 1-125.

Kunnt, P. 1908a. Synopsis der Gattungen Erotylus, Cypherotylus, Micrerotylus

(Col). Deut. ent. Z., Berlin, p.67-100, 225-238.

- 1908b. Neue Erotyliden (Col.). Deut. ent. Z., Berlin, p.626-633.

$1-139$. 1909. In: WYTSMAN. Genera Insectorum. Erotylidae. Bruxelles. 88:

1910. Neue Erotyliden (Col.). Deut. ent. Z., Berlin, p.219-270.

1911. In: JUNK \& SCHENKLING. Coleopterorum Catalogus. Erotylidae. Berlin, 34: 1-103.

LaCORdaire, J.T. 1842. Monographie des Erotyliens. Paris, p.1-543.

LAPORTE, F.L.M., comte de CASTELNAU. 1840. Histoire naturelle des insectes coléoptères. Paris, 2: 1-564.

Latreille, P.A. 1796. Précis des caractères génériques des insectes. Paris, p. 1-201.

1804. Histoire naturelle, général et particulière, des crustaces et de insectes. Paris, 12: 1-415.

1807. Genera crustaceorum et insectorum. Paris, 3: 1-258.

. 1812-1813. Insectes de 1'Amerique équinoxiale, recueillis pendant le voyage de MM. de Humboldt et Bonpland. In: Voyage de Humboldt et Bonpland. 10e. partie. Observations de zoologie et anatomie comparée. Paris, 1: 127-252, 2: 1-54.

LeConte, J.L. 1856. Synopsis of the Erotylidae of the United States. Proc. Acad. nat. hist., Philadelphia, 7: 158-163.

LENG, C.W. 1920. Catalogue of the Coleoptera of America, north of Mexico. Mt. Vernon, p. 1-470.

Linnaeus, C. 1758. Systema naturae. Ed. 10. Stockholm, 1: 1-823.

. 1763. Centuria insectorum rariorum. Upsala, 1-32p.

. 1767. Systema naturae. Ed. 12. Holmiae, 1 (2): 533-1327.

Mader, L. 1934. Coleopterologische Notizen, II. Ent. Anz., Wien, 14: 39-40. . 1935. Neue Coleopteren aus Süd-und Zentralamerika, I, II, III. Ent. Anz., Wien, 15: 241-244, 270-272, 293-295.

112-115. 14-19. 12-13. 32 (1): 219-222.

1942b. Zwei neue südamerikanische Erotylidae. Stett. ent. Ztg., Stettin, 103 (1): 79-81.

1942c. Zur kenntnis der amerikanische Erotyliden. Mitt. münch. ent. 
Ges., München, 32 (2-3): 549-572. 149-201.

- 1942d. In: TITSCHACK. Beiträge zur Fauna Perus. Hamburg, 2:

. 1943. Zur kenntnis der amerikanische Erotyliden. Stett. ent. Zgt., Stettin, 104: 109-116.

94-117.

1944. Die Erotyliden von Peru (Col.). Stett. ent. Zgt., Stettin, 105:

1951. Die Erotyliden von Peru (Col.). Ent. Arb. Mus. G. Frey, München, 2 (1): 197-225.

1955. Zwei neue Coleopteren aus Südamerika. Mitt. münch. ent. Ges., München, 54-55: 476-478.

Motschulsky, V. 1859. Insectes des Indes Orientales, et de contrées analogues. Helsingfors, Etud. ent. 8: 25-118.

Olivier, A.G. 1792. Encyclopédie méthodique. Histoire naturelle. Insectes. 6 (2): $369-704$.

- 1807. Entomologie, ou histoire naturelle des insectes. 5: 1-612.

Pallister, J.C. 1955a. The pleasing fungus beetles of north central Mexico, collected on the David Rockefeller Mexican expedition of 1947 (Coleoptera, Erotylidae). Am. Mus. Nov., New York, (1703): 1-6.

. 1955b. The pleasing fungus beetles collected on the explorers ClubAmerican Musen of Natural History entomological expedition to Yucatan, Mexico, in 1952 (Coleoptera, Erotylidae). Am. Mus. Nov., New York (1745): 1-9.

PAYKUll, J. 1800. Fauna Suecica; Insecta (Coleoptera). Upsala, 3: 1-459.

Percheron, A.R. 1835. Genera des insectes. Paris, livre 4, no. 6.

Peterson, A. 1951. Larvae of insects: Coleoptera, Diptera, Neuroptera,

Siphonaptera, Mecoptera, Trichoptera. Colombus, 2: 1-410.

Perty, J.A.M. 1832. Delectus animalium articulatorum in Brasilia collec-

torum. J. B. Spix et C. F. Martius collegerunt. Monachii, 2: 61-124.

Philippi, F. 1887. Catálogo de los Coleópteros de Chile. An. Univ. Chile, Santiago, 71: 619-791.

PhilipPI, R.A. \& F. PHILIPPI. 1864. Beschreibung einiger neuen chilenischen Käfer. Stett. ent. Zgt., Stettin, 25 (10-12): 313-406.

SCHAEFFER, C.F.A. 1905. Some additional new genera and species of Coleoptera found within the limit of the United States. Bull. Mus. Brooklyn, New York, 1: $141-179$. Soc., 23: 235-238.

Schenkling, S. 1919. Erotyliden-Studien I. Arch. Naturg., Berlin, 83A (11): 77-93.

SCHÖNHERR, C.J. 1808. Synonimia insectorum. Stockholm, 1 (2): 1-624.

STAIG, R.A. 1931. The Fabrician types of insects in the Hunterian collection at Glasgow University. Glasgow Univ. Publ. 19 (1): I-XVI+1-110. 
STURM, J. 1826. Catalog meiner Insecten-Sammlung. Käfer. Nürnberg, 1: 1-207. 1843. Catalog der Käfer-Sammlung. Nürnberg, 1-386p.

Sulzer, J.H. 1761. Die Kennzeichen der Insekten. Zuricii, 1-203p.

- 1776. Abgekurzte geschichte der Insecten nach dem linaeischen System. Winterhur, 1: 1-274, 2: 1-32.

TAschenberg, E.L. 1870. Neue Käfer aus Columbien und Ecuador. Z. Ges. Naturw., Berlin, 35: 177-199.

Thomson, J. 1856. Description de dix-sept coléoptères. Rev. Mag. Zool., sér. 2, Paris, 8: 472-483.

- 1857. Description de dix coléoptères. Arch. Ent., Paris, 1: 399-411.

THUNBERG, C.P. 1801. Dissertatio sistens novae insectorum species. Gottingen, 3: $124-248$.

VOET, J.E. 1778. Catalogus systematicus coleopterorus. Haye, 2: 1-82.

WATERHOUSE, C.O. 1877. Descriptions of new Coleoptera from Medellin, Colombia. Cist. ent., London, 2: 421-429.

WiCKMAN, H.F. 1894. The Coleoptera of Canada. VI. The Endomychidae and Erotylidae of Ontario and Quebec. Canadian ent., Ontario, 26: 337-342.

Zimsen, E. 1964. The type material of I. C. Fabricius. Copenhagen, 1-656p. 


\section{ÍNDICE}

abdominalis Kuhnt, 1908 . . . . . . . . . . . . . . . . . . 57

abdominalis Oliv., $1792 \ldots \ldots$. . . . . . . . . . . . . . . . . . . . 69

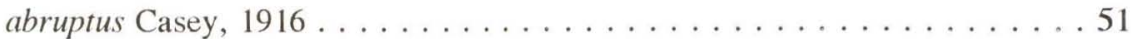

Acronotus Lac., $1842 \ldots \ldots \ldots$. . . . . . . . . . . . . . . . 67

acuminatus Gorham, $1891 \ldots \ldots \ldots \ldots \ldots \ldots \ldots \ldots \ldots \ldots$

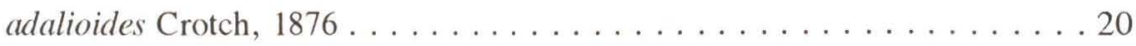

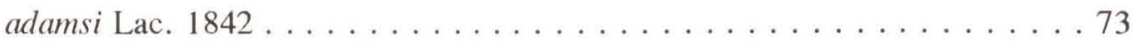

adrianae Alv., $1976 \ldots \ldots \ldots \ldots 1$

adustus Dup. $1825 \ldots \ldots \ldots \ldots \ldots$. . . . . . . . . . . . . . 79

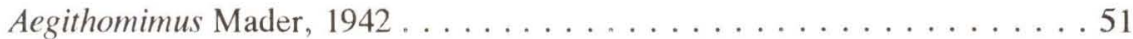

Aegithomorphus Lac., 1842 . . . . . . . . . . . . . . . . . . . . . 72

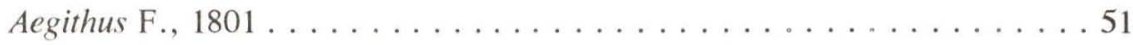

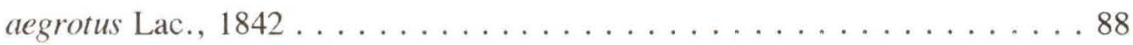

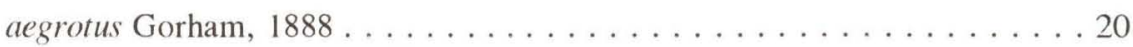

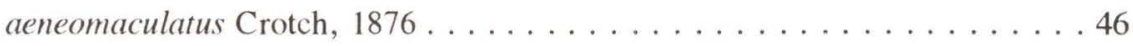

aeneoniger Crotch, $1876 \ldots \ldots \ldots \ldots \ldots 1$

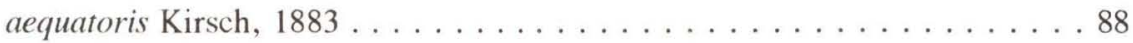

aestuans Lac. $1842 \ldots \ldots \ldots \ldots$. . . . . . . . . . . . . . . 20

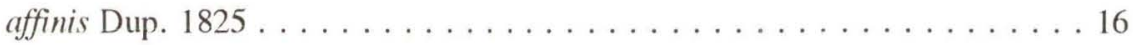

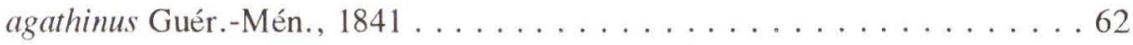

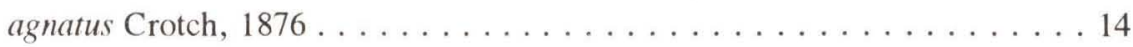

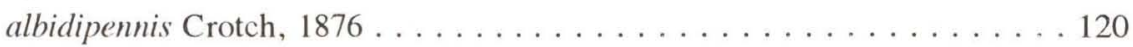

albidoguttatus Crotch, $1876 \ldots \ldots \ldots \ldots \ldots \ldots \ldots$

alboniger Guérin, $1956 \ldots \ldots \ldots \ldots$

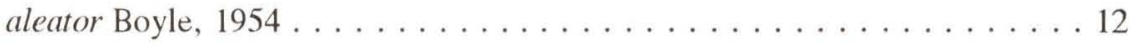

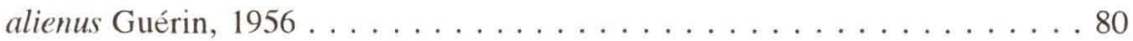

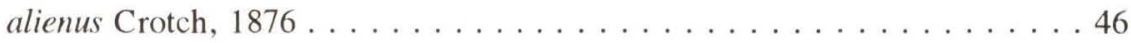

Alloiotelus Hope, 1841 . . . . . . . . . . . . . . . . . . . . 107

alternans Gorham, $1888 \ldots \ldots \ldots \ldots \ldots$

alternans Oliv., $1792 \ldots \ldots \ldots \ldots \ldots$. . . . . . . . . . . . . 107

alutaceus Erichson, $1847 \ldots \ldots \ldots \ldots \ldots \ldots \ldots$

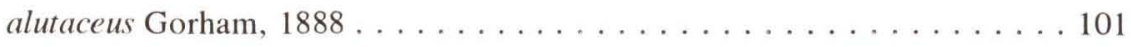

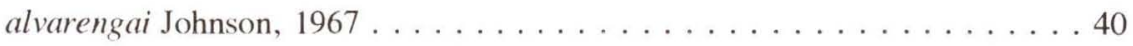

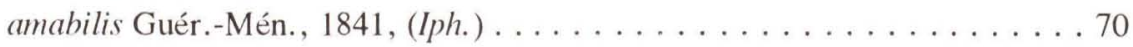


amabilis Guér.-Mén., 1841, (Meg.) . . . . . . . . . . . . . . . 61

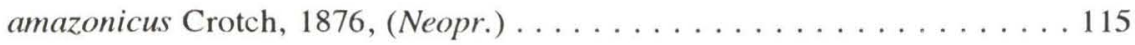

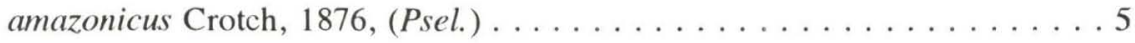

amazonus Crotch, $1876 \ldots \ldots \ldots \ldots \ldots \ldots \ldots$

ambiguus Lac., $1842 \ldots \ldots \ldots \ldots \ldots$

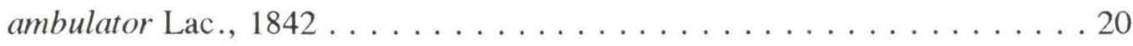

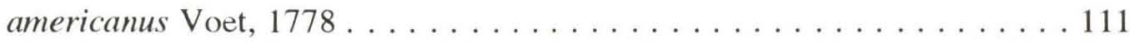

amictus Erichson, $1847 \ldots \ldots \ldots \ldots \ldots \ldots$

amoenus Guér.-Mén., $1841 \ldots \ldots \ldots \ldots \ldots \ldots \ldots \ldots$

amoenus Mader, $1942 \ldots \ldots \ldots \ldots \ldots \ldots \ldots \ldots \ldots \ldots \ldots \ldots \ldots$

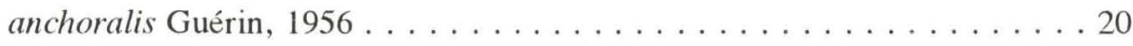

andicola Kirsch, $1876 \ldots \ldots \ldots \ldots \ldots \ldots$

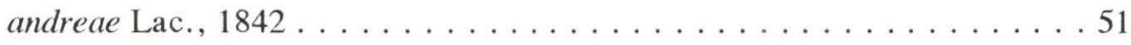

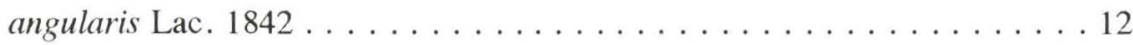

angustatus Lac., $1842 \ldots \ldots \ldots \ldots \ldots \ldots \ldots \ldots$

anisotomoides Gorham, $1888 \ldots \ldots \ldots \ldots \ldots \ldots \ldots \ldots \ldots \ldots \ldots$

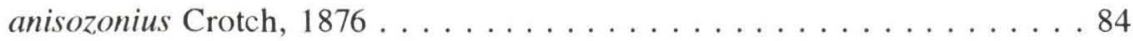

annularis Lap., $1840 \ldots \ldots \ldots$. . . . . . . . . . . . . . . . . 67

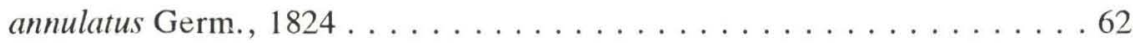

annulatus Lac., $1842 \ldots \ldots \ldots \ldots \ldots$. . . . . . . . . . . . 102

annulipes Guér.-Mén., 1841 . . . . . . . . . . . . . . . . . . 101

anteinterrupta Mader, $1942 \ldots \ldots \ldots$. . . . . . . . . . . . . . . 79

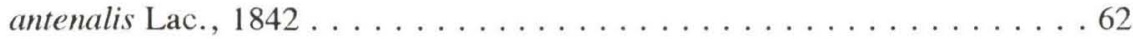

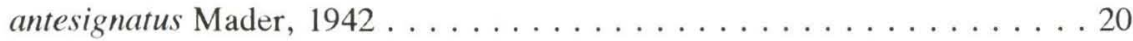

anthracinus Gorham, $1888 \ldots \ldots \ldots \ldots 2 \ldots \ldots \ldots \ldots$

apiatoides Mader, $1942 \ldots \ldots \ldots \ldots \ldots \ldots \ldots \ldots \ldots \ldots \ldots \ldots$

apiatus Lac., $1842 \ldots \ldots \ldots \ldots \ldots \ldots \ldots \ldots$

apiatus Chevr., $1835 \ldots \ldots \ldots \ldots \ldots \ldots \ldots \ldots \ldots \ldots \ldots \ldots \ldots$

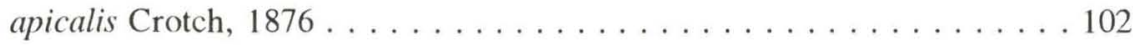

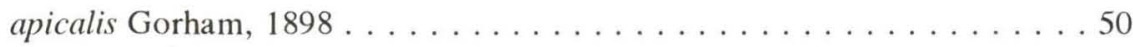

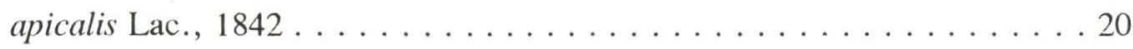

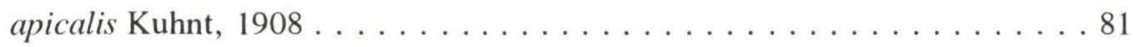

apicatus Kuhnt, $1910 \ldots \ldots \ldots \ldots \ldots \ldots \ldots \ldots \ldots \ldots \ldots \ldots$

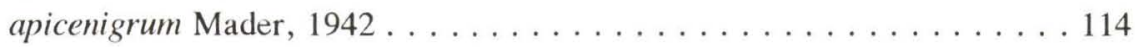

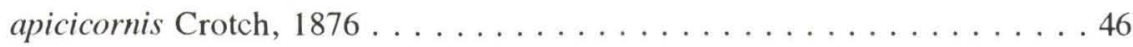

apicicornis Guér.-Mén., 1844 . . . . . . . . . . . . . . . . . . 120

Revta bras. Zool. 11 (1): 1 - 175, 1994 


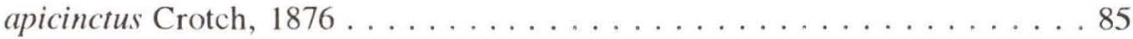

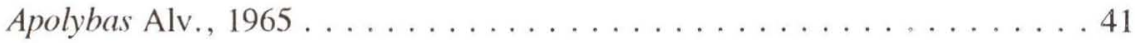

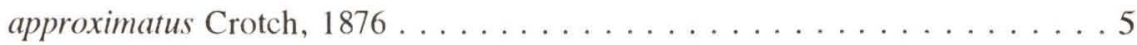

arcuatus Lac., $1842 \ldots \ldots \ldots \ldots$. . . . . . . . . . . . . . . 20

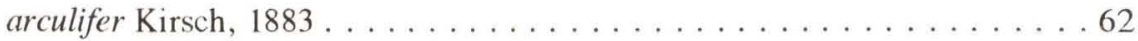

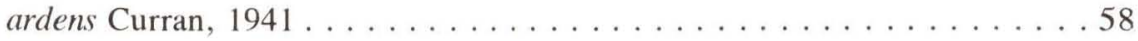

arduus Erichson, $1847 \ldots \ldots \ldots \ldots$. . . . . . . . . . . . . . . . 119

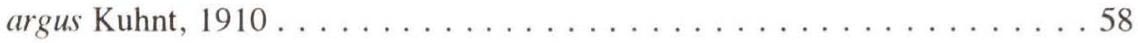

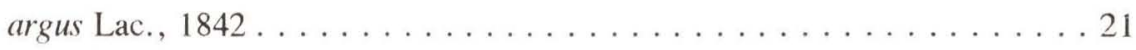

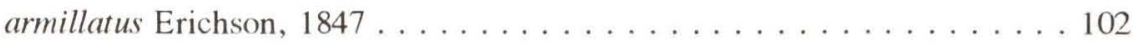

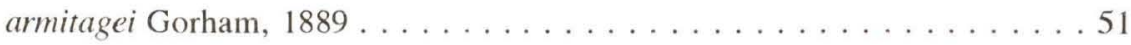

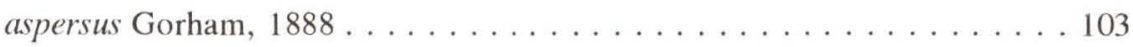

assequens Mader, $1942 \ldots \ldots \ldots \ldots \ldots \ldots \ldots$

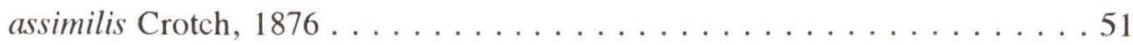

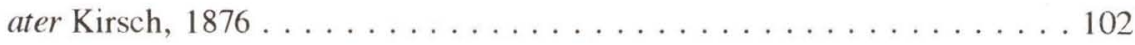

aterrimus Kirsch, $1876 \ldots \ldots \ldots \ldots \ldots \ldots$

atratus F., $1801 \ldots \ldots \ldots \ldots \ldots$

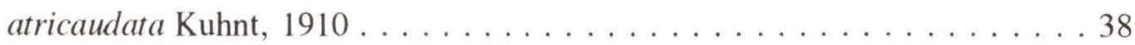

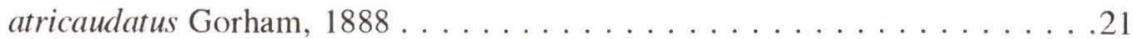

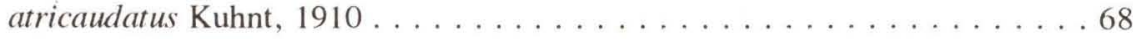

atricinctus Gorham, $1898 \ldots \ldots \ldots \ldots \ldots$. . . . . . . . . . . 50

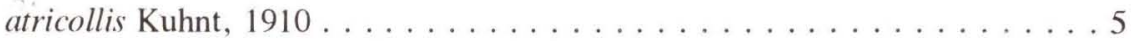

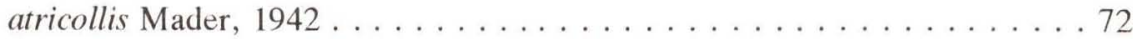

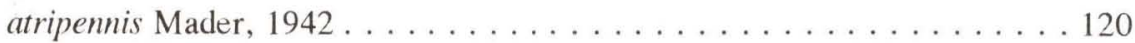

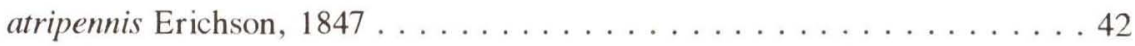

atrisignatus Alv., $1976 \ldots \ldots$. . . . . . . . . . . . . . . . . . 69

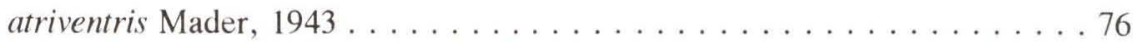

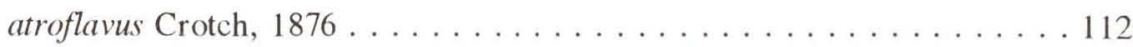

atrotibialis Alv., $1976 \ldots \ldots \ldots \ldots$

audouini Lac., $1842 \ldots \ldots \ldots \ldots \ldots \ldots \ldots \ldots$

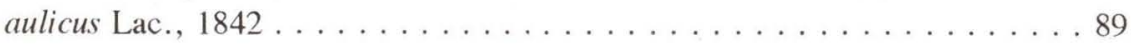

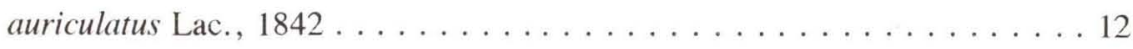

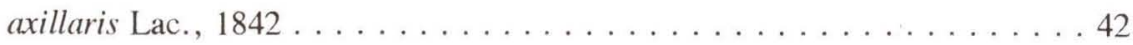

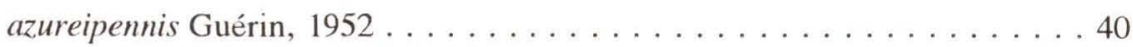

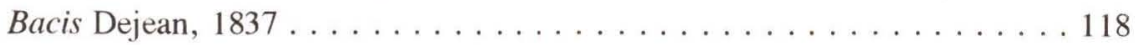

badeni Dohrn, $1883 \ldots \ldots \ldots . \ldots \ldots 2$ 


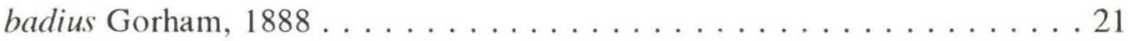

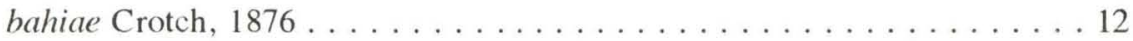

bajulus Lac., $1842 \ldots \ldots \ldots \ldots \ldots$. . . . . . . . . . . . . . . 79

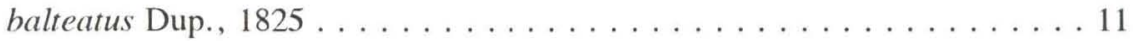

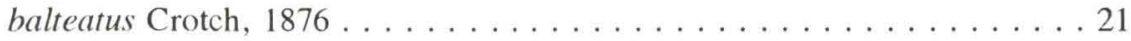

banghaasi Mader, $1935 \ldots \ldots \ldots \ldots \ldots 2$

bardia Curran, $1944 \ldots \ldots \ldots \ldots \ldots$. . . . . . . . . . . . . . . 98

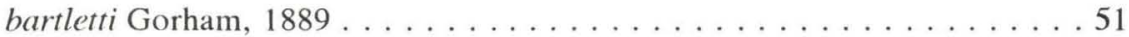

bartletti Gorham, $1883 \ldots \ldots \ldots \ldots \ldots \ldots \ldots$

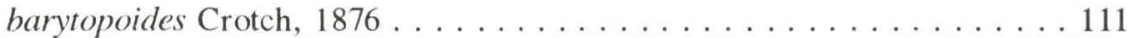

Barytopus Chevr., $1837 \ldots \ldots \ldots \ldots$. . . . . . . . . . . . 106

Barytopus Lac., 1842 . . . . . . . . . . . . . . . . . . . . . . . . 79

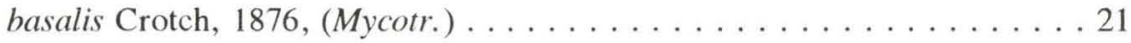

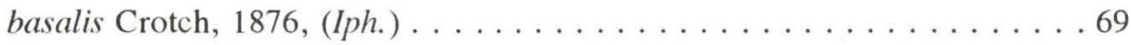

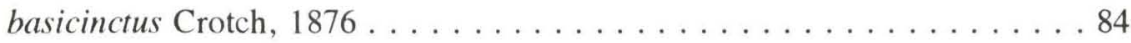

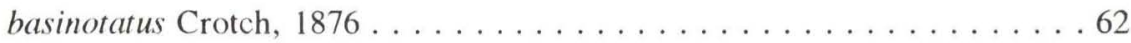

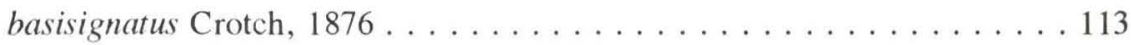

bassleri Curran, $1944 \ldots \ldots \ldots \ldots \ldots$. . . . . . . . . . . . 98

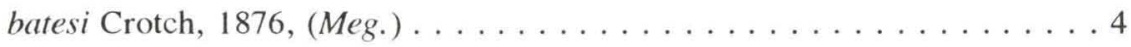

batesi Gorham, 1889 . . . . . . . . . . . . . . . . . . . . . 79

batesi Crotch, 1876, (Phric.) . . . . . . . . . . . . . . . . . 119

beauvoisi Lac., $1842 \ldots \ldots \ldots \ldots \ldots$. . . . . . . . . . . . . . 46

beebei Curran, $1941 \ldots \ldots \ldots \ldots \ldots$

bellicosus Lac., $1842 \ldots \ldots \ldots \ldots \ldots \ldots \ldots \ldots$

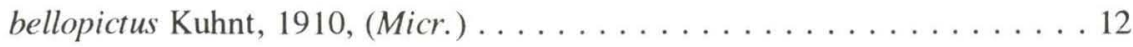

bellopictus Kuhnt, 1910, (Erot.) . . . . . . . . . . . . . . . . . 89

bellulus Lac., $1842 \ldots \ldots \ldots \ldots$. . . . . . . . . . . . . . . . 79

bellus Guérin, $1949 \ldots \ldots \ldots \ldots \ldots$. . . . . . . . . . . . . . . 12

bibalteatus Alv., 1976 . . . . . . . . . . . . . . . . . . . . . . 89

bicinctus Oliv., 1807 . . . . . . . . . . . . . . . . . . . . . . . . 79

bicinctus Guérin, 1949 . . . . . . . . . . . . . . . . . . . . . 21

bicinctus Guérin, $1948 \ldots \ldots \ldots \ldots$. . . . . . . . . . . . . . . 94

bicolor Lac., $1842 \ldots \ldots \ldots \ldots \ldots \ldots \ldots \ldots$

bicolor Guér.-Mén., $1841 \ldots \ldots \ldots$. . . . . . . . . . . . . . . 42

bicolor Lap., 1840 . . . . . . . . . . . . . . . . . . . . . . . . . . 87

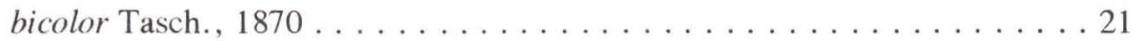


bicolor Guérin, $1952 \ldots \ldots \ldots \ldots \ldots \ldots$

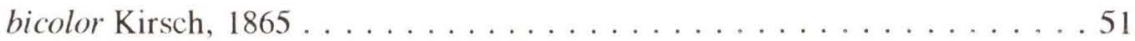

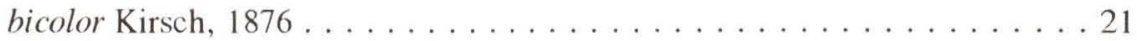

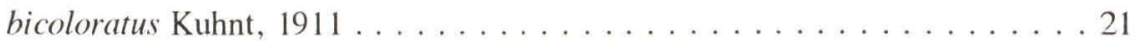

bifasciatus Guérin, $1952 \ldots \ldots \ldots \ldots \ldots \ldots \ldots \ldots$

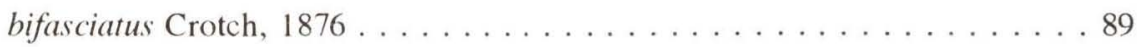

bifasciatus Herbst, $1799 \ldots \ldots \ldots \ldots$. . . . . . . . . . . . 108

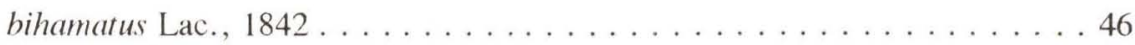

bilineatus Dup., $1825 \ldots \ldots \ldots \ldots \ldots \ldots \ldots \ldots \ldots \ldots \ldots$

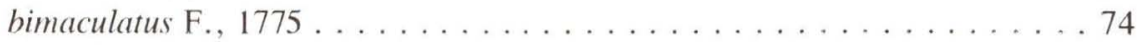

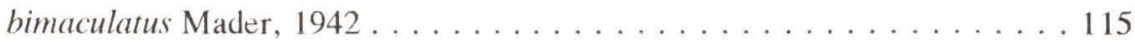

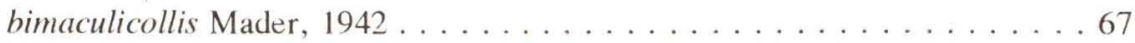

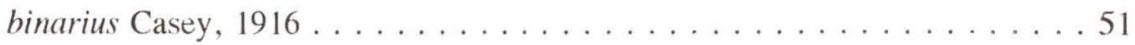

biplagiatus Guér.-Mén., $1841 \ldots \ldots \ldots \ldots \ldots \ldots \ldots \ldots$

bipunctatus Gorham, $1888 \ldots \ldots \ldots \ldots \ldots \ldots \ldots \ldots \ldots \ldots$

bisbimaculatus Lac.,1842 . . . . . . . . . . . . . . . . . . . 46

bisculptum Casey, $1916 \ldots \ldots \ldots$. . . . . . . . . . . . . . 39

bisigillatus Lac., $1842 \ldots \ldots \ldots \ldots$. . . . . . . . . . . . . . . . . 74

bisignatus Guér.-Mén., $1841 \ldots \ldots \ldots \ldots$. . . . . . . . 68

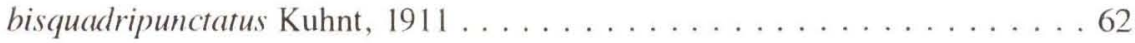

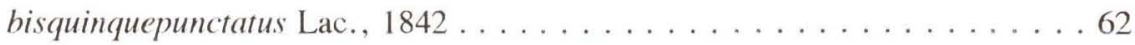

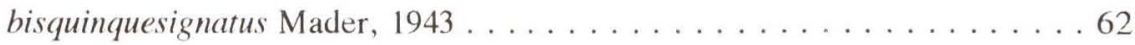

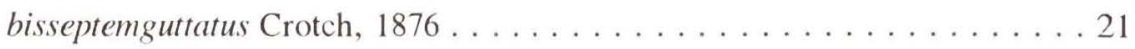

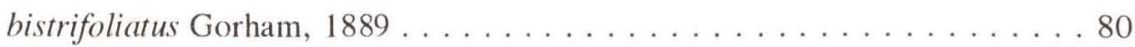

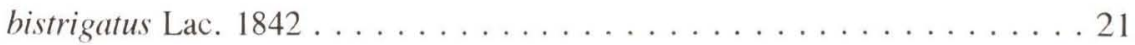

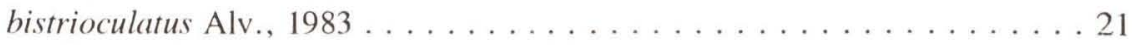

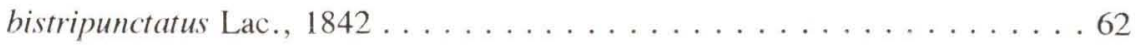

bitaeniatus Lac. $1842 \ldots \ldots \ldots \ldots \ldots \ldots \ldots \ldots \ldots \ldots \ldots \ldots$

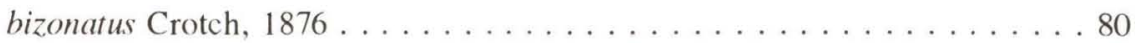

blandus Erichson, $1847 \ldots \ldots \ldots$. . . . . . . . . . . . . . 19

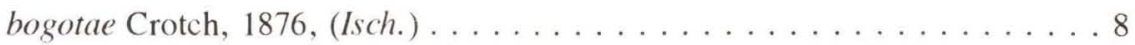

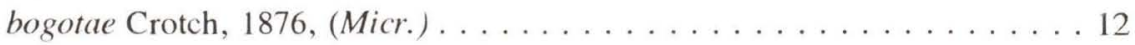

boisduvali Chevr., $1834 \ldots \ldots \ldots \ldots 2$

bolivianus Mader, 1942, (Gibb.) . . . . . . . . . . . . . . . 102

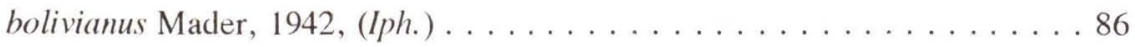

bolivianus Schenkl., $1919 \ldots \ldots \ldots$. . . . . . . . . . . . . . . 49 


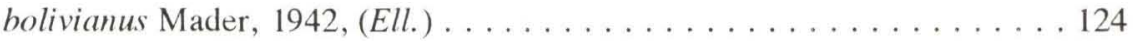

boliviensis Delk., $1957 \ldots \ldots . . \ldots \ldots$

bonplandi Lac., $1842 \ldots \ldots \ldots \ldots \ldots$

borgmeieri Alv., $1976 \ldots \ldots \ldots \ldots 2$

bosci Guér.-Mén., 1841 . . . . . . . . . . . . . . . . . . . . . . 110

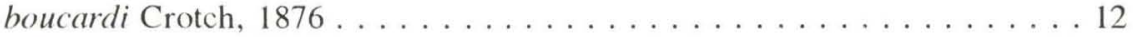

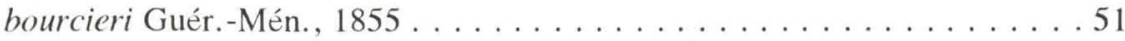

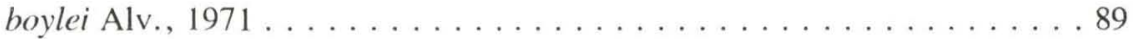

Brachylon Gorham, 1898 . . . . . . . . . . . . . . . . . . . . . 124

brachymeroides Crotch, $1876 \ldots \ldots \ldots \ldots \ldots \ldots \ldots$

Brachymerus Chevr., 1837 . . . . . . . . . . . . . . . . . . . . . 79

Brachysphaenus Khunt, 1909 . . . . . . . . . . . . . . . . . . . 58

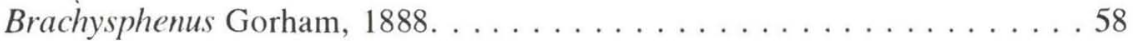

Brachysphoenus Lac., 1842. . . . . . . . . . . . . . . . 58

brasilianus Kuhnt, $1908 \ldots \ldots \ldots \ldots$. . . . . . . . . . . . 99

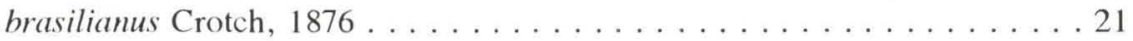

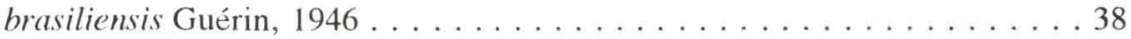

brasiliensis Lac., 1842, (Isch.) . . . . . . . . . . . . . . . . .9

brasiliensis Deelder, $1942 \ldots \ldots \ldots \ldots$

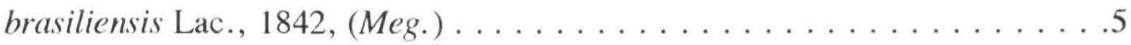

bremei Guér.-Mén., $1841 \ldots \ldots \ldots \ldots$. . . . . . . . . 80

breve Gorham, 1899 . . . . . . . . . . . . . . . . . . . . . . 124

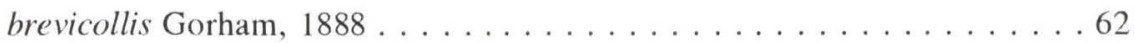

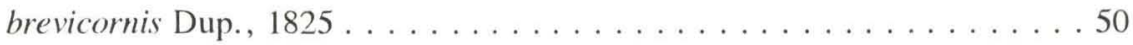

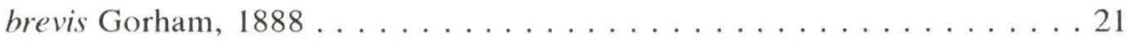

brongniarti Lac., $1842 \ldots \ldots \ldots \ldots \ldots$. . . . . . . . . . . . 80

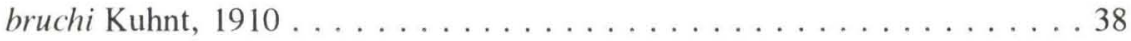

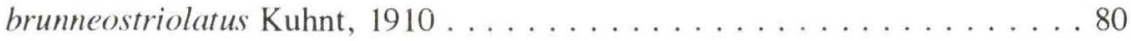

brunneus Kuhnt, 1908 . . . . . . . . . . . . . . . . . . . . 57

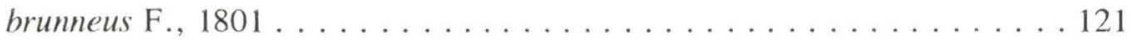

brunnipennis Lac., $1842 \ldots \ldots \ldots \ldots \ldots \ldots \ldots \ldots \ldots \ldots \ldots \ldots \ldots \ldots$

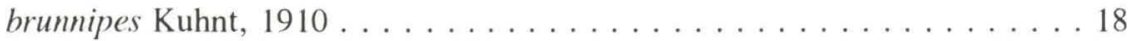

buckleyi Crotch, 1876, (Erot.) . . . . . . . . . . . . . . . . . 89

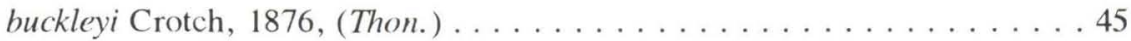

buckleyi Crotch, 1876, (Olig.) . . . . . . . . . . . . . . . . 107

bulla Lac., $1842 \ldots \ldots \ldots \ldots \ldots \ldots$. . . . . . . . . . . . . . . 51 
buqueti Lac., $1842 \ldots \ldots \ldots \ldots \ldots \ldots \ldots \ldots \ldots \ldots$

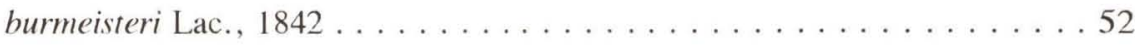

cacicus Lac., $1842 \ldots \ldots \ldots \ldots \ldots$. . . . . . . . . . . . . . 107

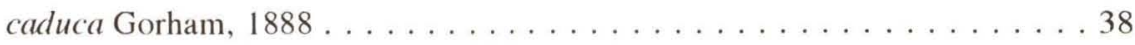

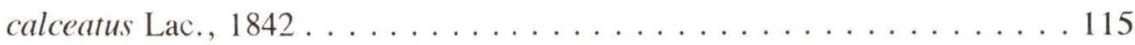

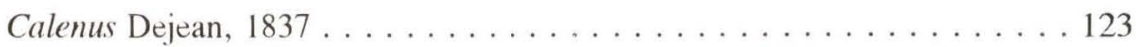

calidus Lac., $1842 \ldots \ldots \ldots \ldots \ldots \ldots$. . . . . . . . . . . . . . . 42

californica Horn, $1870 \ldots \ldots \ldots \ldots \ldots \ldots \ldots$

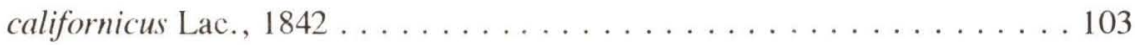

Callischyrus Crotch, $1876 \ldots \ldots \ldots \ldots \ldots \ldots \ldots$

camelus Guér.-Mén., $1841 \ldots \ldots \ldots$. . . . . . . . . . . . . . 103

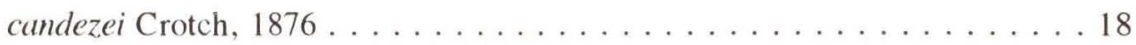

capitatus Lac., $1842 \ldots \ldots \ldots \ldots \ldots \ldots$. . . . . . . . . . . 49

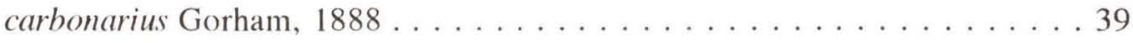

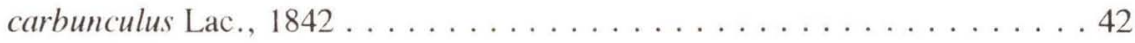

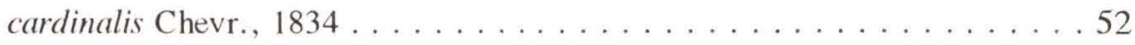

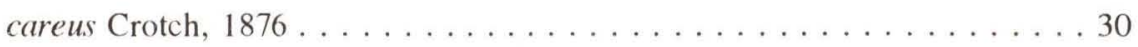

carmineus Lac., $1842 \ldots \ldots \ldots \ldots$. . . . . . . . . . . . . . 49

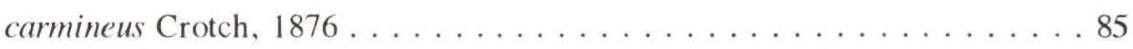

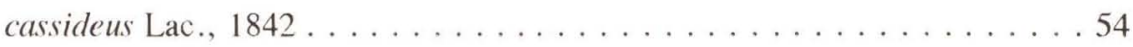

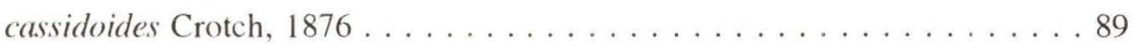

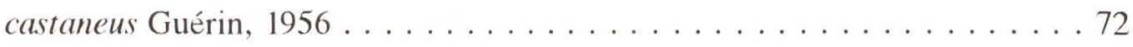

castaneus Gorham, $1888 \ldots \ldots \ldots \ldots \ldots \ldots \ldots \ldots \ldots$

catenatus Kuhnt, $1908 \ldots \ldots \ldots \ldots$. . . . . . . . . . . . 89

catenatus Crotch, $1876 \ldots \ldots \ldots \ldots \ldots \ldots \ldots$

catenulatus Lac., $1842 \ldots \ldots \ldots \ldots \ldots \ldots$

catenulatus Oliv., $1807 \ldots \ldots \ldots \ldots \ldots \ldots \ldots$

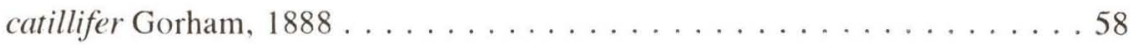

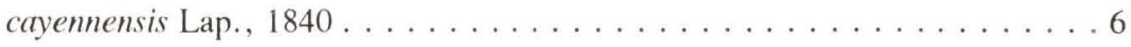

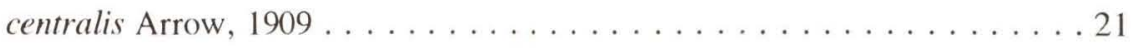

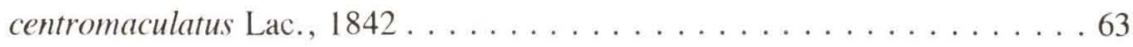

centumpunctatus Herbst, $1783 \ldots \ldots \ldots \ldots \ldots \ldots$

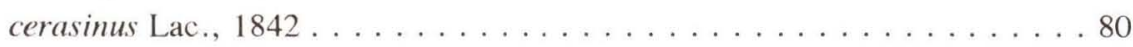

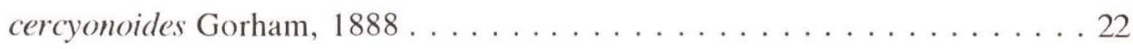

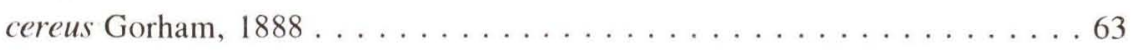

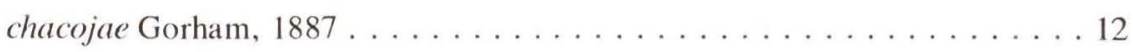




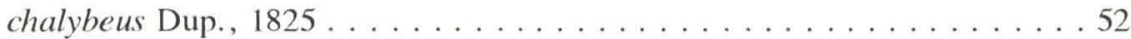

chamoeleo Lac., $1842 \ldots \ldots \ldots \ldots \ldots \ldots \ldots \ldots \ldots \ldots \ldots$

championi Gorham, $1888 \ldots \ldots \ldots \ldots \ldots \ldots \ldots \ldots \ldots \ldots \ldots \ldots \ldots$

chaparensis Delk., $1957 \ldots \ldots \ldots \ldots \ldots \ldots$

Charidemus Gistel, $1848 \ldots \ldots \ldots \ldots \ldots \ldots \ldots \ldots \ldots$

chelonarius Lac., $1842 \ldots \ldots$. . . . . . . . . . . . . . . . . 69

chevrolati Lac., $1842 \ldots \ldots \ldots$. . . . . . . . . . . . . . . 89

chevrolati Crotch, $1876 \ldots \ldots \ldots \ldots \ldots \ldots \ldots$

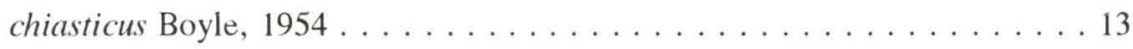

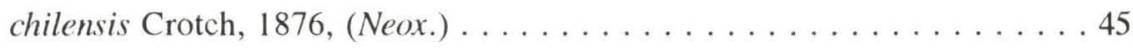

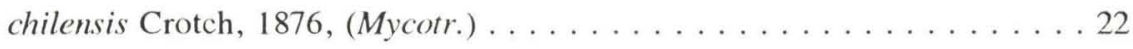

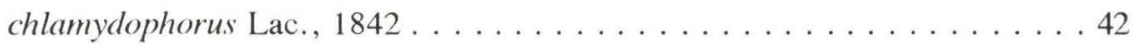

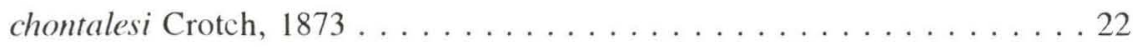

chrysomelinus Lac., $1842 \ldots \ldots \ldots \ldots \ldots \ldots \ldots \ldots \ldots$

cinctellus Lac., $1842 \ldots \ldots \ldots \ldots \ldots \ldots \ldots \ldots \ldots \ldots \ldots$

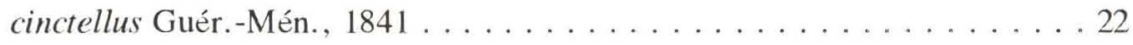

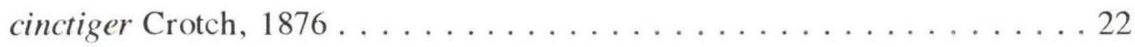

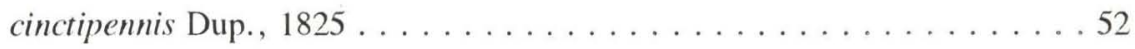

cinctus Herbst, $1799 \ldots \ldots \ldots \ldots . \ldots \ldots \ldots$

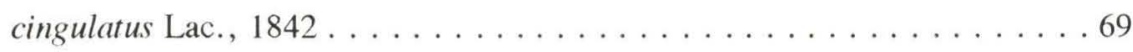

cingulatus Crotch, $1876 \ldots \ldots \ldots \ldots \ldots \ldots$

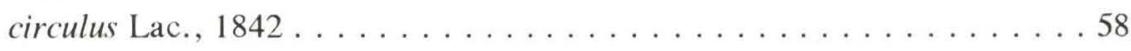

circumcinctus Delk., $1957 \ldots \ldots \ldots \ldots \ldots \ldots \ldots$

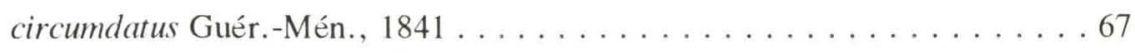

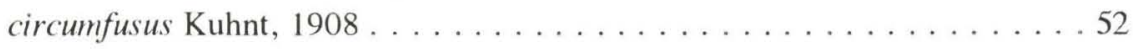

circumscriptus Dup., $1825 \ldots \ldots \ldots \ldots \ldots \ldots \ldots$

claropictus Kuhnt, $1910 \ldots \ldots \ldots \ldots \ldots \ldots$

clarosignatus Kuhnt, $1910 \ldots \ldots \ldots \ldots \ldots \ldots \ldots \ldots \ldots$

clarosignatus Kuhnt, $1908 \ldots \ldots \ldots \ldots$. . . . . . . . . . . . . 89

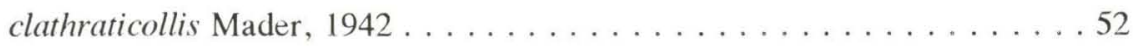

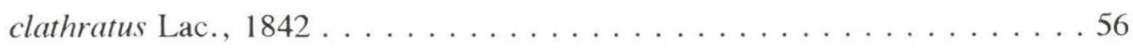

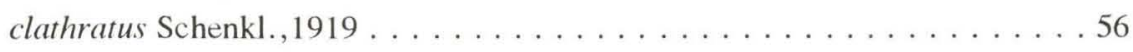

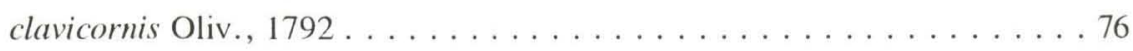

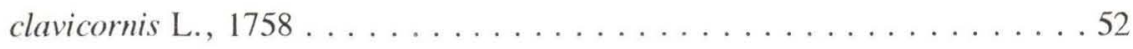

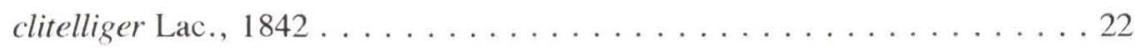

coadunatus Lac., $1842 \ldots \ldots \ldots \ldots \ldots \ldots \ldots \ldots \ldots \ldots$

Revta bras. Zool. $11(1): 1-175,1994$ 


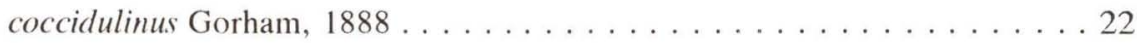

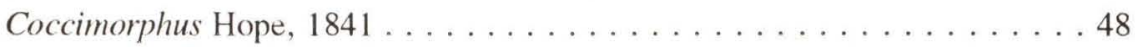

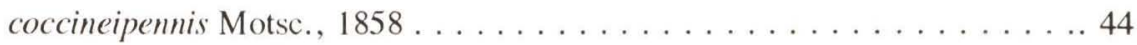

coccinellinus Crotch, $1876 \ldots \ldots \ldots \ldots \ldots \ldots \ldots$

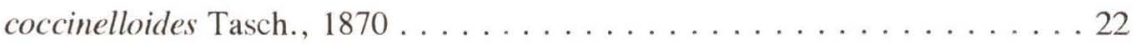

coccinelloides Dup., 1825 . . . . . . . . . . . . . . . . . . . . . 49

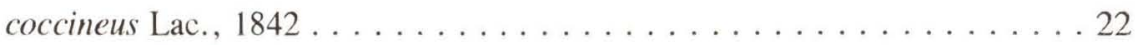

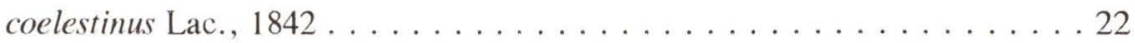

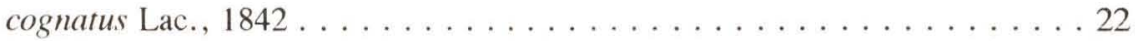

collaris Deelder, $1942 \ldots \ldots \ldots \ldots \ldots \ldots \ldots \ldots \ldots$

collaris Kuhnt, $1908 \ldots \ldots \ldots \ldots \ldots \ldots \ldots$

collatinus Crotch, $1876 \ldots \ldots \ldots \ldots \ldots \ldots \ldots \ldots \ldots$

colombi Guér.-Mén., $1844 \ldots \ldots \ldots$. . . . . . . . . . . . . . 68

coloradana Casey, $1924 \ldots \ldots \ldots \ldots \ldots$. . . . . . . . . . . 40

columbiae Kuhnt, $1908 \ldots \ldots \ldots \ldots \ldots 1$

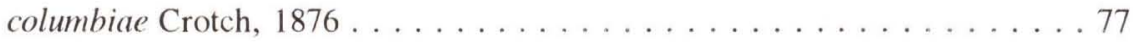

columbianus Lac., $1842 \ldots \ldots \ldots \ldots \ldots \ldots$

completus Kuhnt, $1908 \ldots \ldots \ldots \ldots \ldots \ldots \ldots$. . . . . . . . . 97

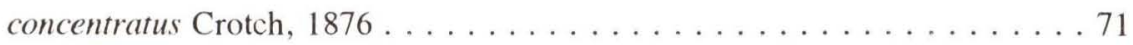

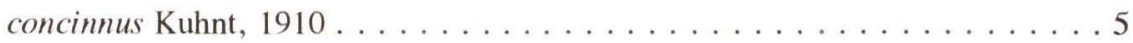

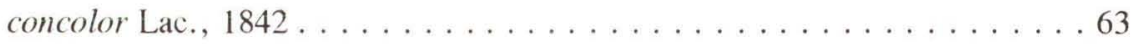

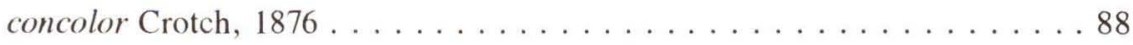

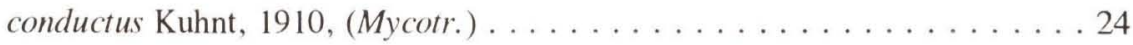

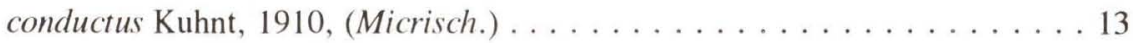

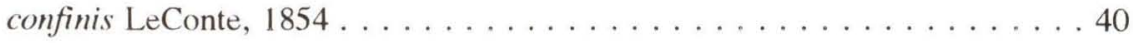

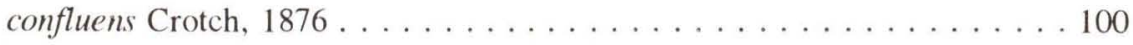

conformis Lac., $1842 \ldots \ldots \ldots \ldots \ldots \ldots$

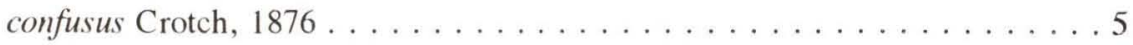

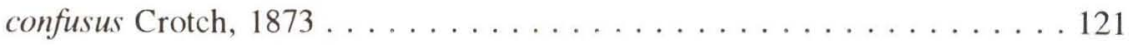

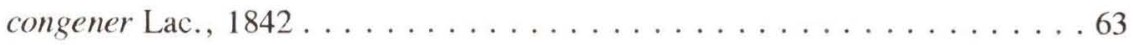

conjugens Kuhnt, $1908 \ldots \ldots \ldots \ldots \ldots \ldots \ldots \ldots$

conjunctus Crotch, $1876 \ldots \ldots \ldots \ldots \ldots \ldots \ldots \ldots \ldots$

connectens Crotch, $1876 \ldots \ldots \ldots \ldots \ldots$

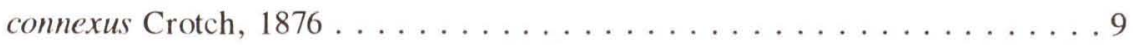

consanguineus Lac., $1842 \ldots \ldots \ldots \ldots \ldots \ldots \ldots$

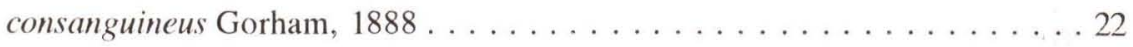




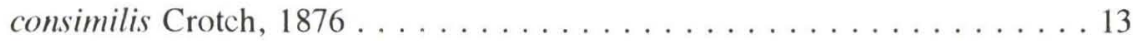

consociatus Kunht, 1910 . . . . . . . . . . . . . . . . . . . . . . 24

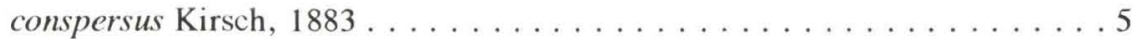

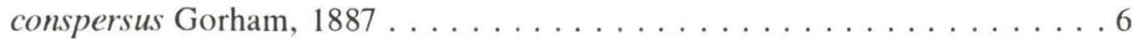

conspersus Dup., 1825 . . . . . . . . . . . . . . . . . . . . . 69

conspersus Germ., $1824 \ldots \ldots \ldots \ldots \ldots \ldots$. . . . . . . . . . 37

conspicillatus Gorham, $1888 \ldots \ldots \ldots \ldots \ldots \ldots \ldots \ldots \ldots \ldots \ldots$

consularis Guér.-Mén., $1855 \ldots \ldots \ldots \ldots \ldots \ldots \ldots$

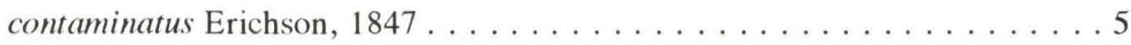

contractus Kuhnt, $1908 \ldots \ldots \ldots$. . . . . . . . . . . . . . . 89

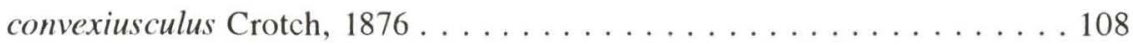

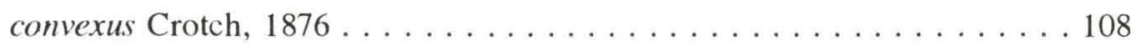

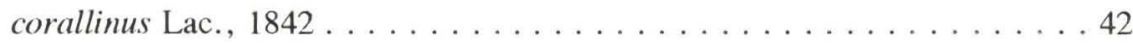

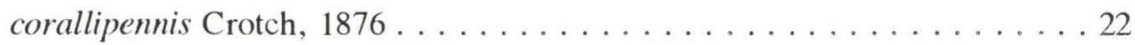

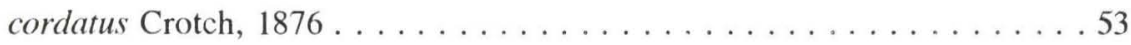

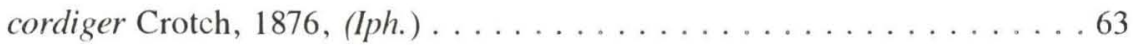

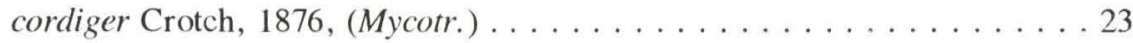

cornaliae Guér.-Mén., 1855 . . . . . . . . . . . . . . . . . . . . 89

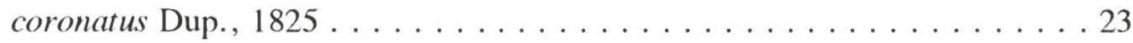

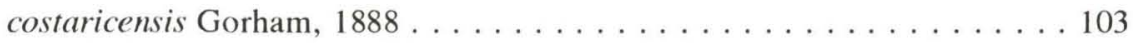

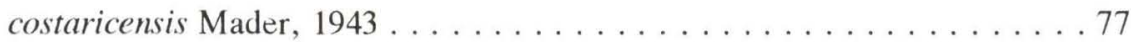

crabronoides Lac., $1842 \ldots \ldots \ldots \ldots$. . . . . . . . . . . . . . . 113

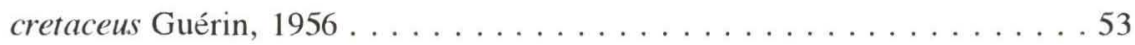

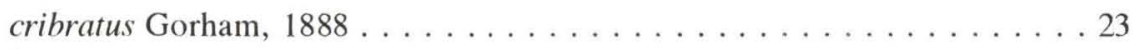

cribripennis Achard, $1926 \ldots \ldots \ldots \ldots \ldots \ldots$

cribrosus Lac., $1842 \ldots \ldots \ldots \ldots \ldots \ldots \ldots \ldots \ldots$

crocicollis Lac., $1842 \ldots \ldots \ldots \ldots . \ldots \ldots \ldots$

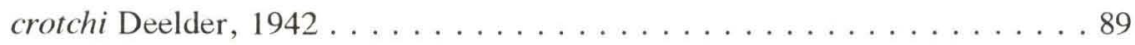

cruciatus Lac., $1842 \ldots \ldots \ldots \ldots \ldots \ldots$. . . . . . . . . . . . . 87

crucifer Kuhnt, $1908 \ldots \ldots \ldots \ldots$. . . . . . . . . . . . . . . . 89

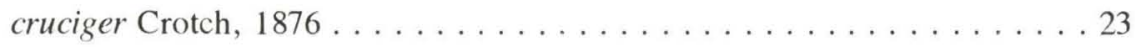

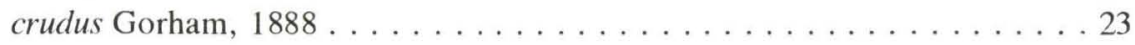

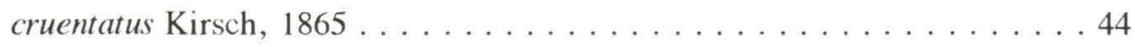

cruentipennis Lac., $1842 \ldots \ldots \ldots \ldots$. . . . . . . . . . . . 39

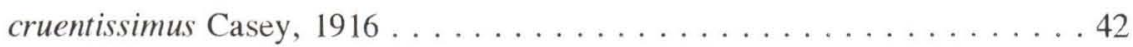

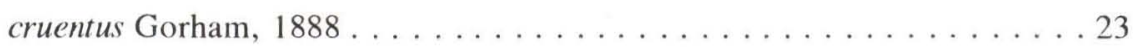

Revta bras. Zool. $11(1): 1$ - 175, 1994 


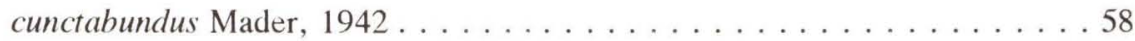

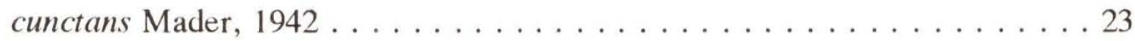

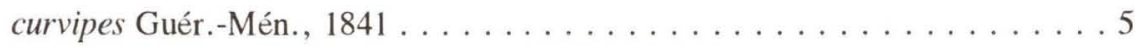

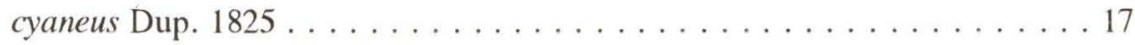

cyanipennis Guér.-Mén., $1841 \ldots \ldots \ldots \ldots \ldots \ldots \ldots \ldots \ldots \ldots \ldots$

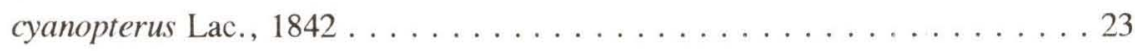

cyanopterus Erichson, $1847 \ldots \ldots \ldots$

Cyclomorphus Hope, $1841 \ldots \ldots \ldots \ldots \ldots \ldots$

Cypherotylus Crotch, $1873 \ldots \ldots \ldots \ldots 101$

Cyrtomorphus Lac., $1842 \ldots \ldots \ldots \ldots \ldots$

Cyrtotriplax Crotch, $1873 \ldots \ldots \ldots \ldots$

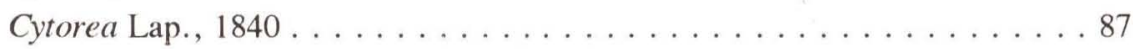

d'orbignyi Guér.-Mén., $1841 \ldots \ldots \ldots \ldots$. . . . . . . . . . . 121

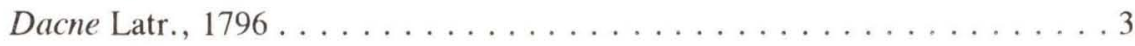

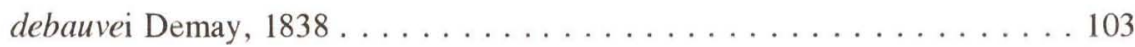

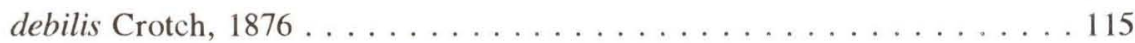

decemmaculatus Dup., 1825 . . . . . . . . . . . . . . . . . . 109

decemnotatus Dup., $1825 \ldots \ldots \ldots \ldots$. . . . . . . . . . . 69

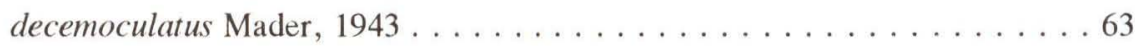

decemplagiatus Kuhnt, $1910 \ldots \ldots \ldots \ldots \ldots \ldots \ldots \ldots \ldots \ldots \ldots \ldots \ldots$

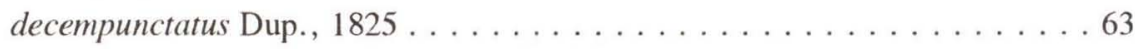

decempunctatus Guér.-Mén., $1841 \ldots \ldots \ldots \ldots \ldots$

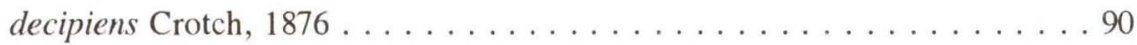

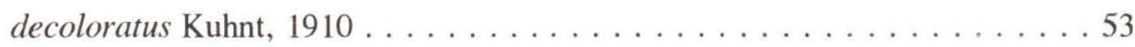

decoloratus Crotch, $1876 \ldots \ldots \ldots \ldots \ldots \ldots \ldots \ldots \ldots \ldots \ldots \ldots$

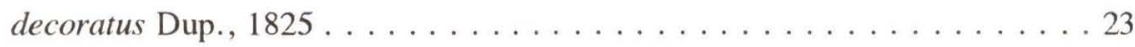

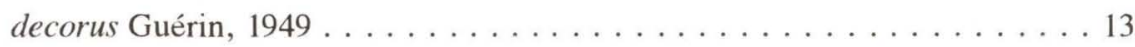

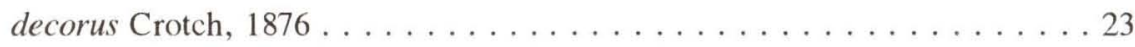

decussatus Lac., $1842 \ldots \ldots \ldots \ldots \ldots \ldots \ldots \ldots \ldots \ldots \ldots$

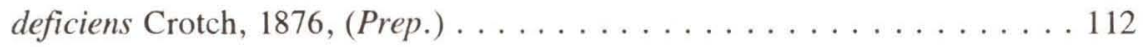

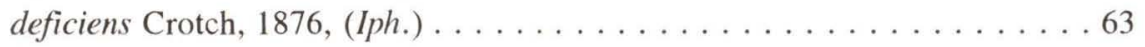

dejeani Lac., $1842 \ldots \ldots \ldots \ldots \ldots \ldots \ldots \ldots \ldots \ldots \ldots \ldots \ldots$

delenitus Alv., $1976 \ldots \ldots \ldots \ldots \ldots \ldots$

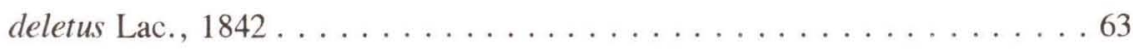

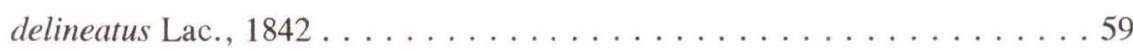

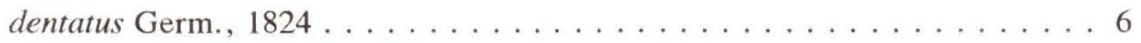




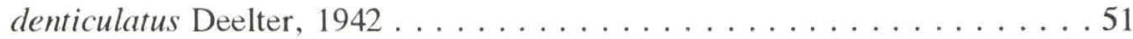

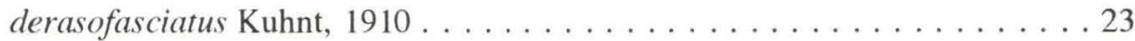

detrahens Crotch, $1876 \ldots \ldots \ldots \ldots \ldots \ldots \ldots$

detritus Lac., $1842 \ldots \ldots \ldots \ldots \ldots \ldots$. . . . . . . . . . . . . . . 63

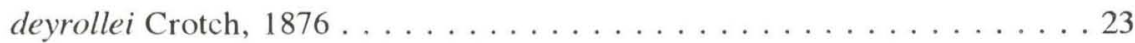

Dichomorpha Kuhnt, 1909 . . . . . . . . . . . . . . . . . . . . . . 88

dichromostigma Guér.-Mén., $1841 \ldots \ldots \ldots \ldots$. . . . . . . . . 98

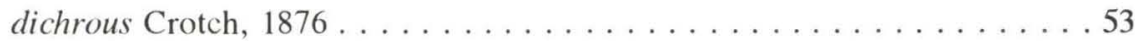

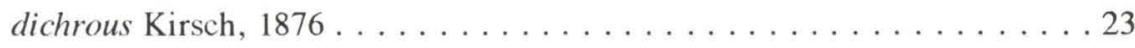

dichrous Lac., $1842 \ldots \ldots \ldots \ldots \ldots \ldots$. . . . . . . . . . . . 49

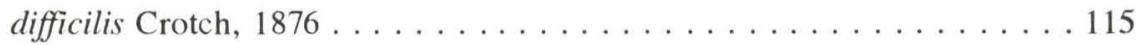

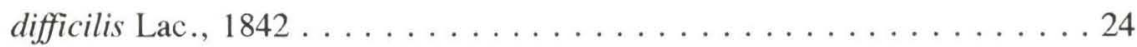

diffinis Erichson, $1847 \ldots \ldots \ldots \ldots \ldots \ldots \ldots \ldots \ldots \ldots \ldots \ldots \ldots$

dilaceratus Kirsch, $1876 \ldots \ldots \ldots \ldots \ldots \ldots$

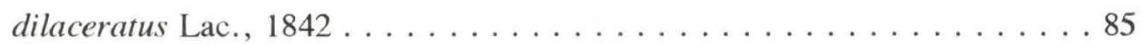

dilectus Gorham, $1888 \ldots \ldots \ldots \ldots \ldots \ldots \ldots$

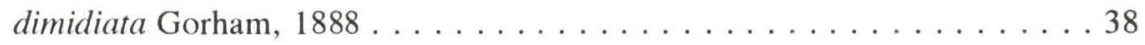

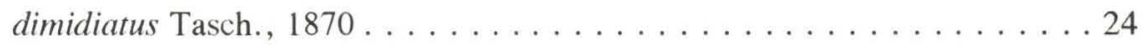

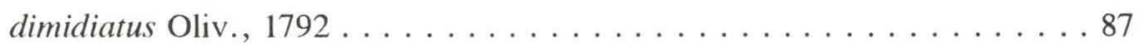

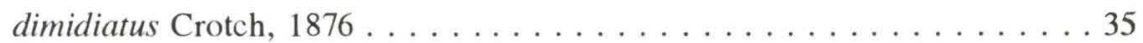

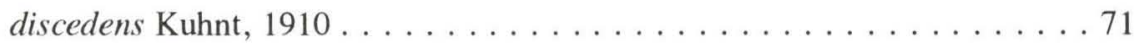

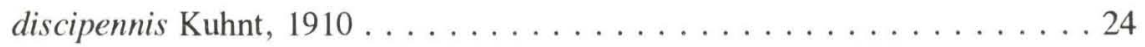

discipennis Lac., $1842 \ldots \ldots \ldots \ldots \ldots \ldots \ldots \ldots$

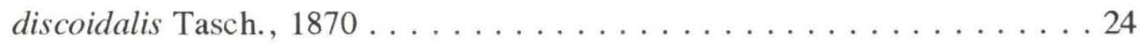

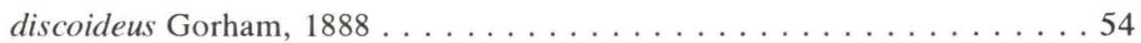

discoideus Oliv., 1807 . . . . . . . . . . . . . . . . . . . . . . . . 108

disconigrum Mader, 1942, (Micrisch.) . . . . . . . . . . . . . 13

disconigrum Mader, 1942, (Iph.) . . . . . . . . . . . . . . . . 77

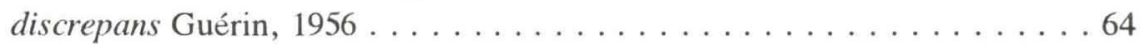

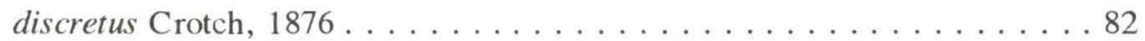

discus Lac., $1842 \ldots \ldots \ldots \ldots$. . . . . . . . . . . . . . . . . . . 64

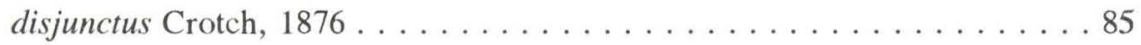

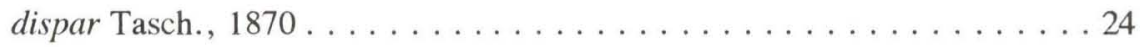

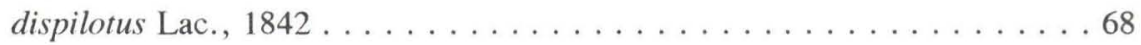

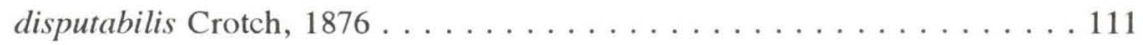

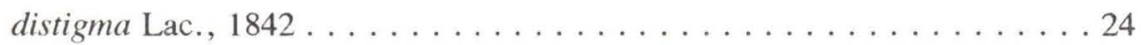




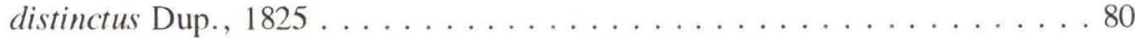

distinguendus Lac., $1842 \ldots \ldots \ldots \ldots \ldots \ldots$

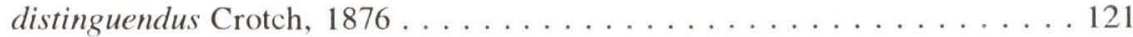

distinguendus Arrow, $1909 \ldots \ldots \ldots \ldots$

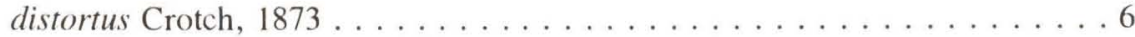

divisa Gorham, $1888 \ldots \ldots \ldots \ldots \ldots$. . . . . . . . . . . . . 40

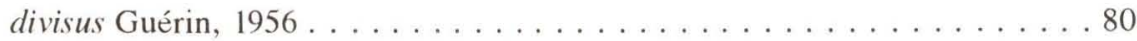

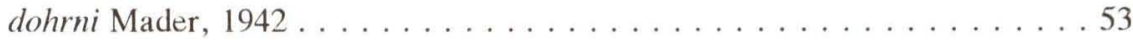

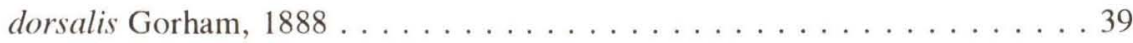

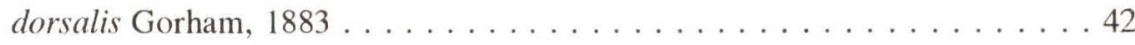

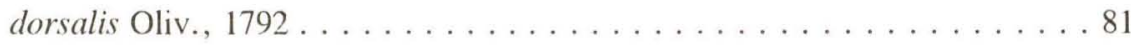

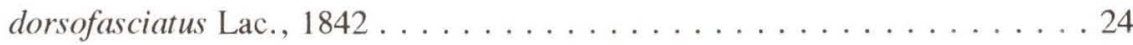

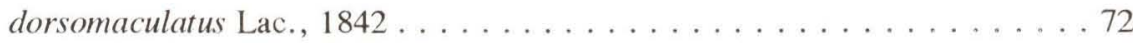

dorsonotatus Lac., 1842, (Mycotr.) . . . . . . . . . . . . . . 24

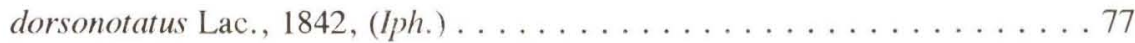

dromedarius Lac., $1842 \ldots \ldots \ldots \ldots$. . . . . . . . . . . . . . 103

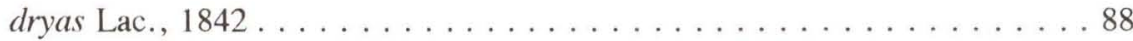

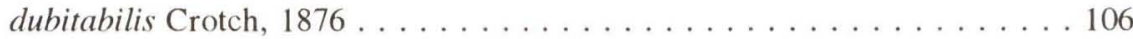

dubius Gorham, $1888 \ldots \ldots \ldots \ldots \ldots \ldots$

dubius Lac., 1842. . . . . . . . . . . . . . . . . . . . . . . . . 24

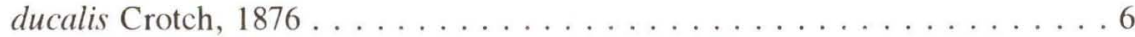

dunedinensis Leng, $1920 \ldots \ldots \ldots \ldots \ldots$

duodecimguttatus Dup., $1825 \ldots \ldots \ldots \ldots \ldots \ldots$

duodecimmaculatus Kuhnt, 1910 . . . . . . . . . . . . . . 108

duodecimpustulatus Lac., 1842 . . . . . . . . . . . . . . . . . . . . . . 59

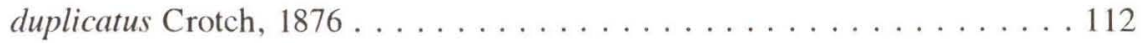

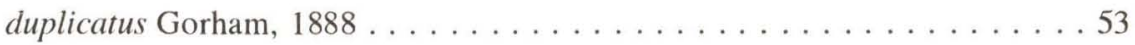

duplicatus Lac., $1842 \ldots \ldots \ldots \ldots \ldots \ldots$

duponcheli Chevr., $1834 \ldots \ldots \ldots \ldots \ldots \ldots \ldots \ldots \ldots \ldots \ldots$

duponcheli Lac., $1842 \ldots \ldots \ldots \ldots$. . . . . . . . . . . . . . . 121

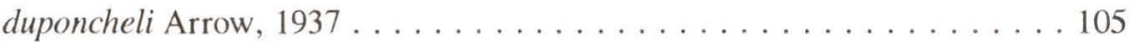

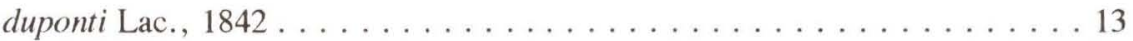

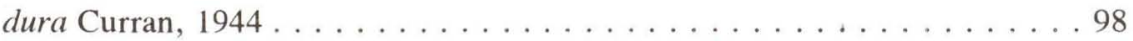

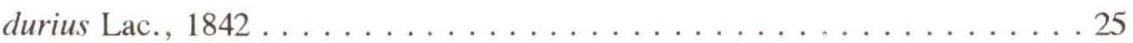

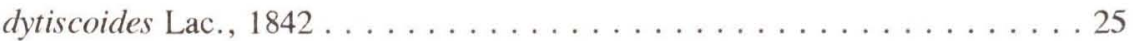

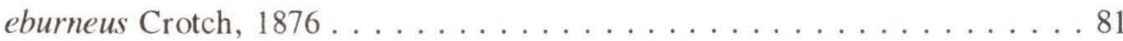


ecuadorensis Crotch, $1876 \ldots \ldots \ldots \ldots \ldots$

ecuadorica Crotch, $1876 \ldots \ldots \ldots \ldots \ldots$

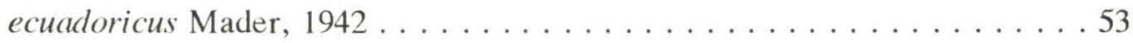

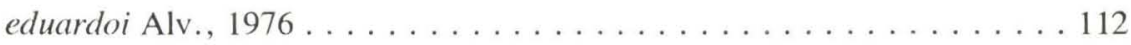

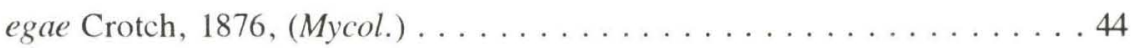

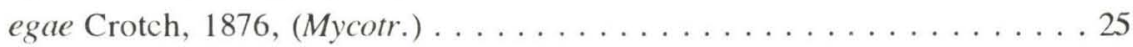

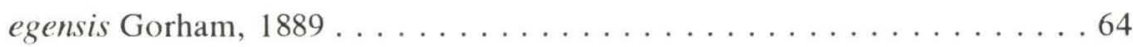

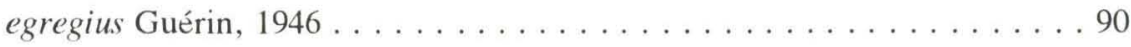

elateroides Gorham, $1888 \ldots \ldots \ldots \ldots$. . . . . . . . . . . . 39

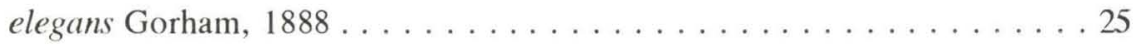

elegans Guér.-Mén., $1841 \ldots \ldots \ldots \ldots$

elegans Mader, 1942, (Neopr.) . . . . . . . . . . . . . . . 115

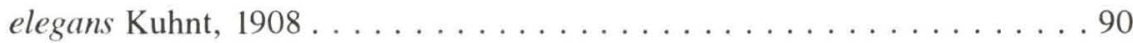

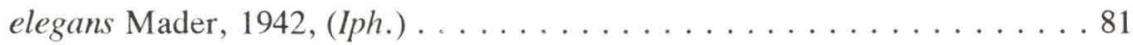

elegantulus Guérin, $1946 \ldots \ldots \ldots \ldots \ldots$

elegantulus Lac., $1842 \ldots \ldots \ldots \ldots \ldots \ldots$

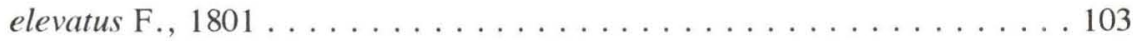

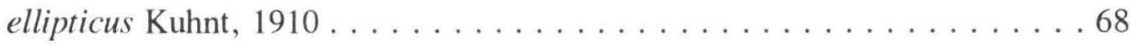

Ellipticus Chevr., $1837 \ldots \ldots \ldots \ldots \ldots$

Ellipticus (s.str.) Chevr., $1837 \ldots \ldots \ldots \ldots$

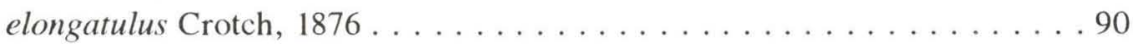

elongatus Gorham, $1883 \ldots \ldots \ldots \ldots \ldots \ldots \ldots \ldots \ldots \ldots \ldots \ldots \ldots$

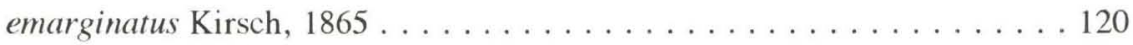

emys Lac., $1842 \ldots \ldots \ldots \ldots$. . . . . . . . . . . . . . . . . . 49

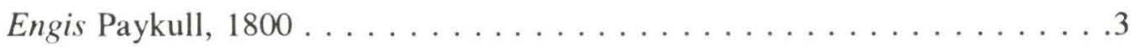

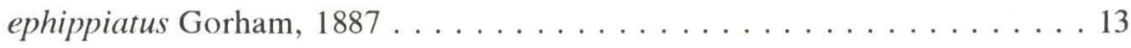

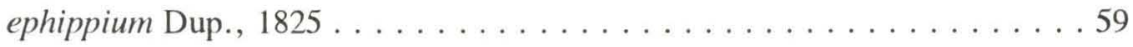

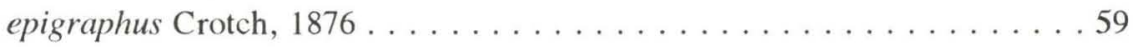

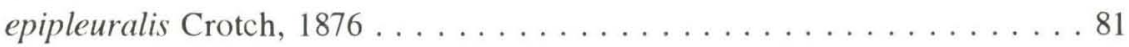

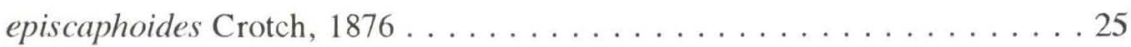

episcaphulinus Gorham, $1887 \ldots \ldots \ldots \ldots \ldots \ldots \ldots \ldots \ldots \ldots \ldots$

episcopalis Lac., 1842. . . . . . . . . . . . . . . . . . 25

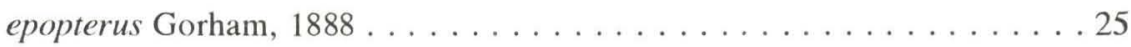

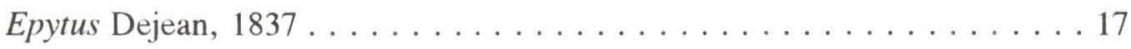

equestris Lac., $1842 \ldots \ldots \ldots \ldots \ldots \ldots \ldots \ldots \ldots \ldots \ldots \ldots$

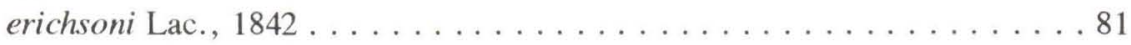


Erotylina Curran, $1944 \ldots \ldots \ldots \ldots \ldots \ldots$

erotyloides Crotch, $1876 \ldots \ldots \ldots \ldots \ldots \ldots \ldots$

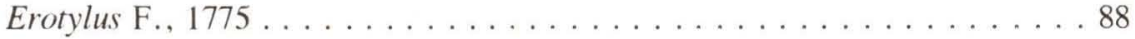

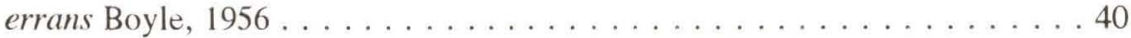

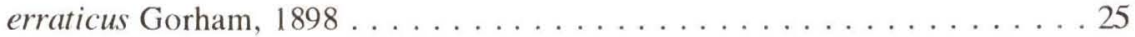

erythrocephalus Oliv., $1807 \ldots \ldots \ldots \ldots \ldots \ldots \ldots$

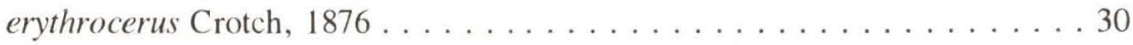

erythrogonus Crotch, $1876 \ldots \ldots \ldots \ldots \ldots$

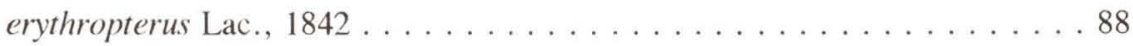

Euphanistes Lac., $1842 \ldots \ldots \ldots \ldots \ldots$. . . . . . . . . . . 46

Eurycardius Lac., $1842 \ldots \ldots \ldots \ldots \ldots \ldots$

exiguenotatus Gorham, $1888 \ldots \ldots \ldots \ldots \ldots \ldots$

eximius Lac., $1842 \ldots \ldots \ldots \ldots \ldots$. . . . . . . . . . . . . . . 59

exoticus Voet, $1778 \ldots \ldots \ldots \ldots \ldots$. . . . . . . . . . . . . . . . .

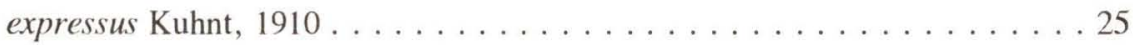

extricatus Crotch, $1876 \ldots \ldots \ldots \ldots$. . . . . . . . . . . . . 47

faba Lac., $1842 \ldots \ldots \ldots \ldots \ldots \ldots$. . . . . . . . . . . . . . . 42

fallax Guér.-Mén., $1841 \ldots \ldots \ldots \ldots \ldots$

fasciatopunctatus Lac., $1842 \ldots \ldots \ldots$. . . . . . . . . . 80

fasciatus Oliv., $1792 \ldots \ldots \ldots \ldots \ldots$. . . . . . . . . . . 86

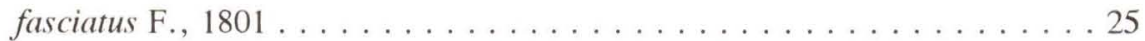

fasciellus Crotch, $1876 \ldots \ldots \ldots \ldots \ldots$

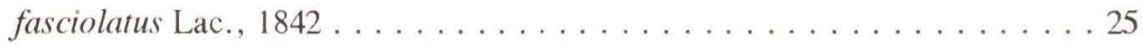

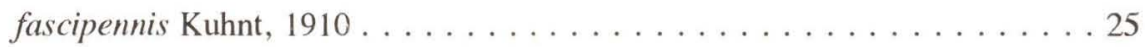

fasciunculus Crotch, $1876 \ldots \ldots \ldots \ldots \ldots$

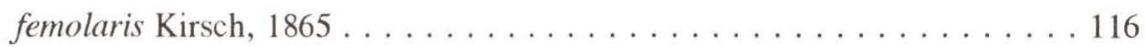

femoralis Chevr., $1841 \ldots \ldots \ldots \ldots \ldots \ldots \ldots \ldots \ldots \ldots \ldots$

fenestratus Gorham, $1888 \ldots \ldots \ldots \ldots$. . . . . . . . . . . 103

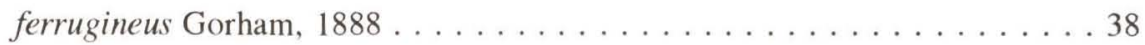

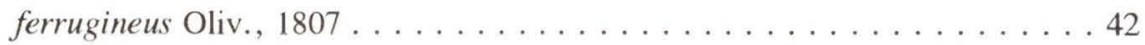

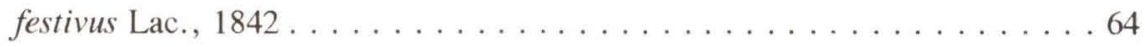

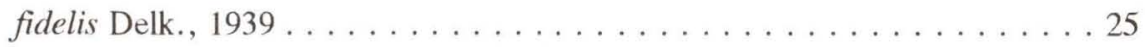

figuratus Lac., $1842 \ldots \ldots \ldots . \ldots \ldots$

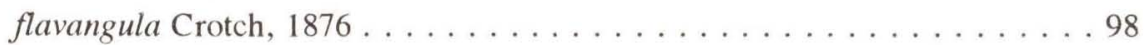

flavicollis Lac., $1842 \ldots \ldots \ldots \ldots$. . . . . . . . . . . . . 40

flavipennis Guér.-Mén., $1844 \ldots \ldots \ldots \ldots$. . . . . . . . . . . 45 


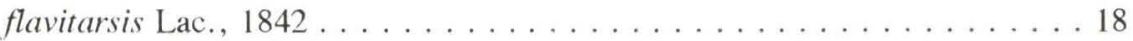

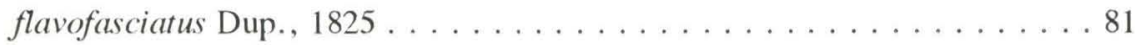

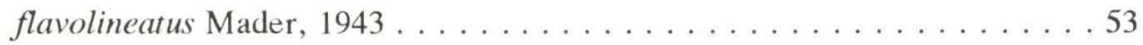

flavomaculatus Mader, $1943 \ldots \ldots \ldots \ldots$

flavomarginatus Lac., $1842 \ldots \ldots \ldots \ldots$. . . . . . . . . . 26

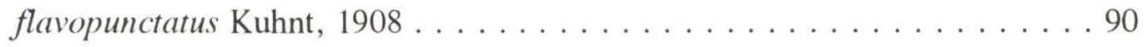

flavosignatus Dup., $1825 \ldots \ldots \ldots \ldots$. . . . . . . . . . . . . 81

flavotaeniatus Kuhnt, $1908 \ldots \ldots \ldots \ldots \ldots \ldots$

flavovittatoides Mader, $1943 \ldots \ldots$. . . . . . . . . . . . . 69

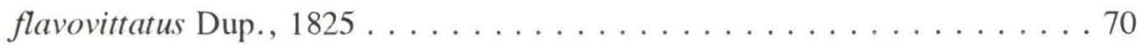

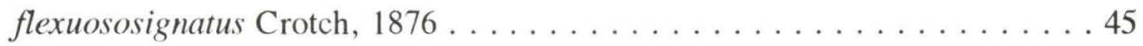

flexuosus Lac., 1842 . . . . . . . . . . . . . . . . . . . . . . 70

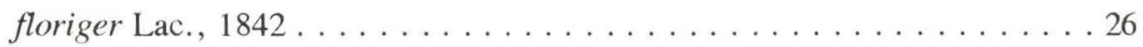

floripesae Alv., $1962 \ldots \ldots \ldots$. . . . . . . . . . . . . . . . . 49

foraminosus Lac., $1842 \ldots \ldots \ldots$. . . . . . . . . . . . . . . 101

forsteri Delk., 1957 . . . . . . . . . . . . . . . . . . . . . . 90

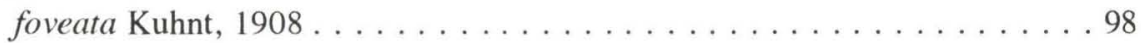

foveicollis Lac., $1842 \ldots \ldots \ldots \ldots$. . . . . . . . . . . . . . . . . 49

fractus Crotch, $1876 \ldots \ldots \ldots \ldots \ldots$

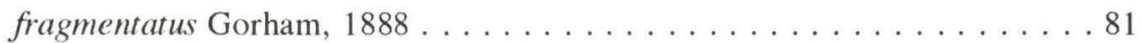

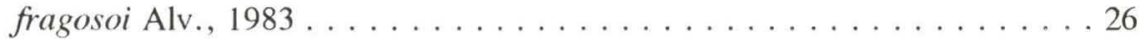

fraternus Lac., $1842 \ldots \ldots \ldots \ldots \ldots \ldots \ldots \ldots \ldots$

frenatus Guér.-Mén., 1841 . . . . . . . . . . . . . . . . . . . . 49

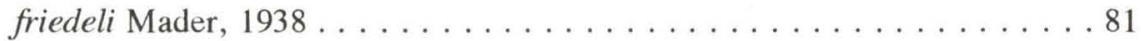

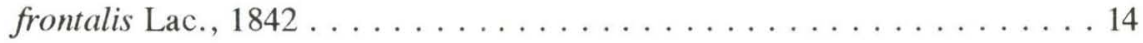

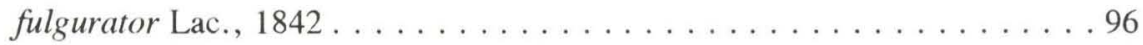

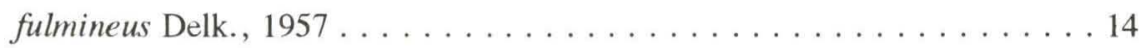

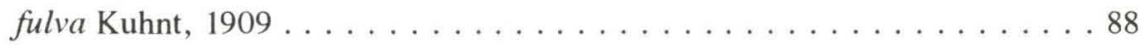

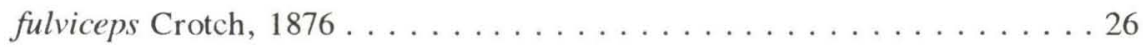

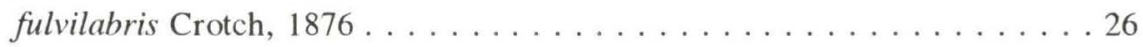

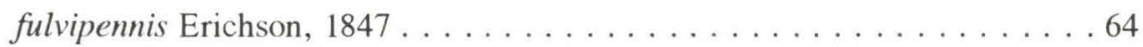

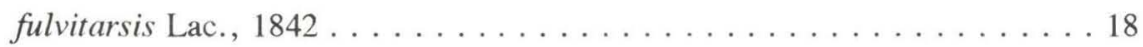

fulviventris Gorham, $1888 \ldots \ldots \ldots \ldots \ldots \ldots \ldots 77$

fulvofasciatus Kuhnt, $1908 \ldots \ldots \ldots$. . . . . . . . . . . . 90

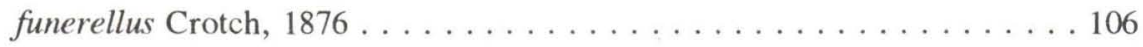

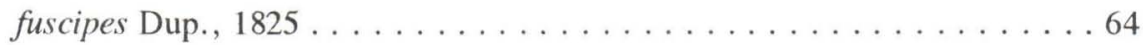




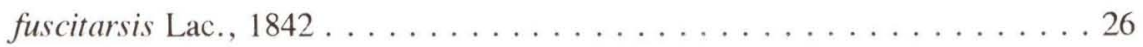

fuscomaculatus Dup., $1825 \ldots \ldots \ldots$. . . . . . . . . . . . . 64

gaumeri Gorham, $1888 \ldots \ldots \ldots$. . . . . . . . . . . . . . 103

Gemellatotelus Mader, $1942 \ldots \ldots \ldots \ldots \ldots \ldots$

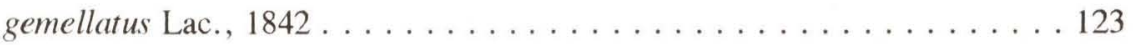

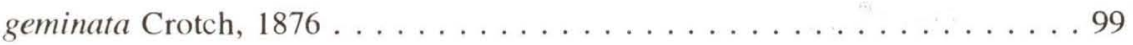

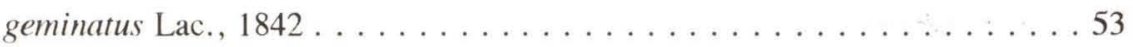

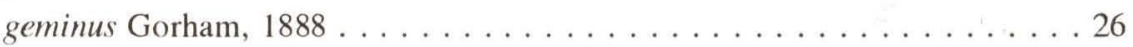

gemmata $\mathrm{F} ., 1792 \ldots \ldots \ldots \ldots \ldots \ldots \ldots$

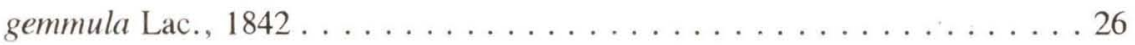

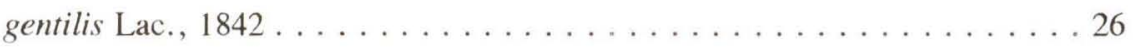

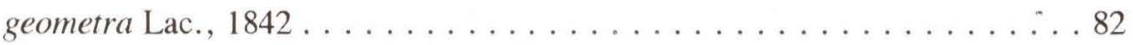

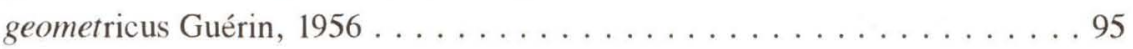

germari Lac., $1842 \ldots \ldots \ldots$. . . . . . . . . . . . . . . . . . . 87

ghiliani Guér.-Mén., 1855 . . . . . . . . . . . . . . . . . . . . . . 99

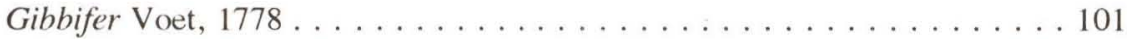

gibbosus L., $1763 \ldots \ldots \ldots \ldots \ldots \ldots \ldots$. . . . . . . . . . . . . 104

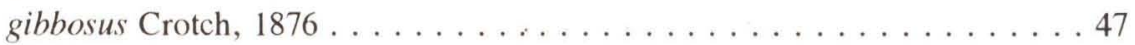

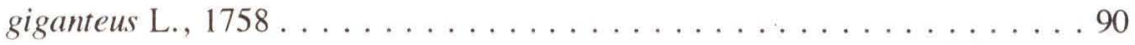

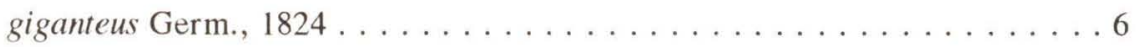

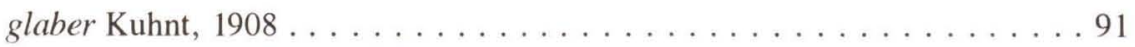

glaber Gorham, $1888 \ldots \ldots \ldots \ldots \ldots \ldots \ldots \ldots \ldots \ldots \ldots \ldots$

Glabratotelus Mader, 1942 . . . . . . . . . . . . . . . . . . . . . 123

glabratus Waterh., $1879 \ldots \ldots \ldots \ldots \ldots$. . . . . . . . . . . . 47

globosus Guér.-Mén., 1841 . . . . . . . . . . . . . . . . . . 47

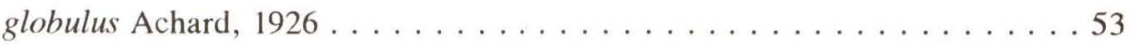

glyptoderus Lac., $1842 \ldots \ldots \ldots \ldots \ldots \ldots \ldots \ldots \ldots$

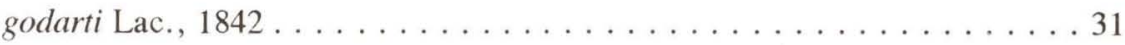

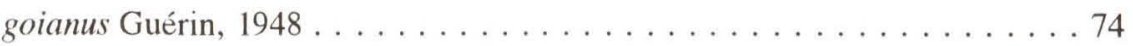

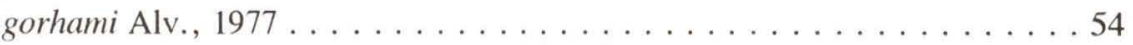

gorhami Mader, $1942 \ldots \ldots \ldots \ldots \ldots$. . . . . . . . . . . . 9

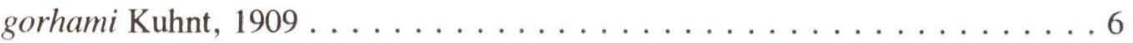

goryi Guér.-Mén., 1841 . . . . . . . . . . . . . . . . . . . . . . . . . 104

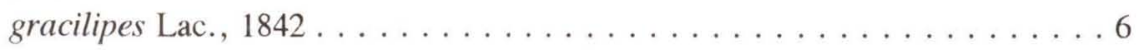

gracilis Kuhnt, $1908 \ldots \ldots \ldots \ldots \ldots$. . . . . . . . . . . . . . 104

grammicoides Mader, $1943 \ldots \ldots \ldots \ldots$. . . . . . . . . . . . 64 


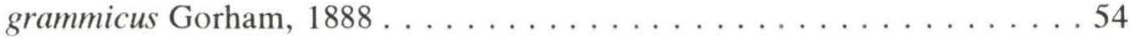

grammicus Gorham, $1883 \ldots \ldots \ldots \ldots \ldots \ldots$

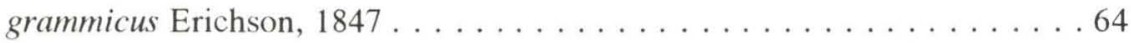

grammistes Lac., $1842 \ldots \ldots \ldots \ldots \ldots$

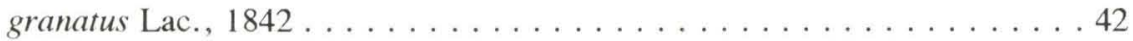

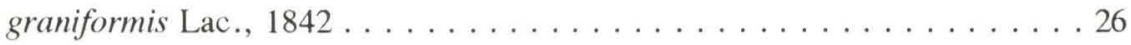

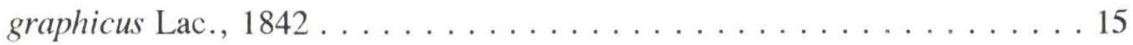

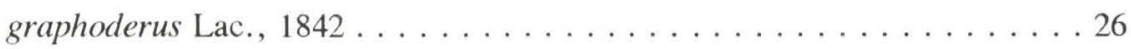

gratiosus Guér.-Mén., $1844 \ldots \ldots \ldots \ldots \ldots \ldots$

gronovii Herbst, $1783 \ldots \ldots \ldots$. . . . . . . . . . . . . . . . 107

guadeloupensis $\mathrm{F} ., 1792 \ldots \ldots \ldots \ldots \ldots 73$

guatemalae Crotch, 1876, (Isch.) . . . . . . . . . . . . . . 10

guatemalae Crotch, 1876, (Gibb.) . . . . . . . . . . . . . . . . . 104

guatemalae Crotch, 1876, (Olig.) . . . . . . . . . . . . . . . . 110

guatemalae Crotch, 1876, (Mycotr.) . . . . . . . . . . . . . . 27

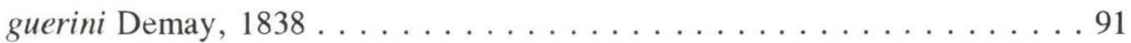

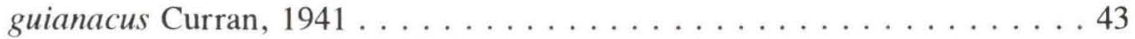

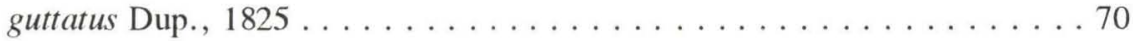

guttiger Kuhnt, $1910 \ldots \ldots \ldots \ldots \ldots \ldots \ldots$

habrodactyloides Crotch, $1876 \ldots \ldots \ldots \ldots \ldots \ldots$

Habrodactylus Lac., 1842 . . . . . . . . . . . . . . . . . . . . . . 62

haemapterus Gorham, $1888 \ldots \ldots \ldots \ldots$. . . . . . . . . . 27

haematicus Gorham., 1888 . . . . . . . . . . . . . . . . . . . . . 27

haematites Lac., 1842 . . . . . . . . . . . . . . . . . . . . . . . 64

haematocephalus Lac., $1842 \ldots \ldots \ldots \ldots \ldots$. . . . . . . . . 74

Haematochiton Gorham, 1888 . . . . . . . . . . . . . . . . . . 39

haematomelas Lac., $1842 \ldots \ldots \ldots \ldots$. . . . . . . . . . . . . 64

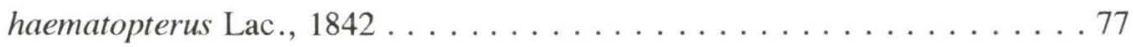

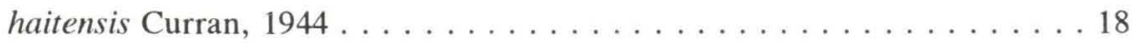

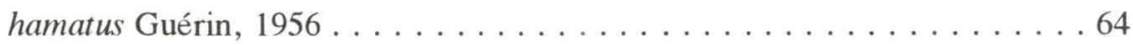

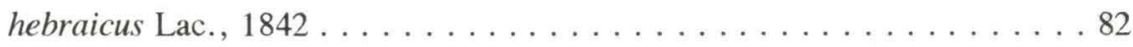

helopioides Dup., $1825 \ldots \ldots \ldots$. . . . . . . . . . . . 99

hemisphaericus Lac., $1842 \ldots \ldots \ldots \ldots$. . . . . . . . . . . 54

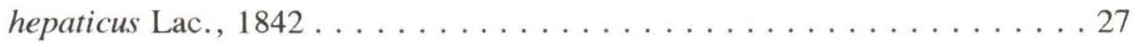

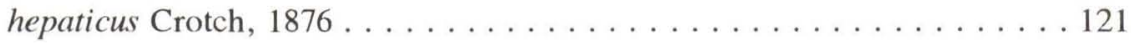

hepaticus Kirsch, $1876 \ldots \ldots \ldots \ldots \ldots \ldots$

Revta bras. Zool. 11 (1): 1 - 175, 1994 
herbsti Lac., $1842 \ldots \ldots \ldots \ldots \ldots$. . . . . . . . . . . . . . . 113

heros Guér.-Mén., $1841 \ldots \ldots \ldots \ldots \ldots \ldots \ldots \ldots$

herpestes Lac., $1842 \ldots \ldots \ldots \ldots$. . . . . . . . . . . . . 99

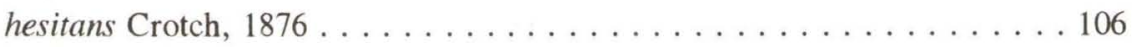

heterogrammus Lac., $1842 \ldots \ldots \ldots \ldots \ldots$. . . . . . . . . . . 107

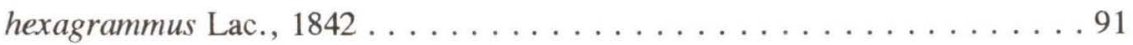

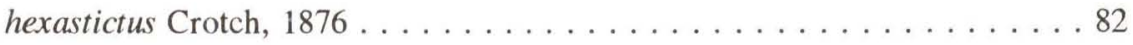

hieroglyphicus Dup., $1825 \ldots \ldots \ldots \ldots \ldots \ldots$

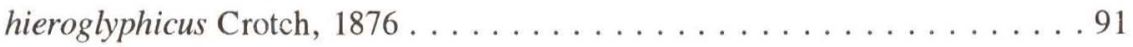

hilaris Lac., 1842 . . . . . . . . . . . . . . . . . . . . . . 27

hirudo Gorham, $1888 \ldots \ldots \ldots \ldots \ldots \ldots \ldots \ldots \ldots \ldots \ldots \ldots$

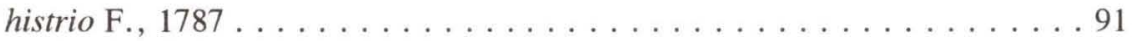

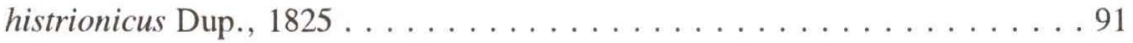

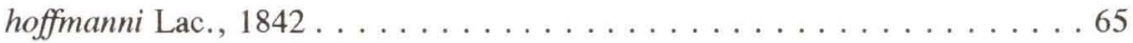

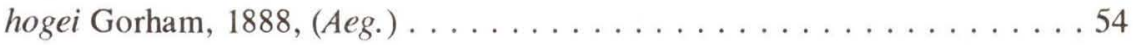

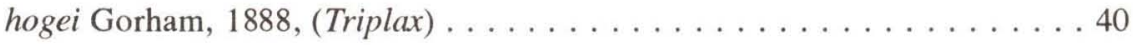

Homoeotelus Erichson, 1847 . . . . . . . . . . . . . . . . . 120

Homoeotelus Hader, 1942 . . . . . . . . . . . . . . . . . . . . 120

hopei Guér.-Mén., $1844 \ldots \ldots \ldots \ldots \ldots \ldots \ldots \ldots \ldots$

hopei Guér.-Mén., 1841 . . . . . . . . . . . . . . . . . . . . . . . . 119

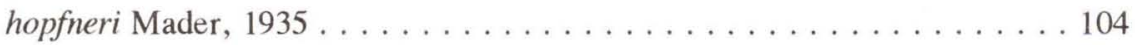

horioni Delk., $1957 \ldots \ldots \ldots \ldots \ldots \ldots \ldots \ldots \ldots \ldots$

humboldti Lac., $1842 \ldots \ldots \ldots \ldots$. . . . . . . . . . . . . 47

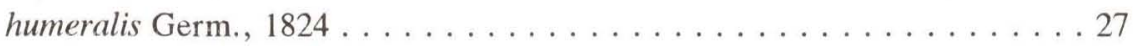

humeralis Kuhnt, $1910 \ldots \ldots \ldots \ldots \ldots \ldots \ldots \ldots \ldots \ldots \ldots \ldots \ldots$

humeralis Guérin, $1956 \ldots \ldots \ldots \ldots \ldots \ldots \ldots \ldots$

humeropictus Mader, $1943 \ldots \ldots \ldots \ldots \ldots \ldots \ldots \ldots 77$

humilis Lac., $1842 \ldots \ldots \ldots \ldots$. . . . . . . . . . . . . . . . 27

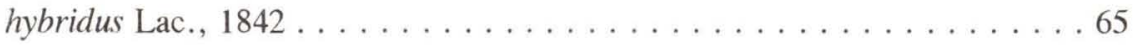

hybridus Erichson, $1847 \ldots \ldots \ldots \ldots \ldots$. . . . . . . . . . 108

hydrophiloides Lac., $1842 \ldots \ldots \ldots \ldots \ldots \ldots$

Hypselonotus, Hope, 1841 . . . . . . . . . . . . . . . . . . 101

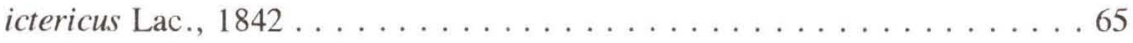

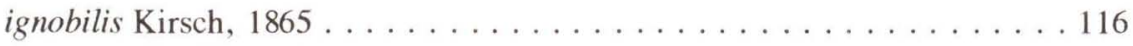

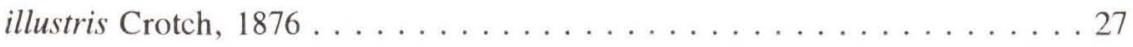

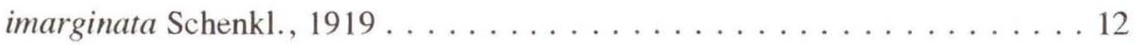




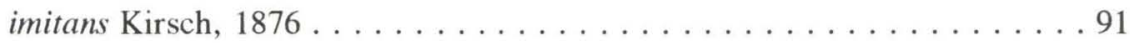

immaculatus Lac., $1842 \ldots \ldots \ldots \ldots \ldots \ldots \ldots \ldots$

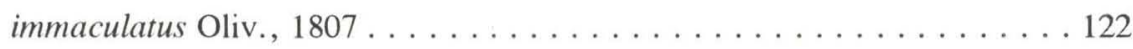

immaculatus Deelder, $1942 \ldots \ldots \ldots \ldots \ldots$

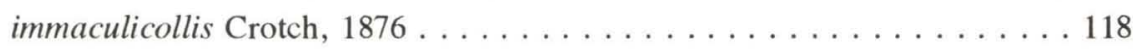

immaculipennis Mader, $1935 \ldots \ldots \ldots \ldots \ldots \ldots \ldots \ldots$

imperfecta Crotch, $1876 \ldots \ldots \ldots \ldots \ldots \ldots$

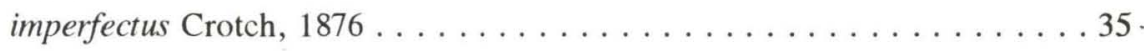

impluviatus Lac., $1842 \ldots \ldots \ldots \ldots \ldots \ldots \ldots \ldots \ldots \ldots \ldots$

impressopunctatus Crotch, $1873 \ldots \ldots \ldots \ldots \ldots \ldots \ldots$

impressopunctatus Crotch, $1876 \ldots \ldots \ldots \ldots \ldots \ldots$

impuctatus Crotch, $1876 \ldots \ldots \ldots \ldots \ldots \ldots \ldots$

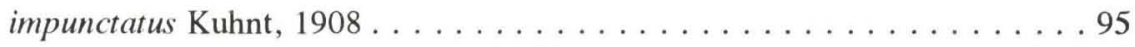

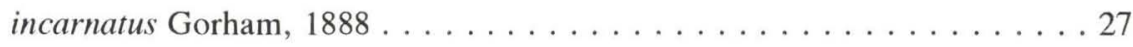

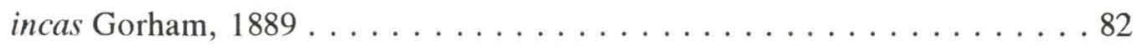

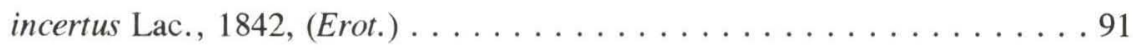

incertus Lac., 1842, (Micrisch.) . . . . . . . . . . . . . . . 14

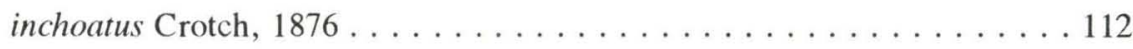

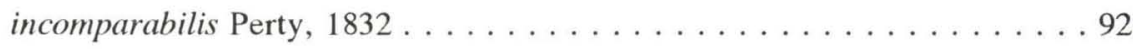

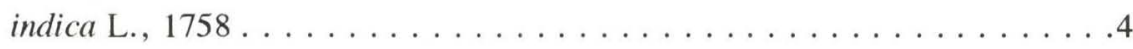

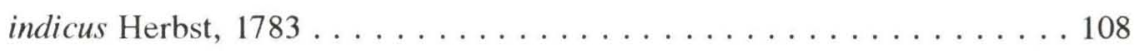

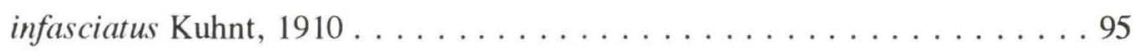

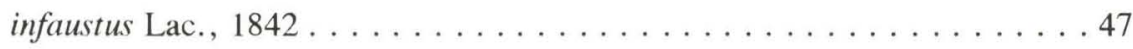

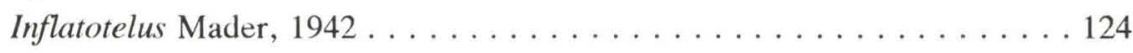

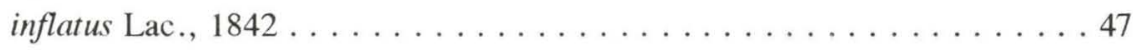

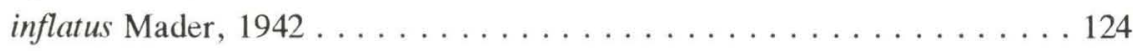

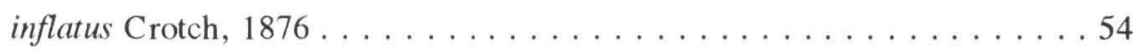

insignis Lap., $1840 \ldots \ldots \ldots \ldots$. . . . . . . . . . . . . . . . . . 19

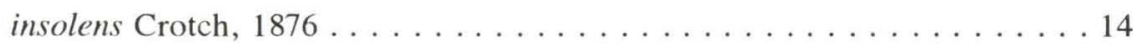

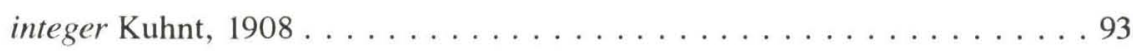

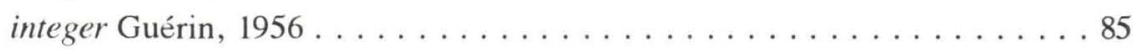

intercedens Kuhnt, $1908 \ldots \ldots \ldots \ldots$. . . . . . . . . . . . . . 104

intercedens Schenkl., 1919 . . . . . . . . . . . . . . . 74

intermedia Crotch, $1876 \ldots \ldots \ldots \ldots \ldots 9 . \ldots \ldots \ldots$

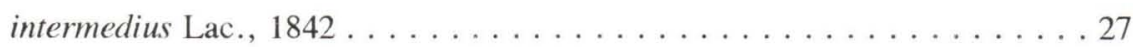

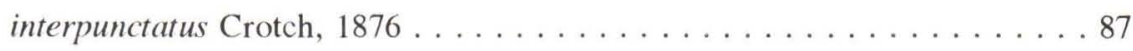

Revta bras. Zool. 11 (1): 1 - 175, 1994 
interpunctatus Gorham, $1888 \ldots \ldots \ldots \ldots \ldots \ldots \ldots \ldots \ldots$

interruptus Kuhnt, 1908 . . . . . . . . . . . . . . . . . . 74

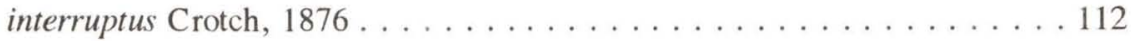

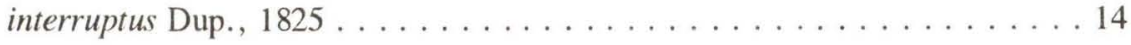

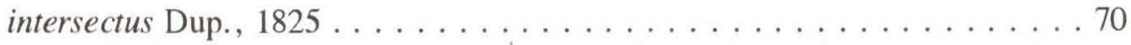

interstictus Gorham, $1888 \ldots \ldots \ldots \ldots \ldots \ldots \ldots \ldots$

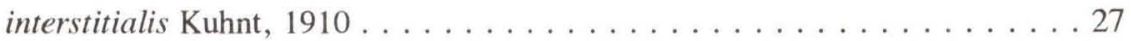

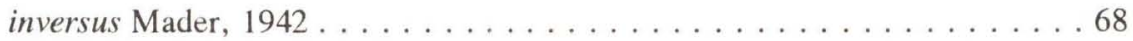

involutus Kuhnt, $1908 \ldots \ldots \ldots \ldots \ldots \ldots$. . . . . . . . . . . 92

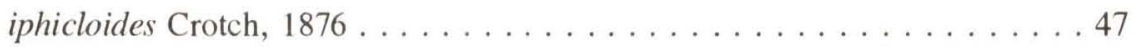

Iphiclus (s.str.) Chevr., $1837 \ldots \ldots \ldots \ldots \ldots$

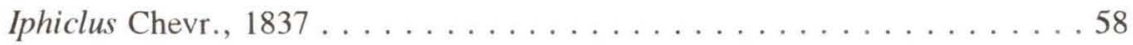

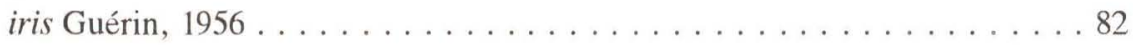

irroratus Kuhnt, $1908 \ldots \ldots \ldots \ldots$. . . . . . . . . . . . . . 104

irroratus Lac., $1842 \ldots \ldots \ldots \ldots$. . . . . . . . . . . . . . . . . 116

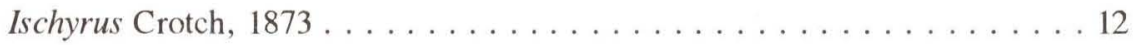

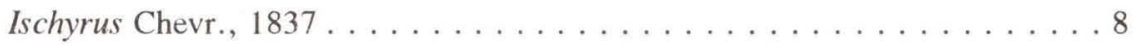

isequeboensis Voet, $1778 \ldots \ldots \ldots \ldots$. . . . . . . . . . . . 92

jacinthoi Alv., $1977 \ldots \ldots \ldots \ldots$. . . . . . . . . . . . . . . . . 82

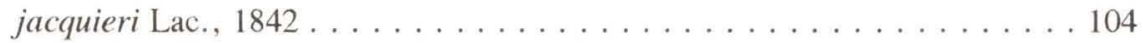

jansoni Crotch, 1873, (Aeg.) . . . . . . . . . . . . . . . . 54

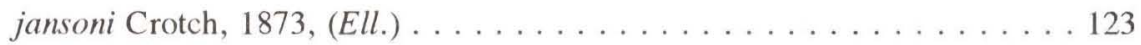

jansoni Crotch, 1873, (Olig.) . . . . . . . . . . . . . . . . 109

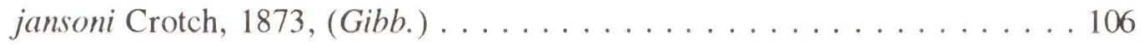

jaspidea Erichson, $1847 \ldots \ldots \ldots$. . . . . . . . . . . . . . 99

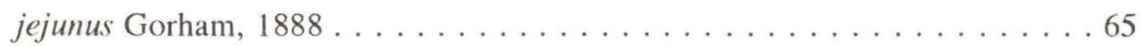

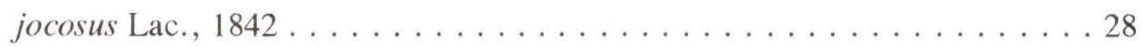

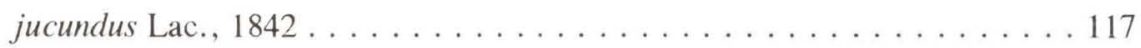

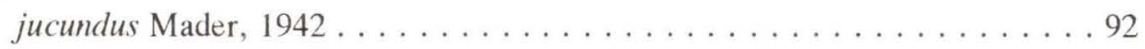

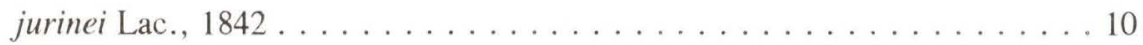

Kakoia Alv., $1971 \ldots \ldots \ldots$. . . . . . . . . . . . . . . 87

kempferi Alv., $1977 \ldots \ldots \ldots \ldots \ldots \ldots$

kirschi Crotch, $1876 \ldots \ldots \ldots \ldots \ldots \ldots$

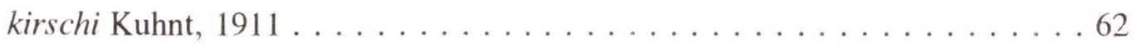

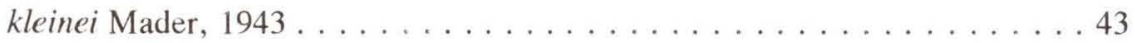

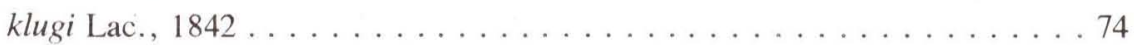


knochi, Lac., $1842 \ldots \ldots \ldots \ldots$. . . . . . . . . . . . . . . 10

kourouensis Lac., 1842 . . . . . . . . . . . . . . . . . . 65

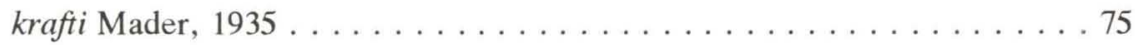

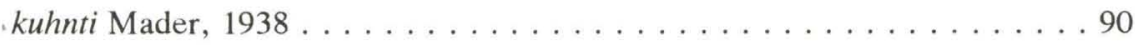

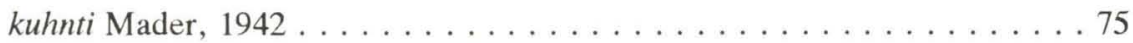

kuhnti Deelder, $1942 \ldots \ldots \ldots \ldots$. . . . . . . . . . . . . 92

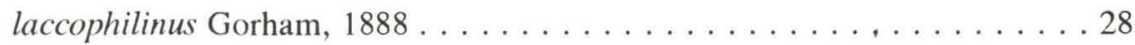

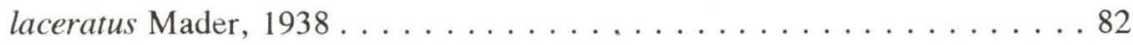

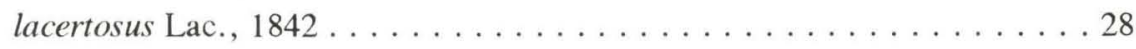

lacordairei Crotch, $1876 \ldots \ldots \ldots \ldots \ldots \ldots \ldots \ldots$

lacordairei Lac., 1842 . . . . . . . . . . . . . . . . . . . . . . 100

laetus Deelder, $1942 \ldots \ldots \ldots \ldots \ldots$. . . . . . . . . . . . . 92

laetus Crotch, $1876 \ldots \ldots \ldots \ldots \ldots$

laetus Schenkl., $1919 \ldots \ldots \ldots \ldots \ldots$

laetus Lac., $1842 \ldots \ldots \ldots \ldots$. . . . . . . . . . . . . . . . . . . 14

laevigatus Deelder, $1942 \ldots \ldots \ldots$. . . . . . . . . . . . . 107

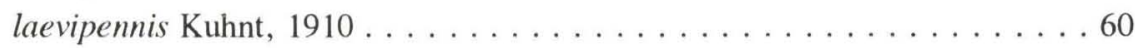

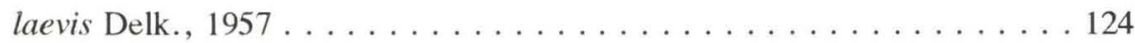

laeviusculus Crotch, $1876 \ldots \ldots \ldots \ldots \ldots \ldots \ldots$

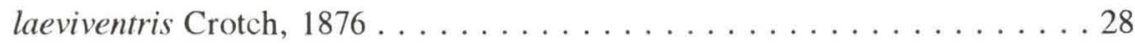

lateripunctatus Crotch, $1876 \ldots \ldots \ldots \ldots \ldots \ldots \ldots$

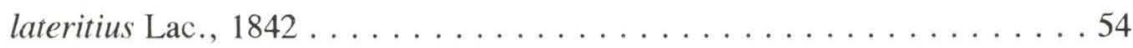

latifasciata Delk., $1952 \ldots \ldots \ldots \ldots \ldots \ldots \ldots \ldots \ldots$

latipalpus Johnson, $1967 \ldots \ldots \ldots \ldots$. . . . . . . . . . . . 40

latreillei Lac., $1842 \ldots \ldots \ldots \ldots \ldots$. . . . . . . . . . . . . . 92

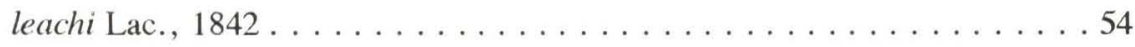

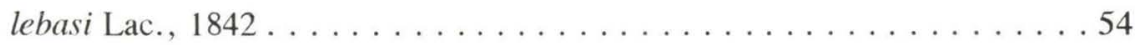

leoparda Lac., $1842 \ldots \ldots \ldots \ldots \ldots$. . . . . . . . . . 100

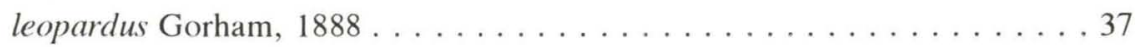

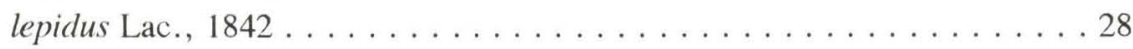

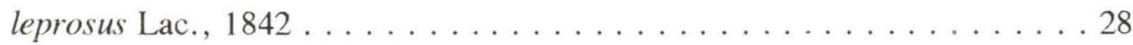

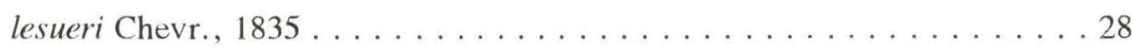

limbatus Kuhnt, 1910 . . . . . . . . . . . . . . . . . . . . . . . . 109

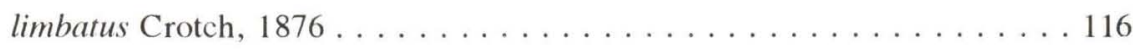

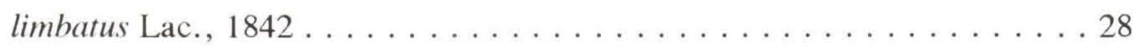

limbatus Oliv., $1792 \ldots \ldots \ldots \ldots \ldots \ldots \ldots \ldots$ 
lineaticollis Dup., $1825 \ldots \ldots \ldots \ldots \ldots$. . . . . . . . . . . . 121

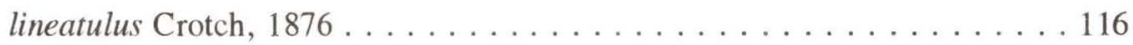

lineatus Guér.-Mén., $1844 \ldots \ldots \ldots \ldots$. . . . . . . . . . . . 54

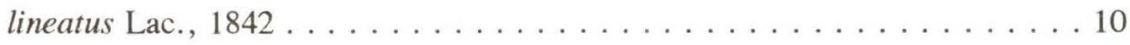

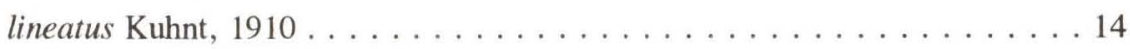

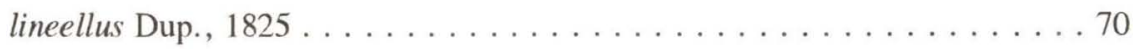

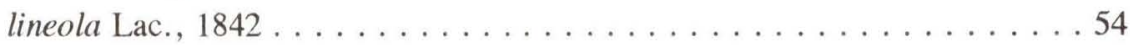

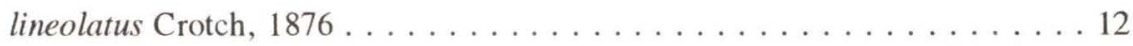

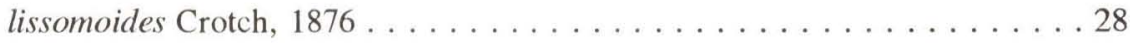

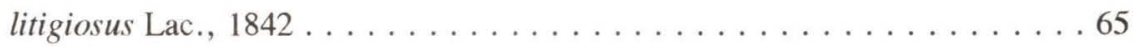

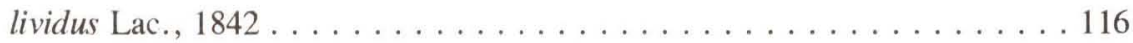

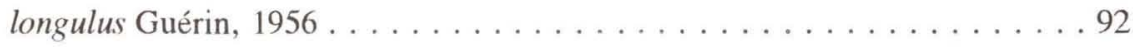

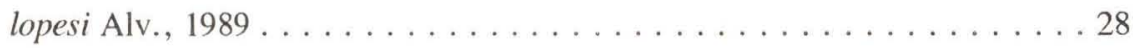

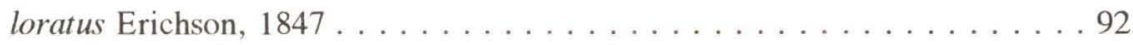

lucidus Lac., $1842 \ldots \ldots \ldots \ldots \ldots \ldots \ldots \ldots \ldots \ldots$

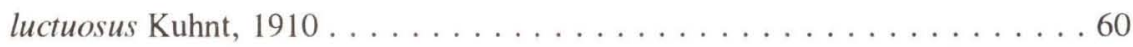

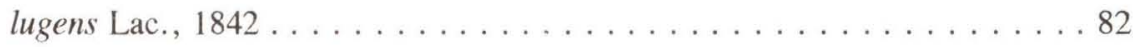

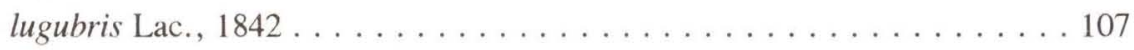

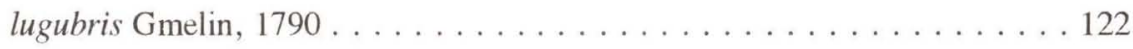

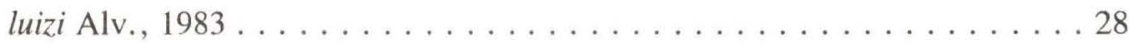

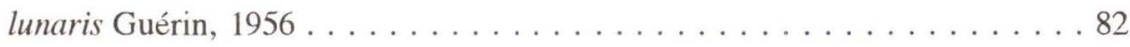

lunulatus Oliv., $1792 \ldots \ldots \ldots \ldots 7 . \ldots \ldots \ldots$

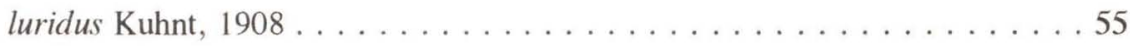

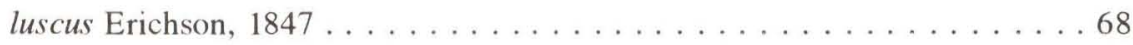

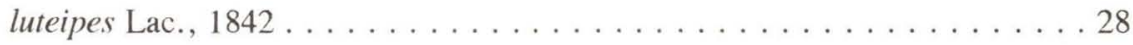

luteobifasciatus Mader, $1942 \ldots \ldots \ldots \ldots \ldots \ldots$

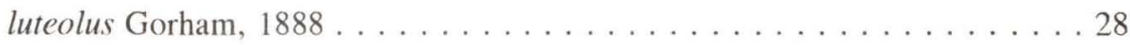

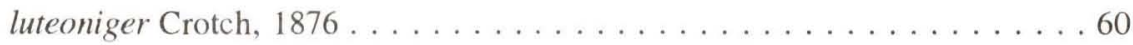

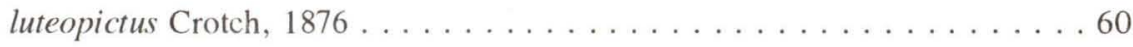

luteotaeniatus Kuhnt, $1908 \ldots \ldots \ldots \ldots$. . . . . . . . . . . 92

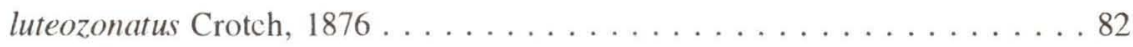

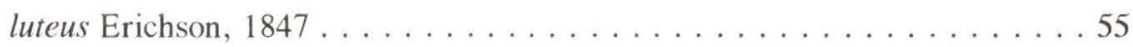

Lybanoides Gorham, $1888 \ldots \ldots \ldots \ldots \ldots \ldots \ldots \ldots$

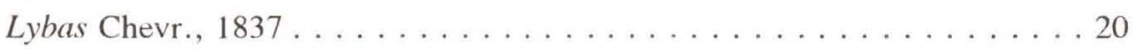

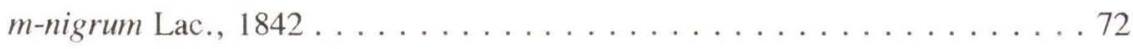

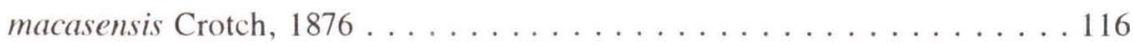




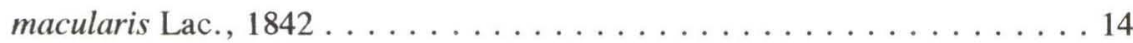
maculatoides Mader, $1943 \ldots \ldots \ldots \ldots \ldots \ldots \ldots \ldots$ maculatus Guér.-Mén., $1844 \ldots \ldots \ldots \ldots$. . . . . . . . . . 45

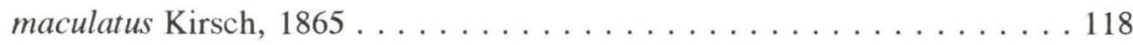

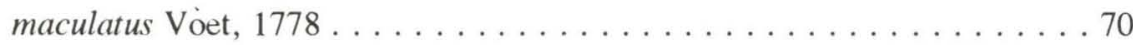

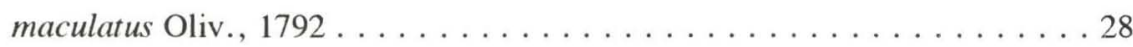
maculatus Guér.-Mén., $1841 \ldots \ldots \ldots \ldots \ldots \ldots$

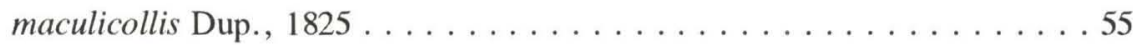
maculicollis Kuhnt, $1910 \ldots \ldots \ldots \ldots \ldots \ldots \ldots \ldots$ maculiforus Crotch, $1876 \ldots \ldots \ldots \ldots \ldots$

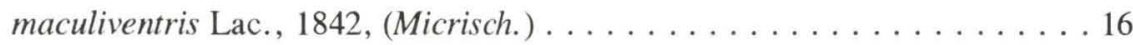

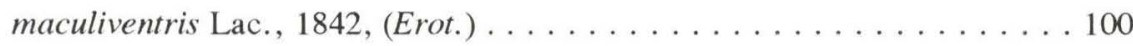

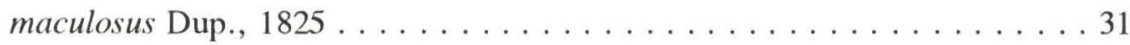
maderi Delk., 1957 . . . . . . . . . . . . . . . . . . . . . . . . . 39 maeander Lac., $1842 \ldots \ldots \ldots \ldots \ldots \ldots$. . . . . . . . . . . . 47 magus Lac., 1842 . . . . . . . . . . . . . . . . . . . . . . . . 29 major Mader, 1955 . . . . . . . . . . . . . . . . . . . . . 29 manicatus Lac., $1842 \ldots \ldots \ldots \ldots$. . . . . . . . . . . . . . . 65

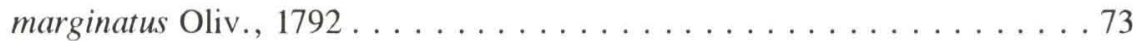
marginatus Guér.-Mén., 1841 . . . . . . . . . . . . . . . . . . . 119 margineguttatus Crotch, $1876 \ldots \ldots \ldots \ldots \ldots$ marginemaculatus Crotch, $1876 \ldots \ldots \ldots \ldots \ldots \ldots \ldots \ldots \ldots \ldots$

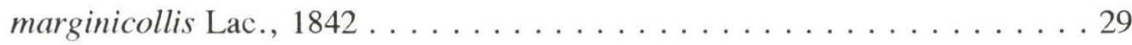
marmoratus Delk., 1957 . . . . . . . . . . . . . . . . . . . . . . . 124 marshami Lac., $1842 \ldots \ldots \ldots \ldots \ldots$. . . . . . . . . . . . . . 100

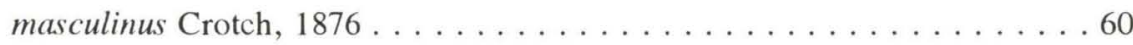

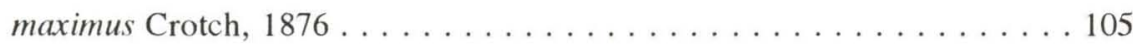

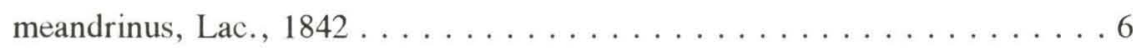

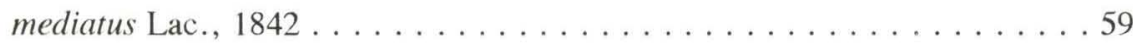
mediofasciatus Guérin, $1956 \ldots \ldots \ldots \ldots \ldots$ mediolineatus Mader, $1942 \ldots \ldots \ldots \ldots$. . . . . . . . . . . . 60

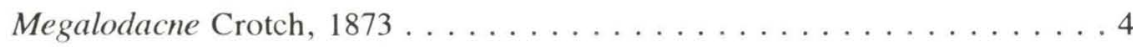
Megaprotus Lac., $1842 \ldots \ldots \ldots \ldots \ldots \ldots \ldots$ Megischyrus Crotch, $1873 \ldots \ldots \ldots \ldots \ldots \ldots \ldots$ melanocerus Lac., $1842 \ldots \ldots \ldots \ldots \ldots \ldots$. . . . . . . . . 41 melanocorynus Lac., $1842 \ldots \ldots \ldots \ldots$. . . . . . . . . . . . 44 
melanoderes Kuhnt, 1910, (Olig.) . . . . . . . . . . . . . . . . 109

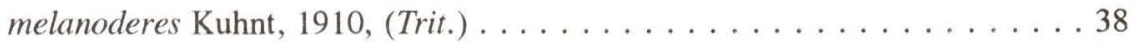

melanogaster Guér.-Mén., 1844 . . . . . . . . . . . . . . . . . . 19

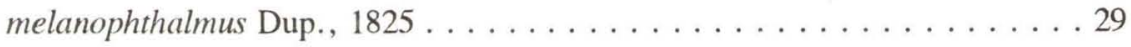

melanopterus Lac., $1842 \ldots \ldots \ldots \ldots \ldots$

melanopus Guér.-Mén., $1841 \ldots \ldots \ldots \ldots$

melanopus Lac., $1842 \ldots \ldots \ldots$. . . . . . . . . . . . . . . . . 49

melanopus Gorham, $1888 \ldots \ldots \ldots \ldots \ldots \ldots 77$

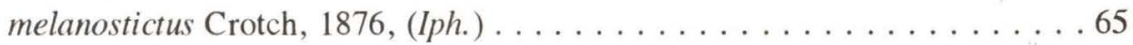

melanostictus Lac., $1842 \ldots \ldots \ldots \ldots \ldots$. . . . . . . . . . 31

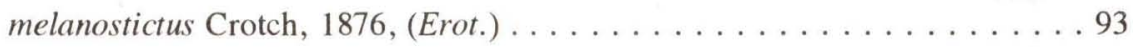

melanostigma Lac., $1842 \ldots \ldots \ldots \ldots \ldots \ldots \ldots$

melanotus Gorham, 1888 . . . . . . . . . . . . . . . . . . . . . 29

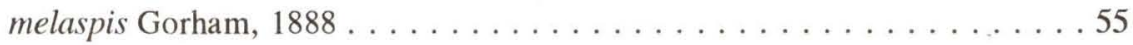

meleagris Lac., $1842 \ldots \ldots \ldots \ldots \ldots \ldots \ldots \ldots \ldots$

mendax Lac., $1842 \ldots \ldots \ldots \ldots$. . . . . . . . . . . . . . 65

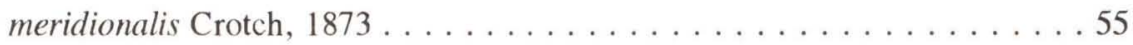

mesomelas Gorham, $1888 \ldots \ldots \ldots \ldots \ldots \ldots \ldots \ldots \ldots \ldots \ldots \ldots$

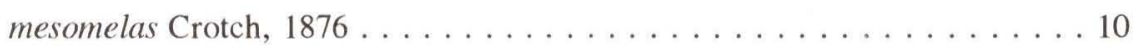

mesosternalis Schaef., $1905 \ldots \ldots \ldots$. . . . . . . . . . . 40

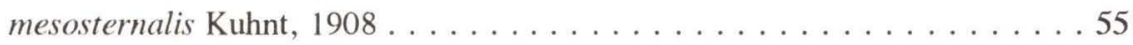

mexicanus Lac., $1842 \ldots \ldots \ldots \ldots \ldots$

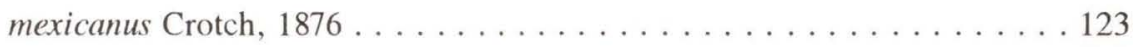

Micrerotylus Crotch, $1876 \ldots \ldots \ldots \ldots \ldots \ldots$

Micrischyrus Alv., $1965 \ldots \ldots \ldots \ldots \ldots \ldots \ldots$

microguttata Kuhnt, $1908 \ldots \ldots \ldots \ldots \ldots \ldots \ldots$

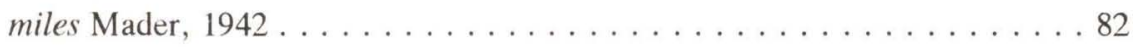

miliaris Lac., $1842 \ldots \ldots \ldots \ldots$. . . . . . . . . . . . . . . 105

militarioides Mader, 1942 . . . . . . . . . . . . . . . . . . . . . . 109

militaris Germ., 1842 . . . . . . . . . . . . . . . . . . . . . . . . 109

miniatus Lac., 1842 . . . . . . . . . . . . . . . . . . . . . . . . . . 29

minor Deelder, $1942 \ldots \ldots \ldots \ldots$. . . . . . . . . . . . . . . 91

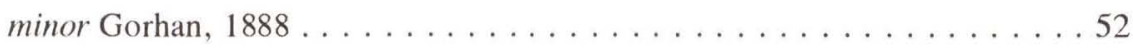

minor Kuhnt, $1908 \ldots \ldots \ldots \ldots \ldots \ldots \ldots$

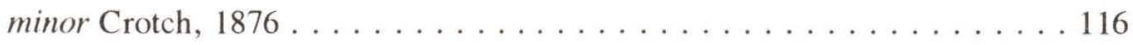

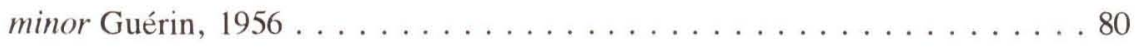


minutus Dup., $1825 \ldots \ldots \ldots$. . . . . . . . . . . . . . . . . . 29

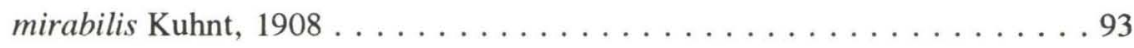

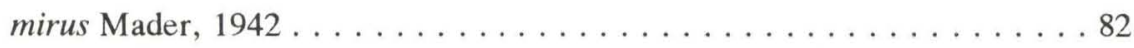

misellus Lac., 1842 . . . . . . . . . . . . . . . . . . . . . . . . 29

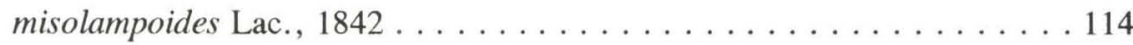

modestus Oliv., $1807 \ldots \ldots \ldots$. . . . . . . . . . . . . . . . 18

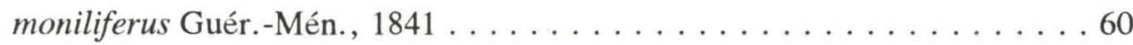

monochrous Lac., $1842 \ldots \ldots \ldots \ldots$. . . . . . . . . . . . . . 55

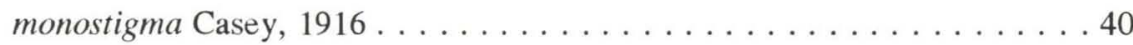

monrosi Guérin, 1949 . . . . . . . . . . . . . . . . . . . . . . . 29

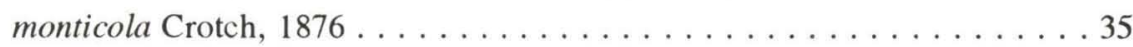

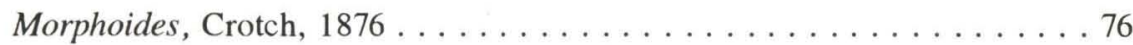

Morphoides, Gemm. \& Har., $1876 \ldots \ldots \ldots$. . . . . . . . . . . 58

Morphoides Hope, $1841 \ldots \ldots$. . . . . . . . . . . . . . . . . . 73

multifida Lac., $1842 \ldots \ldots \ldots \ldots \ldots \ldots \ldots \ldots \ldots$

multiguttata Lac., $1842 \ldots \ldots \ldots \ldots$. . . . . . . . . . . . . 100

multiguttatus Gorham, $1888 \ldots \ldots \ldots \ldots \ldots \ldots \ldots \ldots \ldots \ldots \ldots \ldots$

multimaculatus Tasch., $1870 \ldots \ldots$. . . . . . . . . . . . . . . . 29

multinotatus Lac., $1842 \ldots \ldots \ldots \ldots$. . . . . . . . . . . . . . . 118

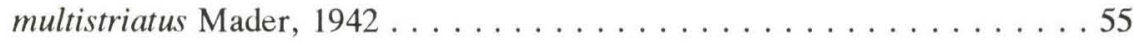

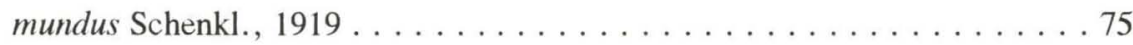

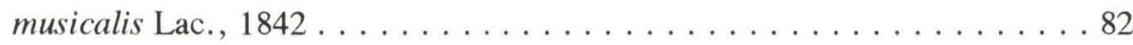

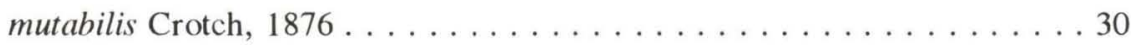

mutabilis Gorham, $1889 \ldots \ldots \ldots \ldots \ldots \ldots \ldots$

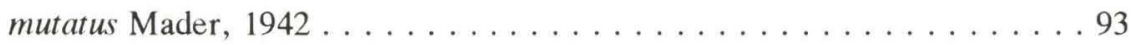

mycetophagoides Crotch, $1876 \ldots \ldots \ldots \ldots$

mycetophiloides Crotch, $1876 \ldots \ldots \ldots \ldots \ldots \ldots$

mycetophilus Lac., $1842 \ldots \ldots \ldots \ldots \ldots$

Mycolybas Crotch, $1876 \ldots \ldots \ldots \ldots \ldots \ldots \ldots$

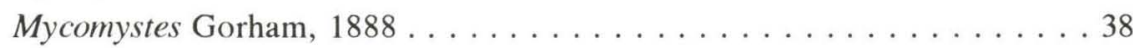

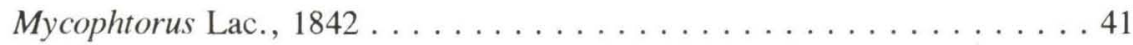

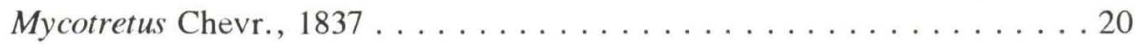

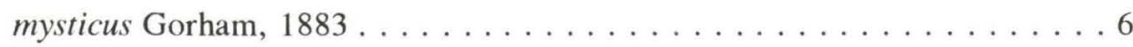

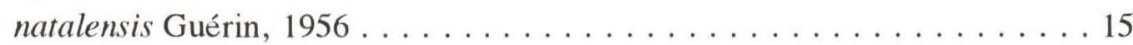

nauteae Crotch, $1876 \ldots \ldots \ldots \ldots$. . . . . . . . . . . . . . . . . . . . . .

navicularis Lac., $1842 \ldots \ldots \ldots \ldots \ldots \ldots$ 
Neacronotus Alv., 1965 . . . . . . . . . . . . . . . . . . . . 67

nebrioides Crotch, $1876 \ldots \ldots \ldots \ldots$. . . . . . . . . . . 45

nebulosus Guér. - Mén., 1841 . . . . . . . . . . . . . . . . . . 75

neglectus Guérin, 1956 . . . . . . . . . . . . . . . . . . . 77

neglectus Mader, $1942 \ldots \ldots \ldots \ldots \ldots \ldots$

neglectus Kuhnt, $1908 \ldots \ldots \ldots \ldots$. . . . . . . . . . . . . 103

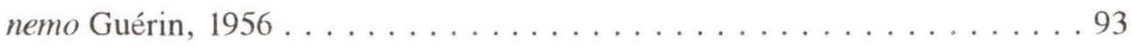

Neobarytopus Alv., 1965 . . . . . . . . . . . . . . . . . . 79

Neogaster Alv., $1965 \ldots \ldots \ldots$. . . . . . . . . . . . . . . . 73

Neomorphoides Alv., $1977 \ldots \ldots \ldots \ldots$

neophyta Lac., $1842 \ldots \ldots \ldots \ldots$. . . . . . . . . . . . . . 83

Neopriotelus Alv., 1965 . . . . . . . . . . . . . . . . . . . 115

Neoxestus Crotch, $1876 \ldots \ldots \ldots$. . . . . . . . . . . . . . . 45

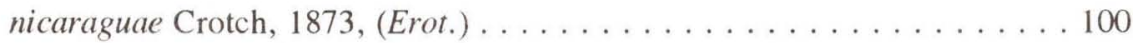

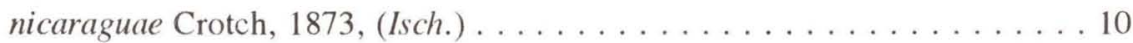

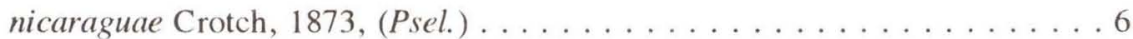

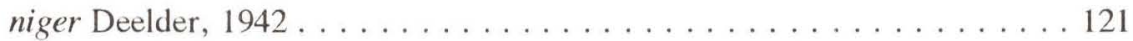

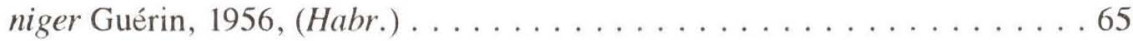

niger Guérin, 1956, (Neom.) . . . . . . . . . . . . . . . . . . 77

niger Kuhnt, $1908 \ldots \ldots \ldots \ldots$. . . . . . . . . . . . . . . . . . 97

nigricollis Kuhnt, $1908 \ldots \ldots \ldots \ldots \ldots \ldots 2$

nigricollis Gorham, $1888 \ldots \ldots \ldots \ldots \ldots \ldots \ldots \ldots \ldots \ldots \ldots \ldots \ldots \ldots$

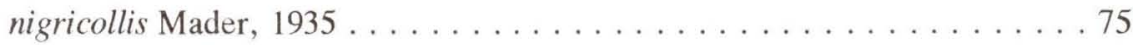

nigripennis Demay, $1838 \ldots \ldots \ldots \ldots \ldots \ldots \ldots \ldots \ldots \ldots \ldots \ldots$

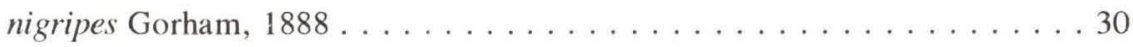

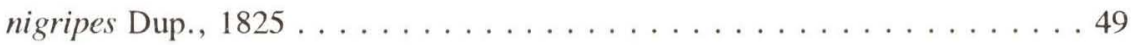

nigripes Mader, $1942 \ldots \ldots \ldots \ldots \ldots \ldots \ldots \ldots$

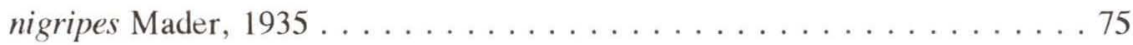

nigritarsis Mader, $1942 \ldots \ldots \ldots \ldots \ldots \ldots \ldots \ldots$

nigriventris Crotch, $1876 \ldots \ldots \ldots \ldots \ldots \ldots \ldots$

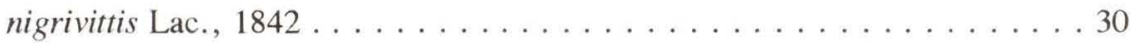

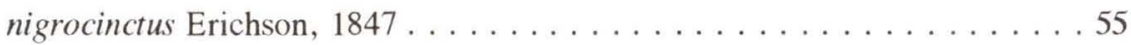

nigrocinctus Lac., $1842 \ldots \ldots \ldots \ldots \ldots \ldots \ldots$

nigrocinctus Kuhnt, $1908 \ldots \ldots \ldots$. . . . . . . . . . . . . . . . 93

nigrofasciatus Deelder, 1942 . . . . . . . . . . . . . . . . . . . 92

nigrolimbatus Mader, $1942 \ldots \ldots \ldots \ldots \ldots$ 


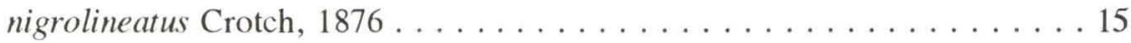

nigromaculatus Kuhnt, $1909 \ldots \ldots \ldots \ldots \ldots$

nigromanicatus Boyle, $1954 \ldots \ldots \ldots \ldots$

nigromarmoratus Mader, $1942 \ldots \ldots \ldots \ldots \ldots \ldots$

nigronotatus Gorham, $1888 \ldots \ldots \ldots \ldots \ldots \ldots \ldots \ldots \ldots \ldots$

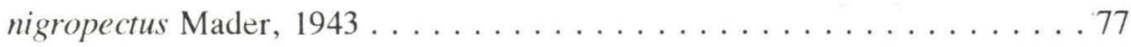

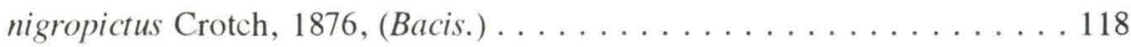

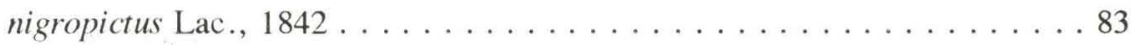

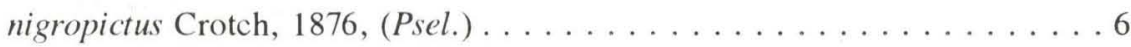

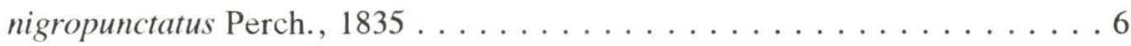

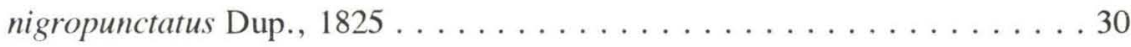

nigrotaeniatus Lac., 1842 . . . . . . . . . . . . . . . . . . . . . 109

nigroterminalis Kuhnt, $1908 \ldots \ldots \ldots \ldots \ldots \ldots$

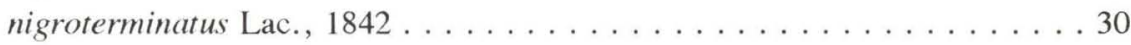

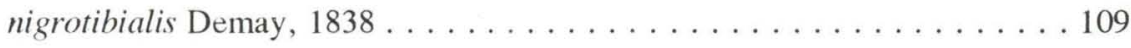

nigrotibialis Mader, $1942 \ldots \ldots \ldots$. . . . . . . . . . . . . . . 49

nigrotibialis Crotch, $1876 \ldots \ldots \ldots \ldots \ldots \ldots \ldots \ldots$

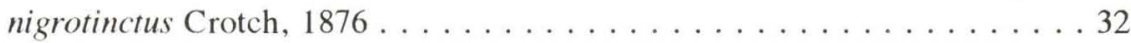

nigrotrifasciatus Mader, $1942 \ldots \ldots \ldots \ldots \ldots \ldots \ldots \ldots \ldots \ldots$

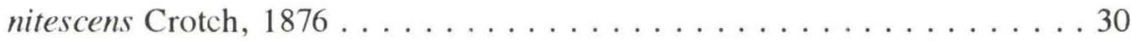

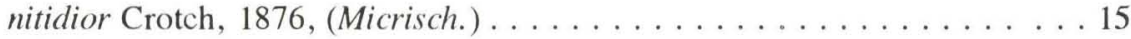

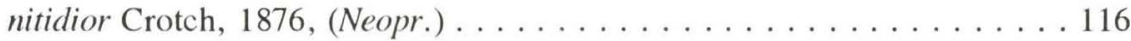

nitidulus Oliv., $1807 \ldots \ldots \ldots$. . . . . . . . . . . . . . . . 83

nitidus Mader, $1942 \ldots \ldots \ldots \ldots \ldots \ldots \ldots$

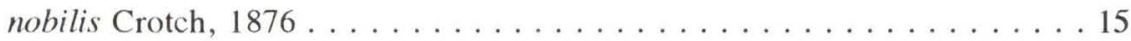

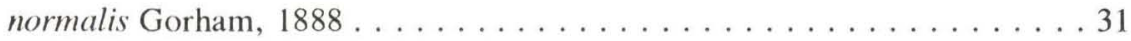

normalis Lac . $1842 \ldots \ldots \ldots \ldots \ldots$. . . . . . . . . . . . . 43

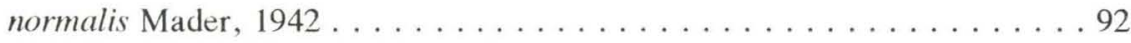

notatus Oliv., $1792 \ldots \ldots \ldots \ldots \ldots \ldots \ldots \ldots \ldots \ldots \ldots \ldots$

noterinus Gorham, $1888 \ldots \ldots \ldots \ldots \ldots \ldots \ldots \ldots \ldots \ldots$

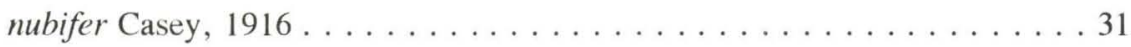

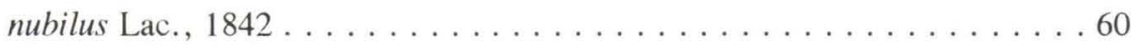

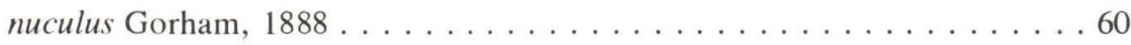

nugator Lac., $1842 \ldots \ldots \ldots \ldots \ldots \ldots \ldots \ldots \ldots \ldots \ldots \ldots \ldots \ldots \ldots$

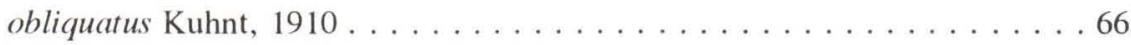

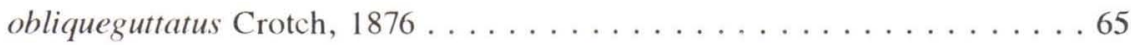

Revta bras. Zool. 11 (1): 1 - 175, 1994 


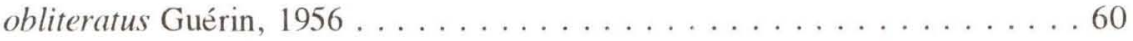

obliteratus Erichson, $1847 \ldots \ldots \ldots \ldots \ldots \ldots$

oblitus Guérin, $1956 \ldots \ldots \ldots \ldots \ldots \ldots$

oblitus Lac., $1842 \ldots \ldots \ldots \ldots \ldots$. . . . . . . . . . . . . . . . . 65

oblongonotatus Lac., $1842 \ldots \ldots \ldots \ldots$

oblongosignatus Guér-- Mén., $1844 \ldots \ldots \ldots \ldots \ldots \ldots$

oblongus Dup., $1825 \ldots \ldots \ldots \ldots \ldots \ldots \ldots \ldots \ldots \ldots \ldots \ldots \ldots$

obscurior Curran, $1941 \ldots \ldots \ldots \ldots \ldots \ldots \ldots \ldots \ldots$

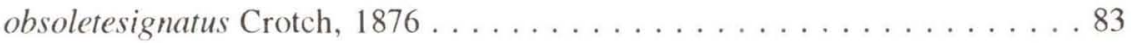

obsoletus Crotch, $1876 \ldots \ldots \ldots \ldots \ldots$

obstinatus Mader, $1942 \ldots \ldots \ldots \ldots \ldots$

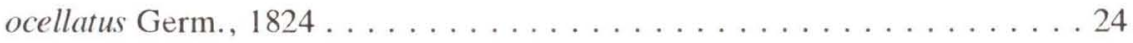

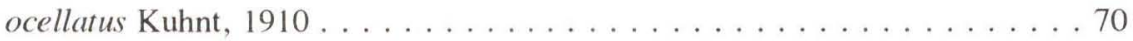

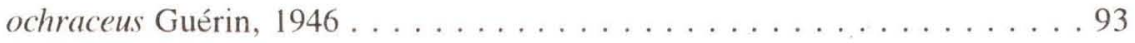

octodecimguttatus Lac., $1842 \ldots \ldots \ldots \ldots \ldots \ldots \ldots$

octoguttatus Oliv., $1807 \ldots \ldots \ldots \ldots$. . . . . . . . . . . 83

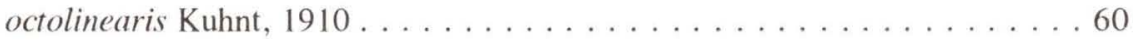

octomaculatus Oliv., $1792 \ldots \ldots \ldots \ldots$. . . . . . . . . . 116

octomaculatus Kuhnt, 1910 . . . . . . . . . . . . . . . . . . 118

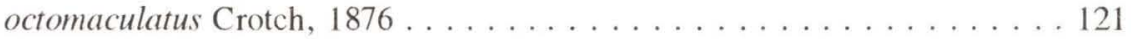

octopunctatus Kirsch, $1876 \ldots \ldots \ldots$. . . . . . . . . . . . . 60

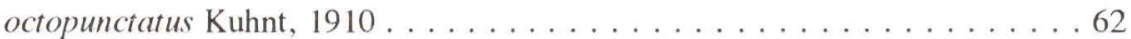

octopunctatus Erichson, $1847 \ldots \ldots \ldots \ldots \ldots$. . . . . . . . 47

octopustulatus Guérin, $1956 \ldots \ldots \ldots \ldots \ldots \ldots \ldots \ldots \ldots \ldots \ldots$

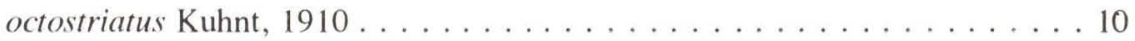

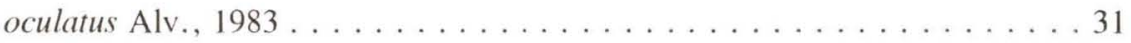

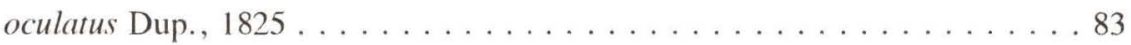

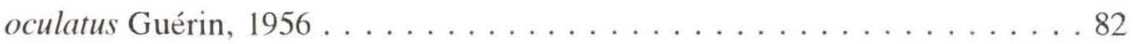

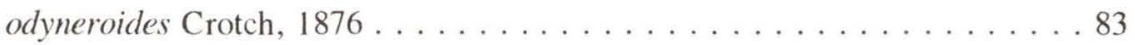

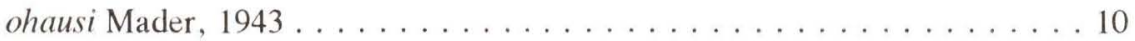

Oligocorynus Chevr., 1837 . . . . . . . . . . . . . . . . . . . 107

olivaceipennis Mader, $1942 \ldots \ldots \ldots \ldots \ldots \ldots$

olivieri Lac., $1842 \ldots \ldots \ldots \ldots$. . . . . . . . . . . . . . . . . . 94

Omoiotelus Hope, 1841 . . . . . . . . . . . . . . . . . . . 120

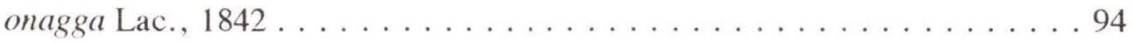

Oocyanus Hope, 1841 . . . . . . . . . . . . . . . . . . . . . 17 
Oogaster Lac., $1842 \ldots \ldots \ldots \ldots \ldots \ldots$. . . . . . . . . . . . . 73

opacus Schaef., $1915 \ldots \ldots \ldots$. . . . . . . . . . . . . . . . 39

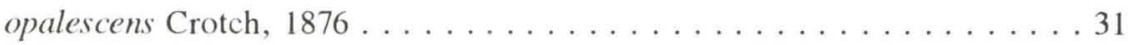

opalizans Lac., $1842 \ldots \ldots \ldots \ldots \ldots \ldots \ldots \ldots \ldots \ldots$

opalizans Mader, $1942 \ldots \ldots \ldots \ldots \ldots \ldots \ldots \ldots \ldots \ldots \ldots \ldots \ldots$

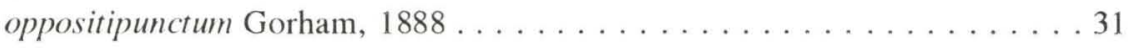

orbignyanus Lac., $1842 \ldots \ldots \ldots \ldots \ldots \ldots \ldots \ldots \ldots \ldots \ldots \ldots \ldots$

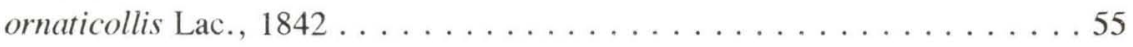

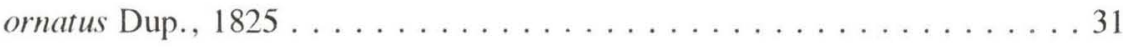

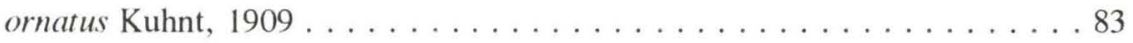

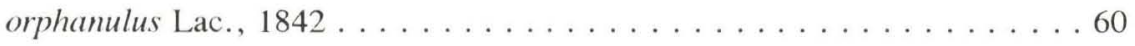

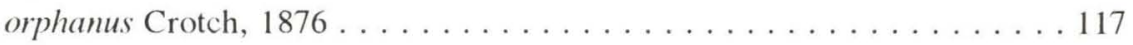

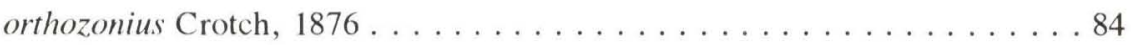

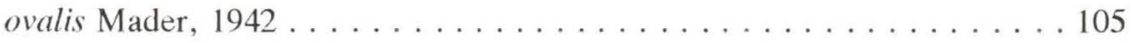

ovatus Crotch, $1876 \ldots \ldots \ldots \ldots \ldots \ldots \ldots \ldots \ldots$

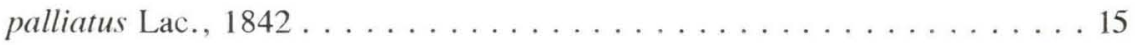

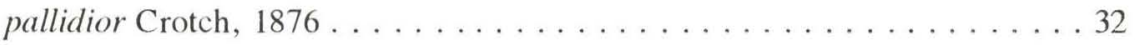

pallidipennis Gorham, $1888 \ldots \ldots \ldots \ldots \ldots \ldots \ldots \ldots \ldots \ldots \ldots \ldots \ldots \ldots$

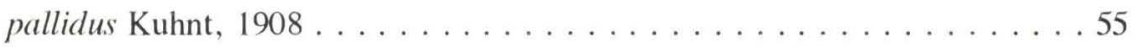

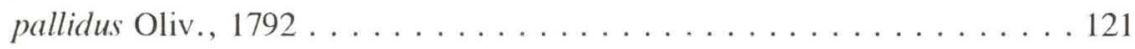

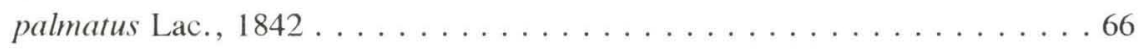

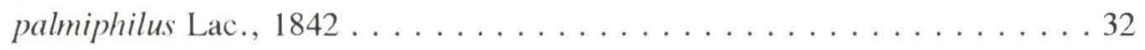

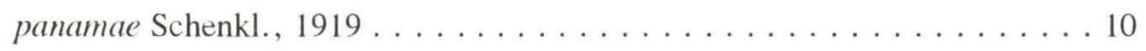

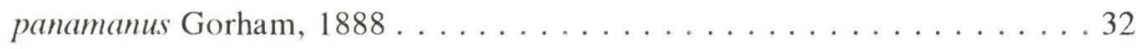

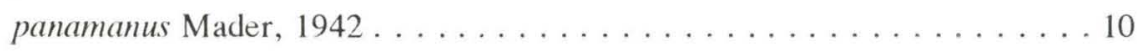

pantherinus Kuhnt, $1909 \ldots \ldots \ldots \ldots \ldots$. . . . . . . . . . . . . . . .

papulosus Lac., $1842 \ldots \ldots \ldots \ldots \ldots \ldots$. . . . . . . . . . . . 94

paraensis Alv., $1976 \ldots \ldots \ldots \ldots \ldots \ldots . \ldots \ldots . \ldots \ldots$

paraensis Guérin, $1956 \ldots \ldots \ldots \ldots \ldots \ldots \ldots$

paraguayanus Mader, $1943 \ldots \ldots \ldots \ldots \ldots \ldots \ldots$

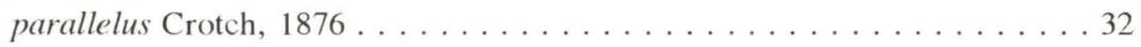

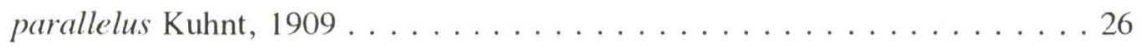

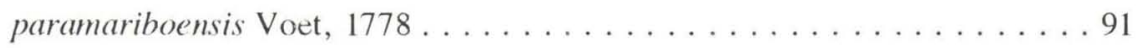

Paratritoma Gorham, $1888 \ldots \ldots \ldots \ldots \ldots$

parcepunctatus Crotch, $1876 \ldots \ldots \ldots \ldots \ldots \ldots$

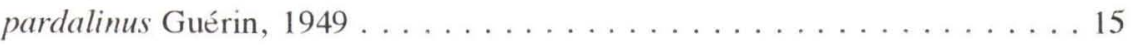

Revta bras. Zool. 11 (1): 1 - 175, 1994 
pardalinus Lac, $1842 \ldots \ldots \ldots \ldots \ldots \ldots \ldots \ldots \ldots \ldots$

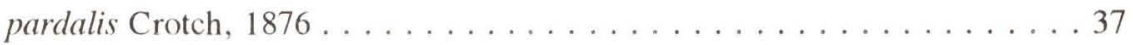

pardalis Erichson, $1848 \ldots \ldots \ldots \ldots \ldots \ldots \ldots \ldots$

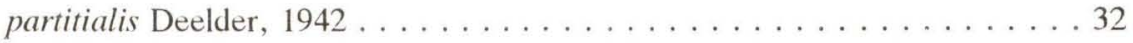

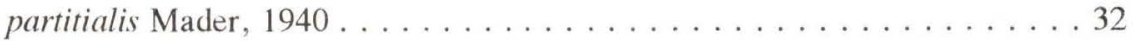

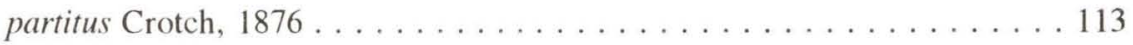

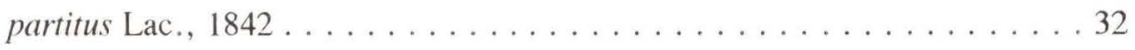

parvus Kuhnt, $1908 \ldots \ldots \ldots \ldots \ldots \ldots$. . . . . . . . . . . . 94

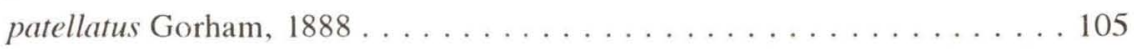

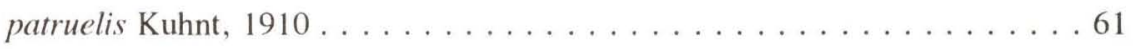

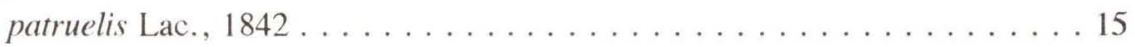

paucipunctatus Kuhnt, $1908 \ldots \ldots \ldots \ldots \ldots$. . . . . . . . . 97

pauper Guérin, $1956 \ldots \ldots \ldots \ldots \ldots \ldots \ldots \ldots \ldots \ldots$

pauperculus Lac., $1842 \ldots \ldots \ldots \ldots \ldots \ldots \ldots \ldots \ldots \ldots$

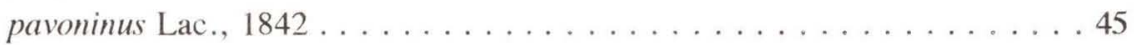

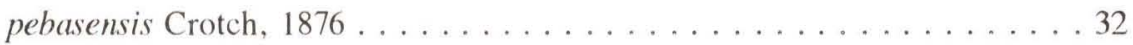

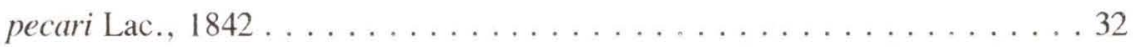

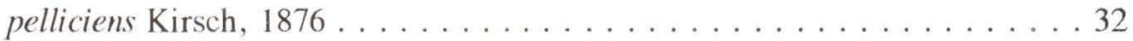

pentastictus Harold, $1875 \ldots \ldots \ldots \ldots \ldots \ldots$

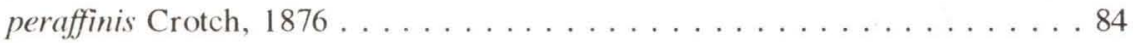

peregrinus Lac., $1842 \ldots \ldots \ldots \ldots \ldots \ldots$. . . . . . . . . . . 109

peregrinus Mader, $1942 \ldots \ldots \ldots \ldots \ldots . \ldots \ldots 4$

Perithonius Crotch, $1876 \ldots \ldots \ldots \ldots \ldots \ldots 14$

perizonatus Lac., $1842 \ldots \ldots \ldots \ldots \ldots \ldots \ldots \ldots \ldots \ldots \ldots \ldots \ldots$

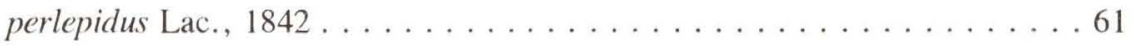

permutatus Kuhnt, $1908 \ldots \ldots \ldots \ldots \ldots \ldots$. . . . . . . . . 94

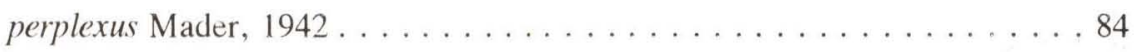

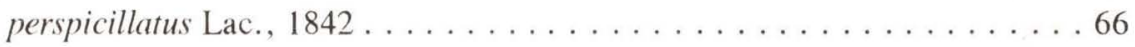

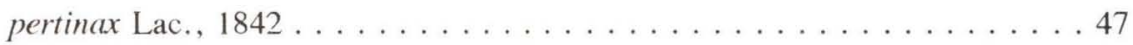

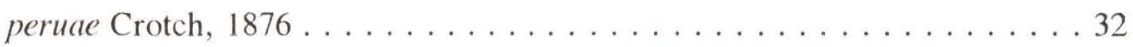

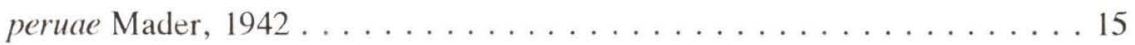

peruvianu Kirsch, 1876, (Mycoph.) . . . . . . . . . . . . . 41

peruvianus Crotch, 1876, (Erot.) .. . . . . . . . . . . . . . . 94

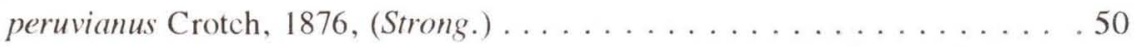

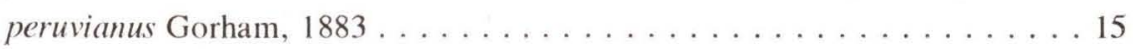

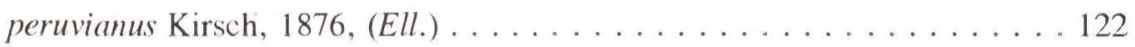




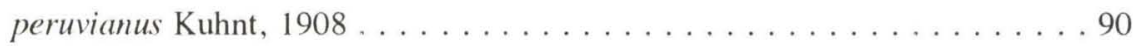

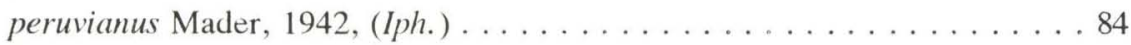

peruvianus Mader, 1942, (Neopr.) . . . . . . . . . . . . . 116

peruvianus Mader, 1942, (Olig.) . . . . . . . . . . . . . . . . . . 109

perversus Gorham, $1889 \ldots \ldots \ldots \ldots$. . . . . . . . . . . 68

Phricobacis Crotch, $1876 \ldots \ldots \ldots \ldots \ldots$

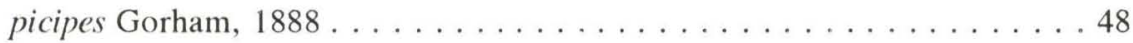

pictipennis Schenkl., $1919 \ldots \ldots \ldots \ldots \ldots \ldots \ldots \ldots \ldots$

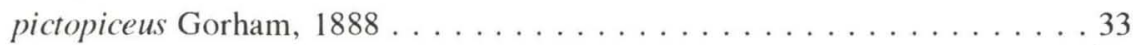

picturatus Crotch, $1876 \ldots \ldots \ldots$. . . . . . . . . . . . . . 94

picturatus Guérin, $1956 \ldots \ldots \ldots$. . . . . . . . . . . . . 79

pictus Gorham, 1887 . . . . . . . . . . . . . . . . . . . . 15

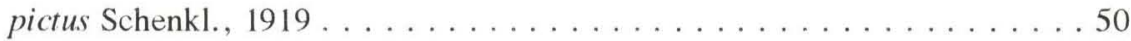

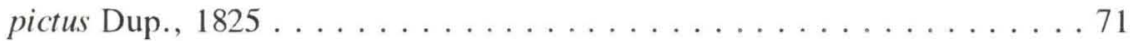

pithecius Lac., 1842 . . . . . . . . . . . . . . . . . . . . . . 61

placitus Kirsch, $1876 \ldots \ldots \ldots \ldots \ldots \ldots \ldots$

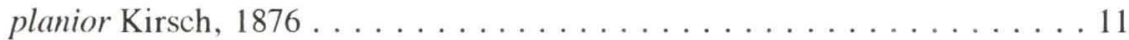

planipennis Kuhnt, 1909 . . . . . . . . . . . . . . . . . . . 84

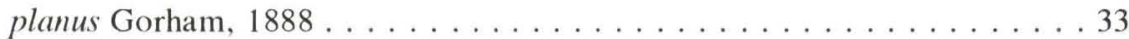

Plastococcus Gorham, 1898 . . . . . . . . . . . . . . . . . . 50

poecilosomus Lac., $1842 \ldots \ldots \ldots \ldots \ldots \ldots \ldots \ldots$

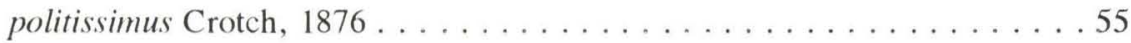

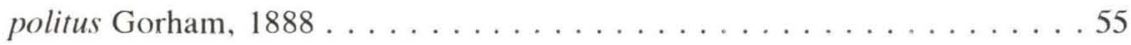

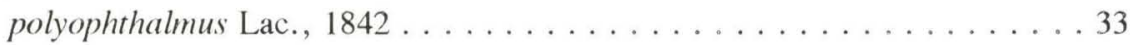

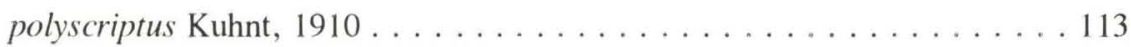

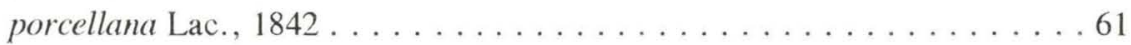

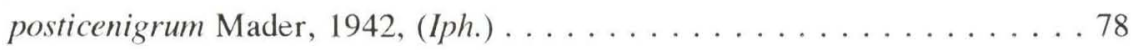

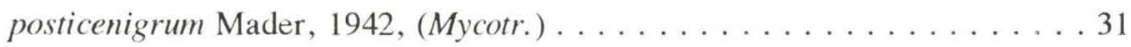

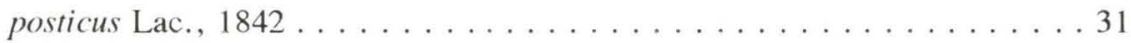

praenobilis Delk., $1957 \ldots \ldots \ldots \ldots$

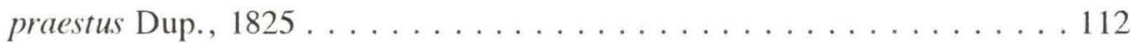

Prepopharus Erichson, $1847 \ldots \ldots \ldots \ldots \ldots \ldots \ldots \ldots$

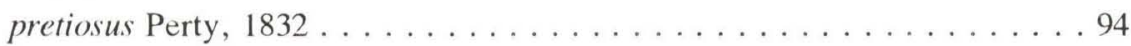

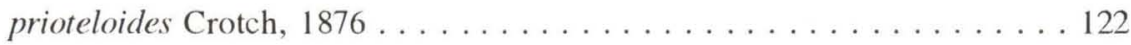

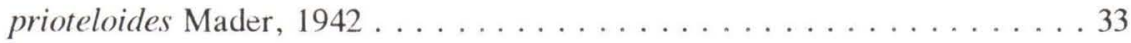

Priotelus Hope, 1841 . . . . . . . . . . . . . . . . . . . . . . 115 
procerus Erichson, $1847 \ldots \ldots \ldots \ldots \ldots \ldots \ldots \ldots$

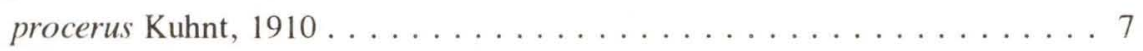

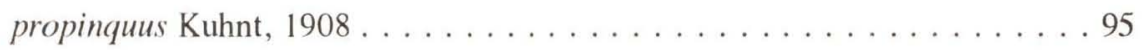

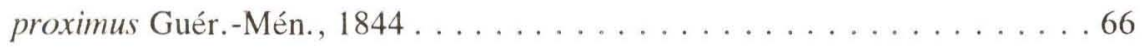

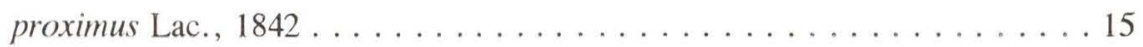

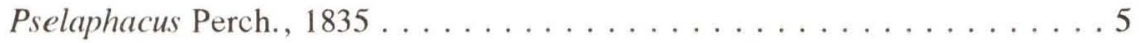

Psephodacne Heller, $1918 \ldots \ldots \ldots \ldots \ldots \ldots$

Pseudochrysomela Voet, $1778 \ldots \ldots \ldots 8$

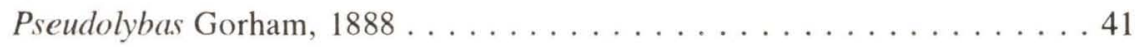

pseudomelanostictus Guérin, $1946 \ldots \ldots \ldots \ldots \ldots$. . . . . . . . 95

psittacus Lac., $1842 \ldots \ldots \ldots \ldots \ldots \ldots \ldots \ldots \ldots \ldots \ldots \ldots \ldots$

psylloboroides Crotch, $1876 \ldots \ldots \ldots \ldots \ldots \ldots \ldots \ldots \ldots \ldots$

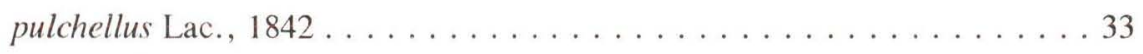

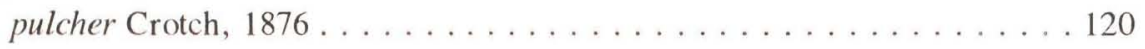

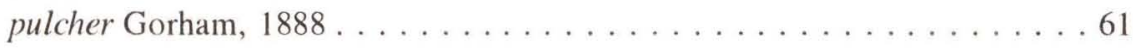

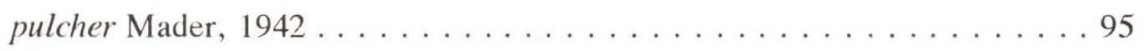

pulicarius Lac., 1842, (Apol.) . . . . . . . . . . . . . . . 43

pulicarius Lac., 1842, (Mycotr.) . . . . . . . . . . . . . . 26

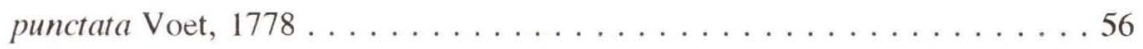

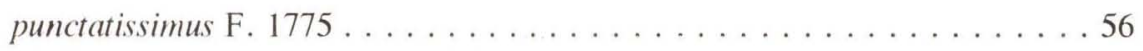

punctatostriatus Crotch, $1876 \ldots \ldots \ldots \ldots \ldots \ldots$

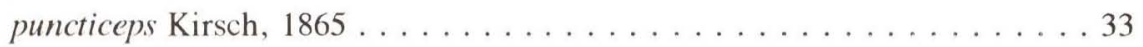

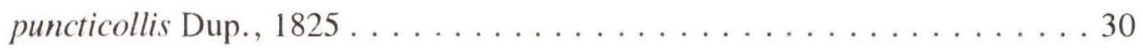

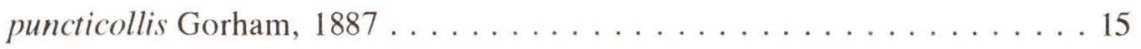

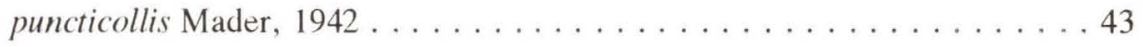

puncticollis Kirsch, $1876 \ldots \ldots \ldots \ldots \ldots \ldots \ldots \ldots$

puncticollis Guér.- Mén., $1841 \ldots \ldots \ldots \ldots \ldots \ldots \ldots$

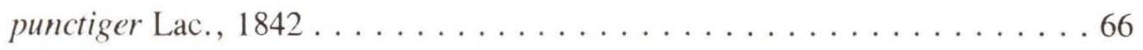

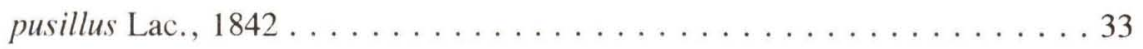

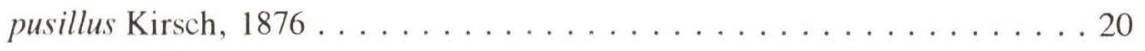

pustulatus Dup., $1825 \ldots \ldots \ldots$. . . . . . . . . . . . . . . . 99

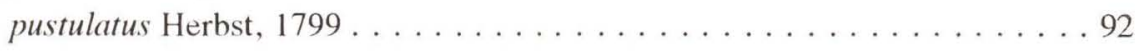

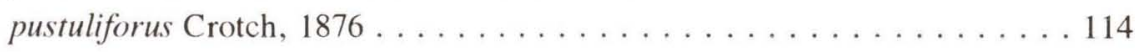

pygmaeus Lac., $1842 \ldots \ldots \ldots \ldots \ldots \ldots \ldots \ldots \ldots \ldots \ldots \ldots \ldots \ldots$

pyrrhocephalus Erichson, $1847 \ldots \ldots \ldots \ldots \ldots \ldots \ldots$

quadricruentatus Alv., $1976 \ldots \ldots \ldots \ldots \ldots$ 
quadrifasciata Crotch, $1876 \ldots \ldots \ldots \ldots \ldots \ldots$

quadrifasciatus Kirsch, $1865 \ldots \ldots \ldots \ldots \ldots$. . . . . . . . . . . . . .

quadriguttata Oliv., $1792 \ldots \ldots \ldots \ldots \ldots \ldots$

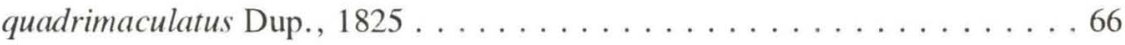

quadrimaculatus Gistel, $1848 \ldots \ldots \ldots \ldots \ldots \ldots \ldots \ldots$

quadrimaculatus Guérin, 1956, (Neopr.) . . . . . . . . . . . . . 117

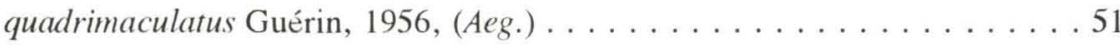

quadrimaculatus Guérin, $1948 \ldots \ldots \ldots \ldots \ldots \ldots$

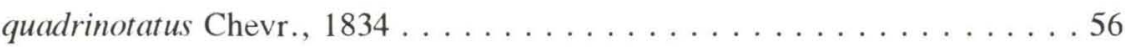

quadrinus Lac., $1842 \ldots \ldots \ldots$. . . . . . . . . . . . . . . . . 29

quadrioculatus Alv., $1983 \ldots \ldots \ldots \ldots \ldots \ldots \ldots \ldots \ldots$

quadriplagiatus Lac., $1842 \ldots \ldots \ldots \ldots \ldots \ldots \ldots \ldots \ldots$

quadripunctatus Crotch, $1876 \ldots \ldots \ldots \ldots \ldots \ldots \ldots \ldots \ldots$

quadripunctatus Oliv., $1792 \ldots \ldots \ldots \ldots \ldots \ldots$

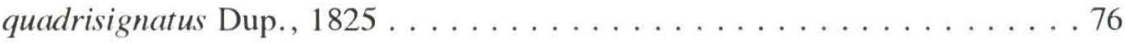

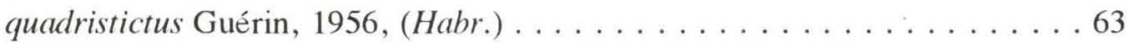

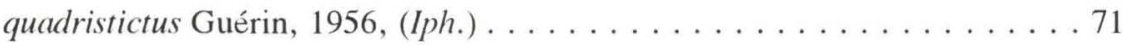

quadristriolatus Kuhnt, $1910 \ldots \ldots \ldots \ldots \ldots \ldots \ldots \ldots \ldots \ldots$

quattuordecimguttatus Lac., $1842 \ldots \ldots \ldots \ldots \ldots \ldots \ldots$

quinquefasciata Kuhnt, $1908 \ldots \ldots \ldots \ldots$. . . . . . . . . . . 94

quinquefasciatoides Mader, $1942 \ldots \ldots \ldots \ldots \ldots \ldots \ldots \ldots$

quinquefasciatus Lac., $1842 \ldots \ldots \ldots \ldots \ldots \ldots \ldots \ldots$

quinquenotatus Lac., $1842 \ldots \ldots \ldots \ldots \ldots \ldots \ldots \ldots$

quinquepunctatus Kuhnt, $1908 \ldots \ldots \ldots \ldots \ldots$

quinquepunctatus L., $1767 \ldots \ldots \ldots \ldots \ldots \ldots$

quinquepunctatus Gorham, $1887 \ldots \ldots \ldots \ldots \ldots \ldots$

quinquevingitipunctatus Mader, $1943 \ldots \ldots \ldots \ldots \ldots \ldots \ldots \ldots$

ramosus Oliv., $1807 \ldots$. . . . . . . . . . . . . . . . . . . . . . 84

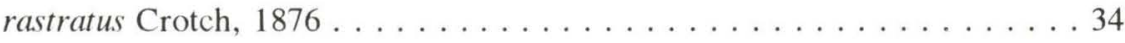

ratzeburgi Lac., 1842 . . . . . . . . . . . . . . . . . . . . . . 120

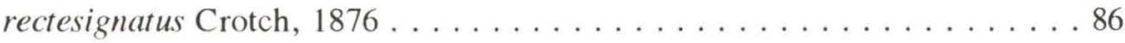

rediviva Gorham, $1888 \ldots \ldots \ldots \ldots \ldots \ldots \ldots \ldots$

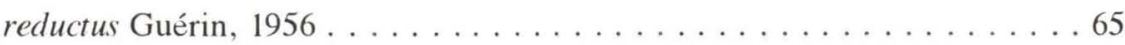

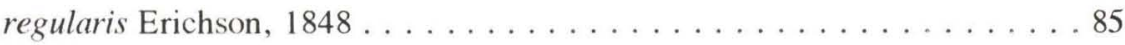

reichei Guér.-Mén., $1841 \ldots \ldots \ldots \ldots \ldots$

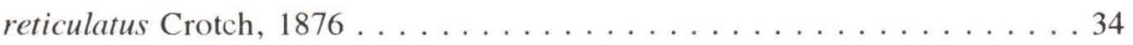

Revta bras. Zool. 11 (1): 1 - 175, 1994 
rhodosomus Lac., $1842 \ldots \ldots \ldots . \ldots \ldots$. . . . . . . . . . . . . 34

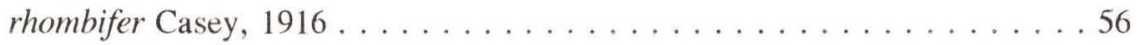

rhomboidalis Guérin, $1956 \ldots \ldots \ldots \ldots \ldots \ldots \ldots \ldots \ldots \ldots \ldots \ldots \ldots \ldots$

Rhynchothonius Crotch, $1876 \ldots \ldots \ldots \ldots \ldots 114$

Romodacne Delk., $1937 \ldots \ldots \ldots \ldots \ldots \ldots$

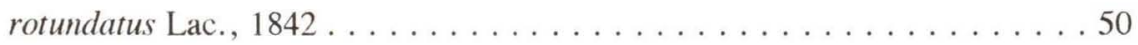

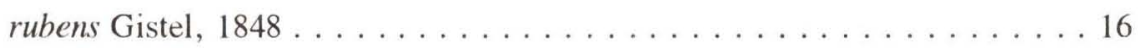

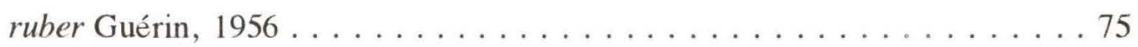

rubidus Dup., $1825 \ldots \ldots \ldots \ldots \ldots \ldots \ldots \ldots \ldots \ldots \ldots$

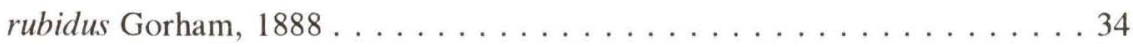

rubricatus Herbst, $1799 \ldots \ldots \ldots \ldots \ldots \ldots$

rubripennis Lac., $1842 \ldots \ldots \ldots \ldots \ldots \ldots \ldots \ldots$

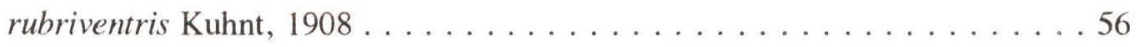

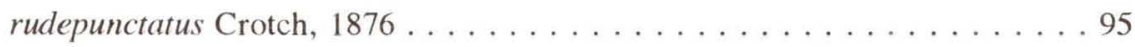

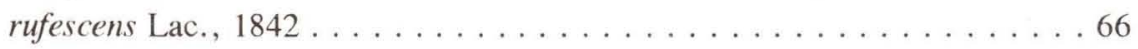

ruficeps Guér.-Mén., $1841 \ldots \ldots \ldots \ldots \ldots \ldots \ldots$

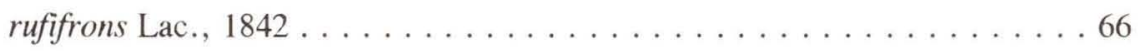

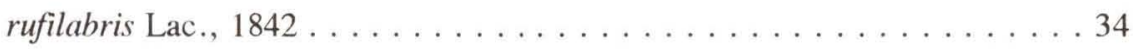

rufinus Lac., $1842 \ldots \ldots \ldots \ldots \ldots \ldots$. . . . . . . . . . . . . 43

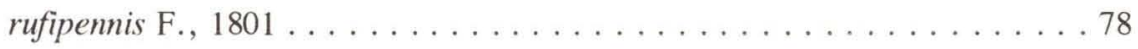

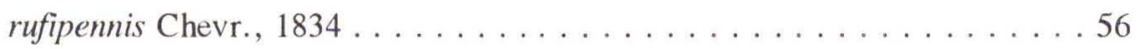

rufipennis Guérin, $1956 \ldots \ldots \ldots \ldots \ldots \ldots \ldots$

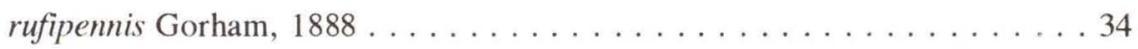

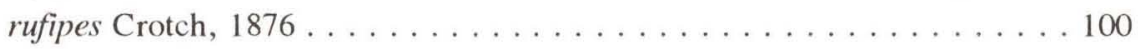

rufolimbatus Crotch, $1876 \ldots \ldots \ldots \ldots \ldots$

rufonotatus Crotch, $1876 \ldots \ldots \ldots$. . . . . . . . . . . . 69

rugipunctatus Crotch, $1876 \ldots \ldots \ldots \ldots 9 . \ldots \ldots \ldots$

rugithorax Crotch, $1876 \ldots \ldots \ldots \ldots \ldots \ldots \ldots \ldots \ldots$

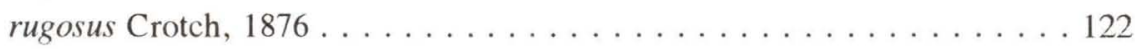

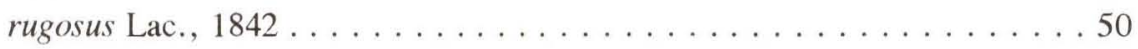

Saccomorphus Chevr., $1837 \ldots \ldots \ldots \ldots \ldots \ldots \ldots$

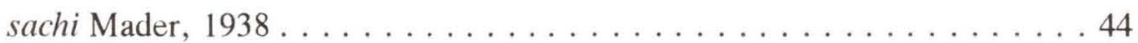

salamandra Erichson, $1847 \ldots \ldots \ldots \ldots \ldots \ldots \ldots \ldots \ldots \ldots \ldots \ldots$

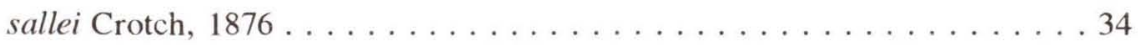

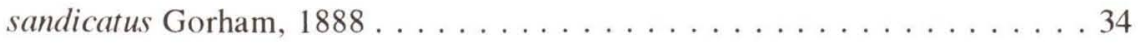

sanguinans Guérin, $1948 \ldots \ldots \ldots \ldots \ldots \ldots \ldots \ldots \ldots$ 


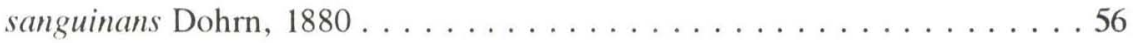

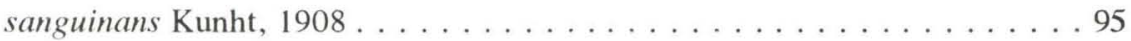

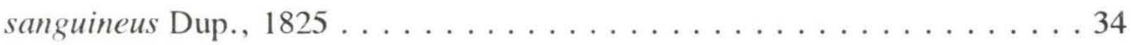

sanguiniceps Kuhnt, $1908 \ldots \ldots \ldots \ldots \ldots$

sanguinicollis Kuhnt, $1910 \ldots \ldots \ldots \ldots \ldots$. . . . . . . . . 108

sanguinolentus Lac., $1842 \ldots \ldots \ldots \ldots \ldots \ldots \ldots \ldots$

sanguinosus Crotch, $1876 \ldots \ldots \ldots \ldots \ldots \ldots \ldots$

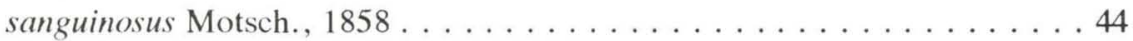

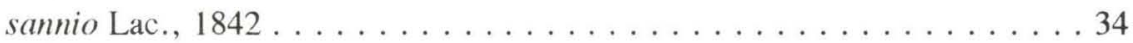

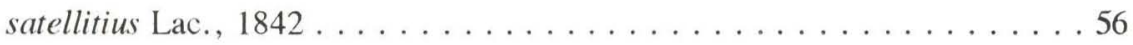

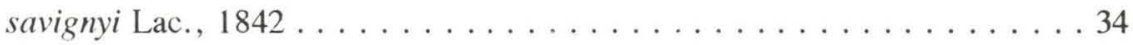

Scaeother Gorham, $1888 \ldots \ldots \ldots$. . . . . . . . . . . . 39

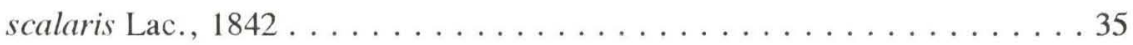

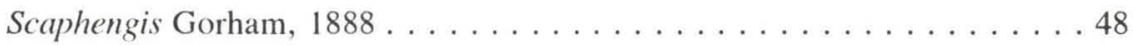

scaphidomorphoides Crotch, $1876 \ldots \ldots \ldots \ldots$. . . . . . . . . 95

Scaphidomorphus Hope, 1841 . . . . . . . . . . . . . . . . . . 110

scaphinotus Lac., $1842 \ldots \ldots \ldots \ldots \ldots \ldots \ldots \ldots \ldots \ldots \ldots$

scenicus Erichson, $1847 \ldots \ldots \ldots \ldots \ldots \ldots$

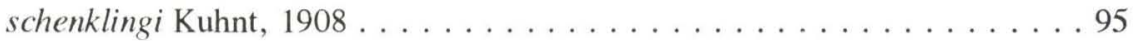

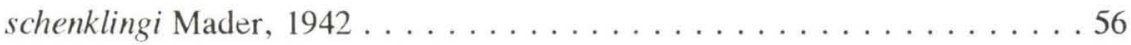

scita Curran, $1944 \ldots \ldots \ldots \ldots \ldots$. . . . . . . . . . . . . . . 100

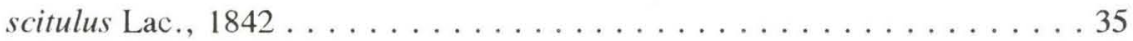

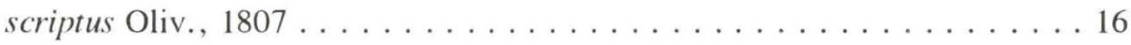

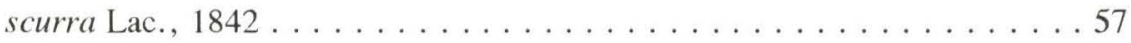

scutellaris Gorham, $1888 \ldots \ldots \ldots \ldots \ldots \ldots \ldots \ldots \ldots \ldots \ldots$

scutellaris Lac., $1842 \ldots \ldots \ldots \ldots \ldots \ldots \ldots \ldots \ldots \ldots$

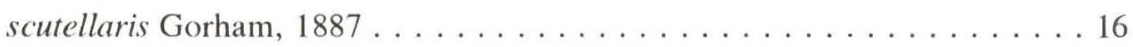

scutellata Kuhnt, $1908 \ldots \ldots \ldots \ldots \ldots$

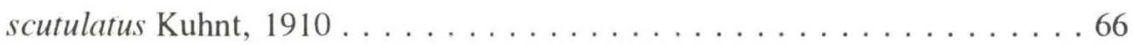

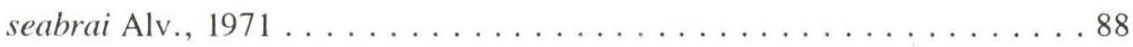

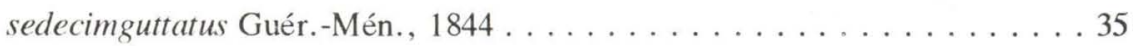

sedecimguttatus Oliv., $1792 \ldots \ldots \ldots \ldots \ldots \ldots$

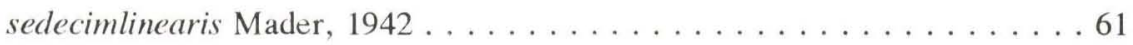

sedecimmaculatus Buquet, $1840 \ldots \ldots \ldots \ldots \ldots \ldots \ldots$

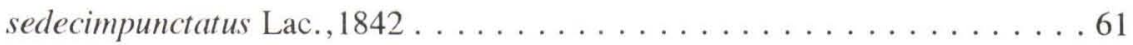

sedecimpustulatus Lac., $1842 \ldots \ldots \ldots \ldots \ldots \ldots \ldots \ldots$ 
sedecimstrigatus Mader, 1942 ....................61

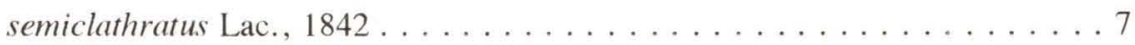

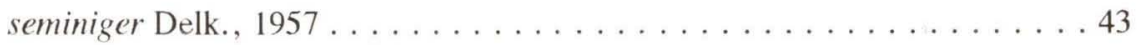

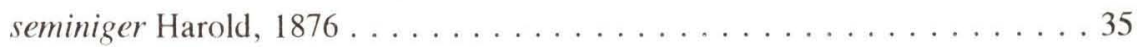

seminulus Lac., $1842 \ldots \ldots \ldots$. . . . . . . . . . . . . . . . . 43

semipunctatus Germ., $1824 \ldots \ldots \ldots \ldots \ldots \ldots \ldots \ldots$

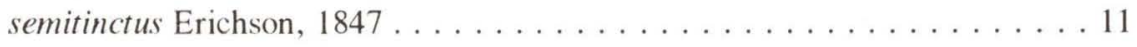

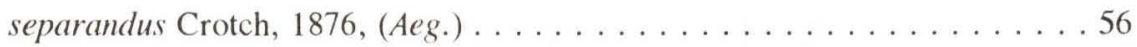

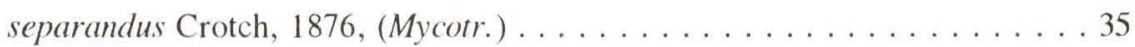

septemmaculatus Guérin, $1956 \ldots \ldots \ldots \ldots$

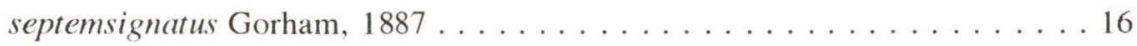

serenus Delk., $1957 \ldots \ldots \ldots$. . . . . . . . . . . . . . . . . . . . . . . .

seriatus Kuhnt, 1908 . . . . . . . . . . . . . . . . 105

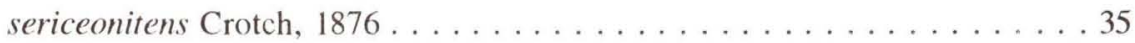

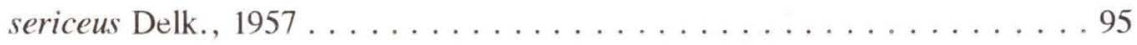

serripennis Guér.-Mén., $1841 \ldots \ldots \ldots \ldots$. . . . . . . . . 116

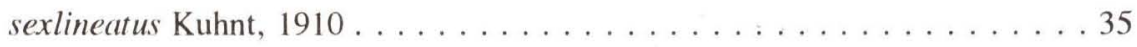

sexmaculatus Crotch, $1876 \ldots \ldots \ldots \ldots \ldots \ldots \ldots$

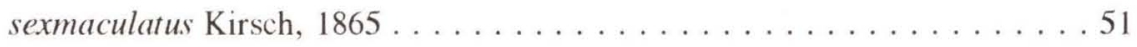

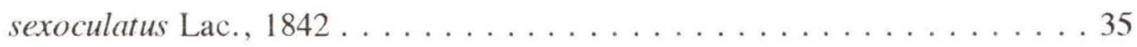

sexpunctatus Dup., $1825 \ldots \ldots \ldots \ldots \ldots \ldots \ldots \ldots \ldots$

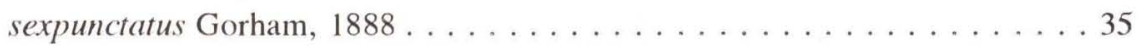

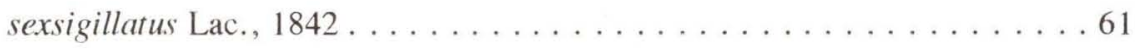

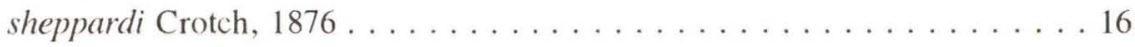

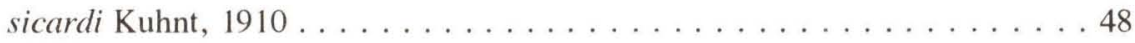

sicarius Lac., $1842 \ldots \ldots \ldots \ldots \ldots \ldots \ldots \ldots \ldots$

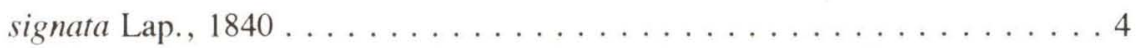

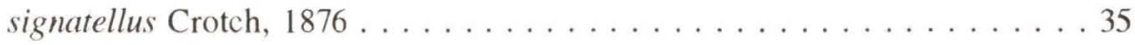

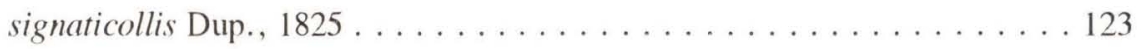

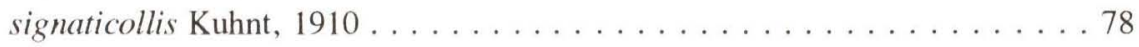

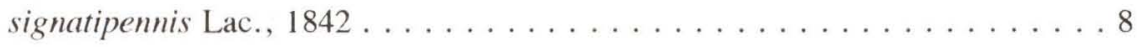

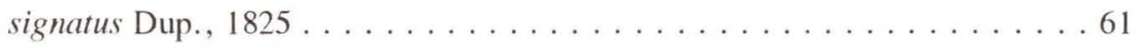

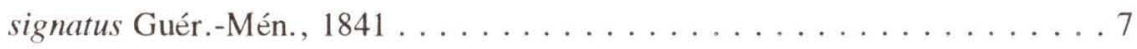

silaceus Lac., $1842 \ldots \ldots$. . . . . . . . . . . . . . . . . 36

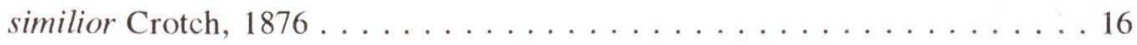

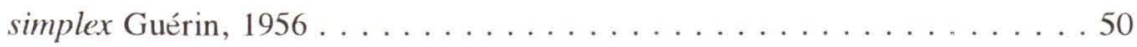


simplex Lac., $1842 \ldots \ldots \ldots \ldots \ldots \ldots \ldots \ldots \ldots \ldots \ldots \ldots$

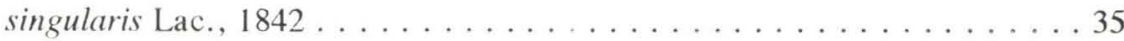

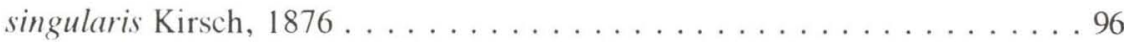

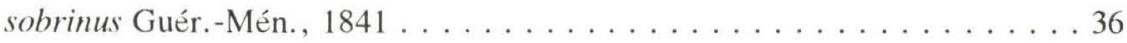

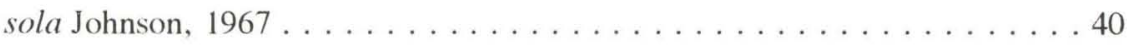

sordidus Gorham, $1898 \ldots \ldots \ldots \ldots \ldots \ldots \ldots \ldots \ldots$

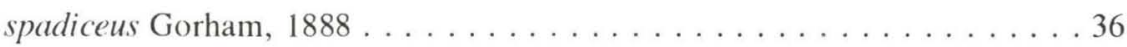

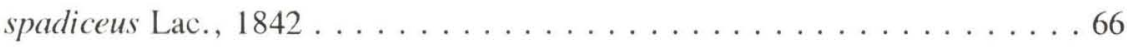

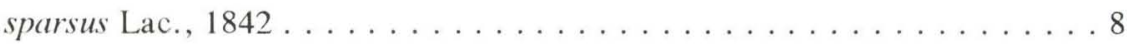

spectabilis Deelder, $1942 \ldots \ldots \ldots \ldots \ldots \ldots \ldots \ldots \ldots \ldots \ldots \ldots$

spectabilis Lac., $1842 \ldots \ldots \ldots \ldots \ldots \ldots \ldots \ldots \ldots \ldots \ldots \ldots \ldots \ldots$

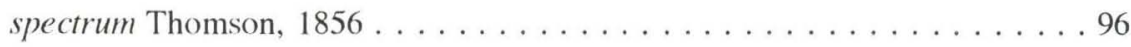

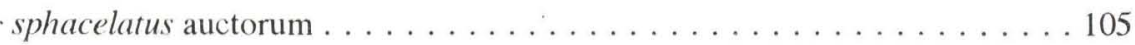

Sphenoxus Lac., $1842 \ldots \ldots \ldots \ldots$. . . . . . . . . . . . . 87

spilotus Gorham, $1888 \ldots \ldots \ldots \ldots \ldots \ldots \ldots \ldots \ldots \ldots$

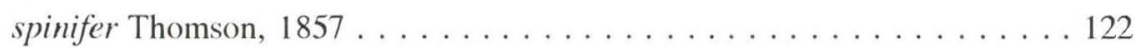

spinolae Guér.-Mén., 1844 . . . . . . . . . . . . . . . . . . . . . . . 117

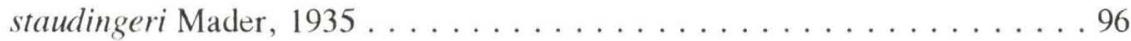

stellio Erichson, $1847 \ldots \ldots \ldots \ldots \ldots \ldots \ldots \ldots \ldots \ldots$

Sternolobus Guér.-Mén., $1841 \ldots \ldots \ldots \ldots$

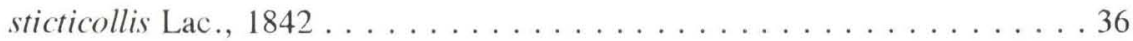

sticticus Erichson, $1847 \ldots \ldots \ldots \ldots \ldots$

stillatus Gorham, $1888 \ldots \ldots \ldots \ldots \ldots \ldots \ldots \ldots$

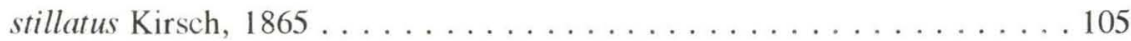

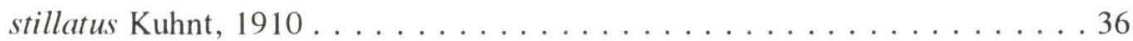

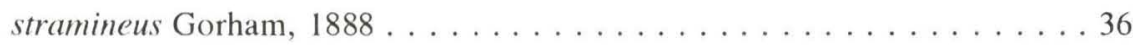

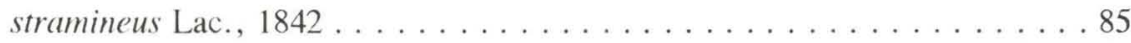

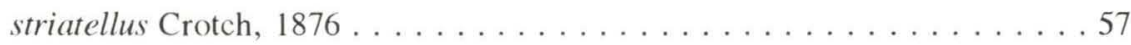

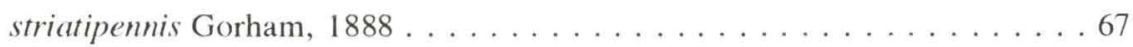

strigicollis Gorham, $1888 \ldots \ldots \ldots \ldots \ldots \ldots \ldots$

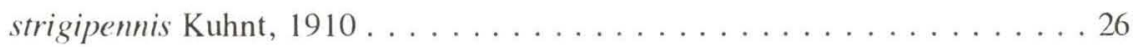

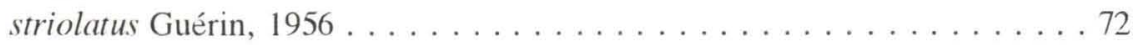

Strongylosomus Chevr, $1837 \ldots \ldots \ldots \ldots \ldots \ldots \ldots$

subcylindricus Lac., $1842 \ldots \ldots \ldots \ldots \ldots \ldots$

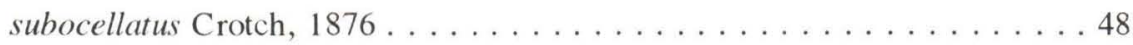

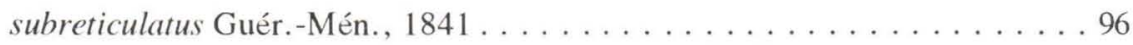


subsanguineus Crotch, $1876 \ldots \ldots \ldots \ldots \ldots$

subsignatus Lac., $1842 \ldots \ldots \ldots \ldots$. . . . . . . . . . . . . . . . 67

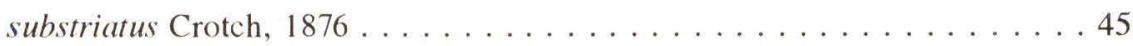

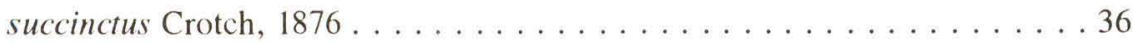

sulphurifer Lac., $1842 \ldots \ldots \ldots \ldots \ldots$. . . . . . . . . . . . . . 67

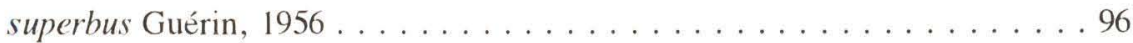

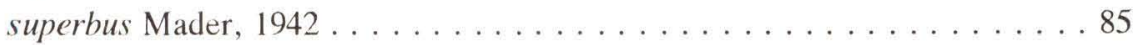

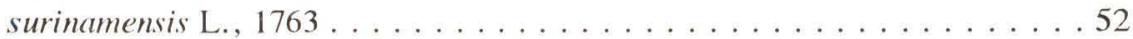

surinamensis Voet, $1778 \ldots \ldots \ldots \ldots 7 \ldots \ldots \ldots \ldots$

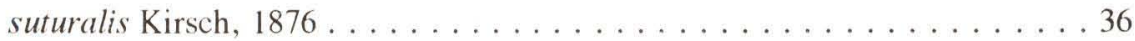

suturalis Lac., $1842 \ldots \ldots \ldots \ldots \ldots \ldots \ldots \ldots \ldots \ldots$

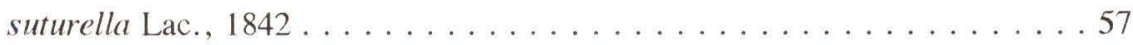

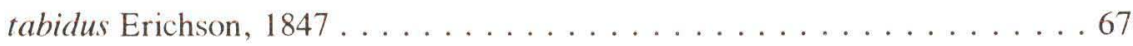

taeniatus Mader, $1942 \ldots \ldots \ldots \ldots \ldots$. . . . . . . . . . . 83

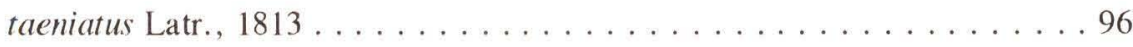

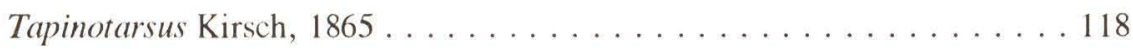

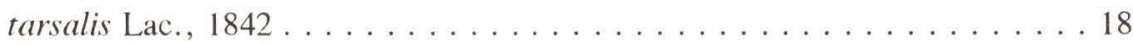

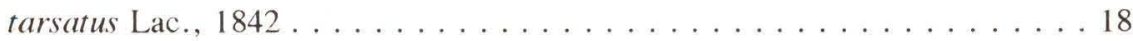

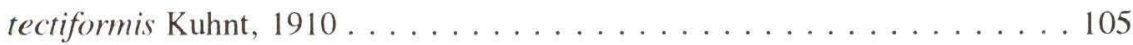

tenuecinctus Lac., $1842 \ldots \ldots \ldots \ldots \ldots$. . . . . . . . . . . . 72

tenuecinctus Kuhnt, $1908 \ldots \ldots \ldots \ldots \ldots 2$

teresae Alv., 1976 . . . . . . . . . . . . . . . . . . . . . . . . . . . . 100

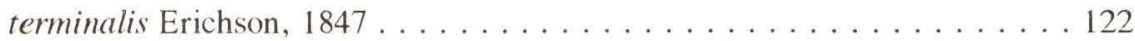

terminalis Kuhnt, $1908 \ldots \ldots \ldots \ldots \ldots \ldots$

terminalis Lac., $1842 \ldots \ldots \ldots \ldots$. . . . . . . . . . . . . . . . . 32

ternotatus Gorham, $1888 \ldots \ldots \ldots \ldots \ldots \ldots \ldots$

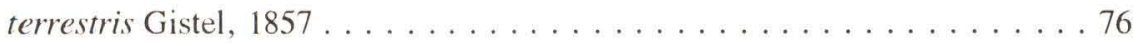

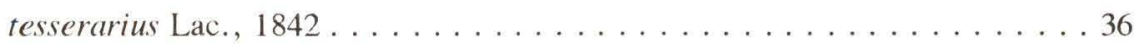

testaceipennis Mader, $1942 \ldots \ldots \ldots \ldots \ldots \ldots \ldots$

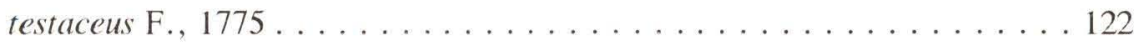

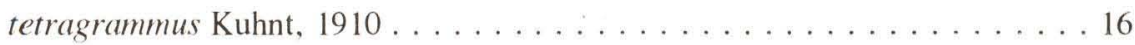

tetraspilotus Guér.-Mén., $1844 \ldots \ldots \ldots \ldots \ldots \ldots$

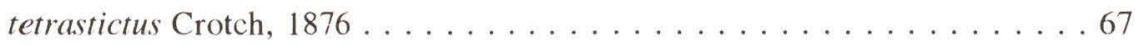

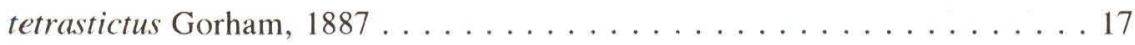

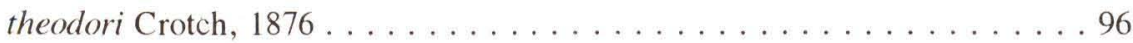

Thonius Lac., $1842 \ldots \ldots \ldots \ldots$. . . . . . . . . . . . . . . . . . 45 


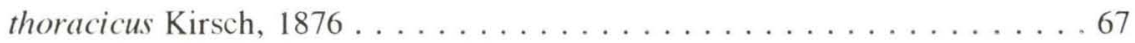

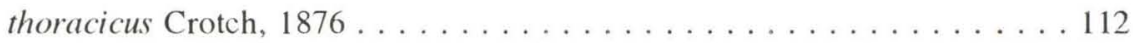

thoracicus Gistel, 1848 . . . . . . . . . . . . . . . . . . . . . 109

thoracicus Kuhnt, $1908 \ldots \ldots \ldots \ldots \ldots \ldots$

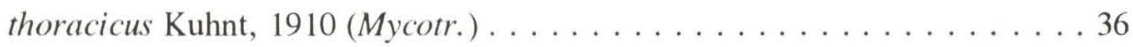

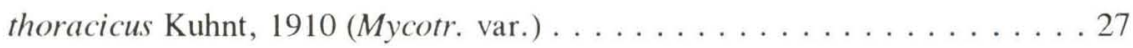

thoracicus Oliv., $1807 \ldots \ldots \ldots \ldots$. . . . . . . . . . . . 46

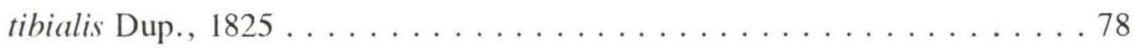

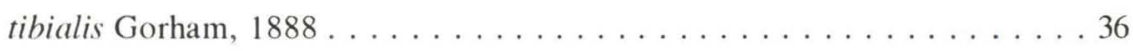

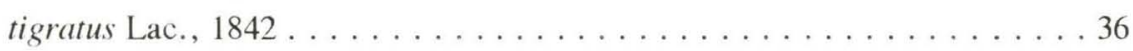

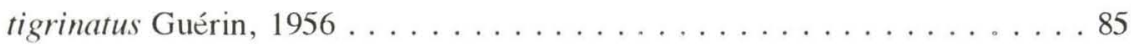

tigrinipennis Lac., $1842 \ldots \ldots \ldots \ldots \ldots \ldots \ldots \ldots \ldots \ldots \ldots$

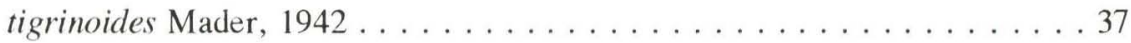

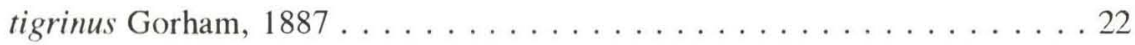

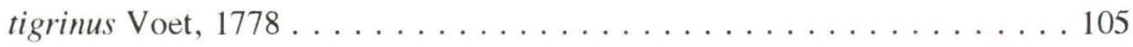

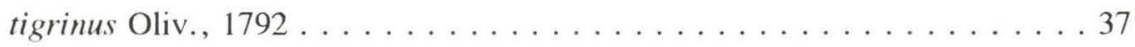

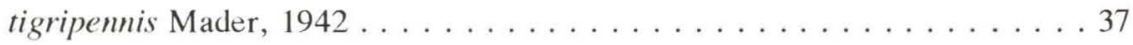

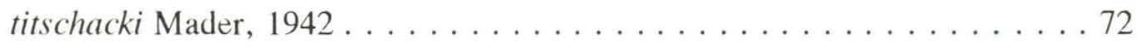

torquatus Lac., $1842 \ldots \ldots \ldots \ldots \ldots \ldots \ldots \ldots \ldots \ldots \ldots$

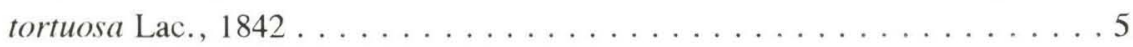

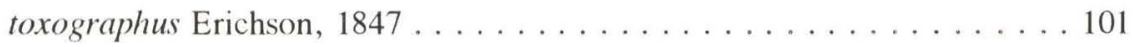

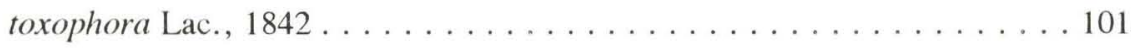

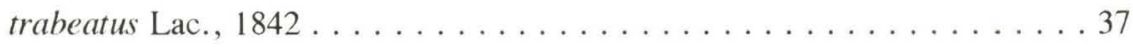

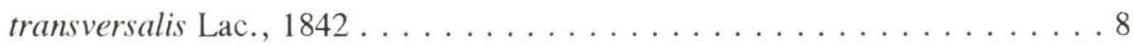

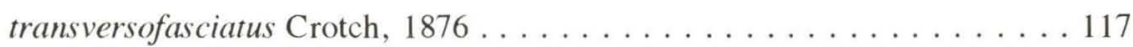

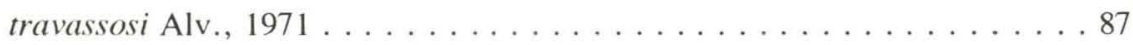

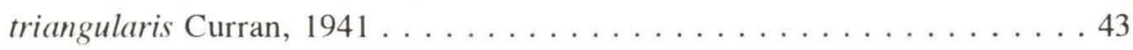

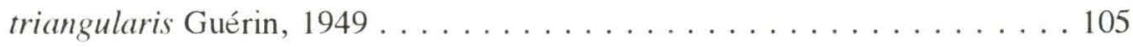

trichromaticus Alv., $1976 \ldots \ldots \ldots \ldots \ldots \ldots$

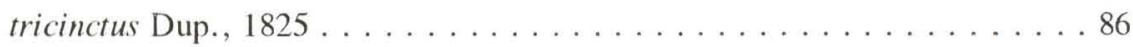

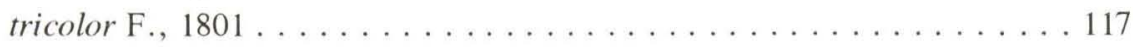

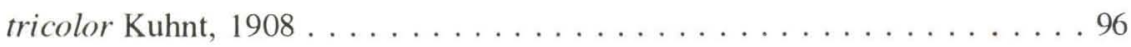

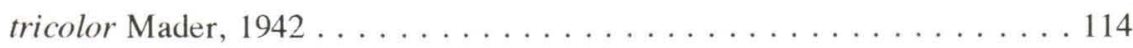

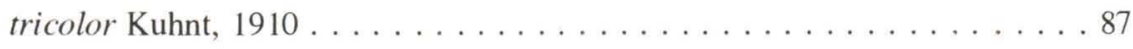

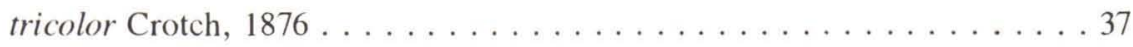

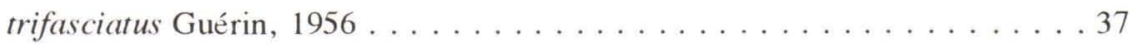

Revta bras. Zool. 11 (1): 1 - 175, 1994 


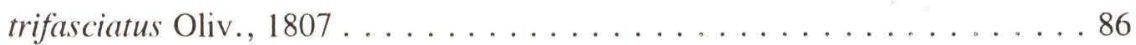

trifasciatus Lac., $1842 \ldots \ldots \ldots \ldots \ldots \ldots \ldots \ldots$

trilineatus Kuhnt, $1908 \ldots \ldots \ldots \ldots \ldots 7 \ldots \ldots \ldots \ldots$

trimaculatus Kirsch, 1865 . . . . . . . . . . . . . . . . . . 122

tripartitus Guérin, $1949 \ldots \ldots \ldots \ldots$

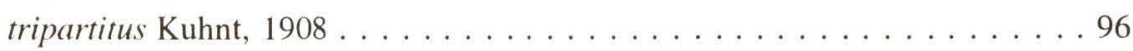

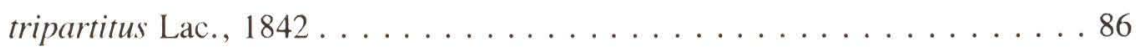

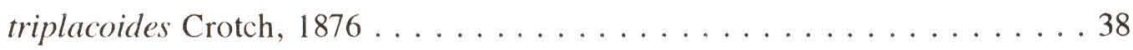

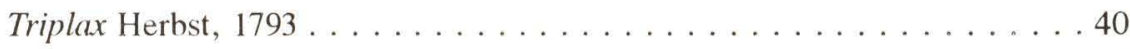

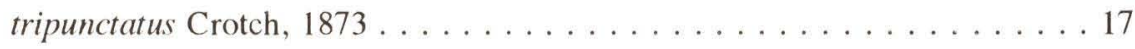

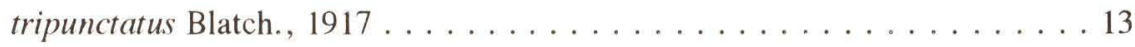

tripunctatus Guérin, $1946 \ldots \ldots \ldots \ldots \ldots \ldots \ldots$

tripunctatus Dup., $1825 \ldots \ldots \ldots \ldots \ldots \ldots \ldots \ldots$

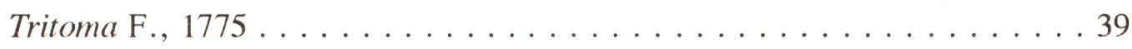

Tritomapara Alv., $1970 \ldots \ldots \ldots \ldots \ldots$. . . . . . . . . . 38

trizonatus Germ., $1924 \ldots \ldots$. . . . . . . . . . . . . . . . . . 109

truncatus Lac., $1842 \ldots \ldots \ldots \ldots \ldots \ldots \ldots \ldots \ldots$

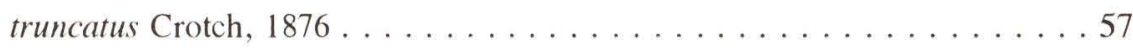

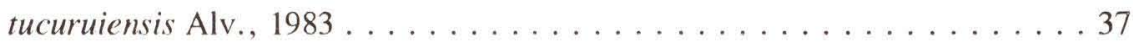

tumidus Lac., $1842 \ldots \ldots \ldots \ldots \ldots \ldots \ldots \ldots \ldots$

Typocephalus Chevr., $1837 \ldots \ldots \ldots \ldots$. . . . . . . . . 87

ucayalensis Gorham, $1889 \ldots \ldots \ldots \ldots \ldots \ldots \ldots$

umbonatus Lac., $1842 \ldots \ldots \ldots \ldots$. . . . . . . . . . . . . . . 122

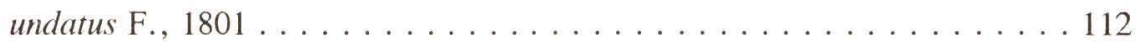

undatus Oliv., $1792 \ldots \ldots \ldots \ldots \ldots \ldots \ldots \ldots \ldots \ldots$

undulatus Gorham, $1887 \ldots \ldots \ldots \ldots \ldots \ldots \ldots$

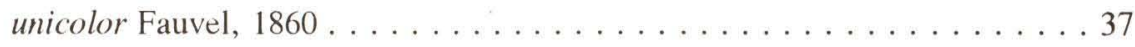

unicolor Guér.-Mén., $1844 \ldots \ldots \ldots \ldots$. . . . . . . . . . 46

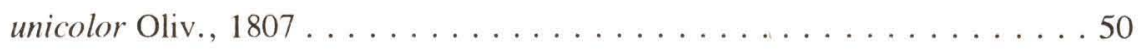

unicoloripennis Mader, $1942 \ldots \ldots \ldots \ldots \ldots \ldots$

unifasciata Schenkl., $1919 \ldots \ldots \ldots \ldots \ldots \ldots \ldots \ldots$

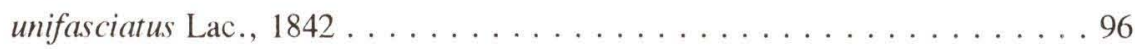

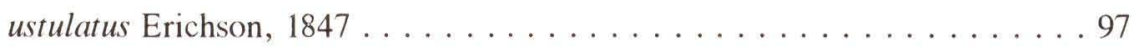

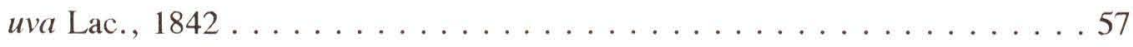

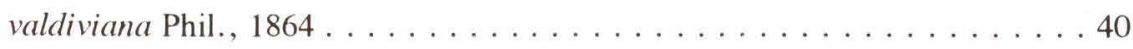

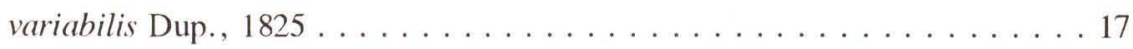


varians Crotch, $1876 \ldots \ldots \ldots \ldots$. . . . . . . . . . . . 97

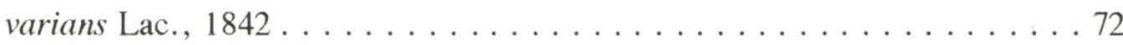

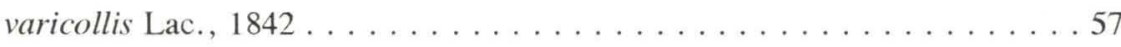

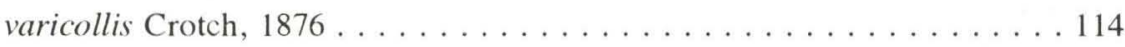

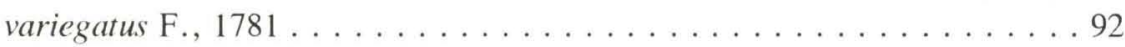

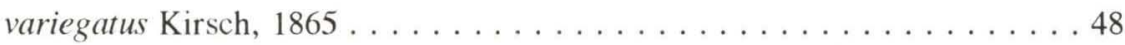

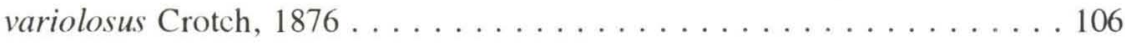

variomaculatus Kuhnt, $1908 \ldots \ldots \ldots \ldots \ldots$. . . . . . . . . 97

varius Gmelin, $1790 \ldots \ldots \ldots \ldots \ldots$. . . . . . . . . . . . 92

velatus Lac., $1842 \ldots \ldots \ldots \ldots \ldots \ldots \ldots$

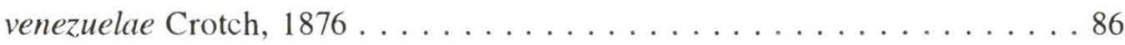

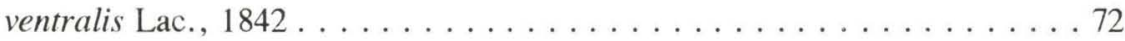

venustus Lac., $1842 \ldots \ldots \ldots$. . . . . . . . . . . . . . . . . . . 19

vernicatus Gorham, $1888 \ldots \ldots \ldots \ldots \ldots \ldots \ldots \ldots \ldots \ldots \ldots$

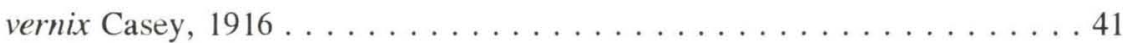

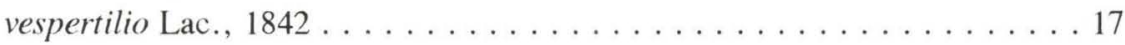

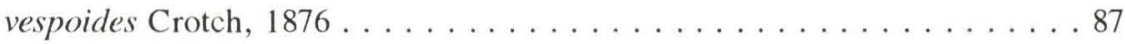

vetula Lac., $1842 \ldots \ldots \ldots \ldots \ldots \ldots$. . . . . . . . . . . . 67

vicinus Guér.-Mén., $1841 \ldots \ldots \ldots \ldots 6$

vigintiguttatus Dup., $1825 \ldots \ldots \ldots \ldots \ldots \ldots \ldots \ldots \ldots \ldots \ldots \ldots$

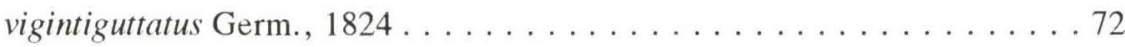

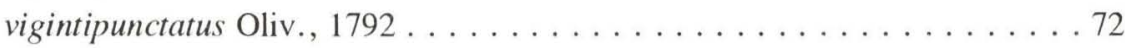

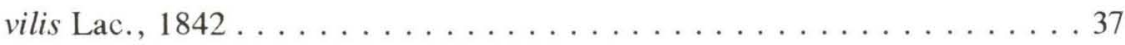

vinculatus Lac., $1842 \ldots \ldots \ldots \ldots \ldots$. . . . . . . . . . . . . 97

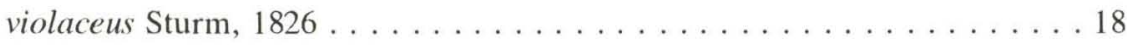

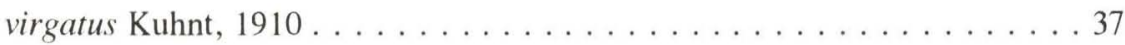

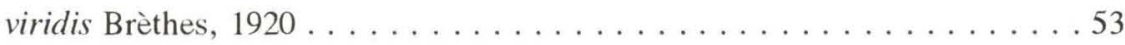

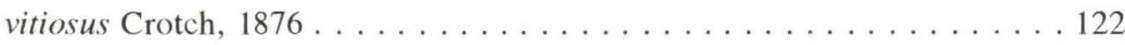

vittatus Crotch, $1876 \ldots \ldots \ldots \ldots \ldots \ldots$

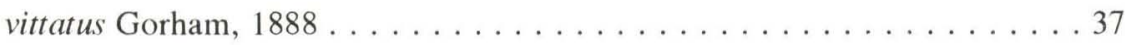

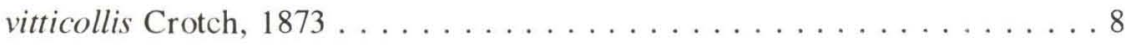

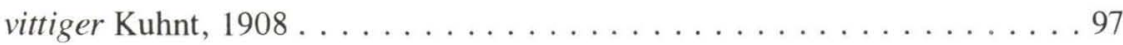

vivida Gorham, $1888 \ldots \ldots \ldots \ldots \ldots \ldots \ldots \ldots \ldots \ldots \ldots$

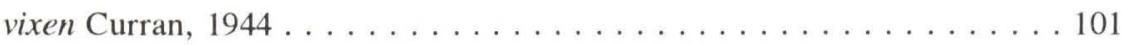

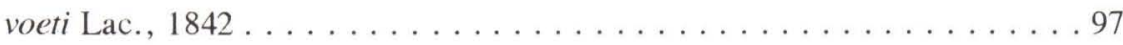

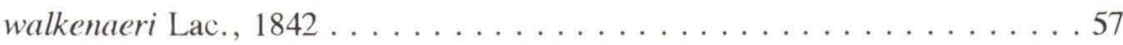


wehrlei Boyle, $1954 \ldots \ldots \ldots \ldots \ldots \ldots \ldots \ldots \ldots \ldots \ldots \ldots \ldots$

westwoodi Guér.-Mén., $1841 \ldots \ldots \ldots \ldots \ldots \ldots \ldots \ldots \ldots \ldots \ldots$

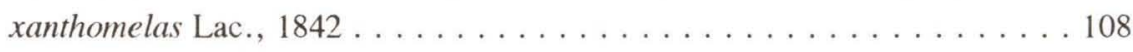

xanthomelas Crotch, $1873 \ldots \ldots \ldots \ldots \ldots \ldots \ldots \ldots$

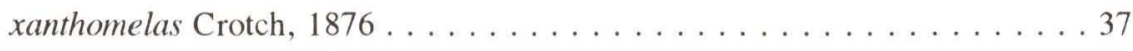

xanthosomus Lac., 1842 . . . . . . . . . . . . . . . . . . . 29

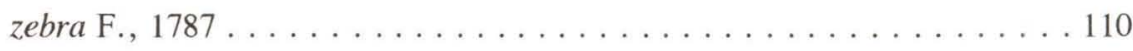

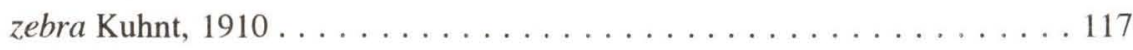

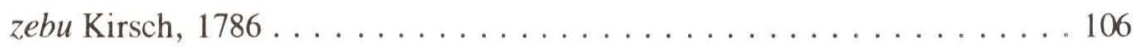

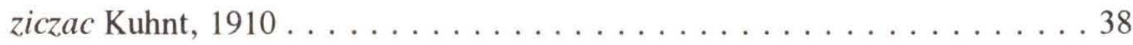

ziczac Tasch., $1870 \ldots \ldots \ldots$. . . . . . . . . . . . . . . . . . .97

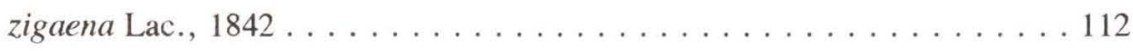

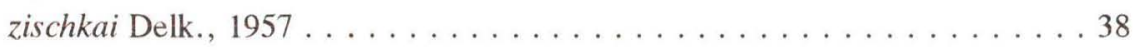

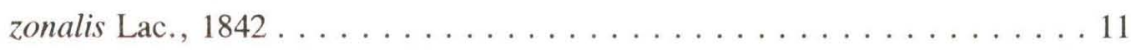

Zonarius Hope, $1841 \ldots \ldots \ldots$. . . . . . . . . . . . . . . . 107

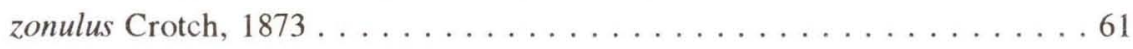

Recebido em 23.XII.1991; aceito em 04.IV.1994. 